\title{
Metabolic Signals in SySTEMic ACQUiRed RESISTANCE
}

\author{
Dissertation \\ for the award of the degree \\ "Doctor rerum naturalium" \\ of the University of Goettingen \\ within the doctoral program \\ "Plant Response To Eliminate Critical Threats" \\ of the Georg-August-University School of Science (GAUSS) \\ submitted by \\ Dmitrij Aleksandrovic Rekhter \\ from Charkow, Ukraine
}

March 2018

Department of Plant biochemistry

Albrecht-von-Haller-Institute for Plant Sciences

Georg-August University Goettingen 


\section{Thesis committee}

Prof. Dr. Ivo Feussner, Department of Plant Biochemistry, Albrecht-von-Haller-Institute for Plant Science, University of Goettingen

Prof. Dr. Christiane Gatz, Department of Plant Molecular Biology and Physiology, AlbrechtvonHaller-Institute for Plant Science, University of Goettingen

Prof. Dr. Yuelin Zhang, Department of Botany, University of British Columbia, Vancouver, Canada

\section{Members of the Examination Board}

Referee: Prof. Dr. Ivo Feussner, Department of Plant Biochemistry, Albrecht-von-Haller-Institute for Plant Science, University of Goettingen

$2^{\text {nd }}$ Referee: Prof. Dr. Christiane Gatz, Department of Plant Molecular Biology and Physiology, Albrecht-von-Haller-Institute for Plant Science, University of Goettingen

\section{Further members of the Examination Board}

Prof. Dr. Yuelin Zhang, Department of Botany, University of British Columbia, Vancouver, Canada

Prof. Dr. Andrea Polle, Department for Forest Botany and Tree Physiology, Buesgen-Institute, University of Goettingen

Prof. Dr. Volker Lipka, Department of Plant Cell Biology, Albrecht-von-Haller Institute for Plant Sciences, University of Goettingen

PD Dr. Marcel Wiermer, RG Molecular Biology of Plant-Microbe Interactions, Albrecht-vonHaller-Institute for Plant Sciences, University of Goettingen 
"Never neglect an extraordinary appearance or happening. It may be usually is, in fact - a false alarm that leads to nothing, but may on the other hand be the clue provided by fate to lead you to some important advance."

- Sir Alexander Fleming 


\section{Affidavit}

Hereby, I confirm that the dissertation "Metabolic Signals in Systemic Acquired Resistance" has been written independently and no other sources and aids were used than quoted.

Dmitrij Rekhter

Goettingen 2019 


\section{Table of Contents}

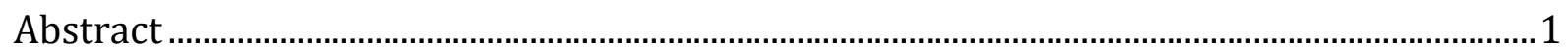

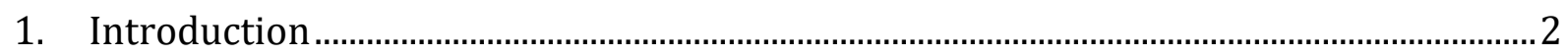

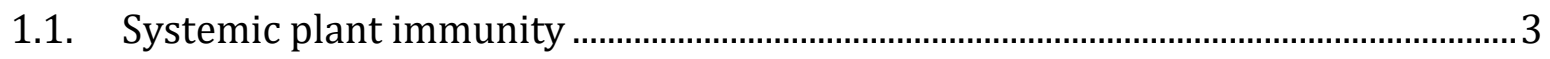

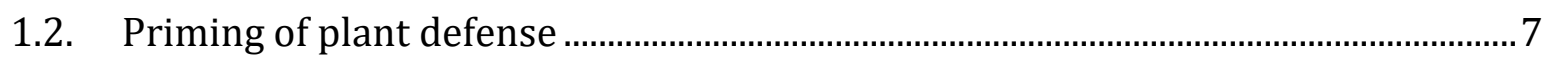

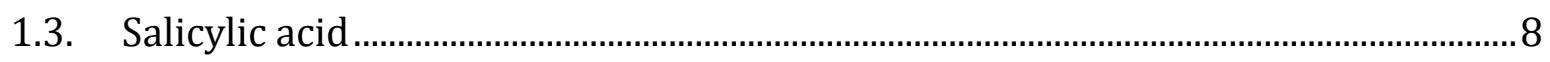

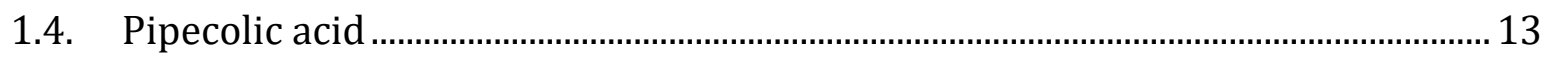

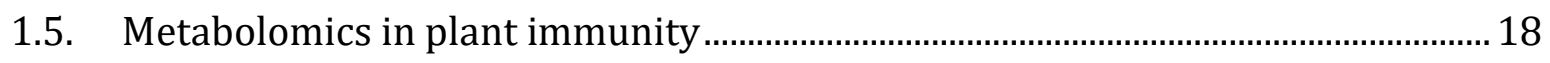

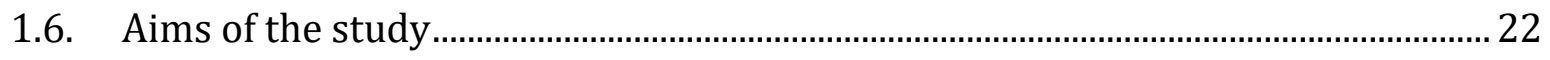

2. Article I. Characterization of a Pipecolic Acid Biosynthesis Pathway Required for

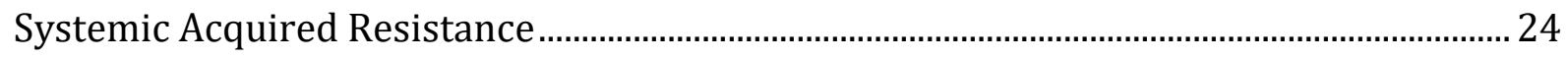

3. Article II. From isochorismate to salicylate: a new reaction mechanism for salicylic acid biosynthesis .55

4. Article III. Is Enhanced Disease Susceptibility 5 the Achilles' Heel of Plant Immunity?

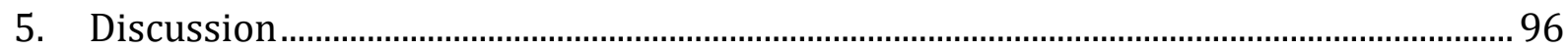

5.1. Pip biosynthesis in systemic leaves depends on SARD4 …..................................... 97

5.2. PBS3 is the yet missing link in the SA biosynthesis...............................................100

5.3. EDS5 - gatekeeper in SA and NHP biosynthesis alike ............................................107

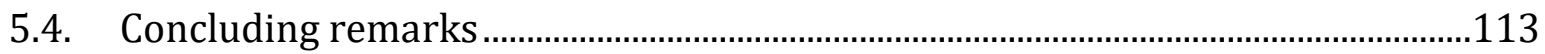

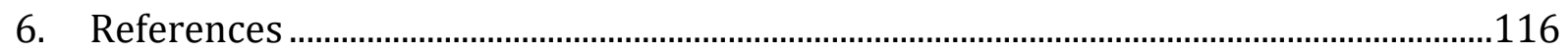

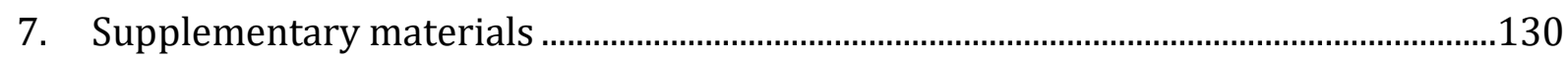

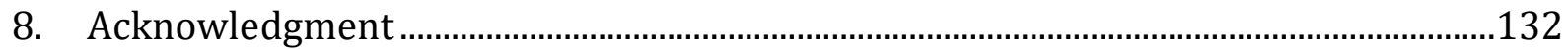

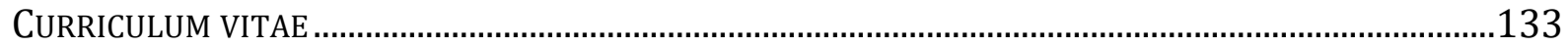




\section{Abstract}

Plants are sessile organisms and therefore, they cannot escape from potential threats. Moreover, plants do not possess specialized mobile immune cells, which would maintain the immune system, as we know from mammals. In order to cope with pathogen attackers, plants developed a complex and multilayered defense system (Fu and Dong, 2013; Bacete et al., 2018). To coordinate the immune response, plants produce a number of signaling compounds. These metabolites regulate on the one hand the processes in infected tissues and spread on the other hand through the plant to alarm distal organs (Fu and Dong, 2013; van Loon, 2016). The exact spatiotemporal organized biosynthesis of these signaling compounds is crucial for the establishment of an efficient defense against the corresponding pathogens, without wasting important resources. Against biotrophic pathogen, salicylic acid (SA) and pipecolic acid (Pip) respectively $N$-hydroxy pipecolic acid (NHP) are the most important signaling compounds. Although the importance of these small metabolites in plants has been known for decades, parts of their biosynthesis stayed elusive so far (Hartmann and Zeier, 2018; Klessig et al., 2018). This motivated the work presented here, to study metabolic pathways that are responsible for the biosynthesis of these signaling compounds.

Utilizing immune deficient mutant lines of the model organism Arabidopsis thaliana, untargeted metabolite fingerprint analysis was performed in order to identify yet missing links in the biosynthesis pathways of SA and Pip/NHP. Subsequent in vitro protein assays enabled the identification of systemic acquired resistance-deficient 4 (SARD4) as the yet missing ketimine reductase in the pathogen induced biosynthesis of Pip (Article I) in systemic leaves. In case of SA biosynthesis, it was shown previously that the formation of isochorismic acid (ISC) is crucial for the pathogen induced accumulation of SA (Garcion et al., 2008). However, the enzymatic step from ISC to SA remained elusive in plants. We found that avrPphB susceptible 3 (PBS3) catalyzes the conjugation of ISC with glutamic acid to yield ISC-9-glutamate (ISC-9-Glu). This compound decays non-enzymatically to give rise to SA (Article II).

Both, Pip and ISC, are synthesized in plastids, whereas their metabolism occurs in the cytosol (Dempsey et al., 2011). A plastidial exporter is therefore required to transport these compounds into the cytosol (Hartmann and Zeier, 2018). We gathered strong evidences that enhanced disease susceptibility 5 (EDS5) is responsible for the export of both, ISC and Pip (Article III). Together, these studies improved the understanding of the biosynthesis as well as the spatial distribution of the signaling compounds SA and Pip/NHP, which are key regulators of plant immunity. 


\section{Introduction}

Plants are exposed to constant environmental stresses, such as temperature changes or pathogen attacks. As sessile organisms, they could not escape and thus developed other strategies to cope with potential threats (Fu and Dong, 2013; van Loon, 2016). The first layer of protection is the cell wall. It is essential for the sensing of a stress as well as a physical barrier against invaders. Its physical and chemical properties prevent the majority of invasions (Bacete et al., 2018). As the second layer of plant defense, plasma membrane bound cell wall associated patter recognition receptors like flagellin sensing 2 (FLS2) or chitin elicitor receptor kinase 1 (CERK1) are monitoring the presence of pathogens, which could penetrate the rigid barrier (Boutrot and Zipfel, 2017). The detection of microbe associated molecular patterns (MAMPs) at the plant surface activates cellular signaling. This MAMP triggered immunity (MTI) facilitates the production of reactive-oxygen species (ROS), callose deposition at the penetration site and the transcription of pathogenesis related (PR) genes (Bigeard et al., 2015). Additionally, an increased accumulation of cellular messenger like nitric oxide (NO), calcium ions and cyclic guanosine monophosphate (cGMP) can be measured upon activation of MTI (Seybold et al., 2014). In literature, sometimes MTI is called pathogenassociated molecular pattern (PAMP)-triggered immunity (PTI, (Bigeard et al., 2015)). This is a less accurate term than MTI, as not only pathogens but also beneficial bacteria possess for instance flagella (Reimer-Michalski and Conrath, 2016).

The coexistence of plants and microbes leads to a continuous development of pathogenicity strategies for a successful invasion. The development of effector proteins, which can be secreted into host cells, enabled the pathogens to partially bypass the MTI (Jones and Dangl, 2006). On the other hand, intracellular plant resistance $(R)$ proteins can recognize these effectors. This process subsequently leads to an enhanced immune system response. $R$ proteins typically have nucleotide binding (NB) sites and leucine rich repeat (LRR) regions (Collier and Moffett, 2009; Khan et al., 2016). A direct interaction of an effector with an $R$ protein is summarized by the gen-for-gen concept whereas an indirect recognition of an effector corresponds to the guard theory (Reimer-Michalski and Conrath, 2016). The gene-for-gene concept proposes that for each pathogenicity protein in an attacking microbe, there is a corresponding resistance gene in a non-susceptible plant (Flor, 1971). However, this has become somewhat outdated since the information on mechanisms of plant immunity has grown. A more accurate representation for 
effector-triggered immunity (ETI) is the guard theory. A good example for this theory is RPM1-interacting protein 4 (RIN4). It is targeted by four known bacterial effectors. The $R$ proteins resistant to P. Syringae 2 (RPS2) and resistance to P. Syringae pv Maculicola 1 (RPM1) "guard" the homeostasis of RIN1. Modification of RIN1 by bacterial effectors are recognized by RPS2 or RPM1 (Khan et al., 2016), which as a result induce a signaling cascade, which frequently leads to a hypersensitive response (HR). This programmed cell death prevents biotrophic pathogens from infecting neighboring tissues (Coll et al., 2011). ETI as well as MTI use similar signaling pathways. Thereby, the ETI response is stronger, faster and yields a longer phase of protections due to a prolonged expression of defense genes (Tsuda and Katagiri, 2010). The plant immunity is often represented in the zigzag model showing a chronological interplay of ETI and MTI (Jones and Dangl, 2006). This model, however, is an oversimplification. For example, it does not include necrotrophic pathogens, which induce cell death in the host cells in order to survive. If the recognition of MAMPs from necrotrophic microbes would lead automatically to HR, the plant immunity would collapse (Cook et al., 2015). In order to cope with environmental threats, the plants developed a fine-meshed immune system, which consists of many different interconnected layers. Only the precise cooperation of receptors, $R$ proteins and signal compounds enables a proper plant immunity, which can effectively protect plants against a large variety of pathogens.

The plant response to an infection is not restricted to the affected area but leads much more to an immunization of the whole plant. Already in the beginning of the $20^{\text {th }}$ century, several groups observed that subsequent infections with the same pathogen induce little to no symptoms in the successive inoculations (Chester, 1933). Later it was shown that systemic acquired resistance (SAR) provides resistance against a broad-spectrum of pathogens. With SAR, plants are armed against subsequent pathogenic attacks (Ross, 1961). Fascinatingly, this acquired resilience is not limited to the affected plant itself but can be even passed to the descendants(Luna et al., 2012). The protective effects are thereby not limited to the infected species, but also induce resistance in other neighboring plants.

\subsection{Systemic plant immunity}

An outstanding property of SAR is that it is active against a wide range of pathogens, although the local response was activated by an individual attacker. The prerequisite for 
the immunization of naïve tissues is a mobile signal, which can be transferred from the affected site throughout the whole body (Kachroo and Robin, 2013). Distal tissues must recognize this signal in order to activate the primed state. It was previously shown that at least some of these mobile signals are transported via the phloem (Jenns and Kuc, 1979) while others are volatile (Yi et al., 2009). Several compounds were identified so far as being critical for the establishment of SAR (Figure 1 and Figure 2). Salicylic acid (SA) is known to be a key regulator of plant immunity (Malamy et al., 1990). It is synthesized at the site of infection as well as at distal tissues (Métraux et al., 1990). Whether or not the transport of SA is also required for the establishment of SAR is still a point of debate (Vernooij et al., 1994; Shulaev et al., 1995). Beside SA, it was shown that the synthesis of the non-proteinogenic amino acid pipecolic acid (Pip) is also required for the

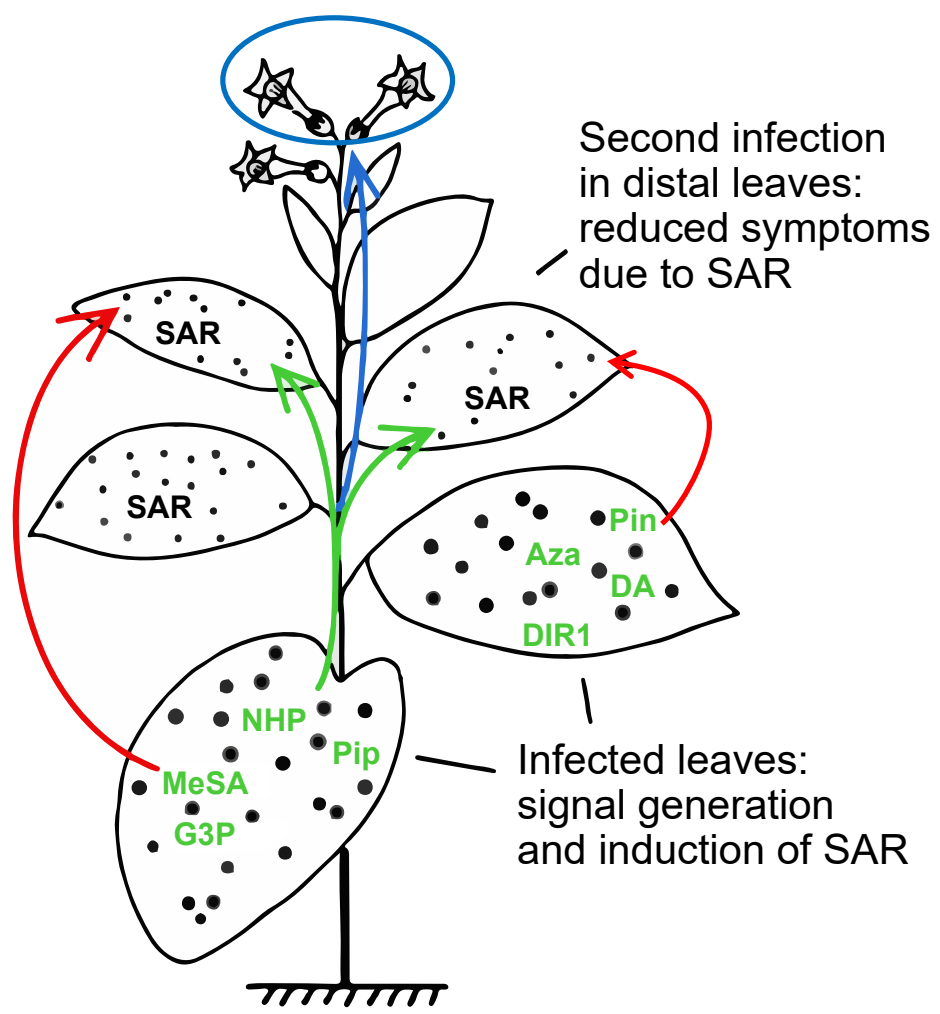

Figure 1. Establishment of systemic acquired resistance (SAR).

Upon infection, plants produce diverse chemical molecules in order to induce the local immune system. Dark dots in the infected leaves symbolize strong pathogenic symptoms. Some of these molecules can be transferred to distal tissues, where they induce the establishment of SAR. SAR allows the naïve leaves to mount a quicker and more robust immune response against subsequent pathogen attacks. Smaller dark dots in systemic leaves symbolize weaker pathogenic symptoms. Mobile signals can either move though the phloem (green arrows), or be spread as volatiles (red arrows). Several reports showed that SAR can be passed on to the offspring (symbolized by blue arrow and circle). Signal molecules for SAR may be: methyl salicylate (MeSA), lipid transfer protein defective in induced resistance 1 (DIR1), dehydroabietinal (DA), pinenes (Pin), glycerol-3-phosphate (G3P), azelaic acid (AzA), pipecolic acid (Pip) and N-hydroxy pipecolic acid (NHP). Chemical structures of these molecules are depicted in Figure 2. Modified from Adam et al., 2018. 
establishment of SAR. Although Pip itself is a potent initiator of plant immunity (Navarova et al., 2012), its derivate $N$-hydroxy pipecolic acid (NHP) was shown to be an even better inducer (Chen et al., 2018). Recently, it was reported that another signaling compound of plant immunity depends on the accumulation of Pip, namely azalaic acid (Aza, (Wang et al., 2018)). Aza requires the protein defective in induced resistance 1 (DIR1) for the translocation into the systemic leaves. DIR1 is also required for the SAR inducing characteristics of the tricyclic diterpenoid dehydroabietinal (DA, (Chaturvedi et al., 2012)). The biosynthesis of Aza as well as its downstream signaling is not fully understood yet ( $\mathrm{Yu}$ et al., 2013). The current model suggests that upon pathogen treatment, ROS and NO are formed (Gilroy et al., 2016), which subsequently chemically oxidize fatty acids. This process could result in the release of Aza (Wendehenne et al., 2014). The accumulation of Aza was proposed to induce the synthesis of glycerol-3phosphate (G3P), which is subsequently transported to distal tissues where it induces the<smiles>O=C(O)c1ccccc1O</smiles>

salicylic acid<smiles>COC(=O)c1ccccc1O</smiles>

methyl salicylate<smiles>O=C(O)C1CCCCN1</smiles>

pipecolic acid<smiles>O=C(O)C1CCCCN1O</smiles>

$N$-hydroxypipecolic acid<smiles>O=C(O)CCCCCCCC(=O)O</smiles>

azalaic acid<smiles>O=P(O)(O)OCC(O)CO</smiles>

glycerol-3-phosphate<smiles>CC(C)c1ccc2c(c1)CCC1C(C)(C=O)CCCC21C</smiles>

dehydroabientinal<smiles>CC1=CCC2CC1C2(C)C</smiles><smiles>C=C1CC[C@H](C(C)(C)C)C(=C)C1=C</smiles>

pinene mixture

Figure 2. Compounds which are described to be either directly involved in the establishment of systemic acquired resistance (SAR) or can induce SAR.

Pinenes were shown to be more potent to induce SAR as a mixture than when used individually (Riedlmeier et al., 2017). To display the SAR inductive properties, azalaic acid (Aza), glycerol-3phosphate (G3P), and dehydroabiental (DA) require the functional protein defective in induced resistance 1 (DIR1) for their translocation. 
biosynthesis of Pip (Wang et al., 2018). It needs further studies to prove this model, as the in vitro synthesis experiments revealed ambiguous results (Wang et al., 2014). G3P, on the other hand, is an essential metabolite in the biosynthesis of glycerolipids. It is not surprising that a disruption in the biosynthesis of G3P leads to severe phenotypes (Chanda et al., 2011). It was also shown that volatile compounds can induce SAR within and between plants in addition to these soluble molecules. SA methyl ester (MeSA, (Park et al., 2007)) as well as monoterpenes (Riedlmeier et al., 2017) are emitted from leaves after infection (Figure 2).

How those signals are received, recognized and processed in the distal tissues, is not properly understood (Singh et al., 2017; Hartmann and Zeier, 2018). The SA carboxyl methyl transferase (BSMT1), which synthesizes MeSA from SA, was shown to be essential for the establishment of SAR in some but not in all infection studies (Liu et al., 2011). Thus, the function of MeSA in the establishment of SAR remains unclear so far. However, Tripathi and colleagues could show in tobacco that the MeSA-esterase (SABP2) is required for the induction of resistance to tobacco mosaic virus (Tripathi et al., 2010). This enzyme hydrolyzes MeSA back to SA. If the presence of SABP2 is indeed necessary for plant immunity, MeSA might be more important for the immune system as it has been discussed so far (Attaran et al., 2009). Jasmonoyl-isoleucine (JA-Ile) is another lipid derived signaling compound in plants in addition to Aza, which is normally associated with the plants response to wounding and defense against necrotrophic pathogens (Yan and Xie, 2015). Several groups proposed an antagonistic relation between SA and JA-Ile signaling (Spoel, 2003). Recent studies were able to present a more sophisticated model for this relationship (Mur et al., 2006). Truman and colleagues (Truman et al., 2007) could show that JA-Ile production and signaling is activated during the ETI in infected leaves as well as in distal tissues. The role of JA-Ile in systemic leaves is controversial, as JA-Ile deficient mutants are not impaired in their ability to establish SAR (Attaran et al., 2009). For the site of infection, however, JA-Ile could be an important regulator of the immune response. One aspect could be that the accumulation of this phytohormone prevents a secondary infestation of necrotrophic pathogens at necrotic HR cells (Liu et al., 2016). On the other hand, a recent image-based study showed that JA-Ile responsive genes are expressed in cells, which surround the necrotic lesions and are not directly localized in the HR area. The observation of a certain spatial distribution led to the conclusion that the surrounding cells might regulate the expansion of the HR (Betsuyaku et al., 2018). When metabolic changes are studied in order to characterize plant responses to pathogenic attacks, often 
the whole leaf is homogenized. Thereby, this spatial distribution gets lost. In order to fully understand a plant response on a cellular level in a given tissue, a spatially resolved metabolomics approach (Etalo et al., 2015) might support the so far only image-based results.

\subsection{Priming of plant defense}

In order to establish systemic immunity, distal organs need to react appropriately to the signals that are sent from attacked plant tissues. Upon recognition, signal transduction has to be activated in systemic leaves. This may lead to changes on DNA, protein and metabolite level (Reimer-Michalski and Conrath, 2016). The concept of priming represents an enhanced sensitivity and a stronger immune response of tissues that experienced a previous independent stress to a subsequent infection (Martinez-Medina et al., 2016). Although the precise mechanism of signal recognition is not understood yet, the consequences are well documented. Priming with the synthetic SA analogue benzo(1,2,3)-thiadiazole-7-carbothioic acid S-methyl ester (BTH) was shown to induce the accumulation of the pattern recognition receptors FLS2 and CERK1 (Tateda et al., 2014). It is conceivable that the elevated presence of these receptors is responsible for an accelerated MTI during secondary infections. Signals, recognized by those receptors at the plasma membrane, may be transmitted further via mitogen-activated protein kinase (MPK) cascades. Upon priming, the expression levels of MPK3 and MPK6 were shown to be elevated (Beckers et al., 2009). Interestingly, these proteins were not phosphorylated at their activation site. This suggests that the expression and translation of these proteins are induced by priming, whereas the final activation is only facilitated upon subsequent infection. A similar mode-of-action is known for the SA receptor non expressor of PR genes 1 (NPR1, (Wu et al., 2012)), which is present in its inactive, oligomeric form in the cytosol. Upon activation of the immune system, the intermolecular disulfide bonds of NPR1 oligomers are reduced and the monomers translocate into the nucleus, where they are required for the expression of defense related genes (Mou et al., 2003). At later timepoints, however, NPR1 was found in oligomeric form again (Tada et al., 2008). Consequently, primed tissues are armed to fight back subsequent pathogen infections, without a full activation of a costly resource and potentially self-harming immune system. The elevated expression level of genes that are associated with plant defense is a general feature of primed tissues. Central immunity regulators, like enhanced disease 
susceptibility 1 (EDS1) and phytoalexin deficient 4 (PAD4, (Wiermer et al., 2005)), as well as genes, which are required for the biosynthesis of defense related metabolites, are rapidly expressed upon priming (Gruner et al., 2013). The number of genes with an altered expression varies depending on the experimental set up. In the report from 2013, the Zeier group showed that 1921 genes were up-regulated in infected leaves 24 hours past infection (hpi). Out of these immunity related genes, 299 were up- and 19 down regulated $48 \mathrm{hpi}$ in systemic leaves (Gruner et al., 2013). In a similar approach, the same group showed later that more than 3400 genes were up- and nearly 3000 genes were down regulated systemically 48 hpi (Bernsdorff et al., 2016). Although the precise number of genes induced in immune defense is still to be determined, there appears to be a core set of genes, which are mandatory for the establishment of immunity, locally as well as systemically. These include genes encoding enzymes for the synthesis of the signaling metabolites SA and Pip. On the other hand, JA-Ile-related genes seem to be up-regulated only locally. Consequently, also metabolic pathways that rely on JA-Ile signaling, such as the biosynthesis of glucosinolates, are not induced in systemic tissues (Gruner et al., 2013). Interestingly, the biosynthesis of SA and Pip is also significantly lower in systemic tissues than in local leaves after infection (Bernsdorff et al., 2016). There appears to be a metabolic threshold, which needs to be reached in order to induce HR. In inoculated tissues, in which HR is required to prevent the attacker from spreading to other organs, this threshold is exceeded, and the programmed cell death is activated. It seems that priming achieves a status in naïve tissues, which falls short of reaching this line. A subsequent pathogenic attack, however, will overcome the threshold much quicker (Martinez-Medina et al., 2016). Fascinatingly, plants that were exposed to infections produce offspring that show an enhanced disease resistance. Luna and coworker (Luna et al., 2012) suggested that these effects are due to histone modifications. Since then, several reports confirmed that epigenetic changes could pass on to the next generation not only in Arabidopsis but also in bean (Ramirez-Carrasco et al., 2017) and potato (Meller et al., 2018). Thereby, SA responsive genes again seem to be the key players in the establishment of a more robust immune response.

\subsection{Salicylic acid}

The pain relieving effect of plants that contain high amounts of SA has been known to mankind for thousands of years. A recent report found evidence that already the 
Neanderthals chewed poplar bark as self-medication (Weyrich et al., 2017). The first known extraction of the active compound from willow bark was reported from Buchner in the beginning of the $19^{\text {th }}$ century (Buchner, 1828), before Kolbe and colleagues developed a chemical method for the synthesis of SA (Kolbe, 1860). The importance of phenolic compounds in plants, however, were ignored for another hundred years. A first hint for the importance of SA in plants came from a study on thermogenicity (conspicuous heat production in order to enhance the spread of floral odors that attract the pollinators (Meeuse and Raskin, 1988)) in voodoo lily. There it was shown that SA is able to trigger heat production upon application to the immature appendix (Raskin et al., 1987). Already in the 1960's, $\left[{ }^{14} \mathrm{C}\right]$-feeding experiments were performed in order to identify the in planta biosynthesis of SA (Klämbt, 1962; El-Basyouni et al., 1964). This led to the conclusion that SA is synthesized via phenylalanine and cinnamic acid. The name giver for this route is the first enzyme of the phenylpropanoid pathway, phenylalanine amino-lyase (PAL), which is responsible for the non-oxidative deamination of phenylalanine into transcinnamic acid. Despite intensive research on this metabolic route, in different plant species like cucumber (Métraux et al., 1990), tobacco (Malamy et al., 1990) and potato (Coquoz et al., 1998), it was not possible to decipher this pathway completely (PAL pathway Figure 3). Surprisingly, it was observed that the incorporation of ${ }^{14} \mathrm{C}$-atoms from benzoic acid or phenylalanine was lower than expected in newly formed SA (Coquoz et al., 1998). On top of that, the chemical inhibition of PAL activity did not result in a complete loss of pathogen induced SA formation in Arabidopsis (Mauch-Mani and Slusarenko, 1996). This leads to two possible conclusions, either the labeled substances were transformed into other metabolites than SA due to unexpected side reactions or there must be an alternative pathway for the synthesis of SA. When Wildermuth and colleagues analyzed the newly sequenced genome of Arabidopsis, they identified two genes, which showed high similarities to bacterial isochorismate synthase, and named them ICS1 and ICS2 (Wildermuth et al., 2001). Upon infection, the knock out mutant of ICS1, which was already found in a previous screen and was then named SA inductiondeficient 2 (sid2, (Nawrath and Métraux, 1999)), accumulated only 5-10\% of SA in comparison to wild type plants. The biosynthesis of SA from chorismate (CA) via isochorismate (ISC) is known from bacteria that use SA as a precursor of iron chelating siderophores (Walsh et al., 1990). Two mechanisms of SA biosynthesis are known in bacteria, either CA is converted into SA via a one-step reaction of a bifunctional SAsynthase (SAS, (Pelludat et al., 2003)) or in a two-step reaction. In the latter, CA is 
isomerized into ISC by an isochorismate synthase (ICS), which is followed by the action of an isochorismate pyruvate lyase (IPL). The products of the IPL-reaction are pyruvate and SA (Serino et al., 1995). When the Arabidopsis derived ICS1 was purified from heterologous expression in Escherichia coli (E. coli), Strawn and colleagues found no hints for the function as a bifunctional SAS (Strawn et al., 2007). Thus, they concluded that there must be a second enzyme in Arabidopsis, which utilizes ISC to release the final product SA (ISC pathway in Figure 3).

\section{ISC Pathway}

PAL Pathway

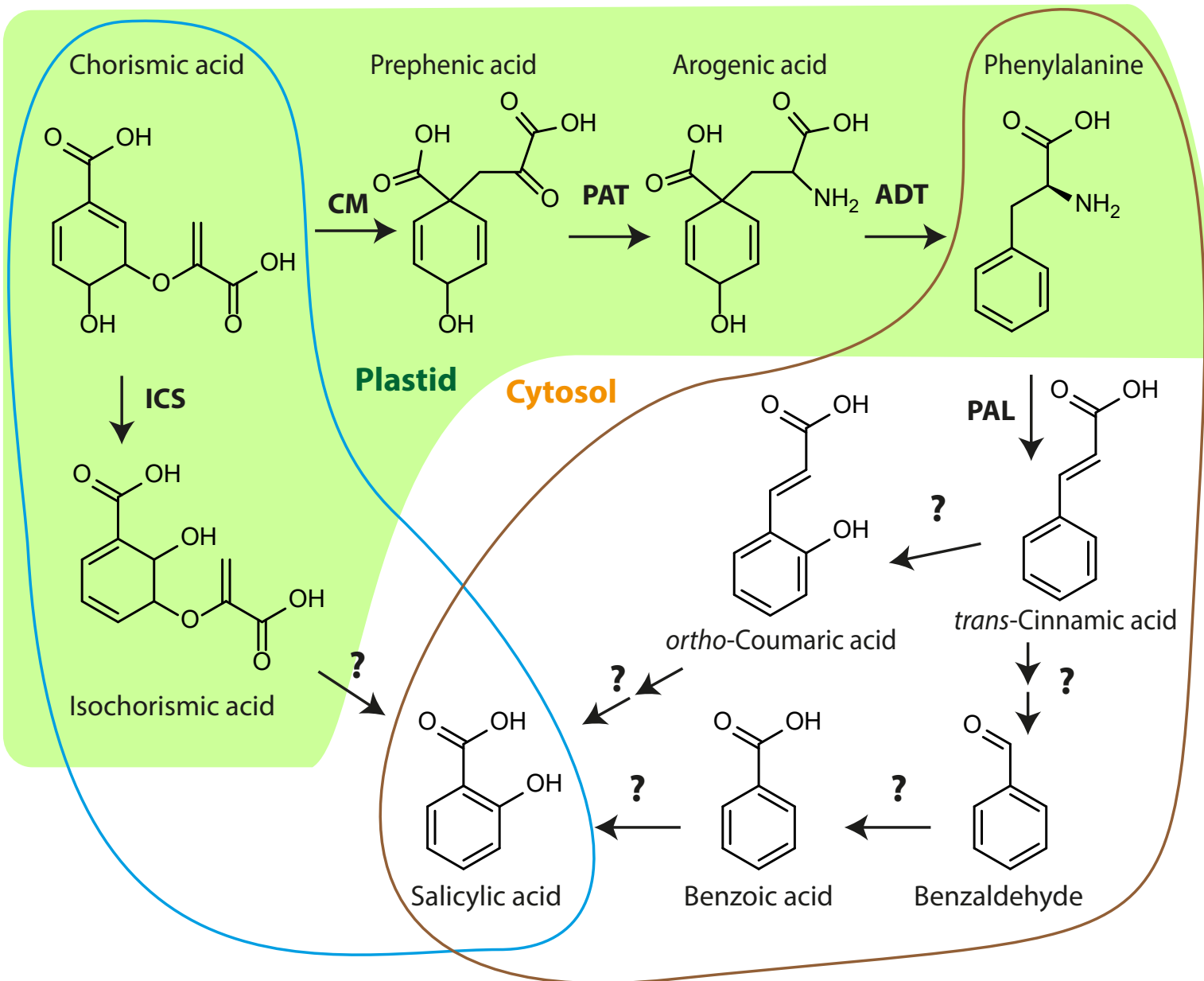

Figure 3. For the biosynthesis of salicylic acid (SA), two biosynthetic pathway were proposed for plants.

The ISC pathway (blue frame) is known from bacteria and was confirmed in C. roseus cells. The PAL pathway (brown frame) was concluded based on feeding experiments with different isotope labeled precursors. The subcellular location of the enzymatic steps are not fully understood. In the PAL pathway, phenylalanine is synthesized in plastids, whereas the PAL genes were found in the cytosol. In the ISC pathway, ICS reaction was allocated in plastids. As the in planta process for the conversion of ISC into SA is not understood yet, a proper allocation is not possible. Abbreviations: isochorismic acid (ISC), chorismate mutase $(\mathrm{CM})$, prephenate amino transferase (PAT), arogenate dehydratase (ADT), isochorismate synthase (ISC), phenylalanine ammonia lyase (PAL). Question marks represent so far uncharacterized reactions in plants.

Modified from Dempsey et al., 2011 and Maeda et al., 2011. 
ICS1 homologs are widespread throughout the plant kingdom (Dempsey et al., 2011). As ISC is required for the biosynthesis of phylloquinone, an essential component of photosystem I (Gross et al., 2006), it is conceivable that it will be present in all plant species. On the other hand, it was not possible to find an IPL enzyme in plant genomes so far, despite intensive research (Seyfferth and Tsuda, 2014; Zhou et al., 2018). A convincing proof for the existence of the ISC pathway in plants came from a study on $\left[1-{ }^{13} \mathrm{C}\right]$-Dglucose supplemented Catharanthus roseus (C. rosus) cells (Mustafa et al., 2009). Upon inoculation with Pythium aphanidermatum, a soil borne Oomycete and plant pathogen, cell cultures of $C$. roseus incorporated ${ }^{13} \mathrm{C}$-atoms into SA on specific positions, which allowed reconstructing the biosynthesis pathway. Starting from $\left[1-{ }^{13} \mathrm{C}\right]-\mathrm{D}$-glucose, the metabolic pathway through the PAL pathway would result in $\left[2,6,7-{ }^{13} \mathrm{C}\right]-\mathrm{SA}$, whereas the ISC pathway would have $[2,6-13 \mathrm{C}]-\mathrm{SA}$ as its final product. After infection of the cell cultures, Mustafa and colleagues could detect $\left[2,6^{-13} \mathrm{C}\right]-\mathrm{SA}$, but not $\left[2,6,7-{ }^{13} \mathrm{C}\right]-\mathrm{SA}$. Therefore, it can be concluded that the SA biosynthesis in C.rosus follows the proposed ISC pathway (Mustafa et al., 2009).

An interesting aspect of the SA biosynthesis is its subcellular localization. CA, the starting compound of both proposed synthetic pathways (Figure 3), is an intermediate of the shikimate pathway, which is ubiquitous in plastids of all plants. For the PAL pathway, it is known that the biosynthesis of phenylalanine takes place in the plastids (Maeda and Dudareva, 2012). In Arabidopsis, four PAL isoenzymes were described so far (PAL1-4, (Huang et al., 2010)) and none of them carries a plastid localization peptide. Phenylalanine, which is exported into the cytosol (Widhalm et al., 2015) can be subsequently utilized by these PAL-enzymes to give rise to trans-cinnamic acid. The different routes from trans-cinnamic acid to SA are poorly understood respectively the conclusions on these routes are based solely on feeding experiments (Wildermuth, 2006). Thus, neither the particular enzymes nor the location of the reaction can be stated definitively. ICS1, so far the only known enzyme of the ISC pathway, was allocated unambiguously to plastids (Strawn et al., 2007). In order to induce SA signaling, cytosolic levels of SA must rise. If SA was synthesized completely in plastids via the ISC pathway, an exporter would be required. Indeed, a promising candidate could be found with the analysis of enhanced disease susceptibility 5 (EDS5, (Glazebrook et al., 1996)). A knockout in this gene leads not only to an enhanced susceptibility to infection but also abolishes the production of pathogen triggered SA accumulation (Nawrath and Métraux, 1999). This observation appears counterintuitive, since a knockout of a transporter should lead to an 
accumulation of the cargo. Serrano and colleagues assumed in their report on this matter that SA may shut down its biosynthesis via feedback inhibition before the SA amount reaches the level of detection (Serrano et al., 2013). However, no experimental data were presented to support this statement. In order to study, if SA is actually synthesized in plastids, the SA hydroxylase gene (NahG) from Pseudomonas putida was expressed in Arabidopsis leaves, with - and without a plastid targeting signal peptide. In both cases, transgenic Arabidopsis plants, expressing NahG, failed to accumulate SA upon infection with Pseudomonas syringae respectively after UV-C stress (Fragniere et al., 2011). Taken together, it seems plausible that the ISC pathway is the major route for the biosynthesis of SA in most plants. However, as the enzymatic route from ISC to SA is not known yet, the PAL pathway must not be neglected. In Arabidopsis, the function of the four PAL genes was studied extensively. Huang and colleagues systematically produced double, triple and quadruple mutants to analyze their ability to synthesize SA (Huang et al., 2010). Neither the basal nor the pathogen induced levels of SA were influenced by any double or triple knockout combination. Even the quadruple mutants were still able to synthesize $50 \%$ of the SA that the wild type produces upon pathogen treatment. This reduction can have different reasons. One possibility is that there might be a fifth $P A L$ gene in Arabidopsis, as residual PAL activity could be measured in the quadruple mutant. However for the authors, it seems more likely that an unknown PAL downstream product is involved on a regulatory level that feeds not directly into the biosynthesis of SA (Huang et al., 2010). Possible pleiotropic effects in these mutants, however, were not discussed.

Surprisingly, only a small number of knockouts lead to a drastic reduction in pathogen induced SA biosynthesis, if general immune system hubs, like EDS1 or PAD4, and specific regulators, like SAR deficient 1 (SARD1) and Calmodulin Binding Protein 60-like.g (CBP60g, (Zhang et al., 2010b)) were not taken into account. One of those genes was found in an Arabidopsis mutants screen for susceptibility to Pseudomonas syringae pv tomato (Pst DC3000 (avrPphB)) and therefore named avrPphB susceptible 3 (PBS3, (Warren et al., 1999)). A knock out of this gene does not only lead to an enhanced susceptibility but also to a dramatic reduction of SA and its glycoside SAG. Both characteristics can be recovered by SA application, prior to inoculation (Jagadeeswaran et al., 2007; Nobuta et al., 2007). PBS3, also known as Gretchen Hagen 3.12 (GH3.12), belongs to a family of genes that was previously found in soybean to be induced upon auxin treatment (Hagen et al., 1984). GH3 enzymes are widespread throughout the plant kingdom and homologs can be found from moss to apple (Yu et al., 2018). Staswick and colleagues were the first to 
realize that GH3 enzymes belong to the firefly luciferase-like superfamily. This knowledge enabled the group to uncover that GH3.11 is responsible for the conjugation of isoleucine to JA leading to the syntheses of Ja-Ile (Staswick, 2002). Utilizing the same methods, this group also studied other members of the GH3 family (Staswick et al., 2005). PBS3 was analyzed comprehensively in a biochemical study from Okrent and colleagues (Okrent et al., 2009). Thereby, PBS3 was identified to be an acyl acid amido synthetase, with preferences for 4-hydroxybenzoic acid (4HBA) as acyl substrate and glutamic acid (Glu) as amino acid substrate. On the other hand, SA was a poor substrate and showed inhibitory properties at higher concentrations. These observations could not explain, why pbs3 mutants fail to accumulate SA. Furthermore, 4HBA-Glu, the proposed product of the in vitro reaction, was rarely found in plants (Trennheuser et al., 1994). To determine the function of PBS3 in planta, these observations are not sufficient, and thus, further studies will be required, in order to understand its connection to the SA biosynthesis.

Enhanced pseudomonas susceptibility 1 (EPS1) is another interesting gene encoding an enzyme that is potentially important for SA biosynthesis is. Similar to ics1, eds5 and pbs3, the eps1 mutants fail to accumulate SA upon pathogen challenge (Zheng et al., 2009). As for pbs3, exogenous SA application can restore the resistance against Pseudomonas infection. EPS1 belongs to the BAHD acyltransferase superfamily (Tohge et al., 2018). BAHD enzymes catalyze CoA-dependent acyl transfers of plant secondary metabolites, including anthocyanins and flavonoids. Since no substrate of EPS1 has been identified so far, it remains elusive how EPS1 might influence the biosynthesis of SA.

\subsection{Pipecolic acid}

Although the importance of Pip for the plant immunity was discovered only seven years ago (Navarova et al., 2012), its presence in plants has been known since several decades (Zacharius et al., 1952; Morrison, 1953). Again, isotope feeding experiments were instrumental to study the biosynthetic route. Lysine was identified as the precursor of Pip not only in plants but also in mammals (Gupta and Spenser, 1969). Two competing routes for the conversion of lysine into Pip were proposed (Figure 4). The main difference between these routes is, whether the $\alpha$ - or the $\varepsilon$-nitrogen of lysine is finally incorporated into Pip (Schütte and Seelig, 1967). In rats, Rothstein and colleagues showed that only the radioactive labeled nitrogen from the $\varepsilon$-position of lysine leads to labeled Pip (Rothstein and Miller, 1954). Hence, it was proposed that Pip is synthesized through an $\alpha$ - 
aminotransferase, which utilizes lysine to produce $\alpha$-keto- $\varepsilon$-aminocaproic acid, which spontaneously cyclizes in solution to $\Delta^{1}$-piperideine-2-carboxylic acid (P2C, (Meister, 1954)). Subsequently, the ketimine P2C is then reduced to yield the final product Pip. Contrarily, when similar feeding experiments were performed with green bean, Schütte and colleagues found that it was the nitrogen at the $\alpha$-position, which was incorporated into Pip (Schütte and Seelig, 1967). Previously, an amine oxidase activity was found in pea, which was able to utilize lysine. The product of this reaction cyclized in solution and could finally be converted into Pip by hydrogenation (Mann, 1955). Therefore, it was conceivable that this amine oxidase removes the $\varepsilon$-nitrogen to yield $\alpha$-aminoadipate- $\delta$ semialdehyde, which, comparable with $\alpha$-keto- $\varepsilon$-aminocaproic acid, cyclizes to $\Delta^{1}$ piperideine-6-carboxylic acid (P6C). Upon reduction, P6C can be subsequently transformed into Pip (Schütte and Seelig, 1967). Contradictory to these results, Gupta and co-workers presented a comprehensive study revealing that Pip was produced via P2C and not P6C (Gupta and Spenser, 1969). Another route to reach P6C from lysine goes through saccharopine, which is originally characterized in mammals (Higashino et al., 1971). In fungi, this route is used for the biosynthesis of lysine instead of its degradation (Jones and Broquist, 1966). Feeding experiments with different labeled precursors in barley led to the conclusion that lysine is partially catabolized via saccharopine into P6C and Pip in plants as well (Møller, 1976). For the biosynthesis of saccharopine in plants, lysine-ketoglutarate reductase (LKR) is required, which condenses lysine with $\alpha$ ketoglutarate. A saccharopine dehydrogenase (SDH) subsequently splits its substrate into $\alpha$-aminoadipate- $\delta$-semialdehyde and Glu (Galili et al., 2001). In plants, these enzymes are encoded by a bifunctional polypeptide (LKR/SHD, (Gonçalves-Butruille et al., 1996)). The final fate of $\alpha$-aminoadipate- $\delta$-semialdehyde is poorly understood, since a dehydrogenase, has not been found in plants so far, which would catalyze the reaction into $\alpha$-aminoadipic acid (Figure 4). Upon infection, Arabidopsis accumulates significant amounts of Pip in treated as well as in systemic leaves. This accumulation is abolished completely in knockout mutants of AGD2-like defense response protein 1 (ALD1, (Navarova et al., 2012). In a previous report, ALD1 showed aminotransferase activity with lysine but also on other amino acids (Song et al., 2004a). Whether ALD1 catalyzes the first step of the $\mathrm{P} 2 \mathrm{C}$ or the $\mathrm{P} 6 \mathrm{C}$ route requires further clarification. Based on the results from Gupta et al. 1969, Navarova and coworkers suggested that there is a P2C route in plants. It is known from mammalian systems that P2C exists in tautomeric equilibrium of its 
ketamine and enamine form (Hallen et al., 2015). It is unknown yet, which of these tautomers is further reduced into Pip.

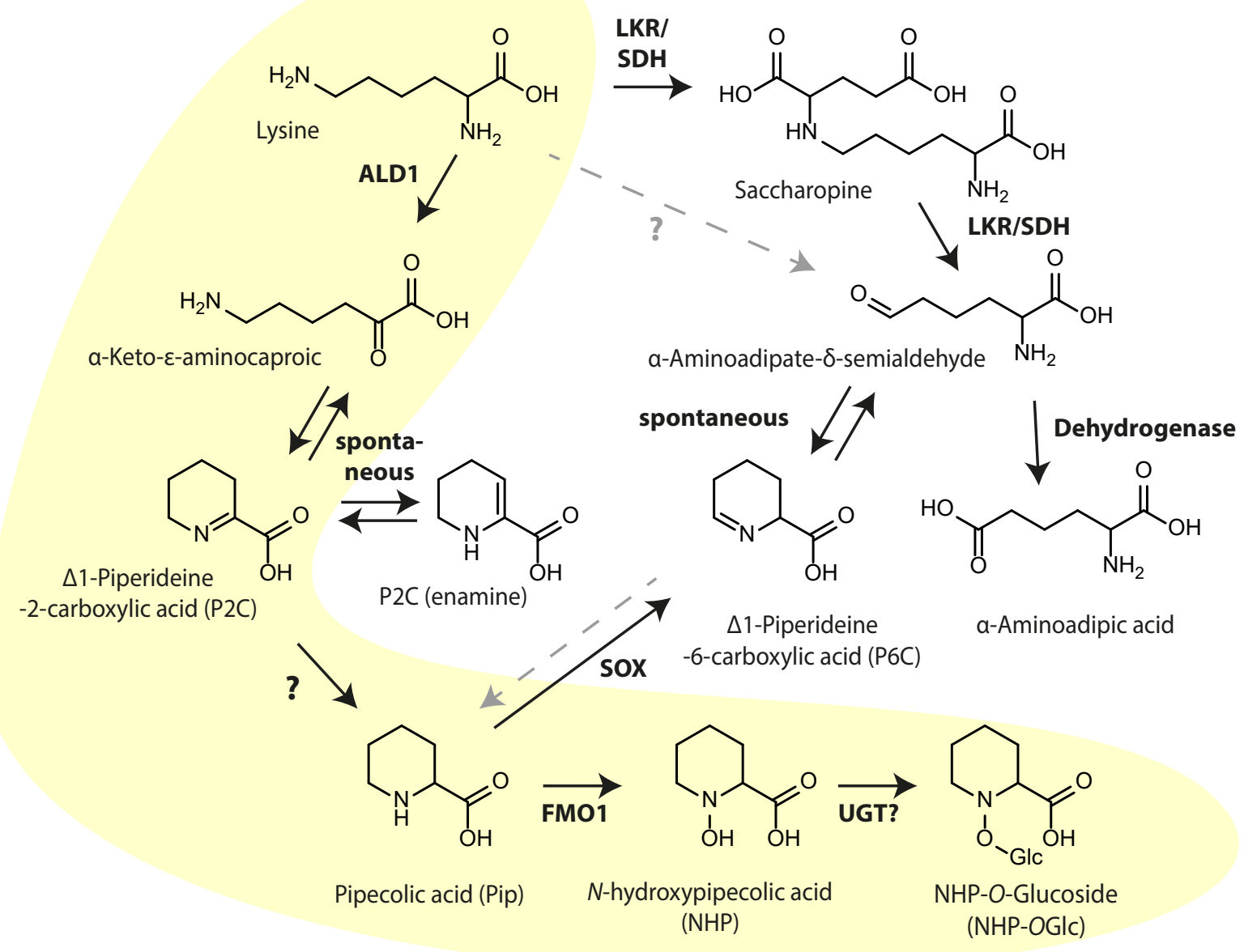

Figure 4. Two routes of lysine dependent pipecolic acid (Pip) biosynthesis.

In plants, lysine degradation leads either to the formation of pipecolic acid (Pip) or to $\alpha$-aminoadipic acid. The pathogen induced route in plants is highlighted in yellow. In order to generate Pip, one nitrogen of lysine needs to be removed. According to Guptal et al., 1969 plants prefer the route via $\alpha$ keto- $\varepsilon$-aminocaproic acid, which cyclizes spontaneously to $\Delta^{1}$-piperideine-2-carboxylic acid (P2C). In solution, $\mathrm{P} 2 \mathrm{C}$ occurs in tautomeric equilibrium between ketimine and enamine form (Hallen et al., 2015). P2C is finally converted into Pip by a so far unknown ketimine reductase. To reach $a-$ aminoadipate- $\delta$-semialdehyde from lysine, either an $\varepsilon$-transaminase - or lysine-ketoglutarate reductase (LKR) with additional saccharopine dehydrogenase (SDH) would be required. So far, only the LKR/SDH route was explored in plants. $\alpha$-Aminoadipate- $\delta$-semialdehyde is further converted towards $\alpha$ aminoadipic acid via an unknown dehydrogenase, or it cyclizes spontaneously to $\Delta^{1}$-piperideine-6carboxylic acid (P6C). It is not clear, whether P6C can be converted into Pip as only the reverse reaction was found so far to be catalyzed by a sarcosine oxidase (SOX, (Goyer et al., 2004)). Recently, it was shown that Pip is further metabolized by flavin-dependent monooxygenase 1 (FMO1) into $N$-hydroxy pipecolic acid (NHP, (Hartmann et al., 2018)). Subsequently, NHP is converted by a yet unknown UDPdependent glycosyltransferase into NHP-O-Glucoside (NHP-OGlc, (Chen et al., 2018)). For the first step of the $\mathrm{P} 2 \mathrm{C}$ route, the aminotransferase AGD2-like defense response protein 1 (ALD1) was proposed (Navarova et al., 2012). Modified from Hartmann and Zeier, 2018 and Hallen et al., 2015. 
Flavin-dependent mono-oxygenase 1 (FM01) is another important gene for the plant immune system. A mutation in this gene leads to a higher susceptibility against virulent Pseudomonas strains. On the other hand, lines over expression FM01 had an enhanced resistance against different types of infection (Koch et al., 2006). In addition to the basal resistance, the establishment of SAR is also disturbed in fmo1 plants (Mishina and Zeier, 2006). Upon Pseudomonas infection, Navarova and colleagues observed that Pip accumulates even stronger in fmo1 - than in the wild type plants, suggesting that Pip might be the substrate of FM01 (Navarova et al., 2012). FMOs are known to transfer hydroxyl groups onto heteroatomic substrates, which for instance contain nitrogen (Franceschini et al., 2012) or sulfur (Hansen et al., 2007). Thus, it is not surprising that FM01 was identified as an enzyme that hydroxylates Pip at the nitrogen position to yield NHP ((Hartmann et al., 2018), Figure 4). NHP seems to be a more potent inducer of plant immunity than Pip against a battery of pathogens. Additionally, NHP pretreatment induce priming in untreated tissues and thus, NHP was characterized as a critical regulator of SAR (Chen et al., 2018). Similar to Pip, also NHP seems to be widely spread throughout the plant kingdom (Holmes et al., 2019). Future research will show, if NHP has a similar role in plant immunity of other plants than Arabidopsis. In addition to NHP, Chen and colleagues found a glycosylated form of NHP, and showed that this NHP-O-Glucoside (NHP-OGlc) also accumulates after infection (Chen et al., 2018). Glycosylation is a common modification of signaling compounds (Haroth, 2018). Glycosides often have a higher solubility and thus accelerate the transport of their aglycones to a different interor intracellular localization (Dean et al., 2005). The influence of the glycosylation on NHP and its bioactivity needs further analysis. Glycosylation of signal compounds is often facilitated by UDP-dependent-glycosyltransferases (UGTs). These widespread enzymes transfer sugar moieties from UDP-activated sugars onto signaling molecules as well as to a huge variety of specialized metabolites (Vogt and Jones, 2000). It remains to be determined, which enzyme of the UGT-super family is responsible for the formation of NHP-OGlc (Lim et al., 2002). Similar to the biosynthesis of SA, also Pip synthesis was also allocated to plastids (Hartmann et al., 2018). FM01 however does not have a plastid localization peptide. Its exact localization is not determined yet, but based on homology to the yeast FMO, it is conceivable that FM01 may also attach to the outer surface of the ER (Zhang and Robertus, 2002). Thus, the difficulties occurring during the protein purification of heterologously expressed FM01 could be explained by this specific membrane association (Chen et al., 2018). Additionally, the vast majority of UGTs were 
allocated to the cytosol (Li et al., 2001). This means that the biosynthesis of Pip occurs in a different subcellular compartment than the hydroxylation by FM01 and the subsequent glycosylation to NHP-OGlc. Thus, a yet unknown plastidial transporter must exist that transfers Pip from plastids into the cytosol. In Figure 5, different aspects of plant immunity discussed in the sections above are depicted.

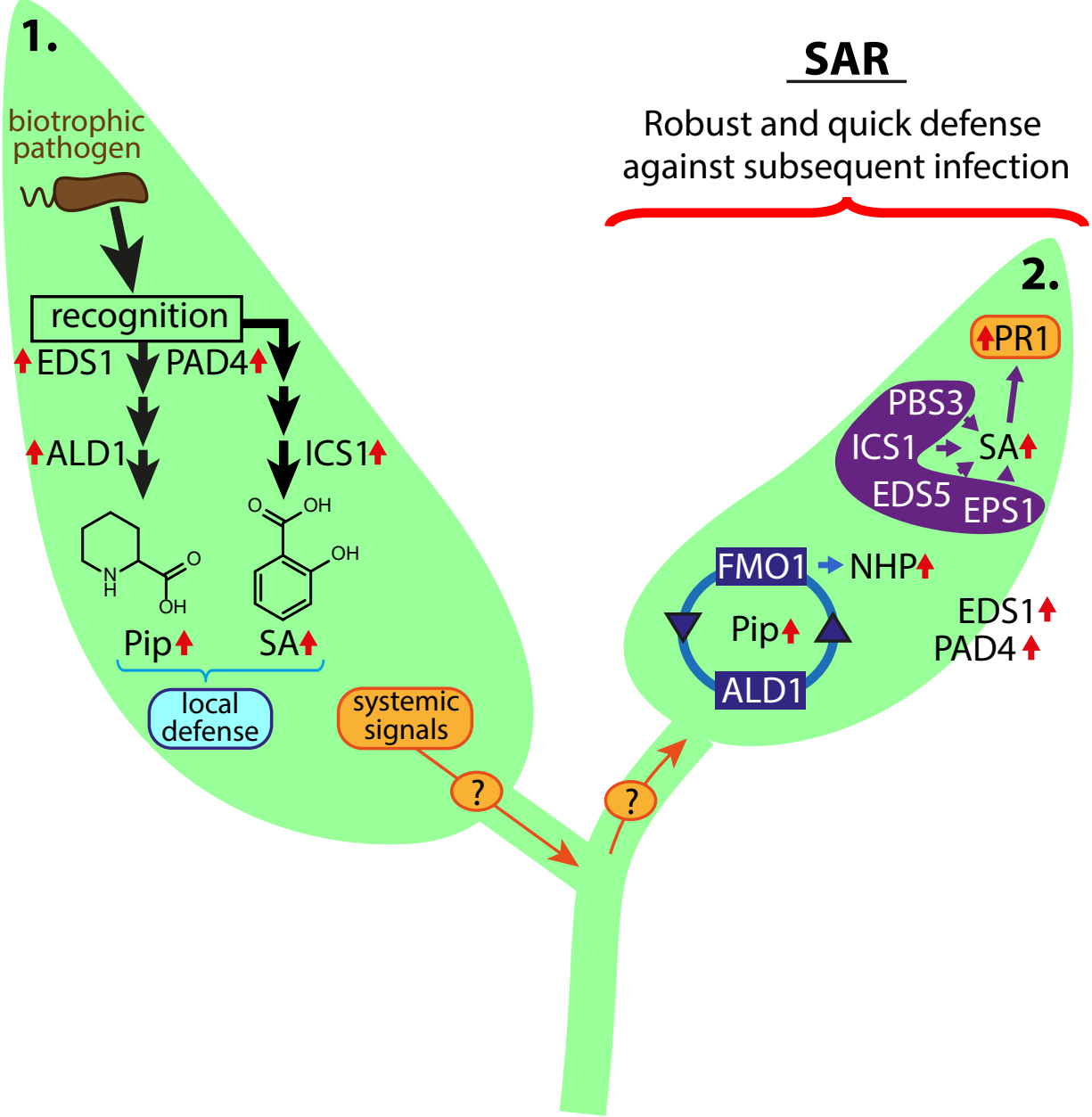

Figure 5. Current model for the induction of systemic acquired resistance (SAR) by biotrophic pathogens.

Upon infection of biotrophic pathogens, several layers of plant immunity are activated. The recognition of the invader activates central regulators of the plants defense, e.g. EDS1 and PAD4. This stimulates, along with other metabolic pathways, the biosynthesis of the signaling compounds SA and Pip. Prominent enzymes in the biosynthesis of these metabolites are ALD1 for Pip and ICS1 for SA. Together, these compounds coordinate the local defense against the invader. In addition to the basal response, not fully understood chemical signals are produced, which spread through the vascular system towards distal tissues. In the systemic tissues, these signals induce changes in histone modifications, gene expression and metabolic alterations. Again the synthesis of Pip and SA is facilitated by different enzymes. The accumulation of SA can be monitored by the expression of the marker gene PR1. SA and Pip, respectively, the Pip derived product of FMO1 NHP, facilitate the establishment of SAR.

Abbreviations: enhanced disease susceptibility 1 (EDS1), phytoalexin deficient 4 (PAD4), salicylic acid (SA), pipecolic acid (Pip), AGD2-like defense response protein 1 (ALD1), isochorismate synthase 1 (ICS1), pathogenesis-related genes 1 (PR1), flavin-dependent monooxygenase 1 (FMO1), $N$-hydroxy pipecolic acid (NHP). Modified from Navarova et al., 2012. 
Thereby, the central role of SA and Pip in the basal resistance as well as in the establishment of SAR is highlighted. It should be mentioned here that there is an ongoing debate if plant immunity is generally SA regulated or if there is also an SA independent branch (Mishina and Zeier, 2006). Based on the observations that the double mutant ald1sid2 shows a stronger SAR deficiency phenotype than the individual single mutants, it was proposed that Pip might be the signaling compound that regulates the SA independent plant immunity (Bernsdorff et al., 2016). Interestingly, local treatment with Aza or G3P restores the ability of ald1 plants to establish SAR in distal leaves, whereas SA treatment had no significant improvements. In addition, priming with Pip did not improve resistance in mutants defective in Aza signaling. Therefore, Wang and colleagues proposed that Pip acts upstream of Aza signaling and thereby confers systemic resistance independent of SA (Wang et al., 2018). These results emphasize the outstanding role of Pip respectively NHP in the field of SAR and plant immunity.

\subsection{Metabolomics in plant immunity}

Plants evolved a variety of strategies to adapt to biotic and abiotic stress, as they cannot escape from these threats since they are sessile organisms. One of the key strategies to cope with environmental alterations is the ability to produce a functionally and structurally highly diverse metabolic network (Feussner and Polle, 2015). Within this mixture of metabolites small common compounds, like proline that can act as an osmolyte or metal chelator (Hayat et al., 2012), as well as highly specific metabolites, like tomatine and other phytoalexines (Piasecka et al., 2015), need to be present in appropriate amounts. In order to survive, plants adapt constantly and rapidly to the physiological changes (Tugizimana et al., 2018). The massive changes in gene expression upon pathogenic attacks were mentioned in section 1.2. The enhanced expression of regulatory genes, however, regulates a large number of biochemical processes, which finally might end up in an even larger number of produced metabolites. For specialized metabolites, the number of possible modifications on a core structure is drastically enlarged due to substrate promiscuity of enzymes responsible for these modifications (Weng, 2014). This means that understanding of metabolic changes upon stress bundles the information of upstream control levels. In order to holistically grasp a multilayered process like the plant microbe interaction or the establishment of SAR, it is therefore important to study changes on the metabolic level. 
In metabolomics, changes in metabolite levels can be studied either by targeted or by untargeted approaches (Figure 6). In both cases, liquid - or gas chromatography (LC or GC) may be used upstream of a mass spectrometer (MS) in order to separate metabolites within complex samples. Subsequently, the molecules passing the chromatography are ionized and analyzed in the MS (Smith et al., 2014). Alternatively, targeted approaches sometimes use optical detectors, such as a photodiode array (PDA), instead of MS in order to detect and quantify particular metabolites. In a targeted approach, a predefined number of metabolites is analyzed and the use of internal standards enables quantifications. The extraction procedures, the chromatographic separation as well as detection procedure has to be optimized to the characteristics of the metabolites of interest. To enhance the sensitivity, targeted LC/MS based metabolomics setups often use multiple reaction monitoring (MRM). Thereby, studies utilize triple quadrupole MS to achieve a fast and robust measurement. In the first quadrupole ionized molecules of interest are isolated by their mass-to-charge ratio $(\mathrm{m} / \mathrm{z})$. In the second quadrupole the

a Targeted metabolomics

Goal: Detect and quantify alterations of predefined metabolites

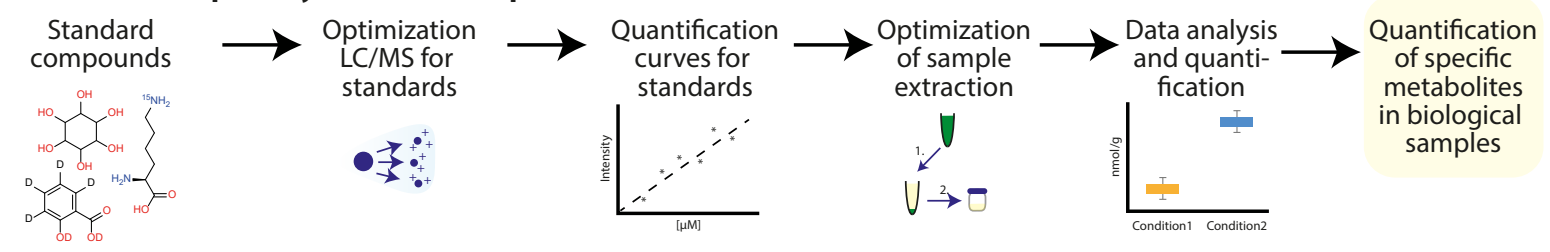

b Untargeted metabolomics

Goal: Depict alterations of the global metabolic profile and identify marker

$\begin{gathered}\text { General } \\ \text { metabolite } \\ \text { extraction }\end{gathered}$
$\begin{gathered}\text { LC/MS methode } \\ \text { for broad range } \\ \text { of metabolites }\end{gathered}$$\quad \begin{gathered}\text { Detection of } \\ \text { all ions from } \\ \text { one LC/MS run }\end{gathered} \rightarrow \begin{gathered}\text { Data aligment } \\ \text { and analysis }\end{gathered} \rightarrow \begin{gathered}\text { Validation and/or } \\ \text { structure eluci- } \\ \text { dation with MS/MS }\end{gathered}$

Figure 6. Schematic representation of targeted and untargeted metabolomics pipelines.

Comparison of targeted and untargeted metabolomic workflows utilizing liquid chromatography followed by mass spectrometry (LC/MS). (a) In the targeted set up, only a predefined number of compounds is analyzed in order to quantify changes of metabolites of interest. The limited number of targets allows an optimization of the extraction and analysis procedure. Comparisons with internal standards allow an adequate quantification. (b) The untargeted workflow enables the study of all compounds within one sample. The large number of ions detected in this setup demands an extensive computational data analysis. In order to validate tentatively identified compounds, either comparison with authentic standards or tandem mass spectrometry (MS/MS) fragmentation are employed. The fragmentation pattern can subsequently be matched to MS/MS databases, in order to confirm the identity. New computational tools might simplify structure elucidation in near future.

Modified from Patti et al., 2012. 
targeted ion is fragmented with a compound specific voltage. Lastly, the fragment ions are transferred into the third quadrupole, in which the signal intensity of a diagnostic fragment (of a particular $m / z$ ), is monitored by the detector. In this way, the identity of the compound can be ensured as metabolites have specific precursor/product ion pairs, which, when combined with a predefined retention time on the chromatography, allow an unambiguous identification (Roberts et al., 2012). Currently, even a second precursor/product ion pair is often used to confirm the correct identification. This setup is for instance very useful for analyzing the concentration of phytohormones, where already changes within a certain subset of metabolites might lead to dramatic phenotypes (Bernsdorff et al., 2016). Untargeted metabolomics, on the other hand, enables to monitor the alteration of well-known metabolite markers as well as the detection of new and so far unidentified metabolites or even metabolic pathways, which are of particular importance for specific processes like the establishment of SAR.

Untargeted measurements results in the detection of several thousand metabolite features. These features correspond to a detected ion with an accurate $m / z$, a particular retention time in the chromatography and the corresponding intensity pattern over all samples analyzed in a sample set (Kaever et al., 2012). The large number of detected features is boon and bane alike. The plant metabolome was estimated to contain approximately 200000 distinct metabolites. Depending on the species, 5000-25000 compounds can be found in an individual plant (Creydt and Fischer, 2017). On the other hand, there are only 5000 commercially available compounds, which can be used for unambiguous identifications. Thus, a typical untargeted metabolomics study may monitor the variation in 100-150 metabolites, including compounds with unclear identity (Lassowskat et al., 2014).

The number of detected ions within an untargeted investigation is too large for manual analysis. Thus, computational analysis is employed for peak picking and peak alignment, as well as subsequent data deisotoping and deconvolution (Smith et al., 2014). To further reduce the number of features, statistical analysis is employed to obtain marker candidates, which are specific for the particular research question. Features with significant changes in their accumulation pattern, e.g. being only present in wild type, but not in a mutant, and high reproducibility, similar amount in each sample of one category, can be ranked based on statistical relevance (Kaever et al., 2012). For the best-ranked marker candidates, a tentative computational metabolite identification based on the precise mass information would be desirable. 
Matching the mass information with online - or custom databases is often not sufficient for an unambiguous identification. A helpful strategy to overcome this problem is to employ so called metabolite set enrichment analysis. Thereby, mass information is combined with the metabolic pathway maps, which can be found in the Kyoto Encyclopedia of Genes and Genomes (KEGG, (Kanehisa et al., 2012)) and MetaCyc (Caspi et al., 2012), in order to identify pathways that contain an enriched number of features. The missing metabolites of such pathways may be lost during data processing but can be frequently found in the raw data (Kaever et al., 2015). As mentioned previously, plants often produce a metabolite core structure, which can have various sorts of modifications. Therefore, in silico data base extensions originating from known core structures can support the data mining significantly. Despite this variety of strategies, automatic identification is poorly established so far and represents the major bottleneck of untargeted metabolomics (Tugizimana et al., 2018). The previously mentioned low number of commercial standards makes the situation even more difficult. Thus, for the elucidation of the chemical structure two strategies, Nuclear Magnetic Resonance spectroscopy (NMR) or tandem mass spectrometry (MS/MS), are commonly used (Boiteau et al., 2018). Both methods have their advantages and disadvantages (Emwas, 2015). However, a comprehensive database is available for none of these methods. In their commentary on the state of metabolomics, da Silva and colleagues discussed that less than two percent of the spectra, detected in an untargeted metabolomics investigation, can be properly annotated (da Silva et al., 2015). This is due to the low number of compounds ( 20000, (Guijas et al., 2018)), which have a fragmentation spectra in a reference database like METLIN (Smith et al., 2005) or MassBank (Horai et al., 2010), in comparison to the over 100 million entries in the molecular structure database PubChem (Ludwig et al., 2018). In order to overcome these difficulties, a large number of computational tools can be used (Misra and Mohapatra, 2019). These programs automatically compare experimental fragmentations with reference databases in order to predict common structures with additional machine learning processes (Ludwig et al., 2018). Such in silico fragmentations show some success in the identification of so far non-characterized metabolites (Schymanski et al., 2017) and may be instrumental for the future of metabolomics studies.

Despite the drawbacks in the metabolite annotation, untargeted metabolomics is indispensable for the understanding of complex processes. Application of this method led to discovery of key components of plant immunity like Aza (Jung et al., 2009), DA 
(Chaturvedi et al., 2012), and pinenes (Riedlmeier et al., 2017). Additionally, metabolite fingerprinting analyses was employed to study global changes upon activation of MTI (Lassowskat et al., 2014; Finnegan et al., 2016), different sorts of priming (Balmer et al., 2015), plant-pathogen interactions (Konig et al., 2014; Galeano Garcia et al., 2018) and beneficial plant-microbe interactions (Stringlis et al., 2018). Taking these reports together, it becomes obvious how important and indispensable metabolomics became for the understanding of plant immunity. On top of that, the large number of unknown compounds in untargeted studies provide opportunities to verify newly discovered compounds on their availability in planta (Hamberg and Gardner, 1992; Staswick and Tiryaki, 2004).

\subsection{Aims of the study}

The presence of an innate immune system in plants has been known since several decades (Chester, 1933; Ross, 1961; Jones and Dangl, 2006; Cook et al., 2015). Despite great advances in the last decade, our understanding of the plant immunity is far from being complete. The plant-pathogen interaction is orchestrated by a specific subset of plant signaling compounds that regulate downstream processes and can spread throughout the plant in order to alarm uninfected tissues (Kachroo and Robin, 2013). The overall focus of this work was to study and to complete metabolic pathways that are essential for the plant immunity in general and for the establishment of SAR in particular in the model plant Arabidopsis thaliana.

The successful immune response of plants against biotrophic pathogens strongly depends on the signaling compounds SA and Pip/NHP (Hartmann et al., 2018). Although their key role is widely accepted, the biosynthesis of these metabolites is only partially understood (Dempsey et al., 2011; Hartmann and Zeier, 2018). In order to contribute to this open question, metabolite fingerprint analysis combined with protein biochemistry and genetics-driven mutant screens were used extensively. Thereby, metabolic alterations between wild type and different immune deficient mutant plants had to be analyzed in a comprehensive untargeted way to identify missing links of the SA und Pip/NHP metabolic pathways. In particular, the ald1 and sard4 mutant plants were investigated in order to understand Pip/NHP biosynthesis (Article I), whereas the eds5, sid2 and pbs3 mutants were utilized to study SA biosynthesis (Article II). 
In order to verify the results gathered in the untargeted metabolomics approach, heterologous expression and purification of ALD1, SARD4, ICS1 and PBS3 had to be established for in vitro activity assays with the pure enzymes. Using this approach, the biosynthesis of both Pip/NHP and SA could be elucidated unambiguously. For both metabolic pathways, the subcellular distribution seems to represent an important level of regulation (Dempsey et al., 2011; Hartmann and Zeier, 2018). Therefore, the cellular location of the different reaction steps responsible for the synthesis of SA and NHP was analyzed (Article III). 


\section{Article I. Characterization of a Pipecolic Acid Biosynthesis Pathway Required for Systemic Acquired Resistance}

This article was published online in The Plant Cell in October 2016. The supplementary materials are attached to the main part. The full article can be found online:

https://doi.org/10.1105/tpc.16.00486

\section{Author contribution:}

Dmitrij Rekhter planned and performed the metabolite fingerprint analysis as well as the subsequent metabolite identification by high resolution UHPLC-Q-TOF-MS/MS analysis. Moreover, he amplified, cloned, expressed and established the purification of AtALD1 and AtSARD4. He performed the in-cell activity assay and the in vitro studies with the purified enzymes. He analyzed, processed, displayed and discussed the data resulting from those experiments, and wrote the corresponding parts of the manuscript (including Figures 4, 6-8, S4-7). 


\title{
Characterization of a Pipecolic Acid Biosynthesis Pathway Required for Systemic Acquired Resistance
}

\author{
Pingtao Ding, ${ }^{\mathrm{a}, 1}$ Dmitrij Rekhter, ${ }^{\mathrm{b}, 1}$ Yuli Ding, ${ }^{\mathrm{a}, 1}$ Kirstin Feussner, ${ }^{\mathrm{b}}$ Lucas Busta, ${ }^{\mathrm{c}}$ Sven Haroth, ${ }^{\mathrm{b}}$ Shaohua Xu, ${ }^{\mathrm{d}}$ Xin Li, ${ }^{\mathrm{a}}$ \\ Reinhard Jetter, ${ }^{a, c}$ Ivo Feussner, ${ }^{b, e, 2}$ and Yuelin Zhang ${ }^{a, 2}$ \\ a Department of Botany, University of British Columbia, Vancouver BC V6T 1Z4, Canada \\ b Department of Plant Biochemistry, Georg-August-University, Albrecht-von-Haller-Institute for Plant Sciences, D-37073 Goettingen, \\ Germany \\ c Department of Chemistry, University of British Columbia, Vancouver BC V6T 1Z4, Canada \\ dNational Institute of Biological Sciences, Beijing 102206, China \\ e Department of Plant Biochemistry, Georg-August-University, Goettingen Center for Molecular Biosciences, D-37073 Goettingen, Germany \\ ORCID IDs: 0000-0002-3535-6053 (P.D.); 0000-0002-9888-7003 (I.F.); 0000-0002-3480-5478 (Y.Z.)
}

\begin{abstract}
Systemic acquired resistance (SAR) is an immune response induced in the distal parts of plants following defense activation in local tissue. Pipecolic acid (Pip) accumulation orchestrates SAR and local resistance responses. Here, we report the identification and characterization of SAR-DEFICIENT4 (SARD4), which encodes a critical enzyme for Pip biosynthesis in Arabidopsis thaliana. Loss of function of SARD4 leads to reduced Pip levels and accumulation of a Pip precursor, $\Delta^{\mathbf{1}}$ piperideine-2-carboxylic acid (P2C). In Escherichia coli, expression of the aminotransferase ALD1 leads to production of P2C and addition of SARD4 results in Pip production, suggesting that a Pip biosynthesis pathway can be reconstituted in bacteria by coexpression of ALD1 and SARD4. In vitro experiments showed that ALD1 can use L-lysine as a substrate to produce P2C and P2C is converted to Pip by SARD4. Analysis of sard4 mutant plants showed that SARD4 is required for SAR as well as enhanced pathogen resistance conditioned by overexpression of the SAR regulator FLAVIN-DEPENDENT MONOOXYGENASE1. Compared with the wild type, pathogen-induced Pip accumulation is only modestly reduced in the local tissue of sard4 mutant plants, but it is below detection in distal leaves, suggesting that Pip is synthesized in systemic tissue by SARD4-mediated reduction of P2C and biosynthesis of Pip in systemic tissue contributes to SAR establishment.
\end{abstract}

\section{INTRODUCTION}

Systemic acquired resistance (SAR) is an evolutionarily conserved defense mechanism induced in the distal parts of plants after a locally restricted primary infection (Fu and Dong, 2013). Following local infection, mobile signals are generated in inoculated leaves and transported to other parts of the plant. Perception of the signals in the systemic tissue leads to activation of long-lasting protection against a broad spectrum of microbial pathogens. Salicylic acid (SA) is required for both SAR and local defense responses but is unlikely to function as a critical long distance signal in SAR (Vlot et al., 2009). Several metabolites including methyl salicylate, azelaic acid, dehydroabietinal, and a molecule derived from glycerol-3-phosphate have been shown to be involved in long distance signaling during SAR (Park et al., 2007; Jung et al., 2009; Chanda et al., 2011; Chaturvedi et al., 2012). The lipid transfer proteins DEFECTIVE IN INDUCED RESISTANCE1 and AZELAIC ACID INDUCED1 play critical roles in long-distance signaling mediated by some of these metabolites (Maldonado et al., 2002; Jung et al., 2009; Champigny et al., 2013; Yu et al., 2013).

\footnotetext{
${ }^{1}$ These authors contributed equally to this work.

${ }^{2}$ Address correspondence to ifeussn@gwdg.de or yuelin.zhang@ubc.ca. The authors responsible for distribution of materials integral to the findings presented in this article in accordance with the policy described in the Instructions for Authors (www.plantcell.org) are: Yuelin Zhang (yuelin.zhang@ubc.ca) and Ivo Feussner (ifeussn@gwdg.de). www.plantcell.org/cgi/doi/10.1105/tpc.16.00486
}

Several genes encoding putative enzymes that are mainly related to amino acid metabolism had been found to play important roles in plant defense responses, suggesting that additional signal molecules are required for plant defense against pathogens (Zeier, 2013). Among them, ALD1 encodes an aminotransferase and FLAVIN-DEPENDENT MONOOXYGENASE1 (FMO1) encodes a putative flavin-dependent monooxygenase. ALD1 and FMO1 are required for SAR as well as local defense (Song et al., 2004a; Bartsch et al., 2006; Koch et al., 2006; Mishina and Zeier, 2006; Bernsdorff et al., 2016). Loss of function of ALD1 results in increased susceptibility to both virulent and avirulent pathogens and SAR deficiency. Overexpression of FMO1 leads to increased resistance against virulent pathogens, whereas loss of function of FMO1 leads to enhanced susceptibility to pathogens and complete loss of SAR (Bartsch et al., 2006; Koch et al., 2006; Mishina and Zeier, 2006). Pathogen resistance mediated by FMO1 had been shown to be independent of SA (Bartsch et al., 2006; Bernsdorff et al., 2016).

Analysis of amino acid metabolism following pathogen infection showed that ALD1 is required for the biosynthesis of pipecolic acid (Pip), which is an intermediate of lysine degradation (Návarová et al., 2012). Infection by Pseudomonas syringae pv maculicola (P.s.m.) ES4326 induces a strong increase in Pip accumulation in the wild type, but not in ald1 mutant plants. Defects in basal resistance and SAR in ald1, but not in fmo1 mutants, can be complemented by exogenous application of Pip, suggesting that lack of Pip production is responsible for the immune deficiency in ald1. 
Pretreatment with Pip leads to increased pathogen resistance and induces SAR-related defense priming in wild-type plants, suggesting that Pip functions as a critical regulator of inducible plant immunity (Návarová et al., 2012). However, whether Pip moves systemically during infection is unknown.

Previously we developed a high-throughput "brush and spray" assay for SAR and used it to carry out a forward genetic screen to search for SAR-deficient mutants (Jing et al., 2011). Among the mutants with strong SAR deficiency phenotypes, six are alleles of fmo1, four are alleles of ald1, and three are alleles of $S A$ INDUCTION DEFICIENT2, highlighting the importance of SA and metabolites synthesized by FMO1 and ALD1 in SAR. Here, we report the identification and characterization of SAR DEFICIENT4 (SARD4), which encodes an enzyme involved in the final step of Pip biosynthesis.

\section{RESULTS}

\section{Identification of sard4 Mutants}

In a previously described forward genetic screen for SARdeficient mutants (Jing et al., 2011), two sard4 alleles were identified. As shown in Figure 1A, both sard4-1 and sard4-2 displayed compromised SAR. The mock-treated sard4-1 and sard4-2 also appeared to be more susceptible to Hyaloperonospora arabidopsidis (H.a.) Noco2. In a separate genetic screen to identify genes required for enhanced basal resistance conditioned by overexpression of FMO1, FMO1-3D, an FMO1 overexpression mutant identified by activation tagging (Koch et al., 2006), was mutagenized with EMS. Screening $\sim 45,000$ M2 plants representing $\sim 3000 \mathrm{M} 1$ families for compromised resistance against H.a. Noco2 identified two mutants shown to be different alleles of sard4. They were named sard4-3 and sard4-4. As shown in Figure 1B, enhanced resistance against H.a. Noco2 in FMO1-3D is largely suppressed by sard4-3 and sard4-4. In FMO1-3D, the defense marker genes $P R 1$ and $P R 2$ are constitutively expressed. The elevated expression of $P R 1$ and $P R 2$ is largely suppressed in sard4-3 FMO1-3D and sard4-4 FMO1-3D (Figures $1 \mathrm{C}$ and 1D).

\section{SARD4 Encodes a Protein Similar to Bacterial Ornithine Cyclodeaminase}

The sard4-3 and sard4-4 mutations were initially mapped to a region between marker K19E20 and MMN10 on chromosome 5. Further mapping of sard4-3 narrowed the mutation to a region between markers K10D11 and MYN8. In this region, At5g52810 encodes a protein with similarity to bacterial ornithine cyclodeaminase and it is induced by pathogen infection based on the TAIR microarray database. Sequencing At5g52810 in sard4-3 identified a G-to-A mutation in the gene, which results in a Gly-89to-Glu amino acid substitution. Sequencing the At5g52810 locus in the sard4-1, sard4-2, and sard4-4 mutants showed that they all contain nonsynonymous mutations in the gene (Figure 2A), suggesting that At5g52810 is SARD4. Quantitative RT-PCR analysis confirmed that $A t 5 g 52810$ is induced by P.s.m. ES4326 (Figure 2B).
A

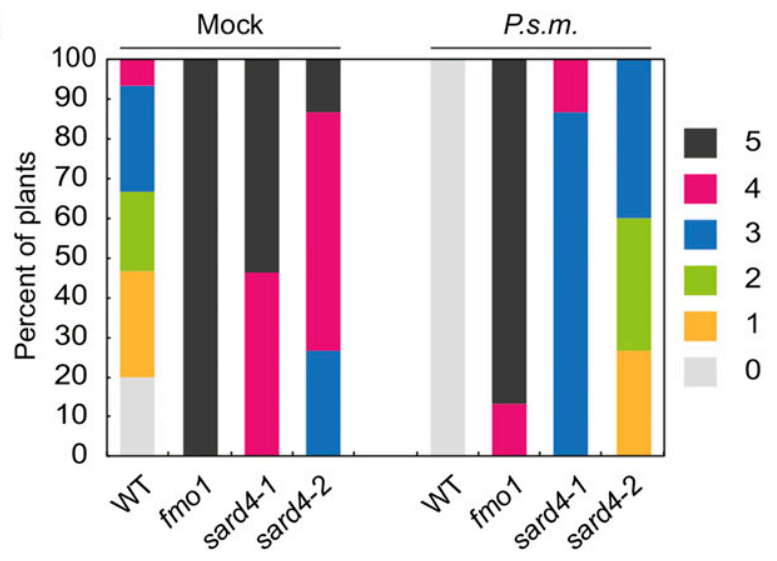

B

C

D
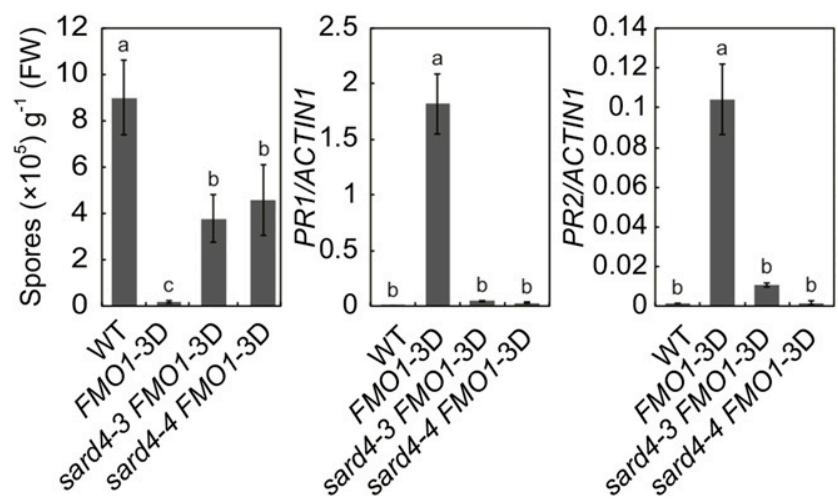

Figure 1. Identification of sard4 Mutant Lines of Arabidopsis.

(A) Growth of H.a. Noco2 on the distal leaves of the wild type, sard4-1, and sard4-2. Three-week-old plants were first infiltrated with P.s.m. ES4326 $\left(\mathrm{OD}_{600}=0.001\right)$ or $10 \mathrm{mM} \mathrm{MgCl}_{2}$ (mock) on two primary leaves and sprayed with H.a. Noco2 spores $\left(5 \times 10^{4}\right.$ spores $\left./ \mathrm{mL}\right) 2$ d later. Infections on systemic leaves were scored $7 \mathrm{~d}$ after inoculation as described previously (Zhang et al., 2010). A total of 15 plants were scored for each treatment. Disease rating scores are as follows: 0 , no conidiophores on the plants; 1 , one leaf was infected with no more than five conidiophores; 2 , one leaf was infected with more than five conidiophores; 3 , two leaves were infected but no more than five conidiophores on each infected leaf; 4 , two leaves were infected with more than five conidiophores on each infected leaf; 5 more than two leaves were infected with more than five conidiophores. Similar results were obtained in three independent experiments.

(B) Growth of H.a. Noco2 on the wild type, FMO1-3D, sard4-3 FMO1-3D, and sard4-4 FMO1-3D. Three-week-old seedlings were sprayed with $H$.a. Noco2 spores $\left(5 \times 10^{4}\right.$ spores $\left./ \mathrm{mL}\right)$. Infection was scored $7 \mathrm{~d}$ after inoculation by counting the numbers of spores per gram of leaf samples. Statistical differences between the samples are labeled with different letters $(P<0.01$, one-way ANOVA; $n=4)$. Similar results were obtained in three independent experiments.

(C) and (D) Expression of $P R 1$ (C) and $P R 2$ (D) in the wild type, FMO1-3D, sard4-3 FMO1-3D, and sard4-4 FMO1-3D. Two-week-old seedlings grown on Murashige and Skoog plates were used for RT-qPCR analysis. Values were obtained from abundances of $P R 1$ and PR2 normalized against that of ACTIN1, respectively. Statistical differences among the samples are labeled with different letters $(P<0.01$, one-way ANOVA; $n=3)$. Similar results were obtained in three independent experiments. 


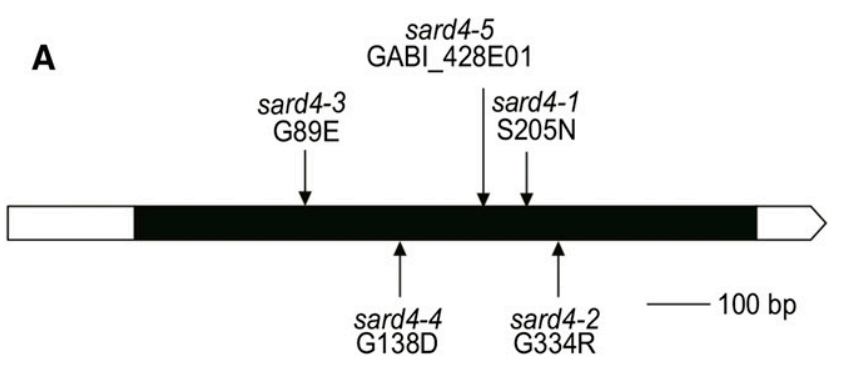

B

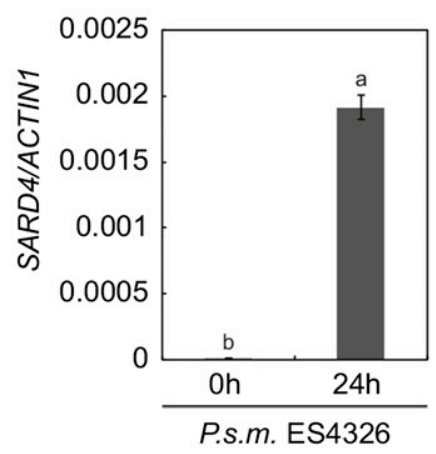

C

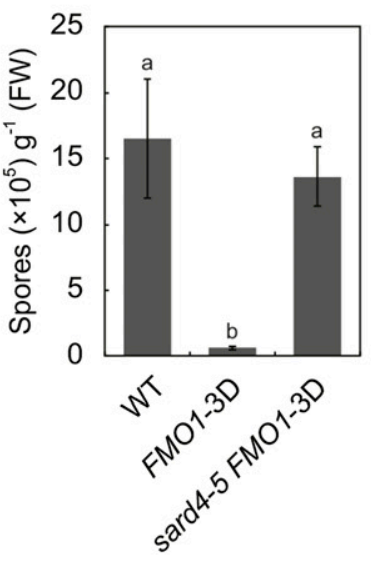

D

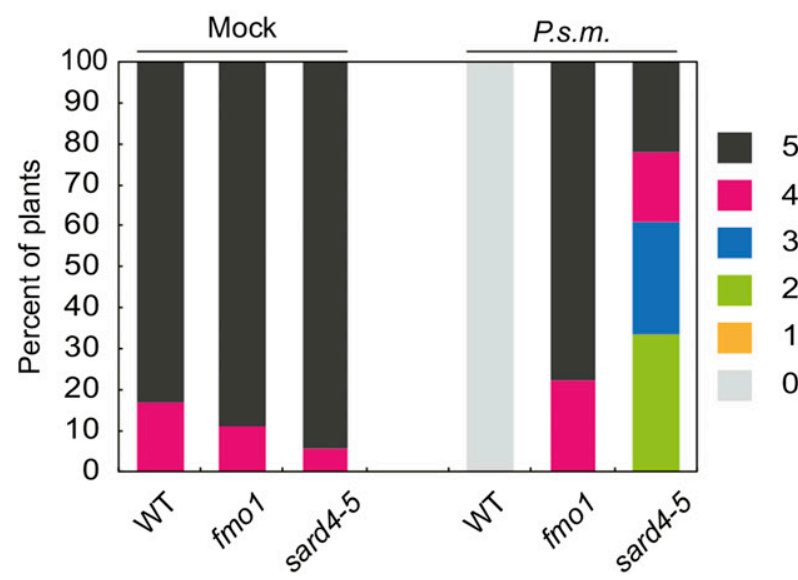

Figure 2. Positional Cloning of SARD4, Expression of SARD4, and the sard4 Phenotype.

(A) Positions of the sard4 mutations in the gene.

(B) Induction of SARD4 transcription by P.s.m. ES4326. Leaves of 3-week old wild-type plants were infiltrated with P.s.m. ES4326 at a dose of $\mathrm{OD}_{600}=$ 0.01 . The inoculated leaves were collected $24 \mathrm{~h}$ later for RT-qPCR analysis. Values were obtained from the abundance of SARD4 transcripts normalized against that of $A C T I N 1$. Statistical differences among the samples are labeled with different letters $(P<0.01$, one-way ANOVA; $n=3)$. Similar results were obtained in three independent experiments.

(C) Growth of H.a. Noco2 on the wild type, FMO1-3D, and sard4-5 FMO13D. Statistical differences between the samples are labeled with different
We also obtained a T-DNA insertion mutant, sard4-5, from ABRC (Figure 2A) and crossed it into FMO1-3D to test whether At5g52810 is required for enhanced pathogen resistance in FMO1-3D. As shown in Figure 2C, the enhanced resistance against H.a. Noco2 in FMO1-3D is lost in the sard4-5 FMO1-3D double mutant. In addition, SAR is also compromised in sard4-5 (Figure 2D). These data confirm that At5g52810 is indeed SARD4 and it is required for SAR as well as enhanced pathogen resistance conferred by overexpression of FMO1.

\section{Systemic Defense Responses Are Compromised in sard4 Plants}

$\mathrm{SA}$ is an important signal molecule required for both local and systemic acquired resistance. The loss of SAR phenotype in sard 4 mutants prompted us to test whether SA accumulation is affected in the mutant plants. As shown in Supplemental Figure 1, P.s.m. ES4326-induced accumulation of SA in local leaves is similar in sard4-5 and wild-type plants. However, induction of SA accumulation in systemic leaves is considerably reduced in sard4-5 (Figure 3A). Consistent with this, induction of PR1 and PR2 expression is reduced in systemic (Figures $3 \mathrm{~B}$ and $3 \mathrm{C}$ ) but not local leaves (Supplemental Figure 2).

To test whether SARD4 is required for resistance against bacteria, we inoculated sard4-5 with P.s.m. ES4326 by infiltration. The npr1-1 mutant was used as a positive control. Growth of bacteria in the local leaves is much higher in npr1-1, but comparable between the wild-type and sard4-5 mutant plants (Supplemental Figure 3). In the systemic leaves of plants pretreated with P.s.m. ES4326, sard4-5 supports significantly higher bacterial growth than the wild type (Figure 3D). These data suggest that SARD4 is required for systemic but not local resistance to $P$.s. m. ES4326.

\section{SARD4 Is Involved in Biosynthesis of Pip}

To identify the substrate for SARD4, we performed metabolite fingerprinting analysis of the systemic tissue of the wild type and sard4-5 after SAR induction. We hypothesized that the substrate of SARD4 should accumulate in the mutant upon infection. The accumulation of Pip was used as an indicator for the establishment of SAR in wild-type plants, using the ald1 (ald1-T2) mutant as a negative control. In this setup, we found 1250 high quality features (false discovery rate $[F D R]<10^{-2}$ ) that showed an altered accumulation pattern. Interestingly, Pip was nearly absent not only in ald1, but also in the sard4-5 mutant (Figure 4A). From these 1250 features, we detected only one that accumulated exclusively in sard4-5 upon infection (Figure 4B). A database query (Kyoto encyclopedia of genes and genomes [KEGG]) based on the accurate mass information acquired by high-resolution mass spectrometry (MS) suggested that the compound was most likely

letters $(P<0.01$, one-way ANOVA; $n=4)$. The experiment was repeated twice with similar results.

(D) Growth of H.a. Noco2 on the distal leaves of the wild type, fmo1, and sard4-5 following mock or P.s.m. ES4326 treatment. The experiment was repeated twice with similar results. 

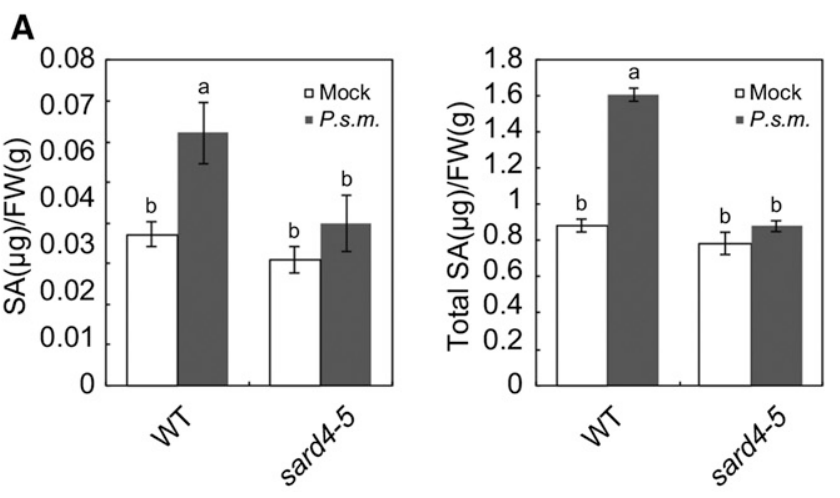

B

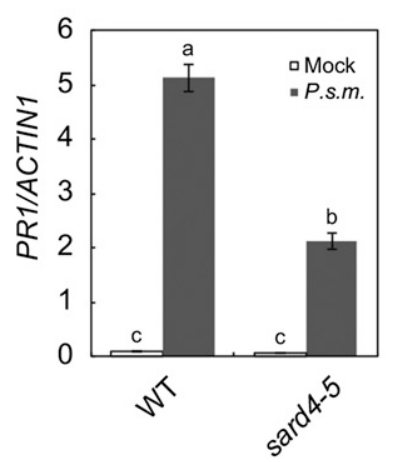

C

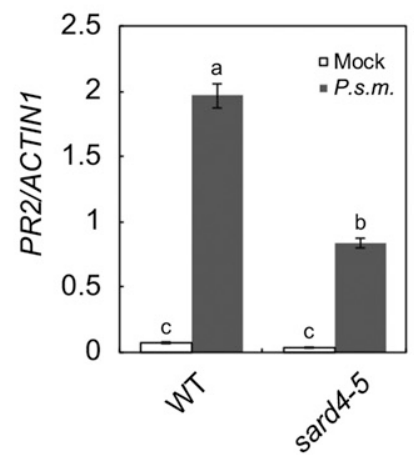

D

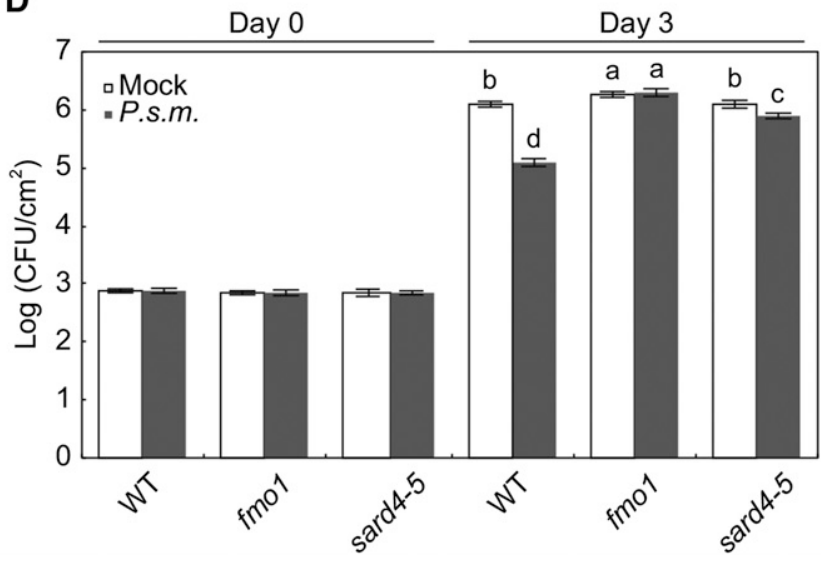

Figure 3. SARD4 Is Required for Systemic Defense Responses.

(A) FreeSA and total SA accumulation in the systemic leaves in the wild type and sard4-5 following local infection by P.s.m. ES4326. Three leaves of 4-week-old plants were infiltrated with P.s.m. ES4326 $\left(\mathrm{OD}_{600}=0.005\right)$, and the distal leaves were collected $48 \mathrm{~h}$ later for SA extraction and quantification. Statistical differences between the samples are labeled with different letters $(P<0.01$, one-way ANOVA; $n=4)$. The experiment was repeated twice with similar results.

(B) and (C) Induction of systemic PR1 (B) and PR2 (C) expression in the wild type and sard $4-5$ by P.s.m. ES4326. Three-week-old plants were infiltrated with P.s.m. ES4326 $\left(\mathrm{OD}_{600}=0.005\right)$ or $10 \mathrm{mM} \mathrm{MgCl}_{2}$ (mock) on two primary leaves, and distal leaves were collected $48 \mathrm{~h}$ later for RT-qPCR analysis. Values were obtained from abundances of $P R 1$ and $P R 2$ transcripts normalized against that of ACTIN1. Statistical differences among the
$\Delta^{1}$-piperideine-2-carboxylic acid (P2C), a catabolite of lysine and a precursor of Pip. The identity of both Pip and P2C were confirmed by MS/MS fragmentation (Figures 4C and 4D). However, lysine may be converted into Pip via two different pathways that can be distinguished by the structures of their intermediates immediately upstream of Pip: $\Delta^{1}$-piperideine-6-carboxylic acid (P6C) and P2C, respectively (Zeier, 2013). The specific formation of P2C was confirmed by spectroscopic and spectrometric methods (see below).

Next, we used gas chromatography-mass spectrometry (GCMS) to analyze Pip levels in systemic tissue of sard4-5 following P.s.m. ES4326 infection. After bacterial treatment, Pip was below detectable levels in ald1 and sard 4 mutants, but accumulated in substantial amounts in wild-type and fmo1 plants (Figure 5A). Using the same analytical method, we also quantified Pip levels in local leaves of sard4-5 inoculated with P.s.m. ES4326. Strong induction of Pip by P.s.m. ES4326 was observed in wild-type, fmo1, and sard4 plants, with a small but significant reduction of Pip induction in sard4-5 (Figure 5B). These data further support the hypothesis that SARD4 is involved in the biosynthesis of Pip.

\section{Reconstitution of the Pip Biosynthesis Pathway by Heterologous Expression of ALD1 and SARD4 in Escherichia coli}

To further analyze the enzymatic activities of SARD4 and ALD1, we introduced Arabidopsis thaliana SARD4 and ALD1 separately and both together into $E$. coli for in-cell activity assays. We hypothesized that internal lysine could be used as a substrate for ALD1 and the resulting product will be further converted to Pip by SARD4. After induction of heterologous expression of both proteins (Supplemental Figure 4), complete cultures were extracted for analysis. In the E. coli culture that expressed ALD1 (Figure 6A), we could detect P2C (dashed line, $\mathrm{m} / \mathrm{z}$ 128.070, RT $0.98 \mathrm{~min}$ ), but no Pip (solid line). In the SARD4-expressing culture (Figure 6B), neither Pip nor P2C could be detected. However, the $E$. coli culture expressing both SARD4 and ALD1 in one strain yielded Pip (Figure 6C, solid line, $\mathrm{m} / \mathrm{z} 130.086$, RT $0.86 \mathrm{~min})$, while in the control containing the empty vectors, neither $\mathrm{Pip}$ nor $\mathrm{P} 2 \mathrm{C}$ was detectable (Figure 6D). Hence, we can exclude that P2C and Pip are E. coliderived metabolites. The structures of both P2C and Pip were confirmed by high-resolution MS/MS analysis (Supplemental Figure 5). The exact mass, retention time, and fragmentation patterns were consistent with those of the compounds obtained from the plant material (Figure 4). Interestingly, $\varepsilon$-amino- $\alpha$-keto

samples are labeled with different letters $(\mathrm{P}<0.01$, one-way ANOVA; $n=3)$. Similar results were obtained in three independent experiments.

(D) Growth of P.s.m. ES4326 on the distal leaves of the wild type, fmo1, and sard4-5.

Three-week-old plants were first infiltrated with P.s.m. ES4326 $\left(\mathrm{OD}_{600}=\right.$ 0.005) or $10 \mathrm{mM} \mathrm{MgCl}_{2}$ (mock) on two primary leaves, and two distal leaves were infected with P.s.m. ES4326 $\left(\mathrm{OD}_{600}=0.0001\right) 2 \mathrm{~d}$ later. Bacterial growth in distal leaves was determined $3 \mathrm{~d}$ after inoculation. Statistical differences between the samples are labeled with different letters $(P<0.01$, one-way ANOVA; $n=6$ ). The experiment was repeated twice with similar results. 

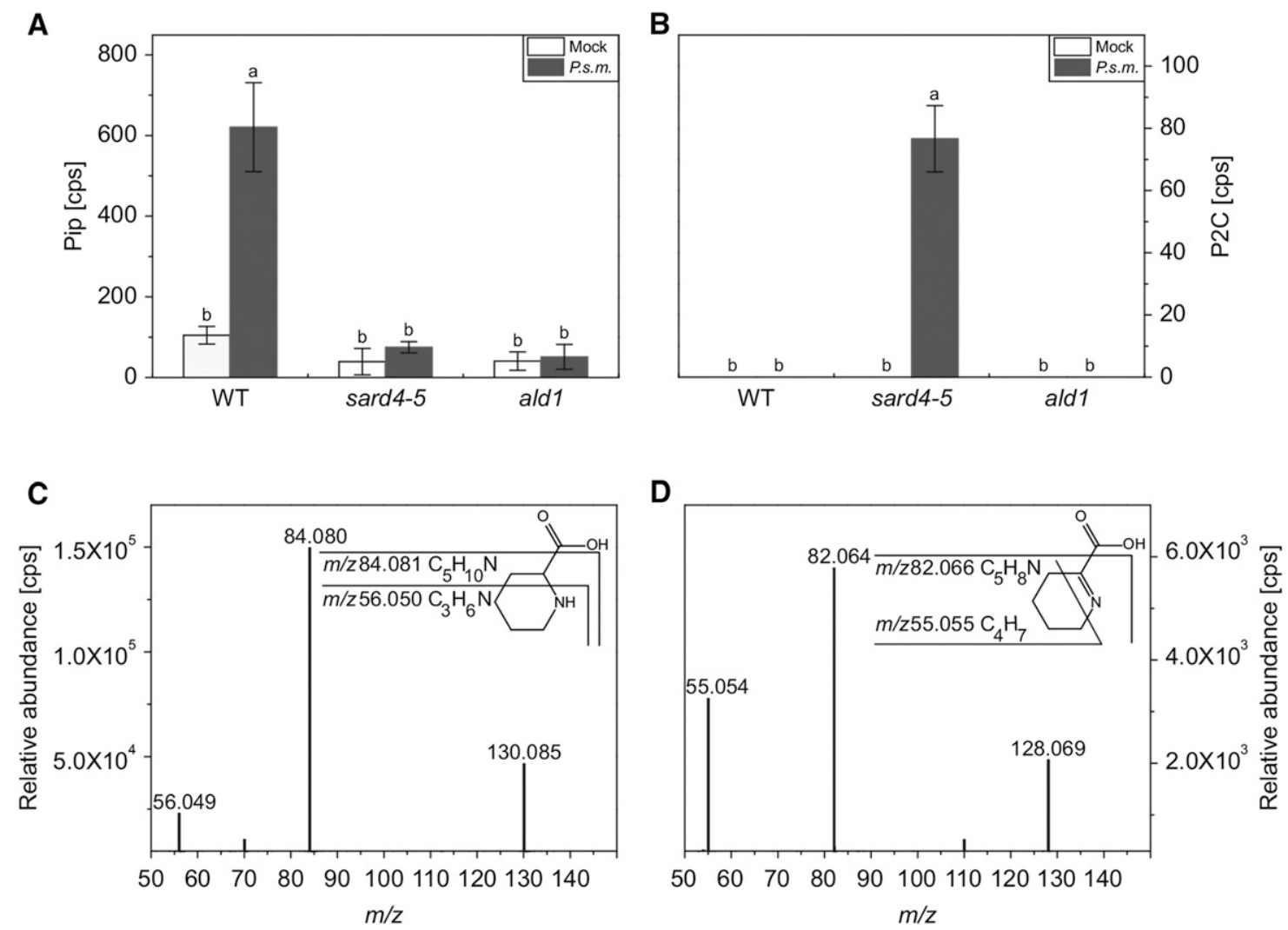

Figure 4. SARD4 Is Involved in Biosynthesis of Pip.

(A) and (B) Relative abundance of Pip and P2C in systemic leaves of Arabidopsis wild type, ald1, and sard4-5 48 h after P.s.m. ES4326 (OD $600=0.005)$ infection. The relative intensities of Pip (A) and P2C (B) are shown. Three biological replicates were analyzed twice by liquid chromatography-mass spectrometry. Statistical differences among the samples are labeled with different letters $(P<0.01$, one-way ANOVA; $n=6)$. The data were obtained from the nontargeted metabolite fingerprint analysis. Similar results were obtained in two independent experiments.

(C) and (D) High-resolution MS/MS fragmentation patterns of Pip and P2C detected in the systemic leaves of P.s.m. ES4326-treated wild-type (C) or sard4-5 mutant plants (D). The fragmentation of Pip (C) and P2C (D) leads to a loss of the carboxyl group $(\mathrm{m} / \mathrm{z} 84.080$ and $\mathrm{m} / \mathrm{z} 82.065$, respectively). In Pip, the mass signal of $m / z 56.049$ represents a $\mathrm{C}_{3} \mathrm{H}_{6} \mathrm{~N}$-fragment. In P2C, the $\mathrm{C}=\mathrm{N}$ double bond stays intact so that the mass signal of $m / z 55.054$ represents a $\mathrm{C}_{4} \mathrm{H}_{7}-$ fragment.

caproic acid (Figure 6E), the proposed product of ALD1 (Zeier, 2013), was nearly undetectable in the samples from the in-cell activity assay. Most likely, it was completely converted into P2C under the applied conditions.

To summarize, we could identify P2C as a product of ALD1 and a substrate of SARD4, and Pip as a product of SARD4. These findings allow us to reconstruct one of the plant Pip biosynthesis pathways in E. coli (Figure 6E). As we did not detect accumulation of other metabolites in this in-cell assay upon expression of either SARD4 or ALD1 alone or both together, the data suggest that the proposed pathway may exclusively produce Pip using lysine as substrate.

\section{Unequivocal Identification of P2C}

To further study the reaction of ALD1, we followed a previously published protocol to purify the heterologously expressed ALD1 (Sobolev et al., 2013). The homogenous enzyme (Supplemental Figure 6) was first tested for its in vitro activity with L-lysine to determine whether ALD1 produces P2C or P6C. These compounds can be distinguished by their different absorbance maxima when derivatized with o-aminobenzaldehyde (Soda et al., 1968). We treated the product of the ALD1/L-lysine reaction with $o$-aminobenzaldehyde and the formed reaction product showed an absorbance maximum at $446 \mathrm{~nm}$ (Figure 7A). This maximum corresponds with the reaction product of P2C (Soda et al., 1968) and confirms P2C as the product of ALD1. Next, we incubated ALD1 with L-lysine and L-lysine- $6-{ }^{13} \mathrm{C},-{ }^{15} \mathrm{~N}$ separately and analyzed the products of the reactions by high resolution MS/MS. The fragmentation pattern of the L-lysine-derived product was highly similar to that of the compounds from both the leaf material (Figure 4 ) and the in-cell assay (Supplemental Figure 5). When we used L-lysine- $6-{ }^{13} \mathrm{C},-{ }^{15} \mathrm{~N}$ as the substrate for ALD1, we detected the mass signal of $m / z 130.0714$ as base peak at $0.97 \mathrm{~min}$, which represents the molecular ion of $6-{ }^{13} \mathrm{C}-{ }^{15} \mathrm{~N}$-labeled $\mathrm{P} 2 \mathrm{C}$ in the positive ionization mode (Figure 7B). The mass deviation between the calculated exact mass of positively charged ion of $6-{ }^{13} \mathrm{C}-1{ }^{15} \mathrm{~N}$-labeled P2C ( $m / z 130.0715)$ and the accurate mass of the molecular ion 

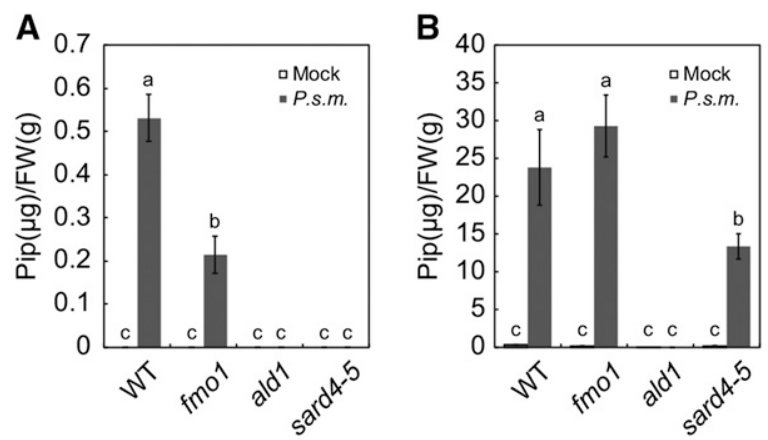

Figure 5. Pip Levels in Wild-Type, fmo1, ald1, and sard4-5 Plants.

(A) Pip levels in distal tissue of the wild type, fmo1, ald1, and sard4-5 following infection by P.s.m. ES4326. Primary leaves were infiltrated with a bacterial suspension of P.s.m. $\mathrm{ES} 426$ at $\mathrm{OD}_{600}=0.005$. The distal leaves were collected $48 \mathrm{~h}$ later for amino acid analysis.

(B) Pip levels in local tissue of the wild type, fmo1, ald1, and sard4-5 following infection by P.s.m. ES4326. The inoculated leaves were collected for amino acid analysis $48 \mathrm{~h}$ after inoculation with the bacteria $\left(\mathrm{OD}_{600}=\right.$ 0.005).

Statistical differences between the samples are labeled with different letters $(P<0.01$, one-way ANOVA; $n=3$ ). Similar results were obtained in two independent experiments.

derived by our high-resolution MS analysis (see above) was $0.1 \mathrm{mD}$. The high-resolution MS/MS revealed the presence of both isotopes in the fragment of $m / z 84.0658$ (loss of the carboxyl group). The mass signal of $m / z 56.0578$ represents the $\mathrm{C}_{3}{ }^{13} \mathrm{CH}_{7}$ fragment, containing only the labeled carbon. This fragmentation pattern corresponds to fragmentation of the unlabeled P2C from the leaf material (Figure 4D). As the photometric assay and the high-resolution MS analysis confirmed $\mathrm{P} 2 \mathrm{C}$ as the product of ALD1, we concluded that ALD1 is an $\alpha$-aminotransferase that can use L-lysine for the formation of P2C.

\section{SARD4 Converts P2C to Pip in Vitro}

To assay the activity of SARD4, the protein was heterologously expressed in E. coli and purified to homogeneity (Supplemental Figure 7). As P2C is not commercially available, we used $\Delta 20$ ALD1 (atruncated ALD1) to generate P2C from L-lysine and $6-{ }^{13} \mathrm{C}-,{ }^{15} \mathrm{~N}-$ labeled P2C from L-lysine- $6-{ }^{13} \mathrm{C},-{ }^{15} \mathrm{~N}$ (see section above). Filter centrifugation was employed to remove $\triangle 20$-ALD1 from the product solution. As shown in Figure 8A, labeled P2C (dashed line, $\mathrm{m} / \mathrm{z}$ 130.072, RT $0.97 \mathrm{~min}$ ) but no labeled Pip (solid line) was present in the $\Delta 20$-ALD1-free product solution. The addition of purified SARD4 protein to this solution led to a complete conversion of labeled P2C to labeled Pip (Figure 8B, solid line; $m / z$ 132.086, RT $0.87 \mathrm{~min}$ ). To verify that the product was indeed Pip, we compared the retention time and MS/MS fragmentation pattern of commercial Pip standard with the SARD4 produced in vitro product (Figures $8 \mathrm{C}$ and $8 \mathrm{D}$ ). Analogous to the unlabeled Pip, the fragmentation leads to a loss of the carboxyl group $(\mathrm{m} / \mathrm{z}$ 86.082), in which both isotopes are still present. The mass signal of $\mathrm{m} / \mathrm{z}$ 58.050 represents a $\mathrm{C}_{2}{ }^{13} \mathrm{CH}_{6}{ }^{15} \mathrm{~N}$ fragment, which contains both the ${ }^{13} \mathrm{C}$ and the ${ }^{15} \mathrm{~N}$ isotope. The retention time and the fragmentation pattern were identical for the SARD4-derived in vitro product, the compound identified in the leaf material (Figure 4), the in-cell assay (Figure 6; Supplemental Figure 5), and the commercial Pip standard. Together, these data strongly suggest that SARD4 catalyzes the reduction of P2C to Pip.

\section{Pip Restores PR Gene Expression in sard4-4 FM01-3D}

To test whether exogenous application of Pip can complement the defects in sard4-4, we grew sard4-4 FMO1-3D on media containing Pip and measured the expression levels of $P R$ genes. As shown in Figures 9A and 9B, the expression of $P R 1$ and $P R 2$ in sard4-4 FMO1-3D seedlings was largely restored by Pip, indicating that treatment with Pip complements the defects in Pip biosynthesis in sard4 mutant plants.

\section{ALD1 Is Required for Enhanced Defense Responses in FMO1-3D}

As Pip biosynthesis is blocked in ald1 mutant plants (Návarová et al., 2012), we tested whether ALD1 is also required for constitutive defense responses in FMO1-3D. We crossed ald1 into FMO1-3D and analyzed $P R$ gene expression and resistance to H.a. Noco2 in ald1 FMO1-3D double homozygous plants. As shown in Figures $10 \mathrm{~A}$ and 10B, constitutive expression of $P R 1$ and $P R 2$ in FMO1-3D is blocked in the double mutant. In addition, enhanced resistance to H.a. Noco2 in FM01-3D is also abolished by ald1 (Figure 10C), which is consistent with loss of resistance to H.a. Noco2 in the sard4 FMO1-3D double mutant.

\section{DISCUSSION}

Pip has been shown to play important roles in orchestrating plant defense responses (Návarová et al., 2012) and SAR is abolished in the Pip-deficient mutant ald1 (Song et al., 2004b). A recent study had suggested that ALD1 is also involved in production of non-Pip metabolites critical for the induction of plant immunity (Cecchini et al., 2015). To what extent lack of Pip contributes to the SAR deficiency in ald1 mutant plants is unclear. In this study, we identified SARD4 as a critical enzyme involved in Pip biosynthesis. Compromised SAR in the sard 4 mutants provides clear evidence that Pip is required for the establishment of SAR.

Analysis of local defense responses against P.s.m. ES4326 showed that there is no significant difference in $P R$ gene expression and SA accumulation between wild-type and sard4 mutant plants, suggesting that Pip produced by SARD4 has little contribution to local resistance against P.s.m. ES4326. This is consistent with the relatively small effect of the sard4-5 mutation on pathogen-induced Pip accumulation in local tissue. Interestingly, mock-treated sard4-1 and sard4-2 mutants displayed enhanced susceptibility to H.a. Noco2, suggesting that SARD4 may play a role in basal resistance against the oomycete pathogen.

SARD4 shares sequence similarity with bacterial ornithine cyclodeaminases, but it does not show the corresponding enzyme activity, consistent with the observation that the three residues critical for activity of bacterial ornithine cyclodeaminases are not 

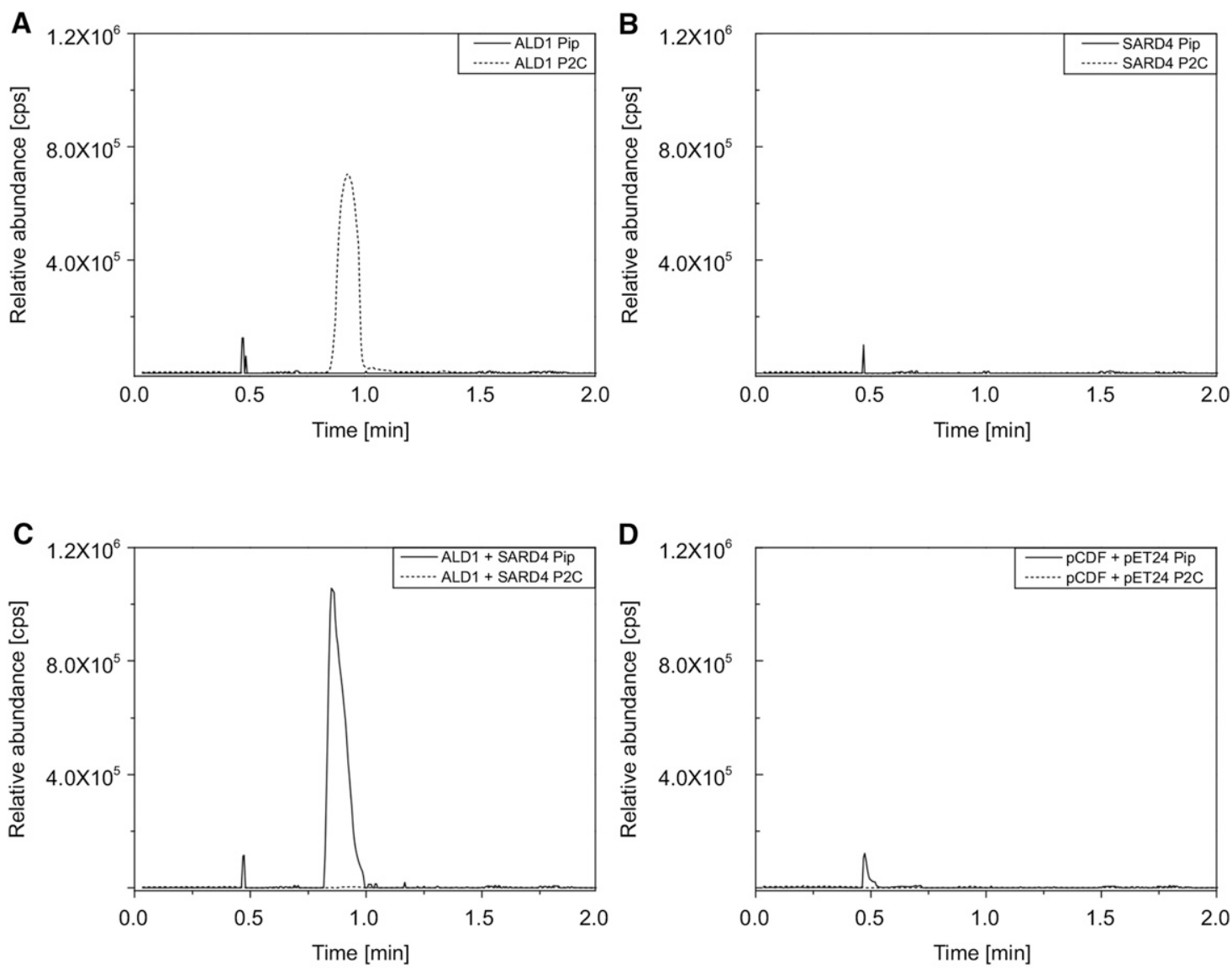

E

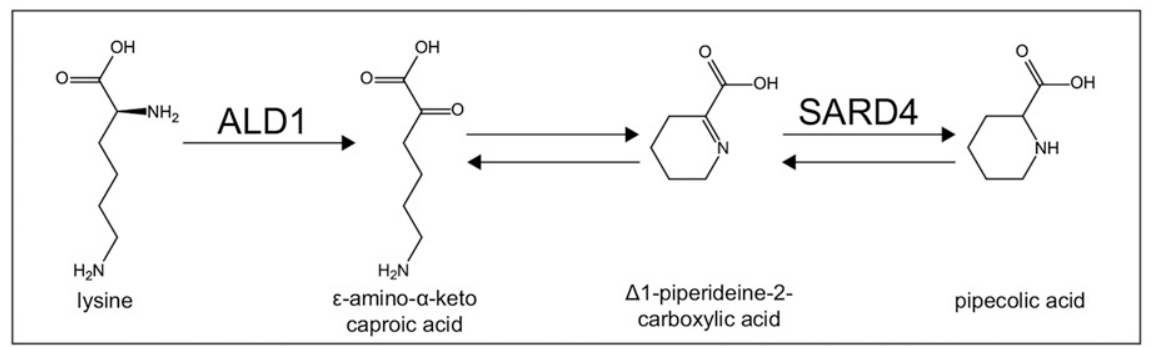

Figure 6. Analysis of the Enzymatic Activity of ALD1 and SARD4 in E. coli.

(A) to (D) Extracted ion chromatograms for P2C ( $\mathrm{m} / \mathrm{z}$ 128.070, dotted lines) and Pip ( $\mathrm{m} / \mathrm{z} 130.086$, solid lines). For the analysis, E. coli cultures expressing ALD1 (A), SARD4 (B), ALD1 and SARD4 together (C), or the two corresponding empty vectors (D) were used. Similar results were obtained in two independent experiments.

(E) Proposed scheme for the Pip biosynthesis pathway from lysine.

conserved in SARD4 (Sharma et al., 2013). Multiple lines of evidence suggest that SARD4 functions as a reductase that converts $\mathrm{P} 2 \mathrm{C}$ to Pip. sard 4 mutant plants not only have reduced Pip levels, they also accumulate $\mathrm{P} 2 \mathrm{C}$, a proposed Pip precursor, in distal leaves following infection with P.s.m. ES4326 (Figure 4). In addition, P2C is converted to Pip in E. coli when SARD4 is expressed and it can also be converted to Pip in vitro by purified SARD4 protein (Figures 6 and 8).
ALD1 was predicted to use lysine as a substrate and catalyze its conversion to $\varepsilon$-amino- $\alpha$-keto caproic acid, which then could spontaneously cyclize to form P2C (Song et al., 2004a; Zeier, 2013). In E. coli expressing ALD1, we detected high levels of P2C but no $\varepsilon$-amino- $\alpha$-keto caproic acid, suggesting that $\varepsilon$-amino$\alpha$-keto caproic acid does indeed spontaneously cyclize to form P2C. We further tested whether addition of lysine to the media leads to increased production of P2C. Interestingly, supplementation of 
A

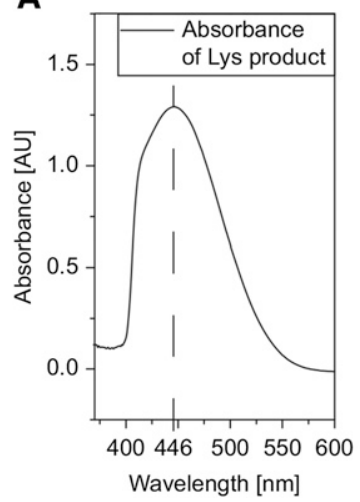

B

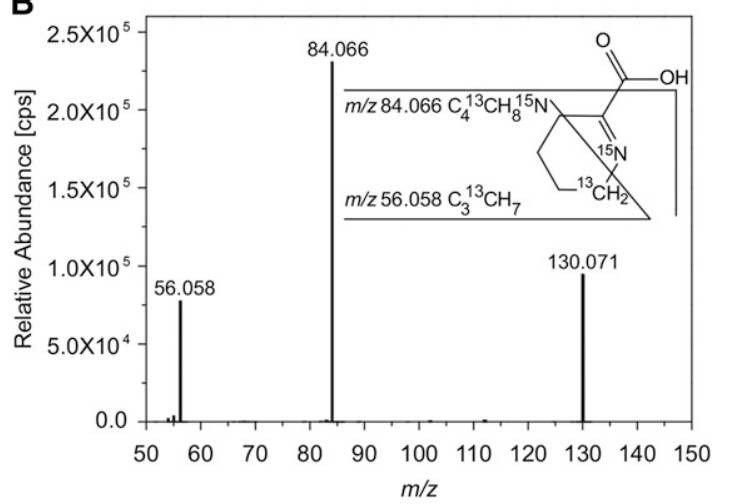

Figure 7. Identification of $\mathrm{P} 2 \mathrm{C}$ as the ALD1 Reaction Product.

(A) Absorption spectrum (background corrected) of the ALD1 reaction product after treatment with o-AB. To remove ALD1 from the solution, filter centrifugation was used. One hundred microliters of the sample was incubated with $890 \mu \mathrm{L}$ sodium acetate buffer $(0.2 \mathrm{M}, \mathrm{pH} 5)$ and $10 \mu \mathrm{L} O-A B(0.4 \mathrm{M})$ for $1 \mathrm{~h}$ at $37^{\circ} \mathrm{C}$. Similar results were obtained in two independent experiments.

(B) MS/MS fragmentation pattern of $6-{ }^{13} \mathrm{C}-,{ }^{15} \mathrm{~N}$-labeled P2C $(\mathrm{m} / \mathrm{z} 130.070)$ from the $\Delta 20$-ALD1 reaction with $\mathrm{L}-\mathrm{lysine}-6-{ }^{13} \mathrm{C},-{ }^{15} \mathrm{~N}$ as substrate. Analogous to the unlabeled P2C, fragmentation leads to a loss of the carboxyl group. In the corresponding fragment $(\mathrm{m} / \mathrm{z} 84.066)$ both isotopes are still present. The mass signal of $\mathrm{m} / \mathrm{z} 56.058$ represents $\mathrm{C}_{3}{ }^{13} \mathrm{CH}_{7}$ fragment, containing the labeled carbon only. Consistent fragmentation pattern of the MS/MS spectra was obtained with $6-{ }^{13} \mathrm{C}-{ }^{15} \mathrm{~N}$-labeled P2C as well as with unlabeled P2C.

lysine did not affect P2C levels (data not shown), suggesting that lysine abundance did not limit P2C production in the bacterial cells. When ALD1 and SARD4 are coexpressed in E. coli, Pip accumulates to high levels and almost no P2C was detected, suggesting that $\mathrm{P} 2 \mathrm{C}$ is an intermediate in Pip biosynthesis and that it was entirely converted into Pip by SARD4 in the bacterial culture. Reconstitution of the Pip biosynthesis pathway in E. coli strongly supports the hypothesis that Pip is synthesized through reduction of $\mathrm{P} 2 \mathrm{C}$ by SARD4.

In local tissue, a small reduction of the Pip level was observed in sard4 compared with wild-type plants and there is still high level of Pip accumulation following infection by P.s.m. ES4326, suggesting that loss of function of SARD4 does not completely block Pip synthesis in local tissue. Because SARD4 is a single copy gene in Arabidopsis, this is probably not caused by simple functional redundancy. It is likely that $\mathrm{P} 2 \mathrm{C}$ can be converted to $\mathrm{Pip}$ by another unrelated dehydrogenase, which is only induced in local tissue. However, we cannot rule out the possibility that Pip is also synthesized in local tissue through an intermediate other than P2C. It will be interesting to determine whether ALD1 is involved in production of metabolites other than $\mathrm{P} 2 \mathrm{C}$ and whether SARD4 can use other metabolites as substrates in Arabidopsis.

Despite that Pip accumulates to very high level in the local leaves of sard4 following P.s.m. ES4326 infection, very little Pip is present in systemic leaves, suggesting that there is no significant amount of Pip transported from local leaves to the distal leaves in the mutant. Lack of Pip accumulation in systemic leaves of sard4 indicates that Pip biosynthesis in systemic tissue is SARD4dependent and proceeds primarily via $\mathrm{P} 2 \mathrm{C}$. It also suggests that SARD4-mediated Pip biosynthesis in systemic tissue plays a critical role in establishing SAR.

In plants, pathogen-induced SA synthesis occurs in plastids (Garcion et al., 2008), but where Pip is made in the plant cell is unclear. ALD1 was predicted to be a plastid-localized protein and had been shown to localize to the chloroplast (Cecchini et al., 2015). Similarly, SARD4 was also shown to be a chloroplastlocalized protein (Sharma et al., 2013). The localization of ALD1 and SARD4 suggests that Pip is also synthesized in the plastids. Whether Pip exerts its function in the plastids or is translocated to other parts of the cell to promote plant immunity remains to be determined.

FMO1 encodes a monooxygenase that is required for both local resistance and SAR (Bartsch et al., 2006; Koch et al., 2006; Mishina and Zeier, 2006). The mechanism by which FMO1 regulates plant immunity is unknown. Our study provided clear genetic evidence that Pip is required for activation of defense responses by FMO1. Not only constitutive defense responses in plants overexpressing FMO1 require both ALD1 and SARD4, treatment of Pip also restores $P R$ gene expression in the sard4-4 FM01-3D double mutant. Previously it was shown that fmo1 mutant plants accumulate higher levels of Pip than the wild type (Návarová et al., 2012). It is likely that FMO1 is involved in the synthesis of a defense signal molecule derived from Pip. Identification of the metabolite produced by FMO1 will further advance our understanding of how SAR is established.

\section{METHODS}

\section{Plant Material and Growth Conditions}

sard4-1 and sard4-2 were identified from a previously described forward genetic screen for SAR-deficient mutants (Jing et al., 2011). The FMO1overexpressing mutant FMO1-3D in Arabidopsis thaliana Col-0 background (Koch et al., 2006) and rpp5 mutant in Landsberg erecta (Ler) background were described previously (Parker et al., 1997). sard4-3 FMO13D and sard4-4 FMO1-3D were identified from an EMS-mutagenized population of FMO1-3D by looking for plants that are susceptible to 

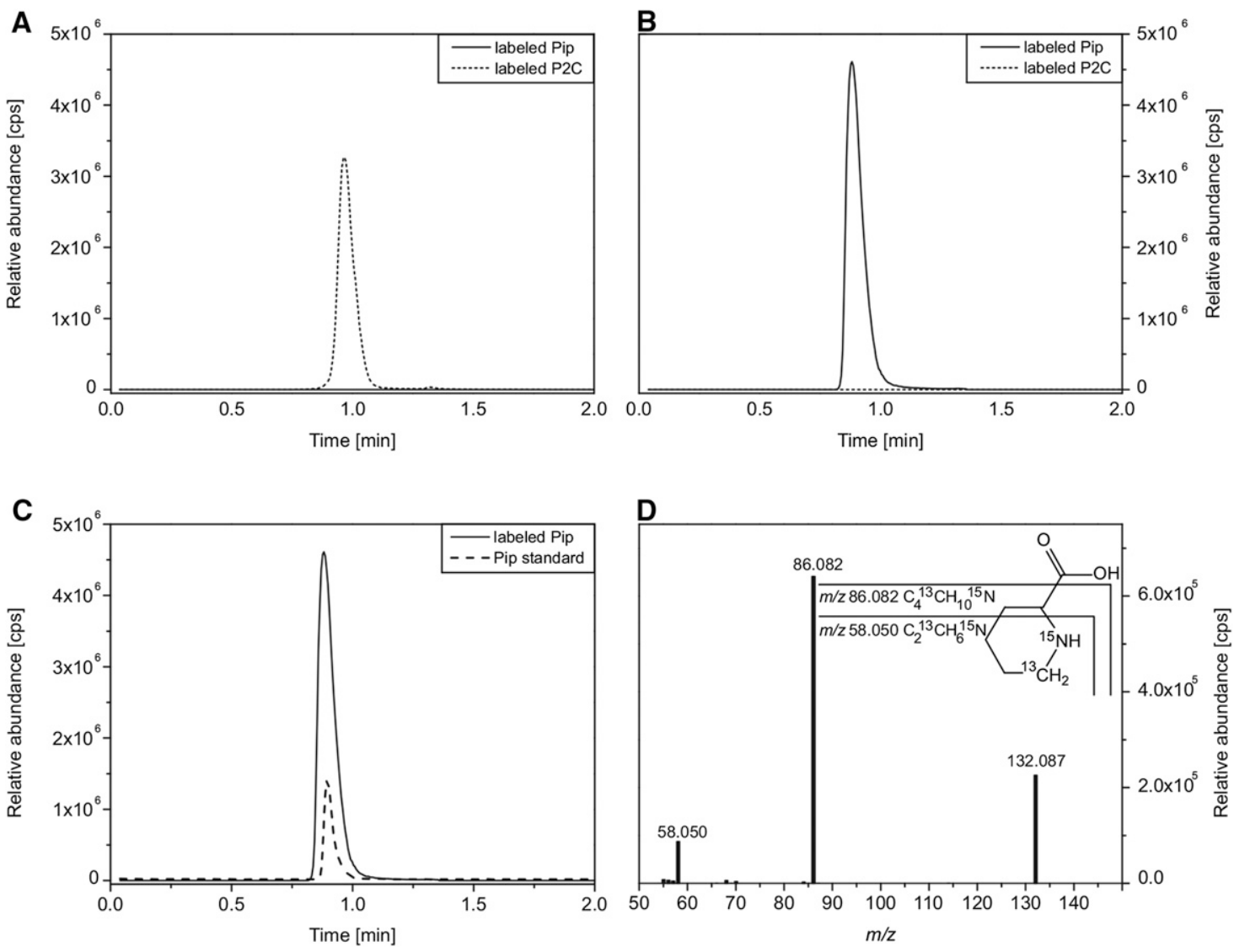

Figure 8. SARD4 Can Convert P2C into Pip in Vitro.

(A) to (C) UHPLC-QTOF-MS analyses of substrates and products of the SARD4 catalyzed reaction. Extracted ion chromatograms of 6-13 C-, ${ }^{15} \mathrm{~N}$-labeled $\Delta^{1}$ piperideine-2-carboxylic (labeled P2C, $\mathrm{m} / \mathrm{z}$ 130.070, dotted lines) (A), $6-{ }^{13} \mathrm{C}-,{ }^{15} \mathrm{~N}$-labeled pipecolic acid (labeled Pip, $\mathrm{m} / \mathrm{z}$ 132.086, solid lines) (B), and pipecolic acid standard (Pip, $\mathrm{m} / \mathrm{z}$ 130.086, dashed line) (C). $6-{ }^{13} \mathrm{C}-{ }^{15} \mathrm{~N}$-labeled Pip and commercial Pip standard show the same retention time. (D) MS/MS fragmentation pattern of $6-{ }^{13} \mathrm{C}-,{ }^{15} \mathrm{~N}$-labeled Pip from the SARD4 reaction. $6-{ }^{13} \mathrm{C}-{ }^{15} \mathrm{~N}$-labeled P2C was used as the substrate, which was produced from L-lysine- $6-{ }^{13} \mathrm{C},-{ }^{15} \mathrm{~N}$ by the $\Delta 20$-ALD1 reaction. Analogous to the unlabeled Pip, fragmentation leads to a loss of the carboxyl group. In the corresponding fragment $(\mathrm{m} / \mathrm{z} 86.082)$, the ${ }^{13} \mathrm{C}$ as well as the ${ }^{15} \mathrm{~N}$ isotopes are still present. The mass signal of $m / z 58.050$ represents a $\mathrm{C}_{2}{ }^{13} \mathrm{CH}_{6}{ }^{15} \mathrm{~N}$ fragment, still containing both isotopes. Consistent results were obtained with $6-{ }^{13} \mathrm{C}-,{ }^{15} \mathrm{~N}$-labeled P2C as well as with unlabeled P2C.

H.a. Noco2. sard4-5 (GABI_428E01) was obtained from the ABRC. ald1-T2 (SALK_007673) was described previously (Song et al., 2004a). PCR primers used for genotyping sard4-5 and ald1-T2 mutants are listed in Supplemental Table 1. Plants were grown under $16 \mathrm{~h}$ white light (Sylvania Octron ${ }^{\mathrm{R}} 4100 \mathrm{~K}, \mathrm{FO} 32 / 741 / \mathrm{ECO}$ bulbs) at $23^{\circ} \mathrm{C} / 8 \mathrm{~h}$ dark at $19^{\circ} \mathrm{C}$ in a plant growth room unless specified.

\section{Mutant Characterization}

Analysis of resistance to H.a. Noco2 in FMO1-3D was performed by spraying 2-week-old seedlings with H.a. Noco2 spores at a concentration of $5 \times 10^{4}$ spores $/ \mathrm{mL}$. Growth of H.a. Noco2 was quantified $7 \mathrm{~d}$ later as previously described (Bi et al., 2010). Bacterial infection assays for testing local resistance were performed by infiltrating two full-grown leaves of 4-week-old plants grown under short-day conditions $\left(12 \mathrm{~h}\right.$ light at $23^{\circ} \mathrm{C} /$ $12 \mathrm{~h}$ dark at $19^{\circ} \mathrm{C}$ ). Bacterial growth was assessed $3 \mathrm{~d}$ after inoculation. Induction of SAR against H.a. Noco2 was performed as previously described (Zhang et al., 2010) by infiltrating two primary leaves of 3-weekold plants with P.s.m. ES4326 $\left(\mathrm{OD}_{600}=0.001\right)$. The plants were sprayed with H.a. Noco2 spores at a concentration of $5 \times 10^{4}$ spores $/ \mathrm{mL} 2 \mathrm{~d}$ later.
Induction of SAR against P.s.m. ES4326 was performed by infiltrating two primary leaves of 4-week-old plants with P.s.m. ES4326 $\left(\mathrm{OD}_{600}=0.005\right)$. Two distal leaves were infiltrated with the same bacteria $\left(\mathrm{OD}_{600}=0.0001\right)$ $2 \mathrm{~d}$ later to assess SAR.

Absolute quantification of Pip was done using the EZ:faast free amino acid analysis kit for GC-MS (Phenomenex), which is based on GC separation and mass spectrometric identification and quantification of propyl chloroformate-derivatized amino acids. Each GC sample was prepared by extracting $50 \mathrm{mg}$ leaf tissue and analyzed following a procedure described previously (Návarová et al., 2012).Three biological replicates were analyzed in each experiment. SA was extracted from four biological replicates with each consisting of $\sim 100 \mathrm{mg}$ of tissue in each experiment and quantified by HPLC as previously described (Sun et al., 2015).

For gene expression analysis, RNA was isolated from three biological replicates and used for subsequent RT-qPCR analysis. Briefly, RNA was extracted using the EZ-10 Spin Column Plant RNA Mini-Preps Kit from Biobasic (Canada) and treated with RQ1 RNase-Free DNase (Promega) to remove the genomic DNA contamination. Reverse transcription was performed using the EasyScript Reverse Transcriptase (ABM). QPCR was performed using the Takara SYBR Premix Ex (Clontech). Primers for qPCR 
A
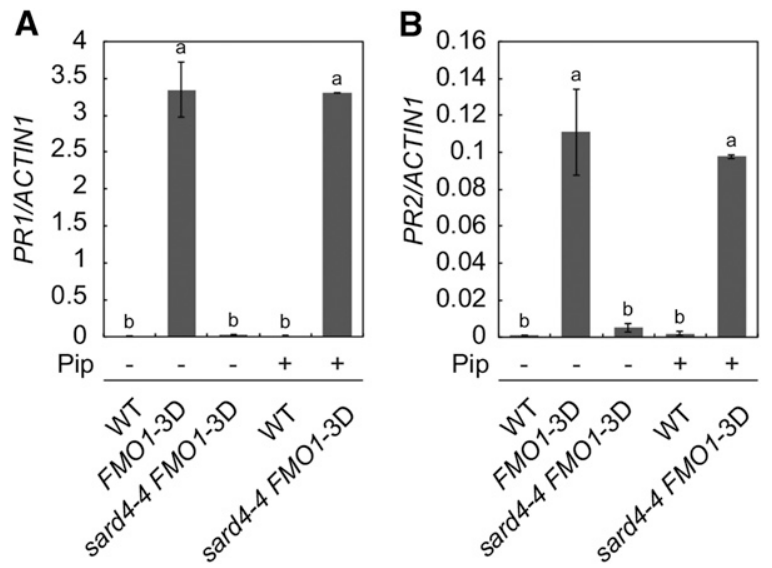

Figure 9. Pip Restores $P R 1$ and $P R 2$ Expression in sard4-4 FMO1-3D.

Two-week old seedlings of the wild type, FMO1-3D, and sard4-4 FMO1-3D grown on Murashige and Skoog plates with or without Pip $(5 \mu \mathrm{M})$ were used for RT-qPCR analysis. Values were obtained from the abundance of $P R 1$ and $P R 2$ transcripts normalized against that of ACTIN1, respectively. Statistical differences among the samples are labeled with different letters $(P<0.01$, one-way ANOVA; $n=3)$. Similar results were obtained in three independent experiments.

analysis of SARD4 are listed in Supplemental Table 1. Primers for qPCR analysis of $P R 1, P R 2$, and $A C T I N 1$ were described previously (Zhang et al., 2003).

\section{Genetic Mapping of sard4 Mutants}

To map the sard4-3 and sard4-4 mutations, sard4-3 FMO1-3D and sard4-4 FMO1-3D in Col-0 ecotype background was crossed with an rpp5 mutant in the Ler background to generate segregating mapping populations. The rpp5 mutant was used because RPP5 confers resistance against H.a. Noco2 in Ler. In the F2 population, plants containing the FMO1-3D mutation were selected by their resistance to Basta and assayed for resistance against H.a. Noco2. Plants susceptible to H.a. Noco2 were used for subsequent mapping analysis, which was performed as previously described (Zhang et al., 2007). The primer sequences of the Indel markers are listed in Supplemental Table 1.

\section{Metabolite Fingerprinting}

For the nontargeted metabolite analysis, $100 \mathrm{mg}$ leaf material (three biological replicates per condition) was extracted using two-phase extraction with methy-tert-butylether (Bruckhoff et al., 2016). The polar phase was evaporated, mixed with $100 \mu \mathrm{L}$ methanol, shaken for $5 \mathrm{~min}$, and then centrifuged for $5 \mathrm{~min}$ at $16,000 \mathrm{~g}$ at room temperature. Samples were dried carefully under a nitrogen stream and resuspended in $15 \mu \mathrm{L}$ methanol and shaken for $10 \mathrm{~min}$. Fifteen microliters of acetonitrile was added followed by $10 \mathrm{~min}$ of shaking. Lastly, $100 \mu \mathrm{L}$ of deionized water was added and shaken for $10 \mathrm{~min}$. The samples were centrifuged for $10 \mathrm{~min}$ at $16,000 \mathrm{~g}$ at room temperature and transferred into glass vials. All samples were stored at $4^{\circ} \mathrm{C}$.

Metabolic fingerprinting was performed by UPLC coupled to a time-offlight mass spectrometer (TOF-MS) and a photodiode array as previously described with minor modifications (König et al., 2012). The samples of the polar extraction phase were separated with an Acquity UPLC HSS T3 column $(1.0 \times 100 \mathrm{~mm}, 1.8-\mu \mathrm{m}$ particle size; Waters $)$ at $40^{\circ} \mathrm{C}$ and a flow rate of $0.2 \mathrm{~mL} / \mathrm{min}$. The binary gradient consisted of solvent $A$ (ultrapure water) and solvent $B$ (acetonitrile), each with $0.1 \%(\mathrm{v} / \mathrm{v})$ formic acid. The gradient setup for the polar phase samples was as follows: 0 to $0.5 \mathrm{~min} 99 \% \mathrm{~A}, 0.5$ to $3 \mathrm{~min} 80 \% \mathrm{~A}, 3$ to $8 \mathrm{~min}$ from $20 \%$ to $100 \% \mathrm{~B}, 10$ to $10.1 \mathrm{~min} 100 \% \mathrm{~B}, 10.1$ to $14 \min 99 \%$ A.

All samples were measured twice by TOF-MS in both positive and negative electrospray ionization (ESI) mode with dynamic range enhancement. In the positive ESI mode, mass range from $\mathrm{m} / \mathrm{z} 85.00$ to $\mathrm{m} / \mathrm{z}$ 1200 was detected, whereas in the negative ESI mode, mass range from $\mathrm{m} / \mathrm{z} 50.00$ to $\mathrm{m} / \mathrm{z} 1200$ was used. MS setup was described earlier (König et al., 2012). For the analysis of the raw mass data, the samples were processed (peak picking and peak alignment) using the MarkerLynx Application Manager 4.1 for MassLynx software, which resulted in two data matrixes. For further data processing, ranking, filtering, adduct correction, clustering, and database analysis, MarVis software (MarkerVisualization) (Kaever et al., 2015) was used. For ranking and filtering of the data sets in MarVis Filter, an ANOVA test combined with adjustment for multiple testing by Benjamini-Hochberg algorithm (FDR) was applied. The threshold was set at FDR $<0.01$. The subsets of high-quality features were adductcorrected according to the following rules: $[\mathrm{M}+\mathrm{H}]^{+},[\mathrm{M}+\mathrm{Na}]^{+},\left[\mathrm{M}+\mathrm{NH}_{4}\right]^{+}$(for data of the positive ESI mode); $[\mathrm{M}-\mathrm{H}]^{-},\left[\mathrm{M}+\mathrm{CH}_{2} \mathrm{O}_{2}-\mathrm{H}\right]^{-},\left[\mathrm{M}+\mathrm{CH}_{2} \mathrm{O}_{2}+\mathrm{Na}-\right.$ $2 \mathrm{H}^{-}$(for data of the negative ESI mode) and subsequently combined. The resulting 1250 features were clustered according to similarities in the intensity profiles by means of one-dimensional self-organizing maps and visualized as heat map representation (MarVis Cluster). The accurate mass information of features of interest was used to search databases MetaCyc (http://www.arabidopsis.org/biocyc/) and KEGG (http://www.genome.jp/ kegg/) for putative identities (MarVis Pathway). A mass window of $0.007 \mathrm{D}$ was applied.

\section{High-Resolution MS/MS Analysis (UHPLC-Q-TOF-MS)}

To confirm the chemical structure of marker metabolites, exact mass fragment information of these markers were obtained by UHPLC-Q-TOF-MS
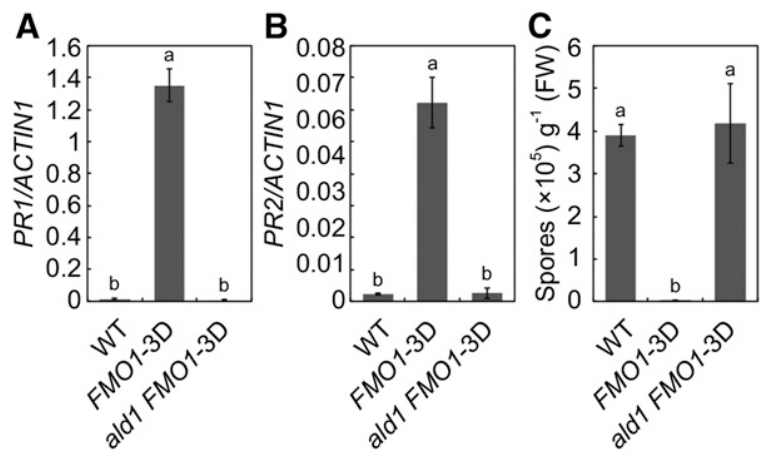

Figure 10. ALD1 Is Required for Constitutive Defense Responses in FM01-3D.

(A) and (B) $P R 1$ (A) and $P R 2$ (B) expression in 2-week-old seedlings of the wild type, FMO1-3D, and ald1 FMO1-3D determined by RT-qPCR. Values were obtained from abundances of $P R 1$ and $P R 2$ transcripts normalized against that of ACTIN1, respectively. Statistical differences among the samples are labeled with different letters $(P<0.01$, one-way ANOVA; $n=3)$. Similar results were obtained in three independent experiments.

(C) Growth of H.a. Noco2 on the wild type, FMO1-3D, and ald1 FMO1-3D. Three-week-old seedlings were sprayed with H.a. Noco2 spores $\left(5 \times 10^{4}\right.$ spores $/ \mathrm{mL}$ ). Infection was scored $7 \mathrm{~d}$ after inoculation by counting the numbers of spores per gram of leaf samples. Statistical differences between the samples are labeled with different letters $(P<0.01$, one-way ANOVA; $n=4$ ). Similar results were obtained in three independent experiments. 
analyses. For the separation, an Agilent 1290 Infinity UHPLC system was used with an Acquity UPLC HSS T3 column $(2.1 \times 100 \mathrm{~mm}, 1.8-\mu \mathrm{m}$ particle size; Waters) at $40^{\circ} \mathrm{C}$ and a flow rate of $0.5 \mathrm{~mL} / \mathrm{min}$. The solvent system and gradients were used as described for metabolite fingerprinting. Mass detection was performed with Agilent $6540 \mathrm{UH}$ AccurateMass-Q-TOF-MS. The MS was operated in positive and negative mode with Agilent Dual Jet Stream Technology (Agilent Technologies) as ESI source. Following ionization parameters were set: gas temperature, $300^{\circ} \mathrm{C}$; gas flow, $8 \mathrm{~L} / \mathrm{min}$; nebulizer pressure, 35 p.s.i.; sheath gas temperature, $350^{\circ} \mathrm{C}$; sheath gas flow, $11 \mathrm{~L} / \mathrm{min}$; Vcap, $3.5 \mathrm{kV}$; nozzle voltage, $100 \mathrm{~V}$. For isolation of precursor ions in the quadrupole, a mass window of 1.3 D was used. For data acquisition, Mass Hunter Workstation Acquisition software B.05.01 was used. Mass Hunter Qualitative Analysis software B.05.01 was used as analysis tool. Fragmentation of Pip ( $m / z$ 130.086), P2C ( $m / z 128.07)$, and ${ }^{15} \mathrm{~N},{ }^{13} \mathrm{C}$-labeled P2C ( $\left.\mathrm{m} / \mathrm{z} 130.070\right)$ and Pip $(m / z 132.086)$ were analyzed in positive ionization mode with a collision energy of $10 \mathrm{eV}$. Pip and L-lysine- $6-{ }^{13} \mathrm{C},{ }^{15} \mathrm{~N}$ hydrochloride were ordered from Sigma-Aldrich.

\section{Cloning and Expression of Arabidopsis ALD1 and SARD4}

For cloning of $A L D 1$ and SARD4, the coding sequences for both genes were amplified from Arabidopsis total CDNA using the primers ALD1-F1 and ALD1-R1 (for full-length ALD1) and SARD4-F and SARD4-R (for $S A R D 4)$. ALD1 was inserted into the pCDFDuet-1 vector (Novagen), whereas SARD4 was inserted into the pET24a vector (Novagen) utilizing EcoRI/Xhol restriction sites in both cases.

Proteins were expressed individually or jointly in Escherichia coli BL21* (DE3) cells. The bacterial cultures were incubated at $37^{\circ} \mathrm{C}$ until an $\mathrm{OD}_{600}$ of 0.6 to $0.8 \mathrm{AU}$ was reached. IPTG $(0.1 \mathrm{mM})$ was added for protein expression and the cultures continued to grow for additional $18 \mathrm{~h}$ at $16^{\circ} \mathrm{C}$. The expression of the heterologous proteins was verified by SDS-PAGE and immunoblot analysis. Samples $\left(\mathrm{V}=0.5 \mathrm{~mL} / \mathrm{OD}_{600}\right)$ were harvested by centrifugation ( $4 \mathrm{~min}$, $8,000 \mathrm{~g}$ ). The pellets were dissolved in $50 \mu \mathrm{L}$ water and mixed with $50 \mu \mathrm{L} 2 \times$ Laemmli buffer. Ten microliters of this solution were loaded on the SDSPAGE. The proteins were either visualized by Coomassie Brilliant Blue staining or blotted onto a nitrocellulose membrane. Tetra-His antibody (Qiagen; $0.1 \mu \mathrm{g} / \mathrm{mL}$ ) was used to detect the His-tagged proteins. A secondary anti-mouse antibody (Sigma-Aldrich) was used to visualize the proteins.

\section{In-Cell Activity Assay}

For the analysis of substrates and/or products of ALD1 (full length) and/or SARD4 catalyzed reactions, $900 \mu \mathrm{L}$ of the particular $E$. coli culture was mixed with $150 \mu \mathrm{L}$ methanol and $500 \mu \mathrm{L}$ methyl tert-butyl ether. After $45 \mathrm{~min}$ of shaking in the darkness at $4^{\circ} \mathrm{C}, 120 \mu \mathrm{L}$ water was added. The mixtures were centrifuged for $10 \mathrm{~min}$ at $16,000 \mathrm{~g}$ at $4^{\circ} \mathrm{C}$ for phase separation. Both phases were combined in one tube, whereby the interphase was discarded. The solvents were evaporated under a stream of nitrogen and the pellet subsequently resuspended in $30 \mu \mathrm{L}$ methanol/acetonitrile $(1: 1, v / v)$. After vigorous shaking, $100 \mu \mathrm{L}$ water was added. Insoluble residues were removed by $10 \mathrm{~min}$ centrifugation at $16,000 \mathrm{~g}$. The supernatant was transferred into glass vials for UPLC-Q-TOF-MS analyses.

\section{Purification and Activity Assay of Arabidopsis ALD1}

For the protein purification, we followed the protocol of Sobolev et al. (2013). A truncated version of ALD1 (420-ALD1, amino acids 21 to 456) was cloned into pET28 vector (Novagen) from the earlier mentioned pCDFALD1 plasmid using the primers ALD1-F2 and ALD1-R2. Expression was performed as described before. The cells were disrupted with pulsed ultrasonic waves (Branson Sonifier Cell Disruptor B15; Branson Ultrasonics) in a solution containing $50 \mathrm{mM}$ Tris, $\mathrm{pH} 7.2,500 \mathrm{mM} \mathrm{NaCl}, 1 \mathrm{mM} \mathrm{PMSF}$, and $1 \mathrm{mM}$ DTT. Cell debris was removed by centrifugation $\left(50,000 \mathrm{~g}, 4^{\circ} \mathrm{C}\right.$,
$30 \mathrm{~min}$ ). The supernatant was subsequently applied onto a HisTrap column (GE Healthcare) equilibrated with $50 \mathrm{mM}$ Tris, pH 7.5, $200 \mathrm{mM} \mathrm{NaCl}, 5 \mathrm{mM}$ imidazole, and $1 \mathrm{mM}$ DTT. For the elution, $30 \%$ elution buffer $(50 \mathrm{mM}$ Tris, $\mathrm{pH} 7.5,200 \mathrm{mM} \mathrm{NaCl}, 500 \mathrm{mM}$ imidazole, and $1 \mathrm{mM}$ DTT) was used.

For the activity assay, $20 \mu \mathrm{g} \Delta 20-\mathrm{ALD} 1$ was added to $250 \mu \mathrm{L}$ of reaction buffer which contained $50 \mathrm{mM}$ Tris, $\mathrm{pH} 7.2,100 \mathrm{mM} \mathrm{NaCl}, 50 \mathrm{mM}$ lysine, $50 \mu \mathrm{M}$ lysine $-6-{ }^{13} \mathrm{C},-{ }^{15} \mathrm{~N}, 50 \mathrm{mM}$ pyruvate, and $2 \mu \mathrm{M}$ pyridoxal phosphate. The reaction was performed for $3 \mathrm{~h}$ at $30^{\circ} \mathrm{C}$, shaking at $150 \mathrm{rpm}$. To remove the protein from the solution, we used filter centrifugation $\left(4^{\circ} \mathrm{C}, 4000 \mathrm{~g}\right)$ with a SpinX UF concentrator (10,000 MWCO; Corning). The product was then analyzed with either high-resolution MS/MS or used for the colorimetric analysis. Treatment with o-aminobenzaldehyde (o-AB; Sigma-Aldrich) was used to distinguish between P6C and P2C (Soda et al., 1968). One hundred microliters of the sample was incubated with $890 \mu \mathrm{L}$ sodium acetate buffer $(0.2 \mathrm{M}, \mathrm{pH} 5)$ and $10 \mu \mathrm{Lo}-\mathrm{AB}(0.4 \mathrm{M})$ for $1 \mathrm{~h}$ at $37^{\circ} \mathrm{C}$. The absorbance was measured with a Carry 100 UV-Vis spectrophotometer (Agilent) in a range of 350 to $600 \mathrm{~nm}$. Reaction buffer alone was used as the control.

\section{Purification and Activity Assay of Arabidopsis SARD4}

SARD4 was expressed as described above. For the protein purification, the same protocol as for the $\triangle 20-A L D 1$ purification was used. For the activity assay of SARD4, the substrate P2C was generated by the $\Delta 20$ ALD1 reaction using either lysine or lysine $-6-{ }^{13} \mathrm{C},-{ }^{-15} \mathrm{~N}$ as substrate. $\triangle 20-A L D 1$ was removed from the reaction solution by filter centrifugation as described above. The presence of $\mathrm{P} 2 \mathrm{C}$ in the solution was confirmed by UPLC-Q-TOF-MS analyses. Purified SARD4 was added to $250 \mu \mathrm{L}$ of the reaction buffer containing $50 \mathrm{mM}$ Tris, $\mathrm{pH} 7.2,100 \mathrm{mM}$ $\mathrm{NaCl}$, and $2 \mu \mathrm{M} \mathrm{NADPH}$. The reaction was started by adding $100 \mu \mathrm{L}$ of the protein-free P2C solution from the activity assay of $\triangle 20$-ALD1. The reaction was performed for $3 \mathrm{~h}$ at $30^{\circ} \mathrm{C}$, shaking at $150 \mathrm{rpm}$. Subsequently, the reaction solution was centrifuged for $10 \mathrm{~min}(16,000 \mathrm{~g}$, $4^{\circ} \mathrm{C}$ ) and the product solution was examined by UPLC-Q-TOF-MS analyses. The identity of Pip as the product of the SARD4-catalyzed reaction was confirmed by MS/MS fragmentation analyses and comparison of its retention time with the commercial Pip. Pip standard solution $(1 \mu \mathrm{M})$ was used in the analysis.

\section{Accession Numbers}

Sequence data from this article can be found in the Arabidopsis Genome Initiative or GenBank/EMBL databases under the following accession numbers: AT1G19250 (FMO1), AT5G52810 (SARD4), AT2G13810 (ALD1), AAF08790 (RPP5), AT1G64280 (NPR1), At2g14610 (PR1), At3g57260 (PR2), and At2g37620 (ACTIN1).

\section{Supplemental Data}

Supplemental Figure 1. SARD4 is not required for local SA accumulation induced by P.s.m. ES4326.

Supplemental Figure 2. SARD4 is not required for local $P R$ gene expression induced by P.s.m. ES4326.

Supplemental Figure 3. SARD4 is not required for local resistance against P.s.m. ES4326.

Supplemental Figure 4. Heterologous expression of PCDF-ALD1 and pET-SARD4 in a single $E$. coli liquid culture for the in-cell activity assay.

Supplemental Figure 5. MS/MS fragmentation patterns of Pip and P2C from in-cell assay.

Supplemental Figure 6. SDS-PAGE analysis of heterologous expressed $6 \times$ His-tagged $\triangle 20$ ALD1 protein $(\sim 50 \mathrm{kD})$ purified by affinity chromatography. 
Supplemental Figure 7. SDS-PAGE analysis of heterologous expressed $6 \times$ His-tagged SARD4 protein $(\sim 36.4 \mathrm{kD})$ purified by affinity chromatography.

Supplemental Table 1. Primers used in this study.

Supplemental Table 2. ANOVA tables for statistical analysis.

\section{ACKNOWLEDGMENTS}

I.F. was supported by the Deutsche Forschungsgemeinschaft (ZUK 45/ 2010 and partially by IRTG 2172 "PRoTECT"). S.H. and D.R. were supported by the "PRoTECT" program of the Göttingen Graduate School of Neuroscience and Molecular Biology. Y.Z. was supported by Natural Sciences and Engineering Research Council of Canada, Canada Foundation for Innovation, and British Columbia Knowledge Development Fund.

\section{AUTHOR CONTRIBUTIONS}

P.D., D.R., Y.D., K.F., X.L., R.J., I.F., and Y.Z. conceived and designed the experiments. P.D., D.R., Y.D., K.F., L.B., S.H., and S.X. performed the experiments. P.D., D.R., Y.D., K.F., I.F., and Y.Z. analyzed the data, P.D., D.R., Y.D., K.F., L.B., I.F., and Y.Z. wrote the article.

Received June 15, 2016; revised September 21, 2016; accepted October 5, 2016; published October 6, 2016.

\section{REFERENCES}

Bartsch, M., Gobbato, E., Bednarek, P., Debey, S., Schultze, J.L., Bautor, J., and Parker, J.E. (2006). Salicylic acid-independent ENHANCED DISEASE SUSCEPTIBILITY1 signaling in Arabidopsis immunity and cell death is regulated by the monooxygenase FMO1 and the Nudix hydrolase NUDT7. Plant Cell 18: 1038-1051.

Bernsdorff, F., Döring, A.-C., Gruner, K., Schuck, S., Bräutigam, A., and Zeier, J., (2016). Pipecolic acid orchestrates plant systemic acquired resistance and defense priming via salicylic acid-dependent and independent pathways. Plant Cell 28: 102-129.

Bi, D., Cheng, Y.T., Li, X., and Zhang, Y. (2010). Activation of plant immune responses by a gain-of-function mutation in an atypical receptor-like kinase. Plant Physiol. 153: 1771-1779.

Bruckhoff, V., Haroth, S., Feussner, K., König, S., Brodhun, F., and Feussner, I. (2016). Functional characterization of CYP94 genes and identification of a novel jasmonate catabolite in flowers. PLoS One 11: e0159875.

Cecchini, N.M., Jung, H.W., Engle, N.L., Tschaplinski, T.J., and Greenberg, J.T. (2015). ALD1 regulates basal immune components and early inducible defense responses in Arabidopsis. Mol. Plant Microbe Interact. 28: 455-466.

Champigny, M.J., Isaacs, M., Carella, P., Faubert, J., Fobert, P.R., and Cameron, R.K. (2013). Long distance movement of DIR1 and investigation of the role of DIR1-like during systemic acquired resistance in Arabidopsis. Front. Plant Sci. 4: 230.

Chanda, B., Xia, Y., Mandal, M.K., Yu, K., Sekine, K.T., Gao, Q.M., Selote, D., Hu, Y., Stromberg, A., Navarre, D., Kachroo, A., and Kachroo, P. (2011). Glycerol-3-phosphate is a critical mobile inducer of systemic immunity in plants. Nat. Genet. 43: 421-427.

Chaturvedi, R., Venables, B., Petros, R.A., Nalam, V., Li, M., Wang, X., Takemoto, L.J., and Shah, J. (2012). An abietane diterpenoid is a potent activator of systemic acquired resistance. Plant J. 71: 161-172.
Fu, Z.Q., and Dong, X. (2013). Systemic acquired resistance: turning local infection into global defense. Annu. Rev. Plant Biol. 64: 839-863.

Garcion, C., Lohmann, A., Lamodière, E., Catinot, J., Buchala, A., Doermann, P., and Métraux, J.P. (2008). Characterization and biological function of the ISOCHORISMATE SYNTHASE2 gene of Arabidopsis. Plant Physiol. 147: 1279-1287.

Jing, B., Xu, S., Xu, M., Li, Y., Li, S., Ding, J., and Zhang, Y. (2011). Brush and spray: a high-throughput systemic acquired resistance assay suitable for large-scale genetic screening. Plant Physiol. 157: 973-980.

Jung, H.W., Tschaplinski, T.J., Wang, L., Glazebrook, J., and Greenberg, J.T. (2009). Priming in systemic plant immunity. Science 324: 89-91.

Kaever, A., Landesfeind, M., Feussner, K., Mosblech, A., Heilmann, I., Morgenstern, B., Feussner, I., and Meinicke, P. (2015). MarVis-Pathway: integrative and exploratory pathway analysis of non-targeted metabolomics data. Metabolomics 11: 764-777.

Koch, M., Vorwerk, S., Masur, C., Sharifi-Sirchi, G., Olivieri, N., and Schlaich, N.L. (2006). A role for a flavin-containing mono-oxygenase in resistance against microbial pathogens in Arabidopsis. Plant J. 47: 629-639.

König, S., Feussner, K., Schwarz, M., Kaever, A., Iven, T., Landesfeind, M., Ternes, P., Karlovsky, P., Lipka, V., and Feussner, I. (2012). Arabidopsis mutants of sphingolipid fatty acid $\alpha$-hydroxylases accumulate ceramides and salicylates. New Phytol. 196: 1086-1097.

Maldonado, A.M., Doerner, P., Dixon, R.A., Lamb, C.J., and Cameron, R.K. (2002). A putative lipid transfer protein involved in systemic resistance signalling in Arabidopsis. Nature 419: 399-403.

Mishina, T.E., and Zeier, J. (2006). The Arabidopsis flavin-dependent monooxygenase FMO1 is an essential component of biologically induced systemic acquired resistance. Plant Physiol. 141: 16661675.

Návarová, H., Bernsdorff, F., Döring, A.C., and Zeier, J. (2012). Pipecolic acid, an endogenous mediator of defense amplification and priming, is a critical regulator of inducible plant immunity. Plant Cell 24: 5123-5141.

Park, S.-W., Kaimoyo, E., Kumar, D., Mosher, S., and Klessig, D.F. (2007). Methyl salicylate is a critical mobile signal for plant systemic acquired resistance. Science 318: 113-116.

Parker, J.E., Coleman, M.J., Szabò, V., Frost, L.N., Schmidt, R., van der Biezen, E.A., Moores, T., Dean, C., Daniels, M.J., and Jones, J.D. (1997). The Arabidopsis downy mildew resistance gene RPP5 shares similarity to the toll and interleukin-1 receptors with $N$ and L6. Plant Cell 9: 879-894.

Sharma, S., Shinde, S., and Verslues, P.E. (2013). Functional characterization of an ornithine cyclodeaminase-like protein of Arabidopsis thaliana. BMC Plant Biol. 13: 182.

Sobolev, V., Edelman, M., Dym, O., Unger, T., Albeck, S., Kirma, M., and Galili, G. (2013). Structure of ALD1, a plant-specific homologue of the universal diaminopimelate aminotransferase enzyme of lysine biosynthesis. Acta Crystallogr. Sect. F Struct. Biol. Cryst. Commun. 69: 84-89.

Soda, K., Misono, H., and Yamamoto, T. (1968). L-Lysine:alphaketoglutarate aminotransferase. I. Identification of a product, delta1-piperideine-6-carboxylic acid. Biochemistry 7: 4102-4109.

Song, J.T., Lu, H., and Greenberg, J.T. (2004a). Divergent roles in Arabidopsis thaliana development and defense of two homologous genes, aberrant growth and death2 and AGD2-LIKE DEFENSE RESPONSE PROTEIN1, encoding novel aminotransferases. Plant Cell 16: 353-366. 
Song, J.T., Lu, H., McDowell, J.M., and Greenberg, J.T. (2004b). A key role for ALD1 in activation of local and systemic defenses in Arabidopsis. Plant J. 40: 200-212.

Sun, T., Zhang, Y., Li, Y., Zhang, Q., Ding, Y., and Zhang, Y. (2015). ChIP-seq reveals broad roles of SARD1 and CBP60g in regulating plant immunity. Nat. Commun. 6: 10159.

Vlot, A.C., Dempsey, D.A., and Klessig, D.F. (2009). Salicylic acid, a multifaceted hormone to combat disease. Annu. Rev. Phytopathol. 47: 177-206.

Yu, K., Soares, J.M., Mandal, M.K., Wang, C., Chanda, B., Gifford, A.N., Fowler, J.S., Navarre, D., Kachroo, A., and Kachroo, P. (2013). A feedback regulatory loop between G3P and lipid transfer proteins DIR1 and AZI1 mediates azelaic-acid-induced systemic immunity. Cell Reports 3: 1266-1278.
Zeier, J. (2013). New insights into the regulation of plant immunity by amino acid metabolic pathways. Plant Cell Environ. 36: 2085-2103.

Zhang, Y., Glazebrook, J., and Li, X. (2007). Identification of components in disease-resistance signaling in Arabidopsis by mapbased cloning. Methods Mol. Biol. 354: 69-78.

Zhang, Y., Tessaro, M.J., Lassner, M., and Li, X. (2003). Knockout analysis of Arabidopsis transcription factors TGA2, TGA5, and TGA6 reveals their redundant and essential roles in systemic acquired resistance. Plant Cell 15: 2647-2653.

Zhang, Y., Xu, S., Ding, P., Wang, D., Cheng, Y.T., He, J., Gao, M., Xu, F., Li, Y., Zhu, Z., Li, X., and Zhang, Y. (2010). Control of salicylic acid synthesis and systemic acquired resistance by two members of a plant-specific family of transcription factors. Proc. Natl. Acad. Sci. USA 107: 18220-18225. 

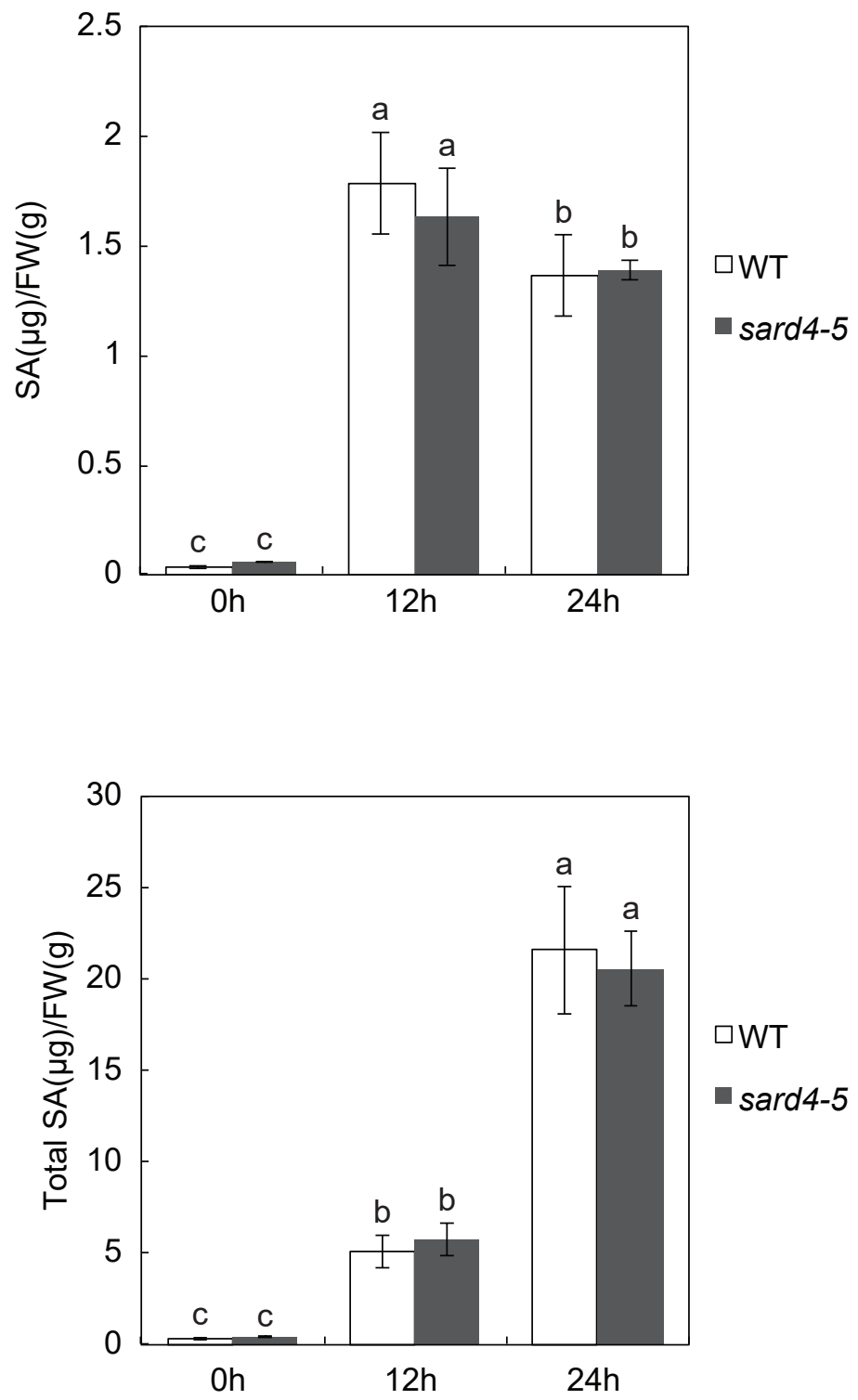

Supplemental Figure 1. SARD4 is not required for local SA accumulation induced by P.s.m. ES4326. Leaves of four-week-old plants were infiltrated with P.s.m. ES4326 $\left(\mathrm{OD}_{600}=0.005\right)$, and the inoculated leaves were collected $12 \mathrm{~h}$ and $24 \mathrm{~h}$ llater for SA extraction. Free SA and total SA levels were quantified by high-performance liquid chromatography. Statistical differences between the samples are labeled with different letters $(P<0.05$, one-way ANOVA; $n=4)$. The experiment was repeated twice with similar results. 


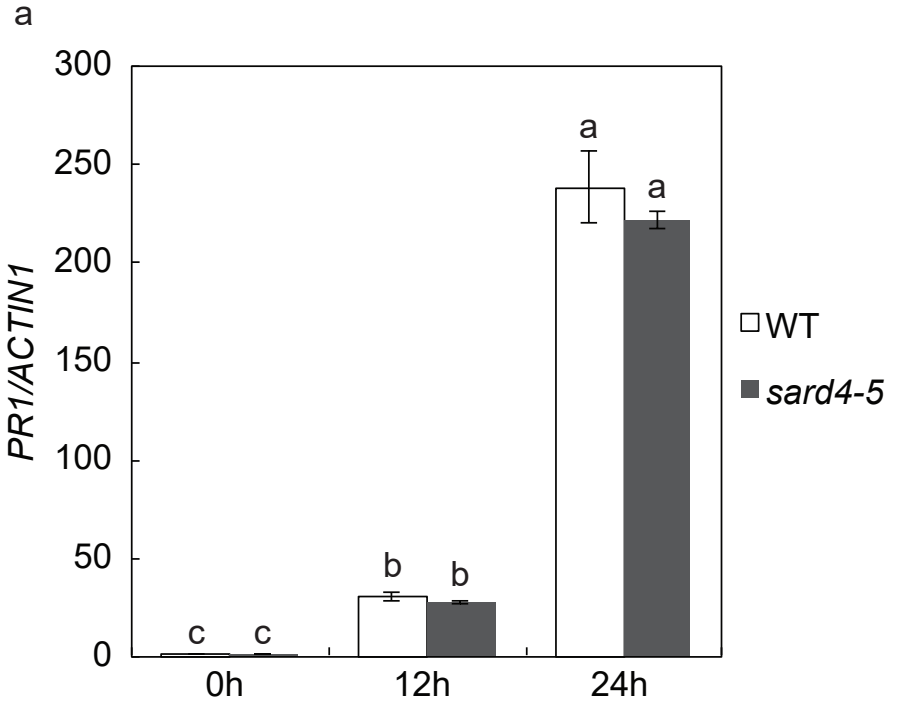

b

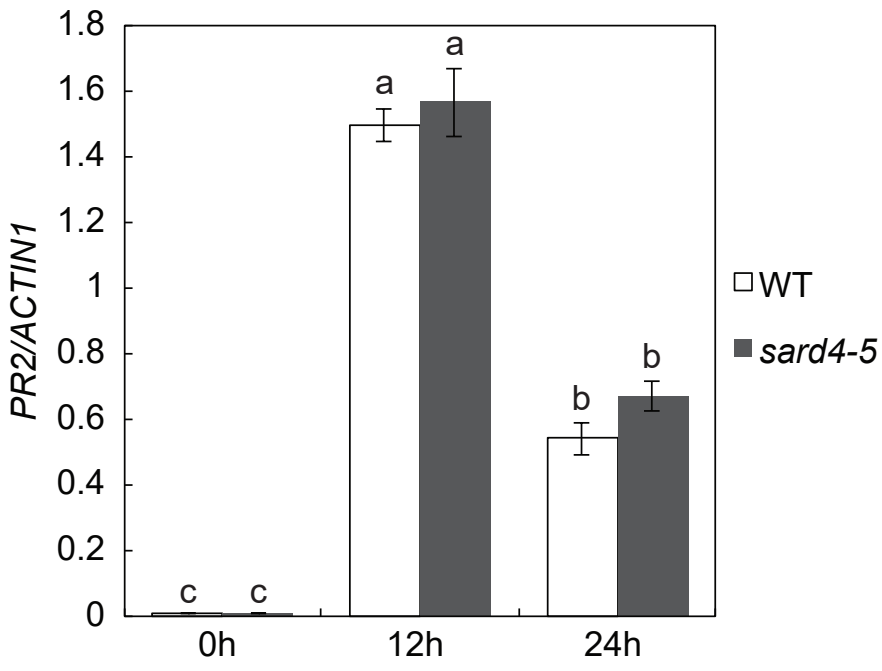

Supplemental Figure 2. SARD4 is not required for local $P R$ gene expression induced by P.s.m. ES4326. $P R 1$ (a) and PR2 (b) expression in WT and sard4-5 induced by P.s.m. ES4326. Three-week-old plants were infiltrated with P.s.m. ES4326 $\left(\mathrm{OD}_{600}=0.005\right)$, and the inoculated leaves were collected $12 \mathrm{~h}$ and $24 \mathrm{~h}$ later for RT-qPCR analysis. Values were obtained from abundances of $P R 1$ and $P R 2$ transcripts normalized against that of ACTIN1.

Statistical differences among the samples are labeled with different letters $(P<0.01$, one-way ANOVA; $n=3)$. Similar results were obtained in three independent experiments. 


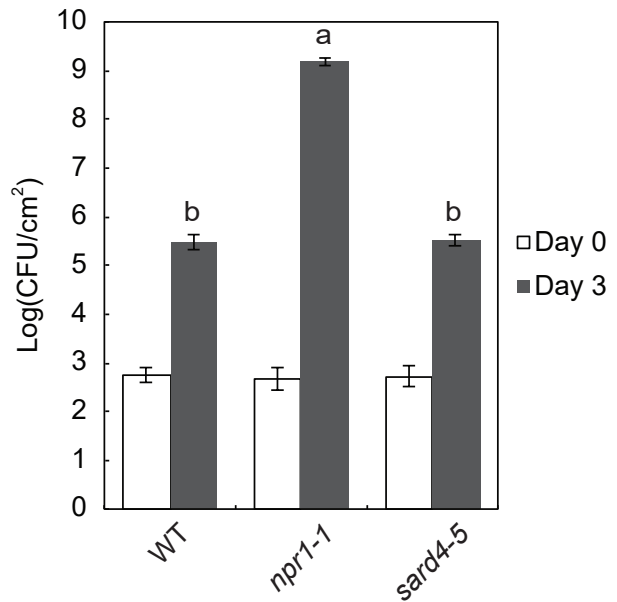

Supplemental Figure 3. SARD4 is not required for local resistance against P.s.m. ES4326.

Leaves of four-week-old WT, npr1-1 and sard4-5 plants were infiltrated with a P.s.m. ES4326 (OD $600=0.0001)$ Bacterial growth was determined three days post inoculation. cfu, colony forming unit. Statistical differences between the samples are labeled with different letters $(P<0.01$, one-way ANOVA; $n=6)$. The experiment was repeated twice with similar results. 


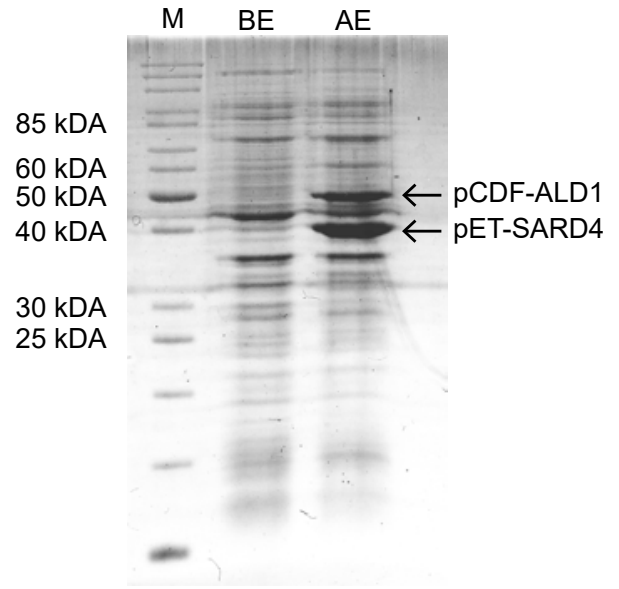

Supplemental Figure 4. Heterologous expression of pCDF-ALD1 and pET-SARD4 in a single E.coli liquid culture for the in-cell activity assay.

Coomassie brilliant blue-stained SDS-PAGE showing proteins before $(B E)$ and after induction $(\mathrm{AE})$ of protein expression. Proteins of the correct molecular weight (ALD1 in pCDFDuet vector, $51.6 \mathrm{kDa}$ respectively SARD4 in pET24, $38.0 \mathrm{kDa}$ ) are marked with black arrows.

Similar results were obtained in two independent experiments. 

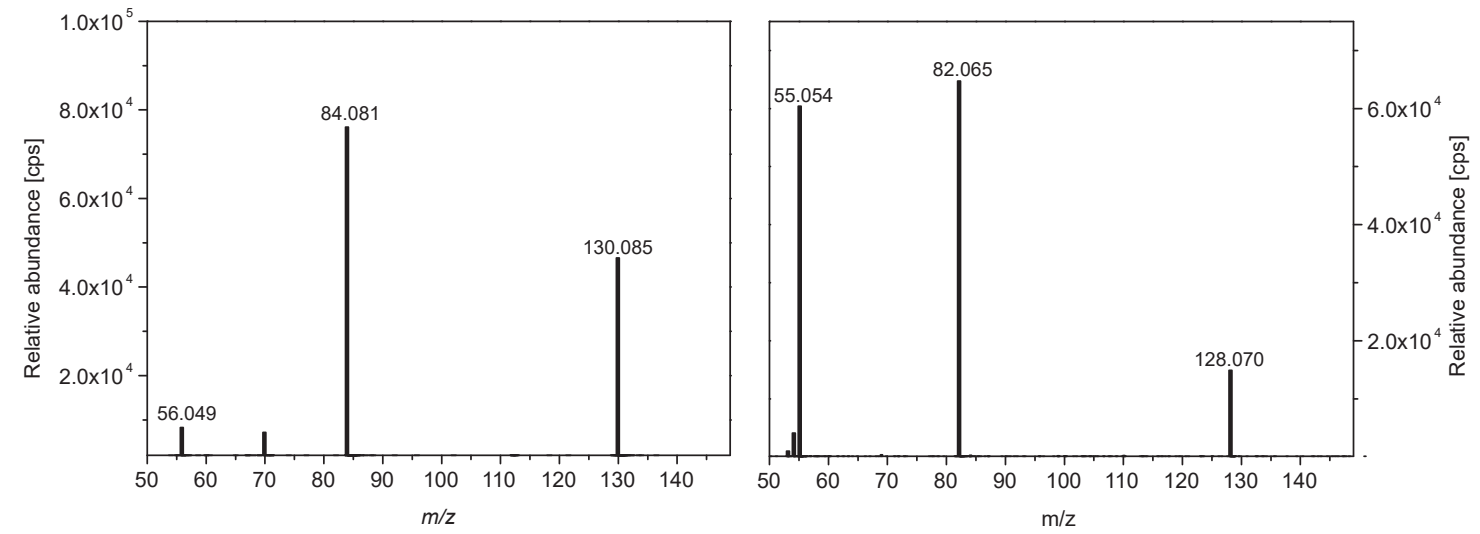

Supplemental Figure 5. MS/MS fragmentation patterns of Pip and P2C from in-cell assay.

MS/MS fragmentation pattern of (a) Pip and (b) P2C detected in the E. coli cultures expressing only ALD1 (a), and

ALD1 and SARD4 together (b), respectively. The fragmentation of Pip (a) and P2C (b) leads to a loss of the carboxyl group $\left(\mathrm{m} / \mathrm{z} 84.081\right.$ and $\mathrm{m} / \mathrm{z} 82.065$ respectively). In Pip, the mass signal of $\mathrm{m} / \mathrm{z} 56.049$ represents a $\mathrm{C}_{3} \mathrm{H}_{6} \mathrm{~N}$-fragment. In P2C the $\mathrm{C}=\mathrm{N}$ double bond stays intact so that the mass signal of $\mathrm{m} / \mathrm{z} 55.054$ represents a $\mathrm{C}_{4} \mathrm{H}_{7}$-fragment. 


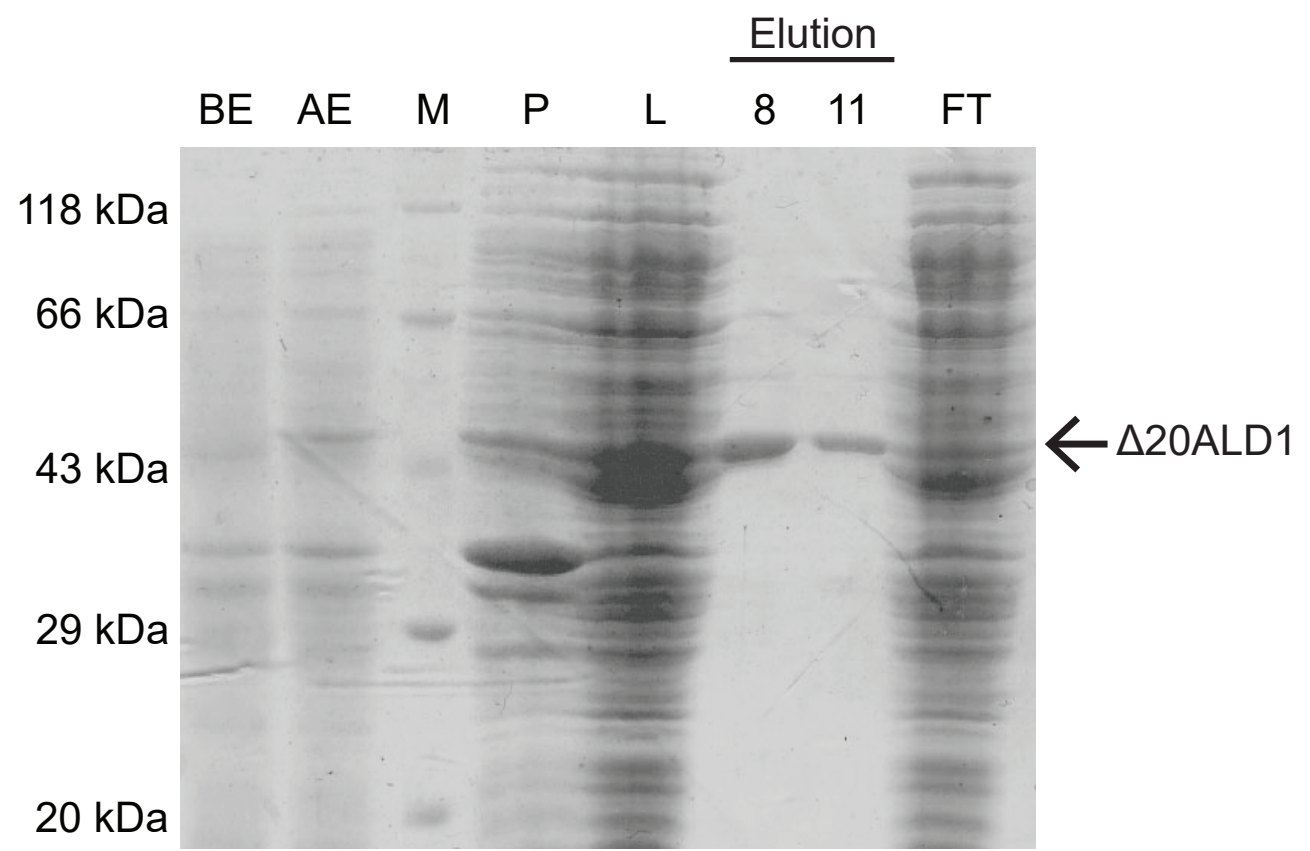

Supplemental Figure 6. SDS-PAGE analysis of heterologous expressed 6×His-tagged $\triangle 20 A L D 1$ protein $(\sim 50 \mathrm{kDa})$ purified by affinity chromatography.

Coomassie brilliant blue-stained SDS-PAGE gel shows total proteins before (BE) and after induction (AE) of $\triangle 20 \mathrm{ALD} 1$ expression in E.coli and different fractions from the purification process. Protein bands of the correct molecular weight are marked with a black arrow. M, Molecular marker; P, pellet fraction; L, lysate fraction before purification; FT, collected flow through. The results are representative for three independent experiments. 


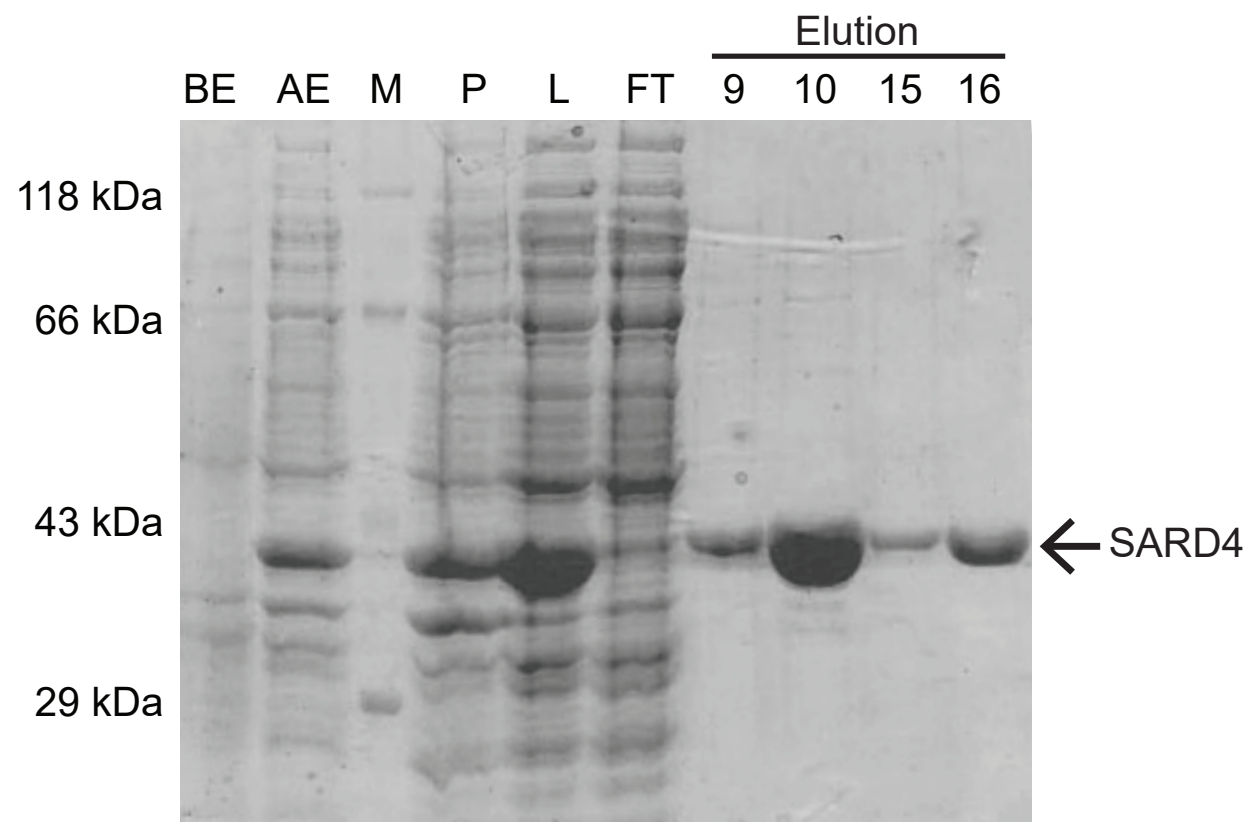

Supplemental Figure 7. SDS-PAGE analysis of heterologous expressed 6xHis-tagged SARD4 protein ( 36.4 kDa) purified by affinity chromatography.

Coomassie brilliant blue-stained SDS-PAGE gel shows total proteins before (BE) and after induction (AE) of SARD4 expression in E.coli and different fractions from the purification process. Protein bands of the correct molecular weight are marked with a black arrow. M, Molecular marker; P, pellet fraction; L, lysate fraction before purification; FT, collected flow through. The results are representative for three independent experiments. 
Supplemental Table 1. Primers used in this study.

\begin{tabular}{|l|l|l|}
\hline Primer & 5'-3' sequence & Purpose \\
\hline GABI_428E01-F & tacaccgtttcatcaccttc & Genotyping \\
\hline GABI_428E01-R & cccacaaatgtcttctctc & Genotyping \\
\hline SALK_007673-F & ggttattggtacttacttggag & Genotyping \\
\hline SALK_007673-R & agaagaaatactctatccggg & Genotyping \\
\hline RT-SARD4-F & aaggagatttcattcgatagcc & RT \\
\hline RT-SARD4-R & ctgaggtaagtctcgtgaac & RT \\
\hline K19E20-F & gacaagaaccacatgagagc & Mapping \\
\hline K19E20-R & gttatgtgtacacttcaggtc & Mapping \\
\hline MMN10-F & agctgcaataatgccaaagg & Mapping \\
\hline MMN10-R & gaaccatcaccactggtgag & Mapping \\
\hline MYN8-F & agtcgtcatgctcatgaacc & Mapping \\
\hline MYN8-R & ttcacaagggattaagaacc & Mapping \\
\hline K10D11-F & ctgattcagcacaggtaaca & Mapping \\
\hline K10D11-R & tctacccaaacggctcacca & Mapping \\
\hline ALD1-F1 & acggaattcatggtcagtctaatgttctttagttc & cloning \\
\hline ALD1-R1 & acgctcgagattggtattagaagtggaagagag & cloning \\
\hline SARD4-F & cggaattcatggctgcattaccagtatt & cloning \\
\hline SARD4-R & acgctcgagacaacggctgaggtaagtct & cloning \\
\hline ALD1-F2 & acgggatccatgcccaaggctagtttggacttc & cloning \\
\hline ALD1-R2 & acgaagcttctaattggtattagaagtggaag & cloning \\
\hline
\end{tabular}


Supplemental ANOVA tables

ANOVA for Figure 1B

\begin{tabular}{cccccc}
\hline & df & SS & MS & F-value & P-value \\
\hline genotype & 3 & 157.7734 & 52.5911 & 34.903 & $3.31 \mathrm{E}-06$ \\
residuals & 12 & 18.0813 & 1.5068 & & \\
\hline
\end{tabular}

\section{ANOVA for Figure 1C}

\begin{tabular}{lccccc}
\hline & df & SS & MS & F-value & P-value \\
\hline genotype & 3 & 7.2596 & 2.4199 & 130.1571 & $3.97 \mathrm{E}-07$ \\
residuals & 8 & 0.1487 & 0.0186 & & \\
\hline
\end{tabular}

\section{ANOVA for Figure 1D}

\begin{tabular}{cccccc}
\hline & df & SS & MS & F-value & P-value \\
\hline genotype & 3 & 0.0225 & 0.0075 & 94.7537 & $1.37 \mathrm{E}-06$ \\
residuals & 8 & 0.0006 & 0.0001 & & \\
\hline
\end{tabular}

ANOVA for Figure 2B

\begin{tabular}{cccccc}
\hline & df & SS & MS & F-value & P-value \\
\hline time & 1 & 0 & 0 & $2,550.11$ & $9.20 \mathrm{E}-07$ \\
residuals & 4 & 0 & 0 & & \\
\hline
\end{tabular}

ANOVA for Figure 2C

\begin{tabular}{cccccc}
\hline & df & SS & MS & F-value & P-value \\
\hline genotype & 2 & 574.9904 & 287.4952 & 33.7775 & $6.55 \mathrm{E}-05$ \\
residuals & 9 & 76.6029 & 8.5114 & & \\
\hline
\end{tabular}

Tukey's unequal N HSD groups for Figure 1B

\begin{tabular}{lcc}
\hline genotype & mean & group \\
\hline WT & 9.0202 & $\mathrm{a}$ \\
sard4-4 FMO1-3D & 4.5914 & $\mathrm{~b}$ \\
sard4-3 FMO1-3D & 3.8046 & $\mathrm{~b}$ \\
FMO1-3D & 0.1921 & $\mathrm{c}$ \\
\hline
\end{tabular}

Tukey's unequal N HSD groups for Figure 1C

\begin{tabular}{lcc}
\hline genotype & mean & group \\
\hline FMO1-3D & 1.822 & $\mathrm{a}$ \\
sard4-3 FMO1-3D & 0.048 & $\mathrm{~b}$ \\
sard4-4 FMO1-3D & 0.029 & $\mathrm{~b}$ \\
WT & 0.002 & $\mathrm{~b}$ \\
\hline
\end{tabular}

Tukey's unequal N HSD groups for Figure 1D

\begin{tabular}{lcc}
\hline genotype & mean & group \\
\hline FMO1-3D & 0.104 & $\mathrm{a}$ \\
sard4-3 FMO1-3D & 0.011 & $\mathrm{~b}$ \\
sard4-4 FMO1-3D & 0.002 & $\mathrm{~b}$ \\
WT & 0.001 & $\mathrm{~b}$ \\
\hline
\end{tabular}

Tukey's unequal N HSD groups for Figure 2B

\begin{tabular}{lcc}
\hline time & mean & group \\
\hline $24 \mathrm{~h}$ & 0.0019 & $\mathrm{a}$ \\
Oh & 0 & $\mathrm{~b}$ \\
\hline
\end{tabular}

Tukey's unequal N HSD groups for Figure2C

\begin{tabular}{lcc}
\hline treatment & mean & group \\
\hline WT & 16.5376 & $\mathrm{a}$ \\
sard4-5 FMO1-3D & 13.631 & $\mathrm{a}$ \\
FMO1-3D & 0.6176 & $\mathrm{~b}$ \\
\hline
\end{tabular}


ANOVA for Figure 3A, Free SA

\begin{tabular}{lccccc}
\hline & df & SS & MS & F-value & P-value \\
\hline genotype & 1 & 0.0008 & 0.0008 & 6.3293 & 0.0247 \\
treatment & 1 & 0.0011 & 0.0011 & 10.9858 & 0.0051 \\
genotype $\times$ treatment & 3 & 0.002 & 0.0007 & 23.3953 & $2.66 \mathrm{E}-05$ \\
residuals & 12 & 0 & 0 & & \\
\hline
\end{tabular}

Tukey's HSD groups for Figure 3A, Free SA

\begin{tabular}{lcc}
\hline genotype $\times$ treatment & mean & group \\
\hline WT P.s.m. & 0.062 & $\mathrm{a}$ \\
sard4-5 P.s.m. & 0.04 & $\mathrm{~b}$ \\
WT mock & 0.037 & $\mathrm{~b}$ \\
sard4-5 mock & 0.031 & $\mathrm{~b}$ \\
\hline
\end{tabular}

ANOVA for Figure 3A, Total SA

\begin{tabular}{lccccc}
\hline & df & SS & MS & F-value & P-value \\
\hline genotype & 1 & 0.6799 & 0.6799 & 8.735 & 0.0104 \\
treatment & 1 & 0.676 & 0.676 & 8.6532 & 0.0107 \\
genotype $\times$ treatment & 3 & 1.7485 & 0.5828 & 331.403 & $8.39 \mathrm{E}-12$ \\
residuals & 12 & 0.0211 & 0.0018 & & \\
\hline
\end{tabular}

\section{Tukey's HSD groups for Figure 3A,Total SA}

\begin{tabular}{lcc}
\hline genotype $\times$ treatment & mean & group \\
\hline WT P.s.m. & 1.6071 & $\mathrm{a}$ \\
WT mock & 0.8827 & $\mathrm{~b}$ \\
sard4-5 P.s.m. & 0.8815 & $\mathrm{~b}$ \\
sard4-5 mock & 0.7837 & $\mathrm{~b}$ \\
\hline
\end{tabular}

ANOVA for Figure 3B

\begin{tabular}{lccccc}
\hline & df & SS & MS & F-value & P-value \\
\hline genotype & 1 & 6.8665 & 6.8665 & 1.5402 & 0.2429 \\
treatment & 1 & 37.7423 & 37.7423 & 27.5373 & 0.0004 \\
genotype $\times$ treatment & 3 & 51.2747 & 17.0916 & 788.0954 & $3.18 \mathrm{E}-10$ \\
residuals & 8 & 0.1735 & 0.0217 & & \\
\hline
\end{tabular}

Tukey's HSD groups for Figure 3B

\begin{tabular}{lcc}
\hline genotype $\times$ treatment & mean & group \\
\hline WT P.s.m. & 5.132 & $\mathrm{a}$ \\
sard4-5 P.s.m. & 2.1285 & $\mathrm{~b}$ \\
WT mock & 0.0944 & $\mathrm{C}$ \\
sard4-5 mock & 0.0721 & $\mathrm{c}$ \\
\hline
\end{tabular}


ANOVA for Figure 3C

\begin{tabular}{lccccc}
\hline & df & SS & MS & F-value & P-value \\
\hline genotype & 1 & 1.0208 & 1.0208 & 1.5988 & 0.2347 \\
treatment & 1 & 5.4675 & 5.4675 & 28.2096 & 0.0003 \\
genotype $\times$ treatment & 3 & 7.3849 & 2.4616 & 946.7778 & $1.53 \mathrm{E}-10$ \\
residuals & 8 & 0.0208 & 0.0026 & & \\
\hline
\end{tabular}

ANOVA for Figure 3D

\begin{tabular}{lccccc}
\hline & df & SS & MS & F-value & P-value \\
\hline genotype & 2 & 2.8929 & 1.4464 & 13.9718 & $4.02 \mathrm{E}-05$ \\
treatment & 1 & 1.5422 & 1.5422 & 10.9998 & 0.0022 \\
genotype $\times$ treatment & 5 & 6.2011 & 1.2402 & 344.0076 & $1.11 \mathrm{E}-16$ \\
residuals & 30 & 0.1082 & 0.0036 & & \\
\hline
\end{tabular}

Tukey's HSD groups for Figure 3C

\begin{tabular}{lcc}
\hline genotype $\times$ treatment & mean & group \\
\hline WT P.s.m. & 1.9667 & $\mathrm{a}$ \\
sard4-5 P.s.m. & 0.8367 & $\mathrm{~b}$ \\
WT mock & 0.07 & $\mathrm{c}$ \\
sard4-5 mock & 0.0333 & $\mathrm{c}$ \\
\hline
\end{tabular}

Tukey's HSD groups for Figure 3D

\begin{tabular}{lcc}
\hline genotype $\times$ treatment & mean & group \\
\hline fmo1 P.s.m. & 6.290538667 & $\mathrm{a}$ \\
fmo1 mock & 6.278133167 & $\mathrm{a}$ \\
sard4-5 mock & 6.111863833 & $\mathrm{~b}$ \\
WT mock & 6.1055395 & $\mathrm{~b}$ \\
sard4-5 P.s.m. & 5.882202167 & $\mathrm{c}$ \\
WT P.s.m. & 5.0809245 & $\mathrm{~d}$ \\
\hline
\end{tabular}

\section{ANOVA for Figure 4A}

\begin{tabular}{lccccc}
\hline & $\mathrm{df}$ & $\mathrm{SS}$ & $\mathrm{MS}$ & F-value & P-value \\
\hline genotype & 2 & $775,790.78$ & $387,895.39$ & 14.5588 & $2.93 \mathrm{E}-05$ \\
treatment & 1 & $315,940.38$ & $315,940.38$ & 8.0219 & 0.0077 \\
genotype $\times$ treatment & 5 & $1,578,159.64$ & $315,631.93$ & 123.1973 & $1.11 \mathrm{E}-16$ \\
residuals & 30 & $76,860.13$ & $2,562.00$ & & \\
\hline
\end{tabular}

\section{Tukey's HSD groups for Figure 4A}

\begin{tabular}{lcc}
\hline genotype $\times$ treatment & mean & group \\
\hline WT P.s.m. & 620.6161583 & $\mathrm{a}$ \\
WT mock & 104.806915 & $\mathrm{~b}$ \\
sard4-5 P.s.m. & 74.979 & $\mathrm{~b}$ \\
ald1 P.s.m. & 51.0686 & $\mathrm{~b}$ \\
ald1 mock & 40.70441667 & $\mathrm{~b}$ \\
sard4-5 mock & 39.06668333 & $\mathrm{~b}$ \\
\hline
\end{tabular}


ANOVA for Figure 4B

\begin{tabular}{lccccc}
\hline & df & SS & MS & F-value & P-value \\
\hline genotype & 2 & $11,752.47$ & $5,876.24$ & 10.6571 & 0.0003 \\
treatment & 1 & $5,876.24$ & $5,876.24$ & 8.2997 & 0.0068 \\
genotype $\times$ treatment & 5 & $29,381.18$ & $5,876.24$ & 310.7781 & $1.11 \mathrm{E}-16$ \\
residuals & 30 & $76,860.13$ & $2,562.00$ & & \\
\hline
\end{tabular}

ANOVA for Figure 5A

\begin{tabular}{lccccc}
\hline & df & SS & MS & F-value & P-value \\
\hline genotype & 3 & 0.3484 & 0.1161 & 3.7703 & 0.027 \\
treatment & 1 & 0.26 & 0.26 & 7.9508 & $1.00 \mathrm{E}-02$ \\
genotype $\times$ treatment & 7 & 0.82 & 0.12 & 119.3415 & $1.32 \mathrm{E}-12$ \\
residuals & 16 & 0.0157 & 0.001 & & \\
\hline
\end{tabular}

\section{ANOVA for Figure 5B}

\begin{tabular}{lccccc}
\hline & df & SS & MS & F-value & P-value \\
\hline genotype & 3 & 809.6088 & 269.8696 & 2.0668 & 0.1368 \\
treatment & 1 & $1,729.02$ & $1,729.02$ & 22.4808 & $9.87 \mathrm{E}-05$ \\
genotype $\times$ treatment & 7 & $3,326.07$ & 475.15 & 80.0401 & $2.95 \mathrm{E}-11$ \\
residuals & 16 & 94.983 & 5.9364 & & \\
\hline
\end{tabular}

Tukey's HSD groups for Figure 4B

\begin{tabular}{lcc}
\hline genotype $\times$ treatment & mean & group \\
\hline sard4-5 P.s.m. & 76.65661667 & $\mathrm{a}$ \\
sard4-5 mock & 0 & $\mathrm{~b}$ \\
WT mock & 0 & $\mathrm{~b}$ \\
WT P.s.m. & 0 & $\mathrm{~b}$ \\
ald1 mock & 0 & $\mathrm{~b}$ \\
ald1 P.s.m. & 0 & $\mathrm{~b}$ \\
\hline
\end{tabular}

\section{Tukey's HSD groups for Figure 5A}

\begin{tabular}{lcc}
\hline genotype $\times$ treatment & mean & group \\
\hline WT P.s.m. & 0.5886 & $\mathrm{a}$ \\
fmo1 P.s.m. & 0.2376 & $\mathrm{~b}$ \\
WT mock & 0 & $\mathrm{C}$ \\
sard4-5 mock & 0 & $\mathrm{C}$ \\
fmo1 mock & 0 & $\mathrm{C}$ \\
ald1 mock & 0 & $\mathrm{C}$ \\
sard4-5 P.s.m & 0 & $\mathrm{C}$ \\
ald1 P.s.m. & 0 & $\mathrm{C}$ \\
\hline
\end{tabular}

Tukey's HSD groups for Figure 5B

\begin{tabular}{lcc}
\hline genotype $\times$ treatment & mean & group \\
\hline fmo1 P.s.m. & 30.2538 & $\mathrm{a}$ \\
WT P.s.m. & 24.5918 & $\mathrm{a}$ \\
sard4-5 P.s.m & 13.7923 & $\mathrm{~b}$ \\
WT mock & 0.3667 & $\mathrm{C}$ \\
sard4-5 mock & 0.1743 & $\mathrm{C}$ \\
fmo1 mock & 0.1649 & $\mathrm{C}$ \\
ald1 mock & 0.0352 & $\mathrm{C}$ \\
ald1 P.s.m. & 0.0055 & $\mathrm{C}$ \\
\hline
\end{tabular}


ANOVA for Figure 9A

\begin{tabular}{lccccc}
\hline & df & SS & MS & F-value & P-value \\
\hline genotype $\times$ treatment & 4 & 39.533 & 9.8832 & 196.37 & $1.86 \mathrm{E}-09$ \\
residuals & 10 & 0.5033 & 0.0503 & & \\
\hline
\end{tabular}

Tukey's unequal N HSD groups for Figure 9A

\begin{tabular}{lcc}
\hline genotype $\times$ treatment & mean & group \\
\hline FMO1-3D & 3.34466 & $\mathrm{a}$ \\
sard4-4 FMO1-3D Pip & 3.307500333 & $\mathrm{a}$ \\
sard4-4 FMO1-3D & 0.028772 & $\mathrm{~b}$ \\
WT Pip & 0.005109 & $\mathrm{~b}$ \\
WT & 0.003324333 & $\mathrm{~b}$ \\
\hline
\end{tabular}

ANOVA for Figure 9B

\begin{tabular}{lccccc}
\hline & df & SS & MS & F-value & P-value \\
\hline genotype $\times$ treatment & 4 & 0.0375 & 0.0094 & 78.5793 & $1.63 \mathrm{E}-07$ \\
residuals & 10 & 0.0012 & 0.0001 & & \\
\hline
\end{tabular}

Tukey's unequal N HSD groups for Figure 9B

\begin{tabular}{lcc}
\hline genotype $\times$ treatment & mean & group \\
\hline FMO1-3D & 0.110937333 & $\mathrm{a}$ \\
sard4-4 FMO1-3D Pip & 0.097814 & $\mathrm{a}$ \\
sard4-4 FMO1-3D & 0.005183 & $\mathrm{~b}$ \\
WT Pip & 0.001863667 & $\mathrm{~b}$ \\
WT & 0.000936 & $\mathrm{~b}$ \\
\hline
\end{tabular}

ANOVA for Figure 10A

\begin{tabular}{cccccc}
\hline & df & SS & MS & F-value & P-value \\
\hline genotype & 2 & 3.612 & 1.806 & 514.08 & $1.95 \mathrm{E}-07$ \\
residuals & 6 & 0.021 & 0.004 & & \\
\hline
\end{tabular}

Tukey's unequal N HSD groups for Figure 10A

\begin{tabular}{lcc}
\hline genotype & mean & group \\
\hline FMO1-3D & 1.3517 & $\mathrm{a}$ \\
WT & 0.0102 & $\mathrm{~b}$ \\
ald1 FMO1-3D & 0.0053 & $\mathrm{~b}$ \\
\hline
\end{tabular}


ANOVA for Figure 10B

\begin{tabular}{lccccc}
\hline & df & SS & MS & F-value & P-value \\
\hline genotype & 2 & 0.0072 & 0.0036 & 164.5865 & $5.74 \mathrm{E}-06$ \\
residuals & 6 & 0.0001 & 0 & & \\
\hline
\end{tabular}

ANOVA for Figure 10C

\begin{tabular}{cccccc}
\hline & df & SS & MS & F-value & P-value \\
\hline genotype & 2 & 43.1293 & 21.5647 & 69.1852 & $3.44 \mathrm{E}-06$ \\
residuals & 9 & 2.8053 & 0.3117 & & \\
\hline
\end{tabular}

Tukey's unequal N HSD groups for Figure 10C genotype

ald1 FMO1-3D

WT

mean

4.1858

3.8988

0.0284 group

a

b

b
FMO1-3D group

a

a

b

ANOVA for Supplemental Figure 1, Free SA

\begin{tabular}{lccccc}
\hline & df & SS & MS & F-value & P-value \\
\hline genotype & 1 & 0.01 & 0.01 & 0.0132 & 0.9096 \\
time & 2 & 12.36 & 6.18 & 278.3339 & $7.77 \mathrm{E}-16$ \\
genotype $\times$ time & 5 & 12.41 & 2.48 & 107.0406 & $9.64 \mathrm{E}-13$ \\
residuals & 18 & 0.42 & 0.02 & & \\
\hline
\end{tabular}

Tukey's HSD groups for Supplemental Figure 1, Free SA

\begin{tabular}{lcc}
\hline genotype $\times$ treatment & mean & group \\
\hline WT 12h & 1.790662581 & $\mathrm{a}$ \\
sard4-5 12h & 1.637603513 & $\mathrm{a}$ \\
WT 24h & 1.391942233 & $\mathrm{~b}$ \\
sard4-5 24h & 1.368012225 & $\mathrm{~b}$ \\
sard4-5 0h & 0.063219098 & $\mathrm{c}$ \\
WT 0h & 0.041491404 & $\mathrm{c}$ \\
\hline
\end{tabular}

ANOVA for Supplemental Figure 1, Total SA

\begin{tabular}{lccccc}
\hline & df & SS & MS & F-value & P-value \\
\hline genotype & 1 & 0.04 & 0.04 & 0.0005 & 0.9823 \\
time & 2 & $1,710.66$ & 855.3306 & 348.4211 & $1.11 \mathrm{E}-16$ \\
genotype $\times$ time & 5 & $1,714.07$ & 342.8144 & 128.177 & $2.01 \mathrm{E}-13$ \\
residuals & 18 & 48.1417 & 2.6745 & & \\
\hline
\end{tabular}


Tukey's HSD groups for Supplemental Figure 1, Free SA

\begin{tabular}{lcc}
\hline genotype $\times$ treatment & mean & group \\
\hline WT 24h & 20.26159737 & a \\
sard4-5 24h & 19.21905511 & a \\
sard4-5 12h & 4.160337996 & b \\
WT 12h & 3.373976411 & b \\
sard4-5 0h & 0.383617894 & $\mathrm{c}$ \\
WT 0h & 0.373710726 & $\mathrm{c}$ \\
\hline
\end{tabular}

ANOVA for Supplemental Figure 2A

\begin{tabular}{lccccc}
\hline & df & SS & MS & F-value & P-value \\
\hline genotype & 1 & 193.99 & 193.99 & 0.0164 & 0.8997 \\
time & 2 & $188,471.67$ & $94,235.83$ & $1,230.96$ & $1.11 \mathrm{E}-16$ \\
genotype $\times$ time & 5 & $188,903.20$ & $37,780.64$ & 632.4967 & $4.25 \mathrm{E}-14$ \\
residuals & 12 & 716.7906 & 59.7326 & & \\
\hline
\end{tabular}

Tukey's HSD groups for Supplemental Figure 2A

\begin{tabular}{lcc}
\hline genotype $\times$ treatment & mean & group \\
\hline WT 24h & 238.9839557 & a \\
sard4-5 24h & 222.2838741 & a \\
WT 12h & 31.78198147 & b \\
sard4-5 12h & 28.81623621 & b \\
WT 0h & 0.031478838 & c \\
sard4-5 0h & $9.78 \mathrm{E}-05$ & $\mathrm{c}$ \\
\hline
\end{tabular}

ANOVA for Supplemental Figure 2B

\begin{tabular}{lccccc}
\hline & df & SS & MS & F-value & P-value \\
\hline genotype & 1 & 0.0188 & 0.0188 & 0.0417 & 0.8409 \\
time & 2 & 7.17 & 3.59 & 813.73 & $5.55 \mathrm{E}-16$ \\
genotype $\times$ time & 5 & 7.2042 & 1.4408 & 486.056 & $2.05 \mathrm{E}-13$ \\
residuals & 12 & 0.0356 & 0.003 & & \\
\hline
\end{tabular}




\section{Tukey's HSD groups for Supplemental Figure 2B}

\begin{tabular}{lcc} 
genotype $\times$ treatment & mean & group \\
\hline sard4-5 12h & 1.571097698 & $\mathrm{a}$ \\
WT 12h & 1.503378138 & $\mathrm{a}$ \\
sard4-5 24h & 0.671415652 & $\mathrm{~b}$ \\
WT 24h & 0.545805826 & $\mathrm{~b}$ \\
sard4-5 0h & 0.00239703 & $\mathrm{c}$ \\
WT 0h & $1.82 \mathrm{E}-03$ & $\mathrm{c}$ \\
\hline
\end{tabular}

ANOVA for Supplemental Figure 3

\begin{tabular}{lccccc}
\hline & df & SS & MS & F-value & P-value \\
\hline genotype & 2 & 53.7031 & 26.8515 & $1,822.01$ & $1.11 \mathrm{E}-16$ \\
residuals & 15 & 0.2211 & 0.0147 & & \\
\hline
\end{tabular}

Tukey's unequal N HSD groups for Supplemental Figure 3

\begin{tabular}{lcc}
\hline genotype & mean & group \\
\hline npr1-1 & 9.1647 & $\mathrm{a}$ \\
sard4-5 & 5.5125 & $\mathrm{~b}$ \\
WT & 5.4888 & $\mathrm{~b}$ \\
\hline
\end{tabular}


Characterization of a Pipecolic Acid Biosynthesis Pathway Required for Systemic Acquired Resistance

Pingtao Ding, Dmitrij Rekhter, Yuli Ding, Kirstin Feussner, Lucas Busta, Sven Haroth, Shaohua Xu, Xin Li, Reinhard Jetter, Ivo Feussner and Yuelin Zhang

Plant Cell 2016;28;2603-2615; originally published online October 6, 2016;

DOI 10.1105/tpc.16.00486

This information is current as of November 13, 2016

Supplemental Data

References

Permissions

eTOCs

CiteTrack Alerts

Subscription Information
http://www.plantcell.org/content/suppl/2016/10/06/tpc.16.00486.DC1.html

This article cites 32 articles, 13 of which can be accessed free at: http://www.plantcell.org/content/28/10/2603.full.html\#ref-list-1

https://www.copyright.com/ccc/openurl.do?sid=pd_hw1532298X\&issn=1532298X\&WT.mc_id=pd_hw1532298X

Sign up for eTOCs at:

http://www.plantcell.org/cgi/alerts/ctmain

Sign up for CiteTrack Alerts at:

http://www.plantcell.org/cgi/alerts/ctmain

Subscription Information for The Plant Cell and Plant Physiology is available at: http://www.aspb.org/publications/subscriptions.cfm 


\section{Article II. From isochorismate to salicylate: a new reaction mechanism for salicylic acid biosynthesis}

The article was submitted in November 2018. The supplementary materials are attached to the main part.

\section{Author contribution:}

Dmitrij Rekhter planned and performed the metabolite fingerprint analysis as well as the subsequent metabolite identification by high resolution UHPLC-Q-TOF-MS/MS analysis. Moreover, he amplified, cloned, expressed and established the purification of AtICS1 and AtPBS3. He performed all in vitro studies with the purified enzymes. He created the ICS1CFP, PBS3-YFP and chloroPBS3-YFP construct and performed with these constructs the transient expression. He performed the in silico docking study and the molecular modeling of the non-enzymatic decay. He analyzed, processed, displayed, interpreted and discussed the results, and wrote the first draft of the manuscript. 


\section{From isochorismate to salicylate: a new reaction mechanism for salicylic acid biosynthesis}

Dmitrij Rekhter ${ }^{1}$, Yuli Ding ${ }^{2}$, Daniel Lüdke ${ }^{3}$, Kirstin Feussner ${ }^{1,4}$, Marcel Wiermer ${ }^{3}$, Yuelin Zhang $^{2, *}$, Ivo Feussner ${ }^{1,5, *}$

${ }^{1}$ University of Goettingen, Albrecht-von-Haller-Institute for Plant Sciences, Department of Plant Biochemistry, D-37077 Goettingen, Germany.

${ }^{2}$ University of British Columbia, Department of Botany, Vancouver, BC V6T 1Z4, Canada.

${ }^{3}$ University of Goettingen, Albrecht-von-Haller-Institute for Plant Sciences, RG Molecular Biology of Plant-Microbe Interactions, D-3707 Goettingen, Germany.

${ }^{4}$ University of Goettingen, Goettingen Center for Molecular Biosciences (GZMB), Service Unit for Metabolomics and Lipidomics, D-37077 Goettingen, Germany.

${ }^{5}$ University of Goettingen, Goettingen Center for Molecular Biosciences (GZMB), Department of Plant Biochemistry, D-37077 Goettingen, Germany.

*Correspondence to Ivo Feussner: ifeussn@uni-goettingen.de or Yuelin Zhang: yuelin.zhang@ubc.ca.

Abstract: Salicylic acid (SA) is an essential regulator of plant immune responses. Despite decades of research, our knowledge of its biosynthesis remains incomplete. Here we report that avrPphB Susceptible 3 (PBS3) catalyzes the formation of Isochorismate-9-glutamate (ISC-9-Glu) by conjugating glutamate to isochorismic acid and ISC-9-Glu further decays into SA without the requirement of any enzymatic catalysis. Thus, plant SA biosynthesis can be reconstituted with just the two enzymes Isochorismate Synthase 1 (ICS1) and PBS3 along with the putative isochroristmate transporter Enhanced Disease Susceptibility 5 (EDS5). This is the first example of decay driven biosynthesis of a phytohormone known so far.

One Sentence Summary: PBS3 produces SA via Isochorismate-9-glutamate.

Main Text: Salicylic acid (SA) is a plant defense hormone with central functions in regulating plant immune responses (1). The crucial role of SA as the modulator of local as well as systemic immune response has been studied extensively (2). Early $\left[{ }^{14} \mathrm{C}\right]$-feeding experiments suggested that SA is synthesized from phenylalanine via cinnamic acid $(3,4)$. However, the discovery of Isochorismate Synthase 1 (ICS1) in Arabidopsis thaliana showed that $\sim 90 \%$ of the pathogen-induced SA is produced via isochorismic acid (ISC) $(5,6)$. SA was proposed to be synthesized in plastids and exported to the cytosol by Enhanced Disease Susceptibility 5 (EDS5), which is a member of the MATE transporter family (7). This ICSdependent pathway is known from bacteria, where chorismic acid (CA) is either converted by a bifunctional SA synthase (SAS) to SA or by two separate enzymes, whereby CA is isomerized to ISC first, followed by a cleavage through an isochorismate pyruvate lyase (IPL). However, Arabidopsis ICS1 was reported to be a monofunctional enzyme and no IPL could be found in plant genomes (8). Therefore, it is unclear how ISC is converted to SA in higher plants. 
In Arabidopsis pbs3 mutant plants, the accumulation of SA and SA-glycoside (SAG) upon Pseudomonas syringe infection is reduced, but not abolished (9). PBS3 encodes an amido transferase of the GH3 family. The founding member of this protein family, AtGH3.11 (JAR1), catalyzes the conjugation of isoleucine to jasmonic acid (JA) to form the hormone conjugate JA-isoleucine (JA-Ile), which represents the active form of the JA signaling (10). However, SA was shown to be a poor substrate for PBS3 and even an inhibitor at higher concentrations (11). The function of PBS3 remains to be determined.

To better understand the role of PBS3 in plant defense, we crossed pbs3-1 into the autoimmune mutant snc2-1D npr1-1 (12), and performed metabolite fingerprinting analysis. The previously reported snc2-1D npr1-1 eds5-3 triple mutant (12) was used as a control. Similar to eds5-3, pbs3-1 cannot suppress the dwarf phenotype of snc2-1D npr1-1 (Fig. 1A).

In snc2-1D npr1-1, SA (Fig. 1B), SAG (Fig. 1C) and the SA catabolites 2,3- as well as 2,5dihydroxybenzoic acid xyloside (Fig. 1D and S1) accumulate drastically (13). The loss-offunction mutation of either PBS3 or EDS5 leads to dramatically reduced accumulation of SA and its derivatives. Interestingly, we could detect a significant accumulation of the SA precursor ISC in the snc2-1D npr1-1 pbs3-1 but not in the snc2-1D npr1-1 eds5-3 mutant (Fig. 1E). Based on these findings, we hypothesized that ISC could be the in planta substrate for PBS3.

To test our hypothesis, we expressed poly-histidine tagged PBS3 in Escherichia coli (E. coli) and purified it to homogeneity (Fig. S2). First, we tested whether PBS3 is able to utilize CA for the conjugation with glutamic acid (Glu) (11) by monitoring the production of CA-Glu. Interestingly, the reaction yields two signals for the enzymatic product CA-Glu $(\mathrm{m} / \mathrm{z} 354.083$, Fig. 2A red graph). The MS/MS fragmentation analysis showed that both the $\mathrm{C} 7$ and the $\mathrm{C} 9$ carboxyl group are targets for the amido transferase (CA-7-Glu, CA-9-Glu, Fig.2B a, d, Fig. S3). Next, we tested whether PBS3 is also able to use ISC as a substrate. As ISC is not commercially available, we purified heterologously expressed ICS1 to homogeneity and used it to produce ISC from CA (Fig. S4 and S5). We removed the active ICS1 from the reaction by filter centrifugation. The ISC along with residual amounts of CA in the reaction was used as substrate for PBS3. The PBS3 activity assay yields four signals of $m / z$ 354.083, thereby two represent the CA-conjugates (Fig. 2A a and d). The most intense signal (at $2.85 \mathrm{~min}$ ) was unequivocally identified via MS/MS fragmentation as ISC-9-Glu (Fig. 2A c, 2B c, S3). The structures for the four signals are depicted in Fig. 2B. With the assumption that these four compounds have similar ionization properties, we conclude that the formation of ISC-9-Glu (Fig. 2B c) is the preferred reaction of PBS3.

Remarkably, we could detect the presence of SA in the PBS3 activity assay (Fig. 2C). It is known that ISC itself decays slowly to SA in solution (14). Therefore, we tested if the formation of SA relies on the completeness of the activity assay by leaving out individual components of the PBS3 reaction. Indeed, we could detect a small amount of SA in the absence of either Glu, ATP or PBS3 in the enzymatic assay. However, within the first hour the SA signal was four times higher in the full reaction than in the controls. As no lyase activity has been reported for GH3 proteins so far, most likely the formation of ISC-9-Glu strongly enhances the decay of ISC, leading to the accumulation of SA.

The activity of GH3 enzymes consists of a two-step reaction. In the first step, an acyl substrate is adenylated and pyrophosphate is released. An amino acid is conjugated to the activated acyl substrate in the second step. Thereby, the resulting acyl-amino acid conjugate as well as adenosine monophosphate (AMP) are released. The kinetic parameters for the PBS3 catalyzed conjugation of Glu with ICS were determined by a spectrophotometric assay (Fig. 2D) (15). The previously investigated acyl substrate 4-hydroxybenzoic acid (4HBA) 
was used as a control (11). Strikingly, we had to dilute the enzyme one hundred fold when we used ISC as acyl substrate instead of 4HBA, as the reaction was otherwise too fast for an accurate measurement. The catalytic efficiency $\left(\mathrm{k}_{\mathrm{cat}} / \mathrm{K}_{\mathrm{m}}\right)$ of PBS3 was increased by a factor of $\sim 740$ when ISC $\left(31.70 \mathrm{~min}^{-1} \mu \mathrm{M}^{-1}\right)$ was used as the acyl substrate in place of 4HBA $\left(0.043 \mathrm{~min}^{-1} \mu \mathrm{M}^{-1}\right)$. This increase is a result of a much higher substrate affinity ( 21 -fold) and an enhanced turnover rate ( $\sim 35$-fold) for ISC. These data suggest that ISC is a preferred substrate of PBS3. Next we utilized the publically available crystal structure of PBS3, which was co-crystallized with SA and AMP (PDB ID 4eql) (16), to model ISC into the active center (Fig. S6A). When we superimposed the ring-structure of SA with that of ISC (Fig. S6 B-D), we observed that i) there is space to accommodate the bulky side chain of the ISCmolecule in the active center and ii) the phosphate group of the AMP-moiety - and the C9carboxyl group of ISC are in close proximity ( 2.5 Å; Fig. S6C). Based on the high efficiency of PBS3 in catalyzing the conjugation of ISC to Glu and the geometry of the active site, we propose that PBS3 is directly involved in the biosynthesis of SA (Fig. 2E).

Beside SA, we also detected 2-hydroxy acryloyl-N-glutamic acid (2HNG, m/z 216.051), as the second product of the decay of ISC-9-Glu in the PBS3 in vitro assay. To determine whether the decay of ISC-9-Glu also occurs in vivo, we analyzed the accumulation of ISC-9Glu and 2NHG in planta. Both compounds were detected in snc2-1D npr1-1 samples, with corresponding retention times and mass per charge ratios as in the in vitro enzymatic assay. However, they were neither detected in snc2-1D npr1-1 pbs3-1 nor in snc2-1D npr1-1 eds5-3 triple mutant plants (Fig. 3A and B), which correlates with the accumulation of SA in Fig. 1A. We performed MS/MS fragmentation for ISC-9-Glu produced in vivo (Fig. 3C) or in vitro (Fig. 3D), respectively. The fragmentation patterns were almost identical and all recorded fragments could be interpreted unequivocally by their exact mass information (Fig. $3 \mathrm{E}$ and F). Based on these data, we conclude that ISC-9-Glu is indeed the in planta precursor of SA, which decays rapidly after the induction of the biosynthesis pathway (Fig. 2E).

We further monitored the non-enzymatic formation of SA originating from the decay of ISC9-Glu and ISC for $24 \mathrm{~h}$. Within the first $6 \mathrm{~h}$ after starting the reaction, ISC-9-Glu shows a linear dissociation rate, which is 10-times higher compared to ISC (Fig. 3G). After $20 \mathrm{~h}$, ISC-9-Glu reaches its dissociation equilibrium. At this point, the dissociation of ISC is still in a linear range. In order to understand the accelerated decay, we employed molecular modeling for ISC-9-Glu. The resulting structure (Fig. S7A) strongly suggests the formation of three hydrogen bonds: between the alpha and omega carboxyl group of the glutamyl moiety and the carboxyl - respectively hydroxyl group of the ISC ring system, as well as the amide hydrogen of the peptide bond and the oxygen of the ether bridge. The later hydrogen bond brings these two functional groups in close proximity and this distance is most likely sufficient to facilitate a hydrogen transfer. As a consequence of this protonation, we expect a base-initiated aromatization of the ring system, which is followed by an elimination of the E1 type that yields SA and 2HNG as the final products (Fig. S7B). The chemical formation of SA via the non-enzymatic decay may partially explain the delayed accumulation of SA (12 hpi) relative to the induction of the expression of ICS1, PBS3 and EDS5 (6 hpi) upon pathogen challenge $(17,18)$.

The biosynthesis of SA was proposed to occur in the plastid, as ICS1 was shown to localize to this subcellular compartement (6). Interestingly, sequence analysis with the in silico online tools TargetP (19) and Predotar (20) predicted PBS3 to be localized to the cytosol. This is consistent with studies of other GH3 enzymes $(21,22)$. We used Agrobacterium-mediated transient expression of PBS3-YFP in Nicotiana benthamiana as well as in Arabidopsis efr mutant leaves (23). As predicted, the PBS3-YFP fluorescence signal could be detected mostly in the cytosol (Fig. 4A) using confocal laser scanning microscopy (CLSM). Utilizing the 
same construct, we were able to restore SA accumulation after transient expression in Arabidopsis pbs3 mutant plants (Fig. S8). To investigate the biochemical relationship of ICS1 and PBS3 further, we fused the transit peptide of ICS1 (6) to the N-terminus of PBS3 to obtain a chloroPBS3-YFP construct. When ICS1-CFP and chloroPBS3-YFP were transiently co-expressed in Arabidopsis efr mutant leaves, that allow enhanced Agrobacterium-mediated transient transformation rates (23), they were observed to co-localize in chloroplasts (Fig. 4B). We therefore utilized the fusion protein constructs expressing these two fusion proteins to monitor the SA formation in Arabidopsis eds5-3 mutant plants. Strikingly, transient expression of ICS1 together with chloroPBS3 restored SA accumulation. In contrast, the native PBS3 alone or in combination with ICS1 could not restore SA accumulation in eds5-3 plants (Fig. 4C). This strongly suggests that EDS5 is responsible for the export of ICS1derived ISC from the plastidial stroma into the cytosol, where it is further utilized by PBS3 (Fig. 4D). This would also explains why SA does not accumulate in eds5 mutants (Fig. 1A) (7). The need of ICS1 to restore SA accumulation in combination with chloroPBS3, could be explained by the inhibitory properties of SA towards PBS3 (11). As shown in the crystal structure of PBS3 (16), SA binds to the same pocket as ISC (Fig. S6). In order to overcome the competitive inhibition, transiently expressed ICS1 is required to produce sufficient amounts of ISC, which subsequently can be utilized by PBS3. Taken together, these data reveal not only how, but also where, SA is synthesized at the subcellular level.

By studying SA formation in the autoimmune mutant snc2-1D npr1-1 pbs3-1 we were able to identify ISC as the substrate for PBS3 (Fig. 1E). Heterologous expression and purification of PBS3 and ICS1 enabled us to study PBS3 in vitro. Thereby we found that PBS3 utilizes ISC as a substrate to conjugate it with glutamate to yield ISC-9-Glu (Fig. 2A). Kinetic analyses (Fig. 2D) as well as in silico studies (Fig. S6) confirmed the preference of PBS3 towards ISC as its native substrate. We further observed that the PBS3 derived ISC-9-Glu decays ten times faster than its precursor ISC in an enzyme independent manner (Fig. 3G) releasing SA and 2HNG as a result. Supporting our in vitro findings, we were able to detect ISC-9-Glu as well as 2HNG in planta (Fig. 3A and B). Finally, we could show why ICS1, EDS5 and PBS3 are all required for pathogen induced SA formation (Fig. 4B). While plastidial ICS1 is converting CA into ISC, EDS5 is required to transport ISC from the plastid into the cytosol, where it is utilized by PBS3 to yield ISC-9-Glu.

An overwhelming number of studies showed the central role of SA for plant immunity. Despite extensive efforts to decipher the biosynthesis of SA, it was not possible so far to produce SA with plant enzymes only. Our study now reveals the functions of EDS5 and PBS3 in the SA biosynthesis and completes the pathogen-induced SA biosynthesis pathway in Arabidopsis (Fig. 4C). Additionally, it explains the lack of IPLs in plant genomes. As PBS3 homologues are widespread throughout the plant kingdom (24), what we uncovered here (Fig. 4D) most likely represents the general principle for the biosynthesis of SA in higher plants. In addition, our study also uncovered a novel principle for the biosynthesis of a phytohormone. The enzymatically catalyzed formation of SA by pyruvate lyase from either CM or ISC in bacteria was proposed to follow a general base mechanism (25). The formation of ISC-9-Glu in higher plants directly brings an intramolecular base in close proximity to the ether oxygen, which enables the non-enzymatic heterolytic $\mathrm{C}-\mathrm{O}$ cleavage. These two residues are brought in close proximity by two additional hydrogen bonds formed by the two carboxyl groups of the glutamyl moiety, which keep the side chain of ISC-9-Glu close to the ring structure. Similar conformation was previously shown to enhance the lyase activity of an bacterial IPL enzyme (26). It strongly suggests that ISC-9-Glu automatically forms a transition state that is otherwise found in the active site of bacterial IPLs. This non-enzymatic final step in the production of a key regulatory compound like SA has the advantage that a 
decay process is uni-directional and thus unspecific side reactions, which are typical for IPL enzymes (27), are highly unlikely (28). In bacteria, SA occurs as an intermediate in siderophore biosynthesis (25). These iron chelators are essential for the survival and pathogenicity of the microbes. It is tempting to speculate that the reaction mechanism found here, occurs in pathogens also and thus would be a potential new target for antibiotics (Lamb 2015).

\section{References:}

1. $\quad$ D. M. A. Dempsey, D. F. Klessig, Trends Plant Sci. 17, 538 (2012).

2. $\quad$ I. A. Vos, C. M. J. Pieterse, S. C. M. van Wees, Plant Pathology 62, 43 (2013).

3. S. Z. El-Basyouni, D. Chen, R. Ibrahim, A. Neish, G. Towers, Phytochemistry 3, 485 (1964).

4. $\quad$ H. Klämbt, Nature 196, 491 (1962).

5. $\quad$ M. C. Wildermuth, J. Dewdney, G. Wu, F. M. Ausubel, Nature 414, 562 (2001).

6. $\quad$ C. Garcion et al., Plant Physiol. 147, 1279 (2008).

7. $\quad$ M. Serrano et al., Plant Physiol. 162, 1815 (2013).

8. D. M. A. Dempsey, A. C. Vlot, M. C. Wildermuth, D. F. Klessig, The Arabidopsis Book, e0156 (2011).

9. $\quad$ G. Jagadeeswaran et al., Plant J. 51, 234 (2007).

10. P. E. Staswick, I. Tiryaki, M. L. Rowe, Plant Cell 14, 1405 (2002).

11. R. A. Okrent, M. D. Brooks, M. C. Wildermuth, J. Biol. Chem. 284, 9742 (2009).

12. $\quad$ Y. Zhang et al., Plant Cell 22, 3153 (2010).

13. M. Bartsch et al., J. Biol. Chem. 285, 25654 (2010).

14. M. S. DeClue, K. K. Baldridge, P. Kast, D. Hilvert, J. Am. Chem. Soc. 128, 2043 (2006).

15. Q. Chen, C. S. Westfall, L. M. Hicks, S. Wang, J. M. Jez, J. Biol. Chem. 285, 29780 (2010).

16. C. S. Westfall et al., Science 336, 1708 (2012).

17. C. Nawrath, Plant Cell 14, 275 (2002).

18. K. Nobuta et al., Plant Physiol. 144, 1144 (2007).

19. O. Emanuelsson, H. Nielsen, S. Brunak, G. Von Heijne, J. Mol. Biol. 300, 1005 (2000).

20. I. Small, N. Peeters, F. Legeai, C. Lurin, Proteomics 4, 1581 (2004).

21. J. Ludwig-Müller, S. Jülke, N. M. Bierfreund, E. L. Decker, R. Reski, New Phytol. 181, 323 (2009).

22. M. Ostrowski, M. Świdziński, A. Ciarkowska, A. Jakubowska, Acta Physiol. Plant. 36, 3029 (2014).

23. C. Zipfel et al., Cell 125, 749 (2006).

24. R. A. Okrent, M. C. Wildermuth, Plant Mol. Biol. 76, 489 (2011).

25. C. T. Walsh, J. Liu, F. Rusnak, M. Sakaitani, Chemical Reviews 90, 1105 (1990).

26. S. Martí et al., J. Am. Chem. Soc. 131, 16156 (2009).

27. Q. Luo, K. M. Meneely, A. L. Lamb, J. Am. Chem. Soc. 133, 7229 (2011).

28. M. A. Keller, G. Piedrafita, M. Ralser, Curr. Opin. Biotechnol. 34, 153 (2015).

Acknowledgments: We are grateful to Volker Lipka and Elena Petutschnig (University of Goettingen) for providing infrastructure and support for confocal microscopy (Deutsche Forschungsgemeinschaft (DFG) INST 186/1277-1 FUGG), to Jane Parker (MPIPZ Cologne) for pXCSG-YFP/CFP destination vectors, to Ellen Hornung (University of Goettingen) for 
pEntry-C-eYFR vector, and to Egon Fanghänel for advises on chemical decay. Funding: This research has been funded by the DFG (IRTG 2172 "PRoTECT" program of the Göttingen Graduate Center of Neurosciences, Biophysics, and Molecular Biosciences.) to D.R., Y.D., D.L., M.W., Y.Z., and I.F.; I.F. was additionally supported by DFG excellence initiative (ZUK 45/2010). Y.Z. was supported by Natural Sciences and Engineering Research Council of Canada, Canada Foundation for Innovation, and British Columbia Knowledge Development Fund. Author contributions: D.R., Y.D., D.L. K.F., Y.Z., and I.F. conceived and designed the experiments. D.R., Y.D. and L.D. performed the experiments. D.R., Y.D., D.L., K.F., Y.Z., and I.F. analyzed the data, D.R., D.L, K.F., M.W., Y.Z., and I.F. wrote the article. Competing interests: Authors declare no competing interests. Data and materials availability: All data is available in the main text or the supplementary materials. The authors responsible for distribution of materials integral to the findings presented in this article are: Ivo Feussner (ifeussn@uni-goettingen.de) and Yuelin Zhang (yuelin.zhang@ubc.ca).

\section{Supplementary Materials:}

Materials and Methods

Figures S1-S8

Tables S1-S2

References (1-8) 
A

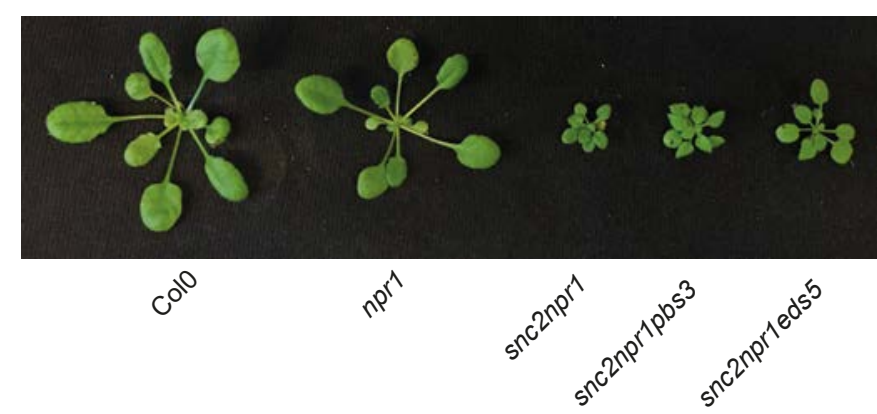

B

C
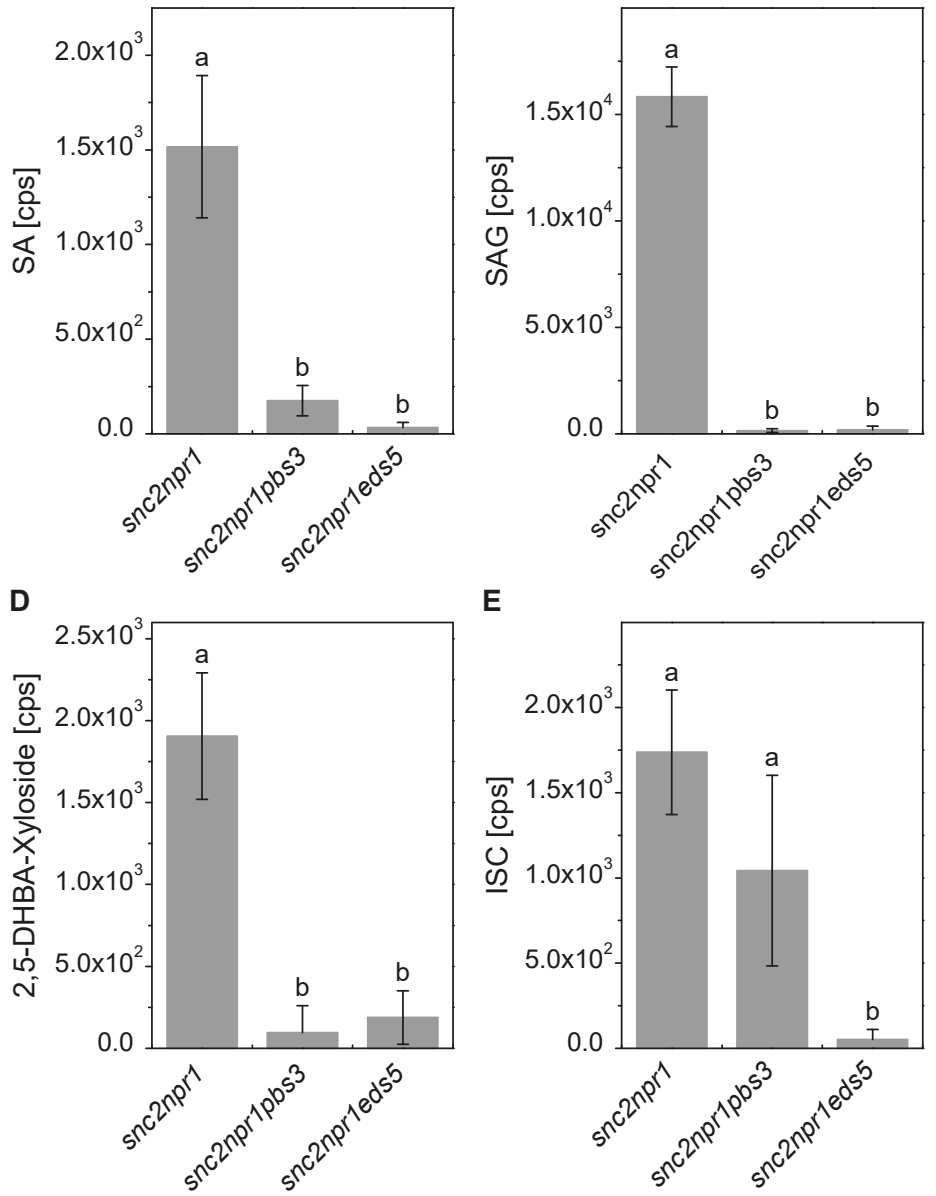

E

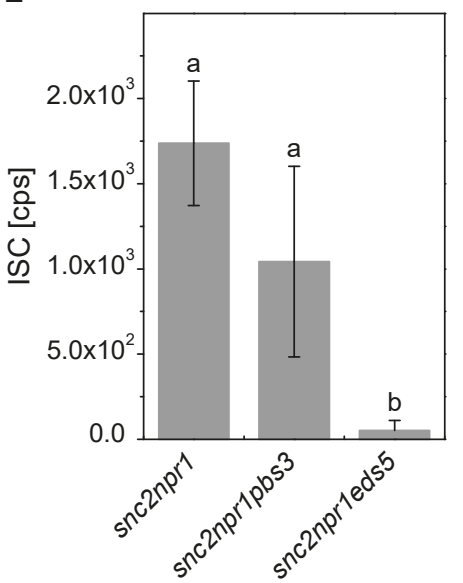

Fig. 1. Analysis of SA-related metabolites in snc2-1D npr1-1, snc2-1D npr1-1 pbs3-1 and snc2-1D npr 1-1 eds5-3. (A) Morphology of four-week-old wild-type (Col-0), npr 1-1, snc2$1 D$ nprl-1 (snc2 npr1), snc2-1D nprl-1 pbs3-1 (snc2 npr1 pbs3) and snc2-1D npr1-1 eds5-3 (snc2 nprleds5) plants. (B-E) Accumulation of SA (B), SAG (C), 2,5-DHBA-Xyloside (D) and ISC (E) in snc2 npr1, snc2-1 npr1 pbs3 and snc2 npr eds5. Relative compound amount (counts per second [cps]) were determined by LC-MS analysis of Arabidopsis thaliana leaf samples. Bars represent the mean \pm STD of three biological replicates. Statistical differences among replicates are labeled with different letters $(\mathrm{P}<0.05$, one-way ANOVA and post hoc Tukey's Test; $\mathrm{n}=3$ ). 
A

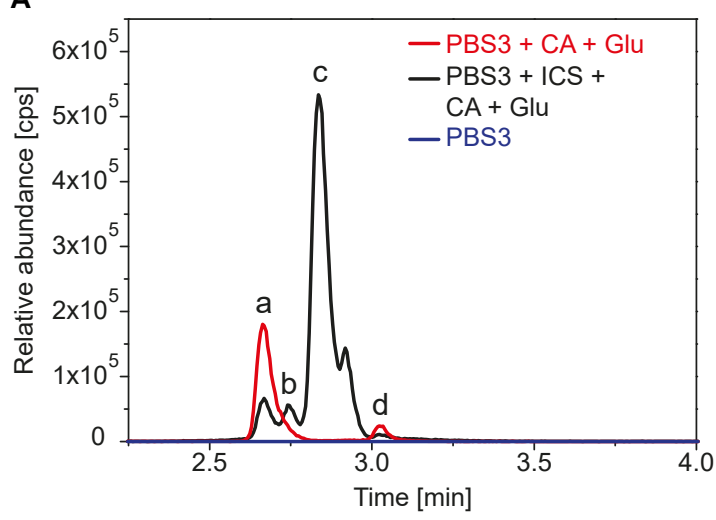

C

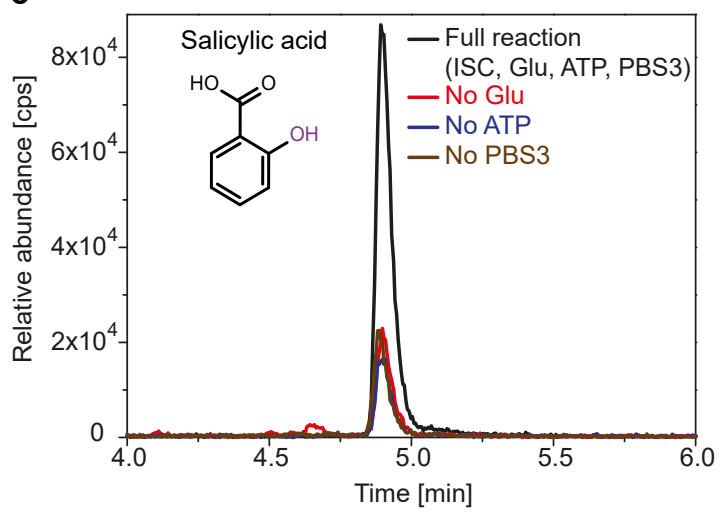

B<smiles>C=C(O)C(=O)OC1C=C(C(=O)NC(CCC(=O)O)C(=O)O)C=CC1O</smiles><smiles>C=C(NC(CCC(=O)O)C(=O)O)C(=O)OC1C=CC=C(C(=O)O)C1O</smiles>

D

\begin{tabular}{cccc}
\hline Substrate & $\mathbf{V} / \mathbf{E}_{\mathbf{t}}$ & $\mathbf{K}_{\mathbf{m}}$ & $\mathbf{K}_{\text {cat }} / \mathbf{K}_{\mathbf{m}}$ \\
\hline & {$\left[\mathrm{min}^{-1}\right]$} & {$[\mu \mathrm{M}]$} & {$\left[\mathrm{min}^{-1} \mu \mathrm{M}^{-1}\right]$} \\
4HBA & 13.46 & 311.54 & 0.043 \\
ISC & 472.29 & 14.90 & 31.70
\end{tabular}<smiles>C=C(OC1C=C(C(=O)O)C=CC1O)C(=O)NC(CCC(=O)O)C(=O)O</smiles><smiles>C=C(O)C(=O)OC1C=CC=C(C(=O)NC(CCC(=O)O)C(=O)O)C1O</smiles>

E

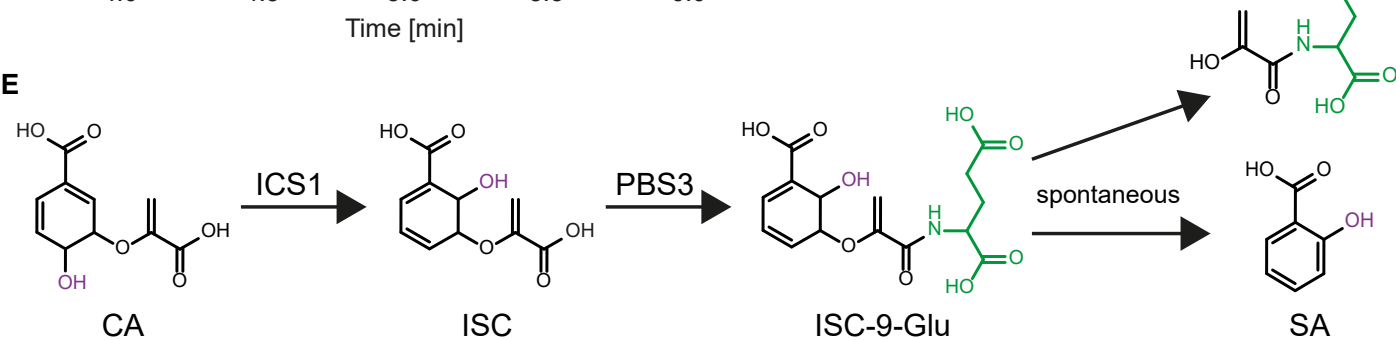

Fig. 2. PBS3 catalyzes the production of ISC-9-Glu and SA is formed from the decay of ISC-9-Glu. (A) LC-MS analysis of products from activity assays of purified PBS3. The assays were performed with chorismate (CA) and glutamate (Glu) (red line), with CA, Glu and Isochorismate synthase 1 (ICS1, which converts CA to isochorismate (ISC)) (black line) or without acyl substrate (blue line). The extracted ion chromatogram for ISC-9-Glu $(\mathrm{m} / \mathrm{z}$ 354.083) is shown. (B) Chemical structures of conjugates formed by PBS3 with Glu (green) and CA $(\mathrm{a}, \mathrm{d})$ or ISC $(\mathrm{b}, \mathrm{c})$. Depending on the acyl substrate, Glu is preferably conjugated to C7 (for CA) or C9 (for ISC). Structures were solved with MS/MS (Fig. S3). (C) Extracted ion chromatogram for SA $(\mathrm{m} / \mathrm{z} 137.024)$ which accumulates in the PBS3 activity assay with ISC, Glu and ATP (black line), but only in minor amount in the absence of Glu (red line), ATP (blue line) or PBS3 (brown line). The presence of SA in the control assays is due to ISC decay in solution. The identity of SA was confirmed by an authentical standard. (D) Kinetic parameters of PBS3 with 4HBA or ISC as acyl substrate. Data were obtained spectrophotometrically in triplicates. (E) Proposed SA biosynthesis pathway starting from CA. CA is converted by ICS1 to ISC, which is subsequently conjugated to Glu by PBS3. ISC-9-Glu decays spontaneously to SA and 2-hydroxyacryloyl-N-glutamic acid (2HNG). 
A

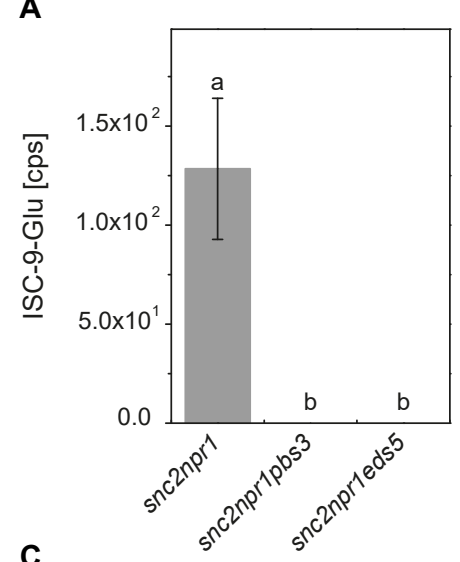

C

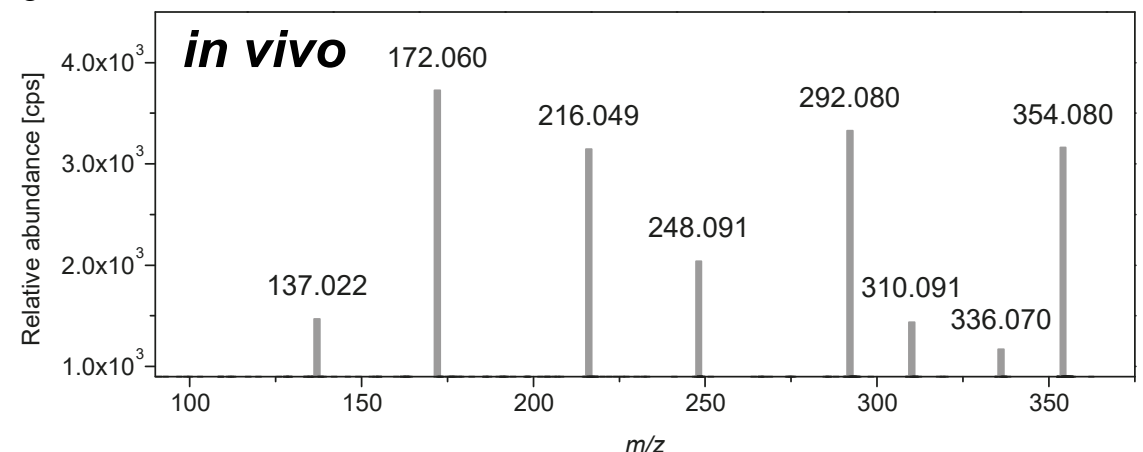

D

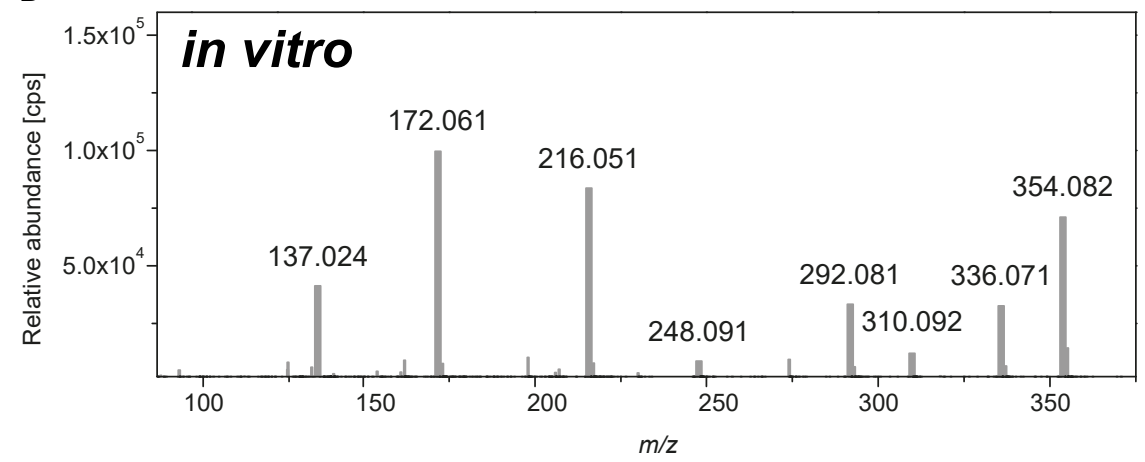

$E$

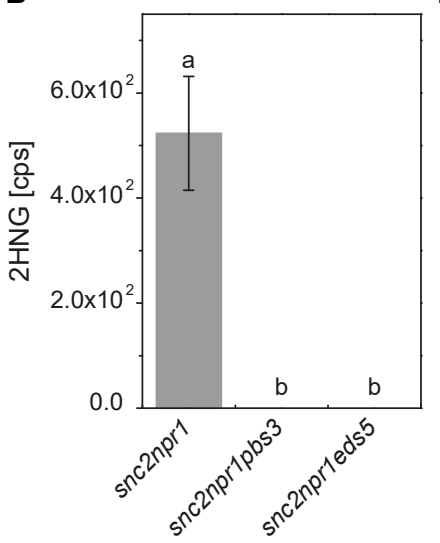

\begin{tabular}{|c|c|c|}
\hline $\begin{array}{c}M^{2} \text { fragment } \\
\text { of ISC-9-Glu }[\mathrm{m} / \mathrm{z}]\end{array}$ & $\begin{array}{c}\text { Elemental } \\
\text { composition }\end{array}$ & Identity \\
\hline 354.080 & $\mathrm{C}_{15} \mathrm{H}_{16} \mathrm{NO}_{9}$ & {$[\mathrm{M}-\mathrm{H}]^{-}$} \\
\hline 336.070 & $\mathrm{C}_{15} \mathrm{H}_{14} \mathrm{NO}_{8}$ & {$\left[\mathrm{M}-\mathrm{H}_{2} \mathrm{O}-\mathrm{H}\right]^{-}$} \\
\hline 310.091 & $\mathrm{C}_{14} \mathrm{H}_{16} \mathrm{NO}_{7}$ & {$\left[{\left.\mathrm{M}-\mathrm{CO}_{2}-\mathrm{H}\right]^{-}}^{-2}\right.$} \\
\hline 292.080 & $\mathrm{C}_{14} \mathrm{H}_{14} \mathrm{NO}_{6}$ & {$\left[\mathrm{M}-\mathrm{H}_{2} \mathrm{O}-\mathrm{CO}_{2}-\mathrm{H}\right]^{-}$} \\
\hline 248.091 & $\mathrm{C}_{13} \mathrm{H}_{14} \mathrm{NO}_{4}$ & {$\left[\mathrm{M}-\mathrm{H}_{2} \mathrm{O}-2 \mathrm{CO}_{2}-\mathrm{H}\right]^{-}$} \\
\hline 216.049 & $\mathrm{C}_{8} \mathrm{H}_{10} \mathrm{NO}_{6}$ & {$\left[\mathrm{M}-\mathrm{SA}-\mathrm{H}^{-}\right.$} \\
\hline 172.060 & $\mathrm{C}_{7} \mathrm{H}_{10} \mathrm{NO}_{4}$ & {$\left[\mathrm{M}-\mathrm{SA}-\mathrm{CO}_{2}-\mathrm{H}\right]^{-}$} \\
\hline 137.022 & $\mathrm{C}_{7} \mathrm{H}_{5} \mathrm{O}_{3}$ & {$[\mathrm{SA}-\mathrm{H}]^{-}$} \\
\hline & & \\
\hline
\end{tabular}

$\mathbf{F}$

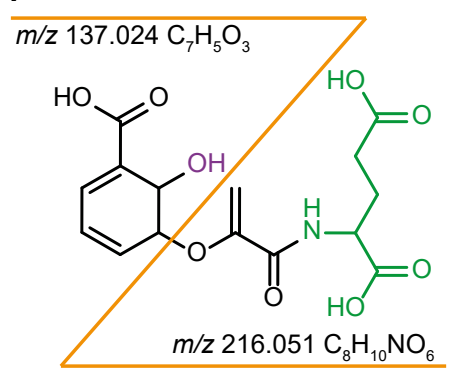

G

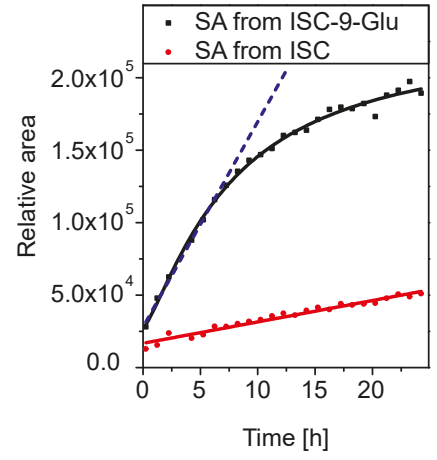

Fig. 3. PBS3-dependent production of ISC-9-Glu, 2NHG and SA in planta. (A, B) Accumulation of ISC-9-Glu (A) and 2-hydroxyacryloyl-N-glutamic acid (2HNG) (B) in snc2 $n p r 1$, snc 2 npr 1 pbs 3 and snc 2 npr 1 eds 5 plants. Bars represent the mean \pm STD of three biological replicates. Statistical differences among replicates are labeled with different letters $(\mathrm{P}<0.05$, one-way ANOVA and post hoc Tukey's Test; $\mathrm{n}=3)$. $(\mathbf{C}, \mathbf{D}) \mathrm{MS} / \mathrm{MS}$ fragmentation of ISC-9-Glu from snc2nprl plant material (C) and PBS3 activity assay (D). (E) Annotation list for MS/MS fragments of ISC-9-Glu from snc2nprl plant material. Fragments were identified by accurate mass analysis. (F) Proposed decay of ISC-9-Glu into SA and 2HNG. (G) Time course for the formation of SA from the decay of ISC (red line) and ISC-9-Glu (black line). 
A

ICS1-CFP and PBS3-YFP
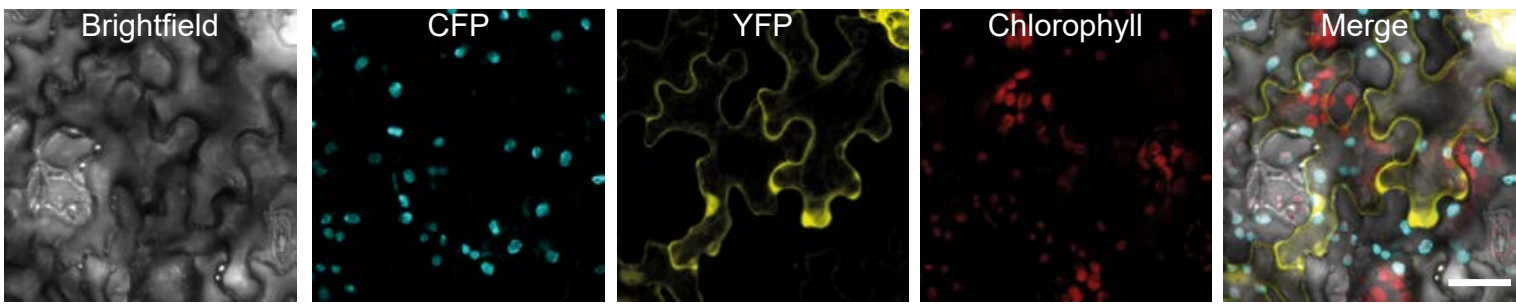

B

ICS1-CFP and chloroPBS3-YFP
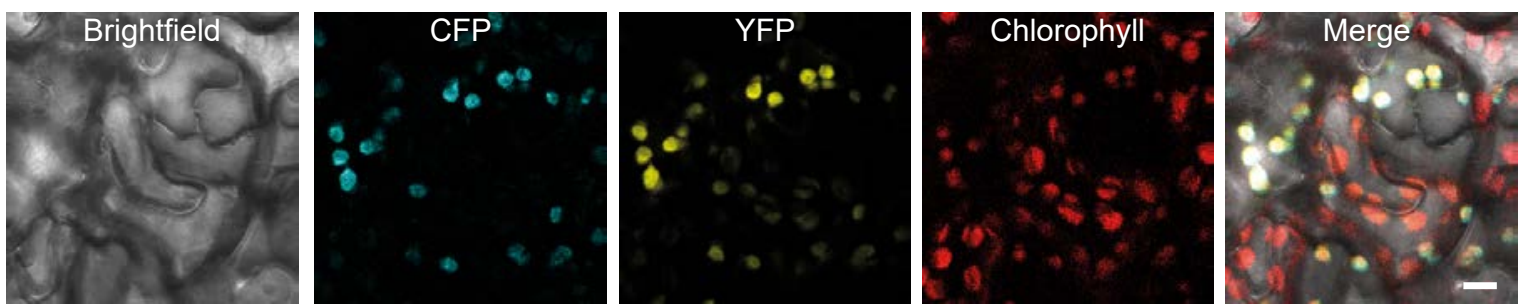

C

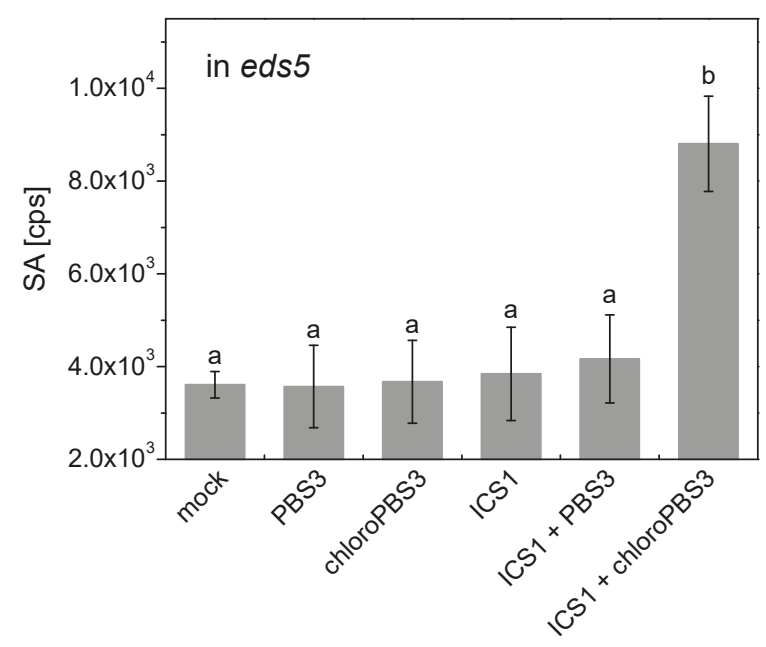

D

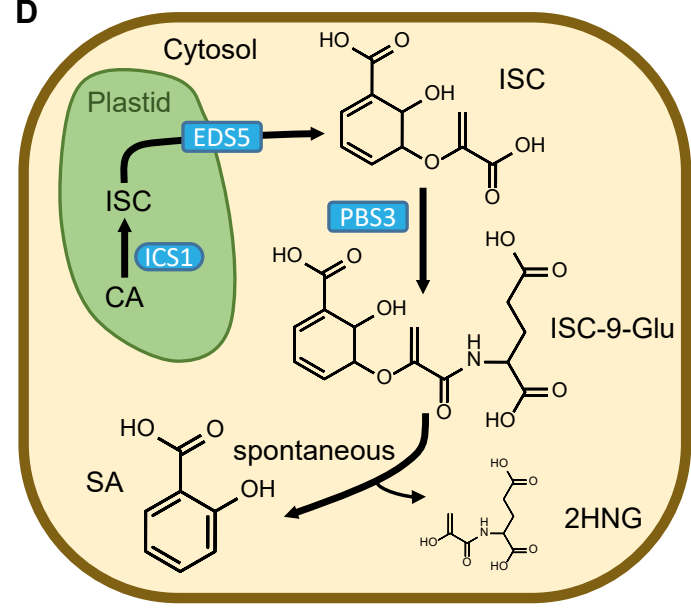

Fig. 4. Plastidial localized PBS3 and ICS1 are sufficient to produce SA in eds5 plants.

Confocal laser scanning microscopy analysis three days after Agrobacterium-mediated transient co-expression of (A) ICS1-CFP and PBS3-YFP respectively (B) ICS1-CFP and plastidial chloroPBS3-YFP in Arabidopsis efr leaves. Scale bar represents $10 \mu \mathrm{m}$. (C) Transient expression of ICS1 and chloroPBS3 in Arabidopsis eds5-3 leaves restores the Agrobacterium tumefaciens induced SA accumulation. $24 \mathrm{~h}$ after infiltration, leaves were collected and metabolites were extracted as described for Metabolite fingerprint analysis. Infiltration medium was used as mock treatment. The SA content was analyzed by UHPLCQ-TOF-MS. Bars represent the mean \pm STD of three biological replicates. Statistical differences among replicates are labeled with different letters $(\mathrm{P}<0.05$, one-way ANOVA and post hoc Tukey's Test; $n=3$ ). (C) A working model summarizing pathogen-induced SA biosynthesis in plants. 


\section{Supplementary Materials for}

From isochorismate to salicylic acid: a new reaction mechanism for salicylic acid biosynthesis

Dmitrij Rekhter, Yuli Ding, Daniel Lüdke, Kirstin Feussner, Marcel Wiermer, Yuelin Zhang $^{*}$, Ivo Feussner ${ }^{*}$

Correspondence to: Ivo Feussner: ifeussn@uni-goettingen.de or Yuelin Zhang: yuelin.zhang@ubc.ca

\section{This PDF file includes:}

Materials and Methods

Figs. S1-S8

Tables S1-S2

Reference List (1-8) 


\section{Materials and Methods}

Metabolite fingerprinting analysis

The non-targeted metabolite analysis was performed as described in $(1,2)$. In short, metabolites were extracted from $\sim 100 \mathrm{mg}$ leaf material or from two-week-old seedlings growing on tissue culture plates by two-phase extraction with methyl-tert-butylether (MTBE). Extracts were separated with an Ultra Performance Liquid Chromatography (UPLC, ACQUITY UPLC System; Waters Corporation, USA) equipped with an ACQUITY UPLC HSS T3 column ( 1.0 x $100 \mathrm{~mm}, 1.8 \mu \mathrm{m}$ particle size, Waters Corporation, USA), coupled to a time-of-flight mass spectrometer (TOF-MS, LCT Premier; Waters Corporation, USA).

For peak picking and alignment MarkerLynx Application Manager 4.1 for MassLynx software was used. For subsequent data processing, ranking, filtering, adduct correction, clustering and database analysis, MarVis software (MarkerVisualization, http://marvis.gobics.de, (2)) was utilized. Bars represent the mean \pm STD of three biological replicates. Statistical differences among replicates are labeled with different letters $(\mathrm{P}<0.05$, one-way ANOVA and post hoc Tukey’s Test; $n=3$ ). All experiments were repeated at least twice with similar results.

\section{High-resolution MS/MS analysis}

The chemical structures of CA, ISC, SA, 2HNG, CA-7-Glu, CA-9-Glu, ISC-7-Glu and ISC-9-Glu were elucidated by MS/MS analyses with an 1290 Infinity UHPLC system coupled to a 6540 UH AccurateMass-Q-TOF-MS (Agilent Technologies, USA) as previously described (1). Data acquisition was monitored with Mass Hunter Workstation Acquisition software B.05.01 (Agilent Technologies, USA). For data analysis, Mass Hunter Qualitative Analysis software B.05.01 was used (Agilent Technologies, USA). Authentic standards for SA (BioXtra, $\geq 99.0 \%$, S5922 Sigma, Germany) and CA ( $\geq 80 \%$, C1761 Aldrich, Germany) were obtained from Merck, Germany. All fragmentations were carried out in the negative ionization mode. The results are shown in Fig. 3C-E, S3, S5 and are summarized in Table S1.

\section{Protein expression and purification}

AtGH3.12 was amplified by PCR from A. thaliana ecotype Col0 cDNA derived from systemic leaves after infection (1). AtICS1 was amplified without the transit peptide from Col0 cDNA of infected leaves. AtGH3.12 was subcloned into pET24 (Novagen, Germany), AtICS1 into pET28a (Novagen, Germany). Both His-tagged proteins were expressed in E. coli Rosetta2 (DE3) and purified with affinity and size-exclusion chromatography. Bacteria were handled as described in (1). The cells were disrupted by pulsed ultrasonic with micro-tip (Branson Sonifier Cell Disruptor B15, Branson Ultrasonics Corporation, USA) in a solution containing $100 \mathrm{mM}$ Tris-HCl, $\mathrm{pH}$ 7.4, $500 \mathrm{mM} \mathrm{NaCl}, 1 \mathrm{mM}$ phenylmethylsulfonyl fluoride (PMSF) and $1 \mathrm{mM}$ dithiothreitole (DTT). Cell debris was removed by centrifugation (50000 x g, $\left.4{ }^{\circ} \mathrm{C}, 30 \mathrm{~min}\right)$. The supernatant was applied onto a HisTrap column (GE Healthcare, GB) pre-equilibrated with $100 \mathrm{mM}$ Tris-HCl, pH 7.4, $500 \mathrm{mM} \mathrm{NaCl}, 1 \mathrm{mM}$ DTT. For the elution, $30 \%$ elution buffer (100 mM Tris-HCl, pH 7.4, $200 \mathrm{mM} \mathrm{NaCl,} 500 \mathrm{mM}$ imidazole, $1 \mathrm{mM}$ DTT) was used. Fractions containing the protein of interest were pooled and applied onto a size exclusion chromatography S200 gel filtration column (GE Healthcare, GB), which had been preequilibrated with $50 \mathrm{mM}$ Tris-HCl, $\mathrm{pH} 8.0,10 \mathrm{mM} \mathrm{MgCl}_{2}, 1 \mathrm{mM}$ DTT. Both chromatographies were operated with an ÄKTAprime plus system (GE Healthcare, GB) at $4^{\circ} \mathrm{C}$. The expression as well as the purity of the proteins was verified by SDS-PAGE and visualized with Coomassie Brilliant Blue staining. Protein aliquots were stored at $-80{ }^{\circ} \mathrm{C}$ without noticeable loss of activity. 
Preparation of ISC. ISC was synthesized by incubating $0.4 \mathrm{mg} / \mathrm{ml}$ His-ICS1 with either 1 or $0.1 \mathrm{mM}$ CA in $100 \mu \mathrm{L}$ of $50 \mathrm{mM}$ Tris- $\mathrm{HCl}$, pH 7.4 with $5 \mathrm{mM} \mathrm{MgCl} 2,10 \mathrm{mM} \mathrm{NaCl}$ and $1 \mathrm{mM}$ DTT for $10 \mathrm{~min}$ at $30{ }^{\circ} \mathrm{C}$ and shaking at $150 \mathrm{rpm}$. The protein was subsequently removed from the solution by ultrafiltration $\left(25^{\circ} \mathrm{C}, 16000 \mathrm{xg}\right)$ using a SpinX UF 500 concentrator (10,000 MWCO; Corning, USA). The product of the ICS1 reaction was monitored by UHPLCQ-TOF-MS analyses.

For the qualitative PBS3 activity assay, reactions were performed in $100 \mu \mathrm{L}$ of $50 \mathrm{mM}$ Tris$\mathrm{HCl}$, pH 7.8 with $5 \mathrm{mM} \mathrm{MgCl}$, $4 \mathrm{mM}$ glutamic acid, $5 \mathrm{mM}$ ATP, $0.6 \mathrm{mg} / \mathrm{mL}$ His-PBS3 and $6 \mu \mathrm{M}$ ISC or $50 \mu \mathrm{M}$ CA. The reaction was performed for $1 \mathrm{~h}$ at $30^{\circ} \mathrm{C}$, shaking at $150 \mathrm{rpm}$ and subsequently stopped by addition of $20 \mu \mathrm{L}$ acetonitrile. The products were monitored by UHPLC-Q-TOF-MS analyses.

Determination of the kinetic constants

To monitor the activity of PBS3 quantitatively, a spectrophotometric assay was employed. The formation of AMP by the PBS3 reaction was coupled to the reactions of pyruvate kinase, myokinase and lactate dehydrogenase (3). The assays were performed at $30{ }^{\circ} \mathrm{C}$ in $200 \mu \mathrm{L}$ of $50 \mathrm{mM}$ Tris- $\mathrm{HCl}$, pH 7.8 with $5 \mathrm{mM} \mathrm{MgCl}_{2}, 50 \mathrm{mM} \mathrm{KCl}, 0.5 \mathrm{mM}$ phosphoenolpyruvate, $4 \mathrm{mM}$ glutamic acid, $2 \mathrm{mM}$ ATP, 4 units of rabbit muscle pyruvate kinase (P1506, Sigma, Germany), 4 units of rabbit muscle myokinase (M3003, Sigma, Germany) and 4 units of rabbit muscle lactate dehydrogenase (L2500, Sigma, Germany) and $125 \mu \mathrm{M}$ NADH. The kinetic constants of PBS3 were determined for 4HBA (99\%, H20059, Aldrich, Germany) and ISC as acyl-substrates. Thereby, $0-1000 \mu \mathrm{M}$ of $4 \mathrm{HBA}$ and $50 \mu \mathrm{g} / \mathrm{mL}$ His-PBS3 respectively 0-35 $\mu \mathrm{M}$ ISC and $0.5 \mu \mathrm{g} / \mathrm{mL}$ His-PBS3 were used. The activity of His-PBS3 at lower protein concentration was stabilized with $0.13 \%$ (w/v) BSA (8076.2, Roth, Germany). The initial velocity was measured using a V-630 spectrophotometer (Jasco, Germany) by monitoring the absorbance change at $340 \mathrm{~nm}$ over $600 \mathrm{~s}$ at $30^{\circ} \mathrm{C}$.

Transient expression and subcellular localization

To determine the subcellular localization of PBS3, pET24-PBS3 was sub-cloned into pENTR vector utilizing the pENTR/D-TOPO Cloning Kit (Invitrogen, USA). The sequence confirmed constructs were used in an LR reaction with the binary destination vector pXCSGYFP (4) to generate $p X C S G$ 35S::PBS3::YFP. To determine potential co-localization, full length ICS1 was amplified from cDNA of Psm. infected Col0 leaves (1). Full length ICS1 was ultimately cloned into pXCSG-CFP (4) to generate pXCSG 35S::ICS1::CFP. For the construction of a plastidial PBS3, the first 47 amino acids of ICS1 were cloned upstream of PBS3. The full construct was transformed into the pXCSG-YFP vector to generate $p X C S G$ 35S::chloroPBS3::YFP. Additionally, a pUC18 derived pEntry vector containing a C-terminal eYFP coding sequence was utilized, to prepare a pEntry-35S::PBS3::YFP construct. Employing an LR reaction, this construct was integrated into the binary pCambia vector to yield pCambia-35S::PBS3::YFP. For transient expression into Arabidopsis leaves, we followed the protocol from (5) but used a higher Agrobacterium tumefaciens GV3101 concentration $\left(\mathrm{OD}_{600}=0.4\right)$ to enable SA quantification by UHPLC-Q-TOF-MS 24 hours after infiltration.

Confocal laser scanning microscopy was performed on transiently transformed A. thaliana efr leaves, 3 days after co-infiltration of Agrobacteria carrying the pXCSG 35S::ICS1::CFP and pCambia-35S::PBS3::YFP respectively plastidial pXCSG 35S::chloroPBS3::YFP constructs. The efr mutant background was used to achieve higher transformation efficiencies to allow fluorescence detection (6). Bacterial strains were co-infiltrated with a respective final $\mathrm{OD}_{600}$ of 0.3 together with the p19 suppressor. All images were taken with 20x objective (HC PL APO, CS2) of a TSC-SP8 FALCON microscope (Leica, Bensheim, Germany). ICS1-CFP was 
excited using a pulsed $440 \mathrm{~nm}$ diode laser, chloroPBS3-YFP was excited using $514 \mathrm{~nm}$ of a pulsed white light laser. Fluorescence emission was detected at 465-485 nm for CFP and 525$560 \mathrm{~nm}$ for YFP using Leica HyD SDM detectors, while chlorophyll autofluorescence was detected at 710-770 nm using a Leica HyD detector. Images were sequentially scanned. Merging was performed using Fiji software (7). 


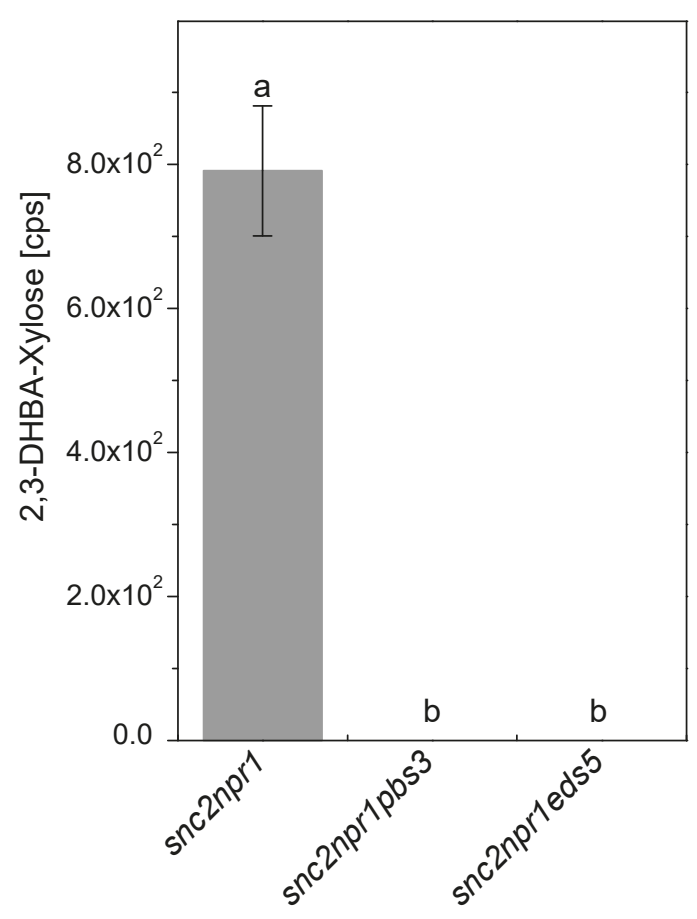

Fig. S1. Accumulation of 2,3-DHBA-Xyloside in snc2-1D npr1-1. The SA catabolite 2,3DHBA-Xyloside accumulates constitutively in snc2-1D npr 1-1, but not in snc2-1D npr11 pbs3-1 or snc2-1D npr1-1 eds5-3. Relative compound amounts (counts per second [cps]) were determined by LC-MS analysis of Arabidopsis thaliana leaf samples. Bars represent the mean \pm STD of three biological replicates. Statistical differences among replicates are labeled with different letters ( $\mathrm{P}<0.05$, one-way ANOVA and post hoc Tukey's Test; $\mathrm{n}=3$ ). 

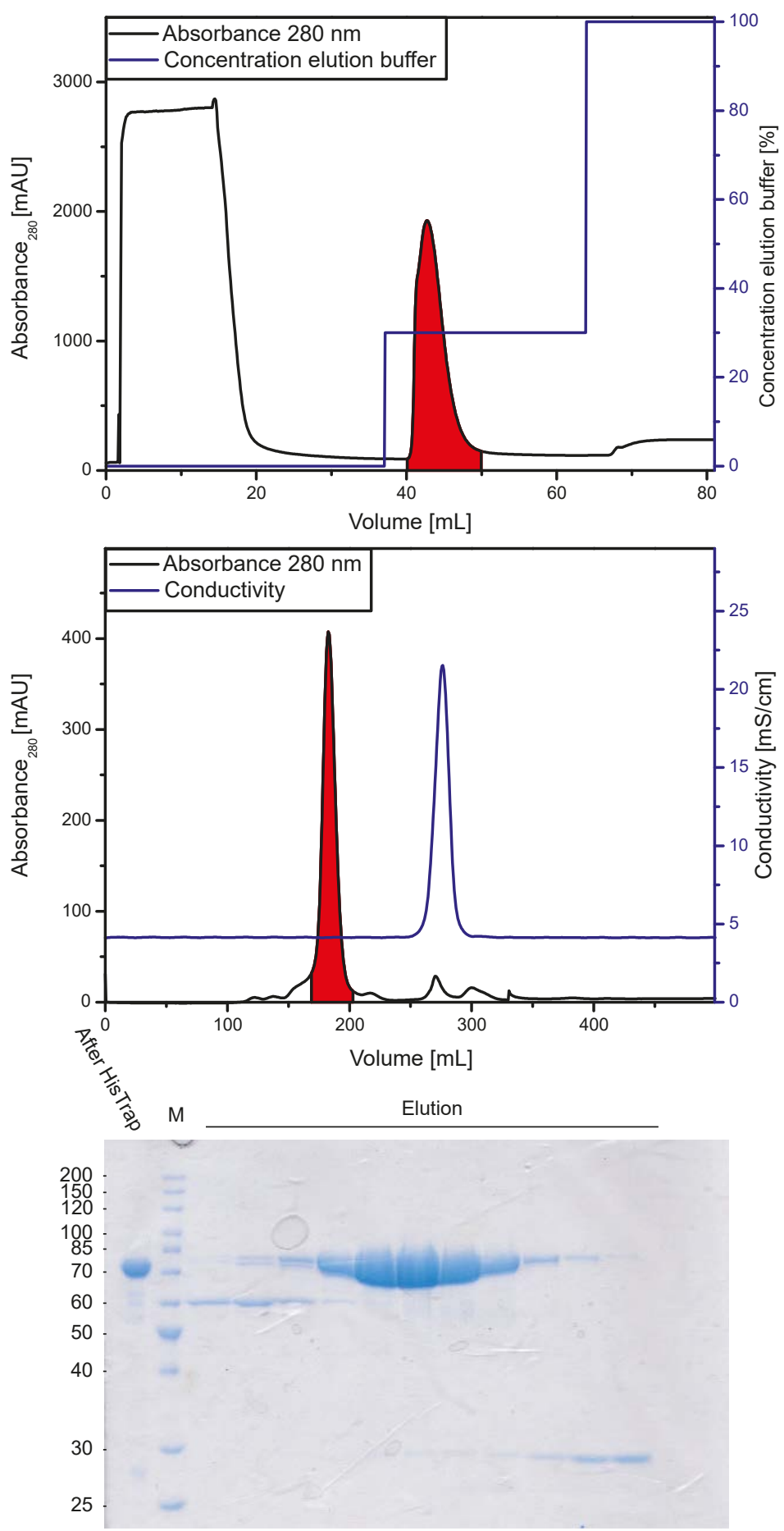

Fig. S2. Protein purification of His6-tagged AtPBS3 via affinity - and size exclusion chromatography. PBS3 was heterologously expressed in $E$. coli and subsequently purified via (A) affinity chromatography. Fractions corresponding to the area marked in red were collected and applied to (B) size exclusion chromatography. Fractions containing proteins were collected and examined by (C) SDS-PAGE to verify the purity. Pure protein containing fractions with the predicted molecular mass were pooled and concentrated by filter centrifugation. 
A

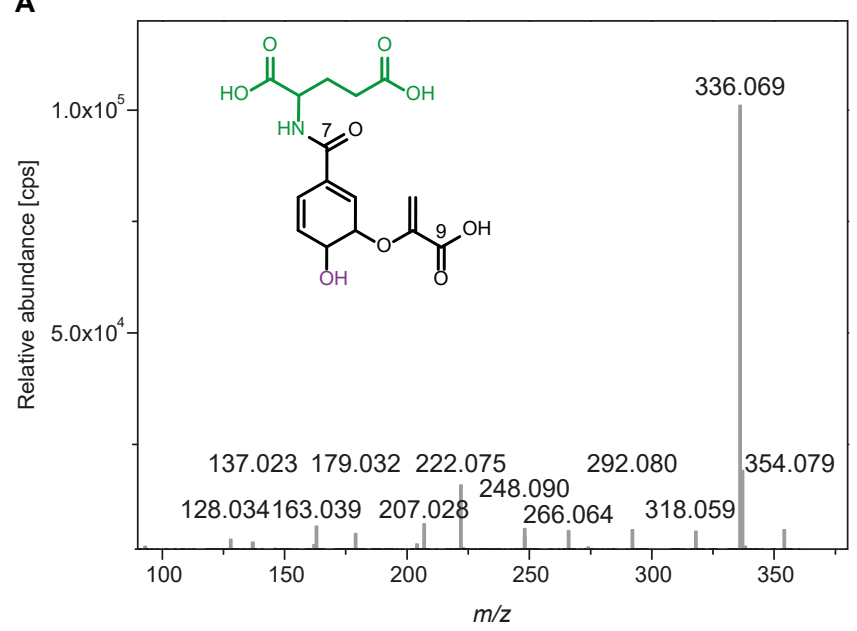

C

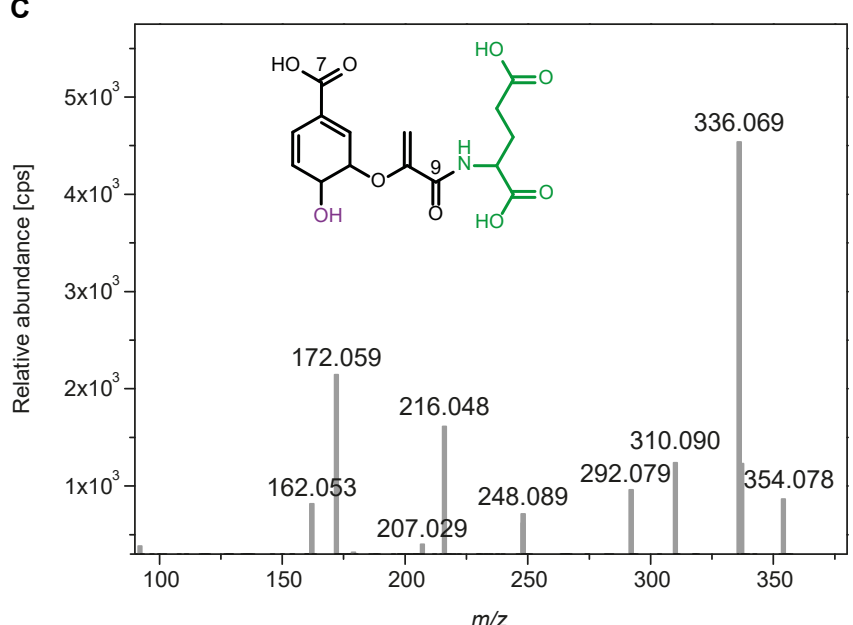

E

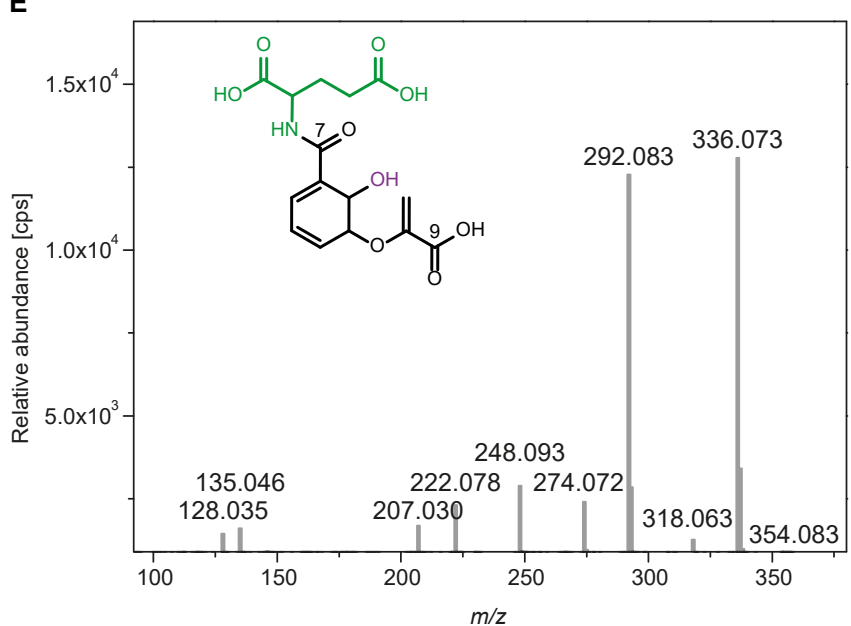

B

\begin{tabular}{|c|c|c|}
\hline $\begin{array}{l}\text { MS }^{2} \text { fragment of } \\
\text { CA-7-Glu }[\mathrm{m} / \mathrm{z}]\end{array}$ & $\begin{array}{c}\text { Elemental } \\
\text { composition }\end{array}$ & Identity \\
\hline 354.079 & $\mathrm{C}_{15} \mathrm{H}_{16} \mathrm{NO}_{9}$ & {$[\mathrm{M}-\mathrm{H}]^{-}$} \\
\hline 336.069 & $\mathrm{C}_{15} \mathrm{H}_{14} \mathrm{NO}_{8}$ & {$\left[\mathrm{M}-\mathrm{H}_{2} \mathrm{O}-\mathrm{H}\right]^{-}$} \\
\hline 318.059 & $\mathrm{C}_{15} \mathrm{H}_{13} \mathrm{NO}_{7}$ & {$\left[\mathrm{M}-2 \mathrm{H}_{2} \mathrm{O}\right]^{-}$} \\
\hline 292.080 & $\mathrm{C}_{14} \mathrm{H}_{14} \mathrm{NO}_{6}$ & {$\left[\mathrm{M}-\mathrm{H}_{2} \mathrm{O}-\mathrm{CO}_{2}-\mathrm{H}\right]^{-}$} \\
\hline 266.064 & $\mathrm{C}_{12} \mathrm{H}_{12} \mathrm{NO}_{6}$ & {$\left[\mathrm{M}-\mathrm{C}_{3} \mathrm{H}_{4} \mathrm{O}_{3}-\mathrm{H}\right]^{-}$} \\
\hline 248.090 & $\mathrm{C}_{13} \mathrm{H}_{14} \mathrm{NO}_{4}$ & {$\left[\mathrm{M}-\mathrm{H}_{2} \mathrm{O}-2 \mathrm{CO}_{2}-\mathrm{H}\right]^{-}$} \\
\hline 222.075 & $\mathrm{C}_{11} \mathrm{H}_{12} \mathrm{NO}_{4}$ & {$\left[\mathrm{M}-\mathrm{C}_{3} \mathrm{H}_{4} \mathrm{O}_{3}-\mathrm{CO}_{2}-\mathrm{H}\right]$} \\
\hline 207.028 & $\mathrm{C}_{10} \mathrm{H}_{7} \mathrm{O}_{5}$ & [M-Glu-H] \\
\hline 179.032 & $\mathrm{C}_{9} \mathrm{H}_{7} \mathrm{O}_{4}$ & [M-Glu-CO-H] ${ }^{-}$ \\
\hline 163.039 & $\mathrm{C}_{9} \mathrm{H}_{7} \mathrm{O}_{3}$ & {$\left[\mathrm{M}-\mathrm{Glu}-\mathrm{CO}_{2}-\mathrm{H}\right]^{-}$} \\
\hline 137.023 & $\mathrm{C}_{7} \mathrm{H}_{5} \mathrm{O}_{3}$ & {$\left[\mathrm{M}-\mathrm{Glu}-\mathrm{C}_{3} \mathrm{H}_{3} \mathrm{O}_{2}\right]^{-}$} \\
\hline 128.034 & $\mathrm{C}_{5} \mathrm{H}_{6} \mathrm{NO}_{3}$ & {$\left[\mathrm{Glu}-\mathrm{H}_{2} \mathrm{O}-\mathrm{H}\right]$} \\
\hline
\end{tabular}

D

\begin{tabular}{|c|c|c|}
\hline $\begin{array}{c}\mathrm{MS}^{2} \text { fragment of } \\
\text { CA-9-Glu }[\mathrm{m} / \mathrm{z}]\end{array}$ & $\begin{array}{c}\text { Elemental } \\
\text { composition }\end{array}$ & Identity \\
\hline 354.078 & $\mathrm{C}_{15} \mathrm{H}_{1} \mathrm{NO}_{9}$ & {$[\mathrm{M}-\mathrm{H}]$} \\
\hline 336.069 & $\mathrm{C}_{15} \mathrm{H}_{14} \mathrm{NO}_{8}$ & {$\left[\mathrm{M}-\mathrm{H}_{2} \mathrm{O}-\mathrm{H}\right]$} \\
\hline 310.090 & $\mathrm{C}_{14} \mathrm{H}_{16} \mathrm{NO}_{7}$ & {$\left[{\left.\mathrm{M}-\mathrm{CO}_{2}-\mathrm{H}\right]}^{-}\right.$} \\
\hline 292.079 & $\mathrm{C}_{14} \mathrm{H}_{14} \mathrm{NO}_{6}$ & {$\left[\mathrm{M}-\mathrm{H}_{2} \mathrm{O}-\mathrm{CO}_{2}-\mathrm{H}\right]$} \\
\hline 248.089 & $\mathrm{C}_{13} \mathrm{H}_{14} \mathrm{NO}_{4}$ & {$\left[\mathrm{M}-\mathrm{H}_{2} \mathrm{O}-2 \mathrm{CO}_{2}-\mathrm{H}\right]$} \\
\hline 207.029 & $\mathrm{C}_{10} \mathrm{H}_{7} \mathrm{O}_{5}$ & {$[\mathrm{M}-\mathrm{Glu}-\mathrm{H}]$} \\
\hline 216.048 & $\mathrm{C}_{8} \mathrm{H}_{10} \mathrm{NO}_{6}$ & {$[\mathrm{M}-\mathrm{SA}-\mathrm{H}]$} \\
\hline 172.059 & $\mathrm{C}_{7} \mathrm{H}_{10} \mathrm{NO}_{4}$ & {$\left[\mathrm{M}-\mathrm{SA}_{4}-\mathrm{CO}_{2}-\mathrm{H}\right]$} \\
\hline 162.053 & $\mathrm{C}_{9} \mathrm{H}_{8} \mathrm{NO}_{2}$ & {$\left[\mathrm{M}-\mathrm{CO}_{2}-\mathrm{H}_{2} \mathrm{O}-\mathrm{C}_{5} \mathrm{H}_{6} \mathrm{O}_{4}-\mathrm{H}\right]$} \\
\hline & \multicolumn{3}{|c}{} \\
\hline
\end{tabular}

\begin{tabular}{|c|c|c|}
\hline $\begin{array}{c}\text { MS fragment of } \\
\text { ISC-7-Glu }[\mathrm{m} / \mathrm{z}]\end{array}$ & $\begin{array}{c}\text { Elemental } \\
\text { composition }\end{array}$ & Identity \\
\hline 354.083 & $\mathrm{C}_{15} \mathrm{H}_{16} \mathrm{NO}_{9}$ & {$[\mathrm{M}-\mathrm{H}]$} \\
\hline 336.073 & $\mathrm{C}_{15} \mathrm{H}_{14} \mathrm{NO}_{8}$ & {$\left[\mathrm{M}-\mathrm{H}_{2} \mathrm{O}-\mathrm{H}\right]$} \\
\hline 318.063 & $\mathrm{C}_{15} \mathrm{H}_{13} \mathrm{NO}_{7}$ & {$\left[\mathrm{M}-2 \mathrm{H}_{2} \mathrm{O}\right]$} \\
\hline 292.083 & $\mathrm{C}_{14} \mathrm{H}_{14} \mathrm{NO}_{6}$ & {$\left[\mathrm{M}-\mathrm{H}_{2} \mathrm{O}-\mathrm{CO}_{2}-\mathrm{H}\right]$} \\
\hline 274.072 & $\mathrm{C}_{14} \mathrm{H}_{12} \mathrm{NO}_{5}$ & {$\left[\mathrm{M}-2 \mathrm{H}_{2} \mathrm{O}-\mathrm{CO}_{2}-\mathrm{H}\right]$} \\
\hline 248.093 & $\mathrm{C}_{13} \mathrm{H}_{14} \mathrm{NO}_{4}$ & {$\left[\mathrm{M}^{-} \mathrm{H}_{2} \mathrm{O}-2 \mathrm{CO}_{2}-\mathrm{H}\right]$} \\
\hline 222.078 & $\mathrm{C}_{11} \mathrm{H}_{12} \mathrm{NO}_{4}$ & {$\left[\mathrm{M}-\mathrm{C}_{3} \mathrm{H}_{4} \mathrm{O}_{3}-\mathrm{CO}_{2}-\mathrm{H}\right]^{-}$} \\
\hline 207.030 & $\mathrm{C}_{10} \mathrm{H}_{7} \mathrm{O}_{5}$ & {$[\mathrm{M}-\mathrm{Glu}-\mathrm{H}]^{-}$} \\
\hline 135.046 & $\mathrm{C}_{8} \mathrm{H}_{7} \mathrm{O}_{2}$ & {$\left[\mathrm{M}-\mathrm{Glu}-\mathrm{CO}-\mathrm{CO}_{2}-\mathrm{H}\right]$} \\
\hline 128.035 & $\mathrm{C}_{5} \mathrm{H}_{6} \mathrm{NO}_{3}$ & {$\left[\mathrm{Glu}-\mathrm{H}_{2} \mathrm{O}-\mathrm{H}\right]$} \\
\hline
\end{tabular}

Fig. S3. MS/MS fragmentations of PBS3 in vitro products CA-7-Glu, CA-9-Glu and ISC7-Glu. PBS3 activity assay was performed with Glu, ATP and chorismate (CA) (A, C) or isochorismate (ISC) (E) as acyl-substrate. MS/MS fragmentation pattern of (A) CA-7-Glu, (C) CA-9-Glu and (E) ISC-7-Glu with the corresponding annotation lists for the depicted fragments $(\mathbf{B}, \mathbf{D}, \mathbf{F})$. The identification is based on an accurate mass analysis. 

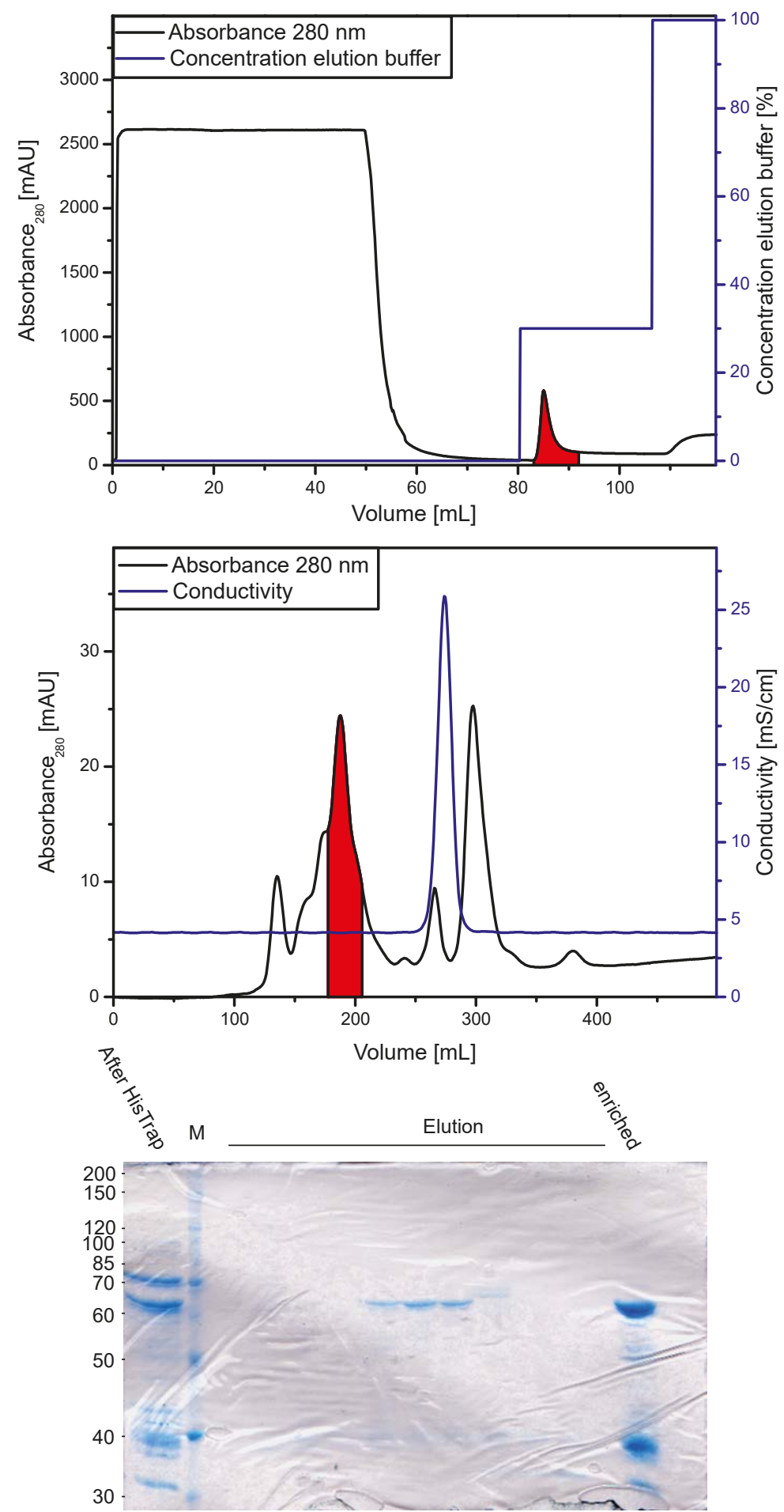

Fig. S4. Protein purification of His6-tagged AtICS1 via affinity - and size exclusion chromatography. ICS1 was heterologously expressed in $E$. coli and subsequently purified via (A) affinity chromatography. Fractions corresponding to the area marked in red were collected and applied to (B) size exclusion chromatography. Fractions containing proteins were collected and examined by (C) SDS-PAGE to verify the purity. Pure protein containing fractions with the predicted molecular mass were pooled and concentrated by filter centrifugation. 
A

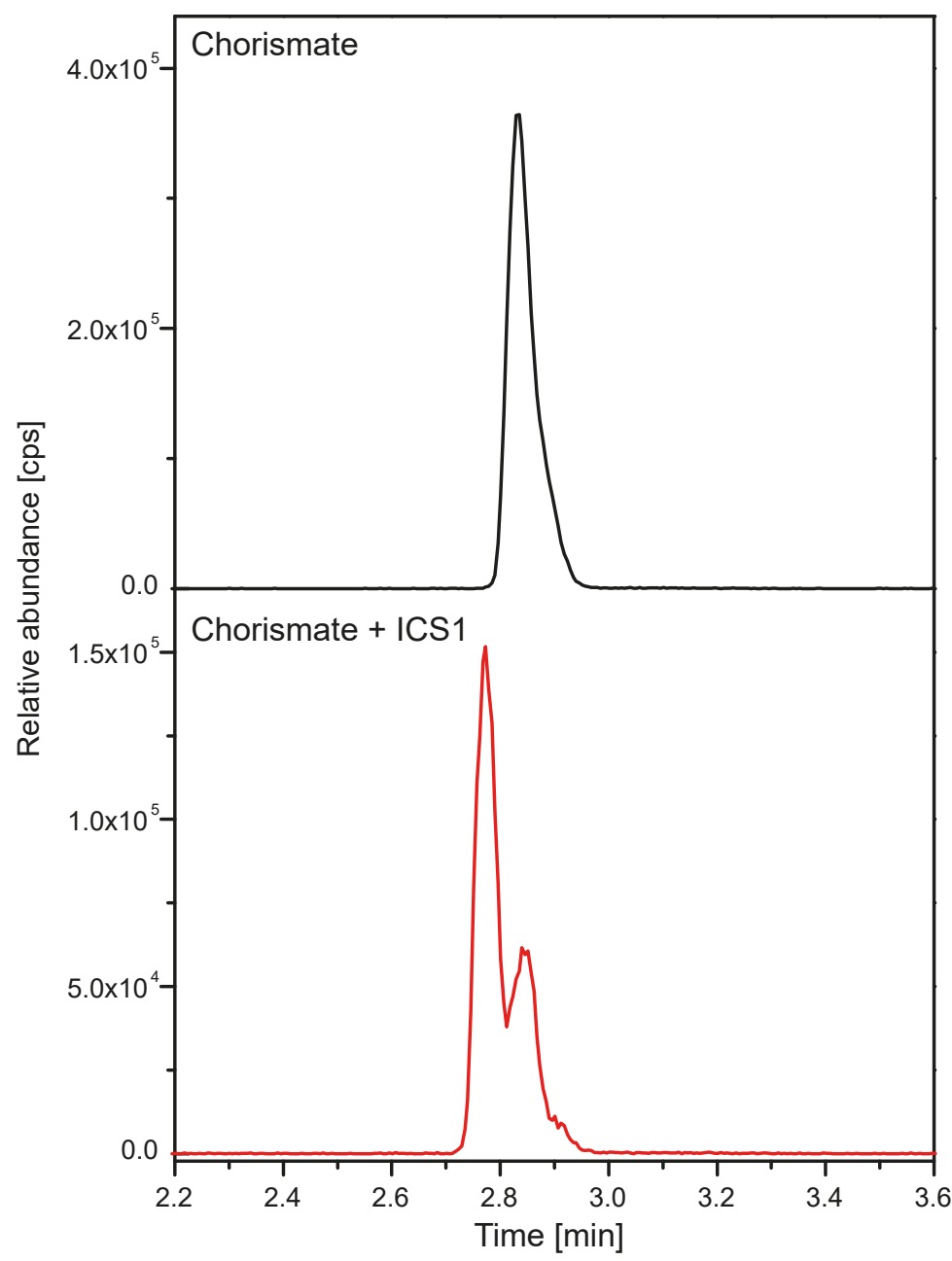

B

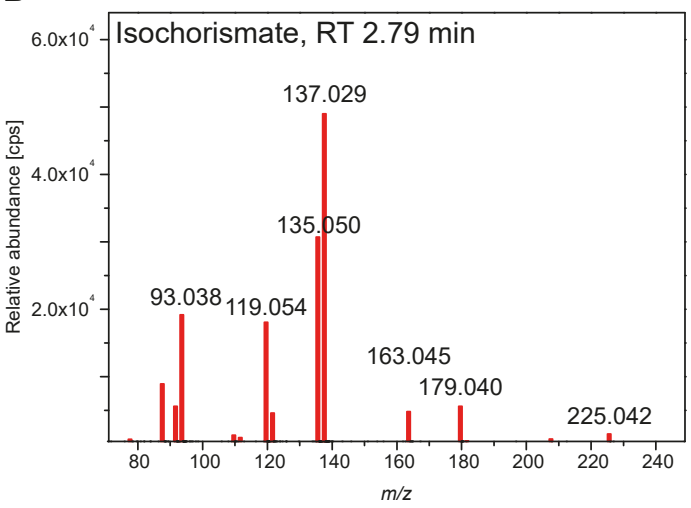

C

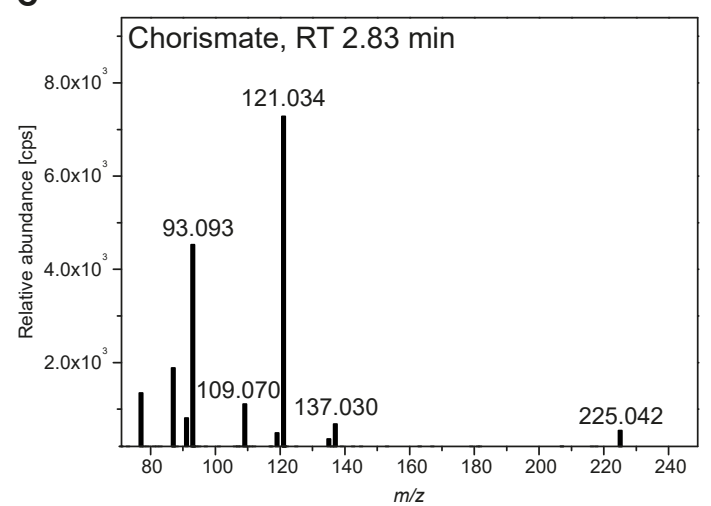

Fig. S5 Activity assay of ICS1 yields isochorismate. LC-MS analysis was performed on activity assays of purified ICS1 with chorismate (CA). (A) The extracted ion chromatogram for CA/ISC $(\mathrm{m} / \mathrm{z} 225.042)$ is shown. Upper panel without -, lower panel with enzyme. (B) $\mathrm{MS} / \mathrm{MS}$ fragmentation for ISC from the activity assay at $2.79 \mathrm{~min}$. (C) MS/MS fragmentation for CA from the activity assay without enzyme at $2.83 \mathrm{~min}$. ISC and CA can be distinguished based on retention time shift and differences in their fragmentation pattern. RT: retention time. 
A

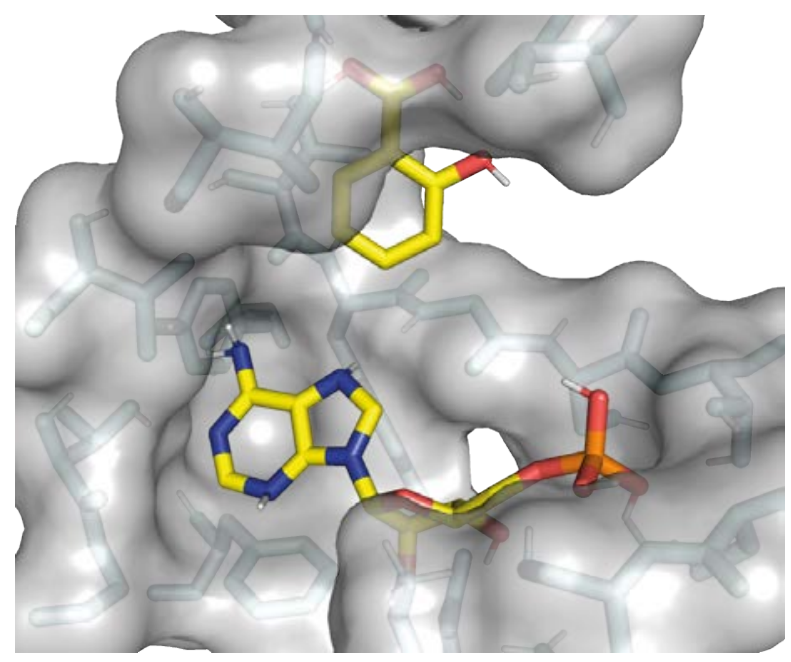

C

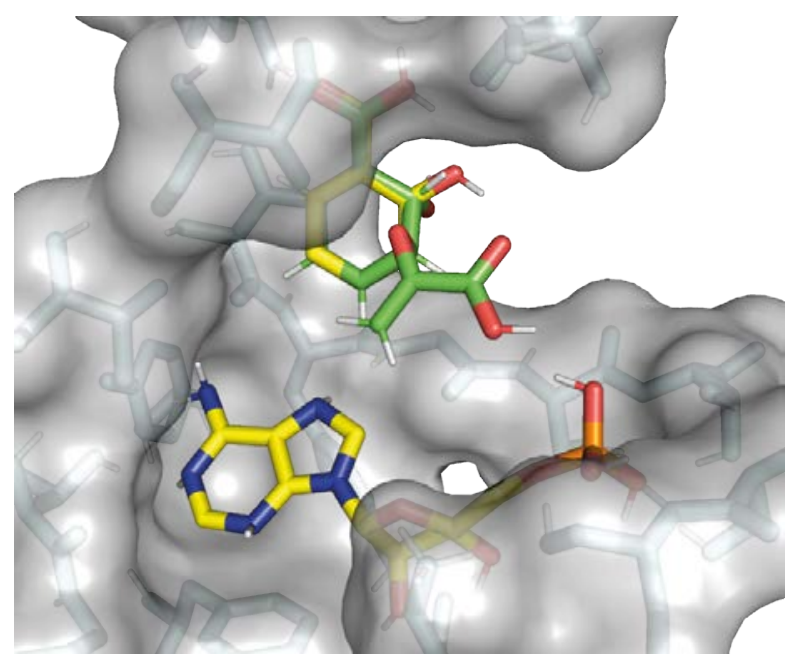

B

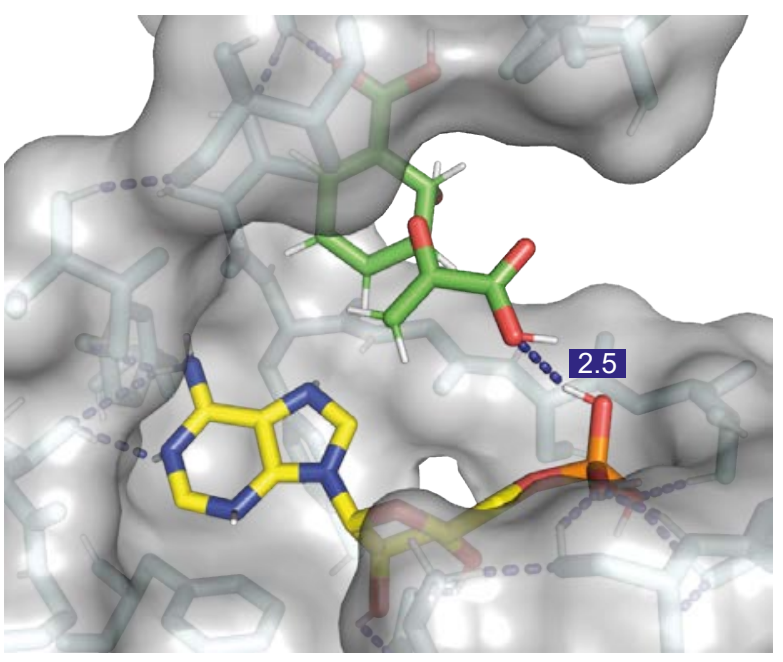

D

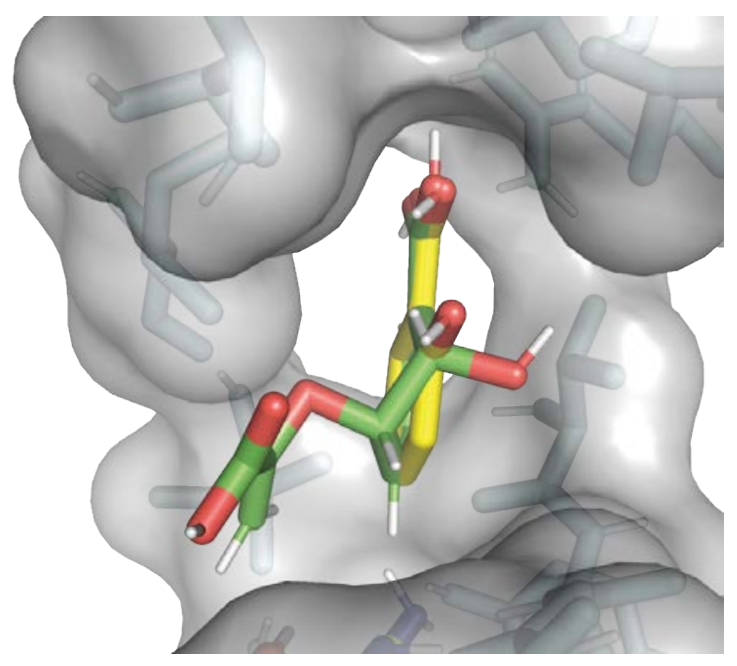

Fig. S6. Isochorismate fits into the active site of PBS3. For substrate modeling, the crystal structure of PBS3-AMP-SA (PDB ID 4eql) was used (8). Residues in a radius of $4 \AA$ are shown as gray surface to visualize the binding pocket of AMP and the acyl substrate. C-atoms of AMP and SA are shown in yellow, for isochorismate (ISC) in green. (A) The large distance between the carboxyl group of SA and the phosphate group of AMP was previously proposed to be the reason why SA is a poor substrate for PBS3 (8). (B) The hydroxy acrylic group of ISC points towards the phosphate group of AMP. The presented model is based on the SA position in the crystal structure and shows proximity between the carboxyl - and the phosphate group $(\sim 2.5 \AA)$. (C, D) Overlay of SA (yellow) and ISC (green) in the binding pocket of PBS3. Although ISC is slightly larger than SA, there appears to be no negative interference with any surrounding residues. This figure was generated with PyMOL (version 2.2.0, Schrödinger, USA). 
A

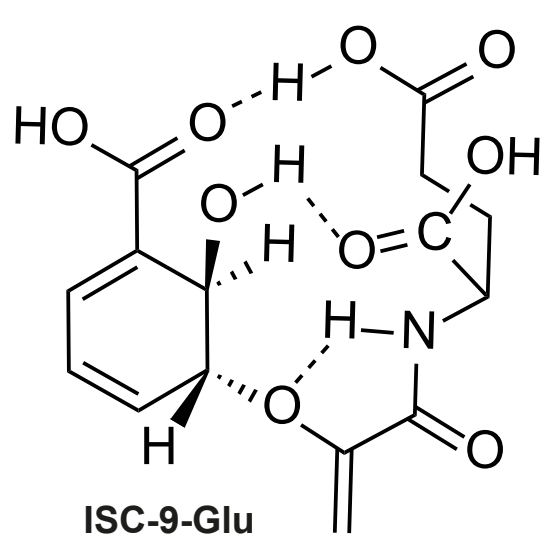

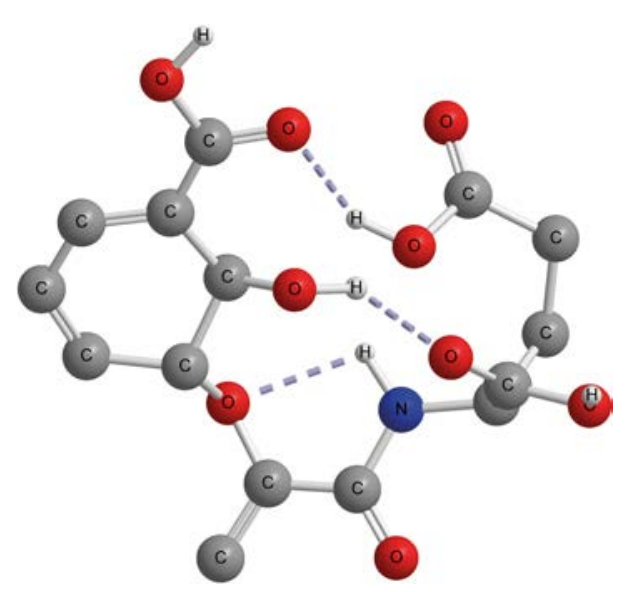

B

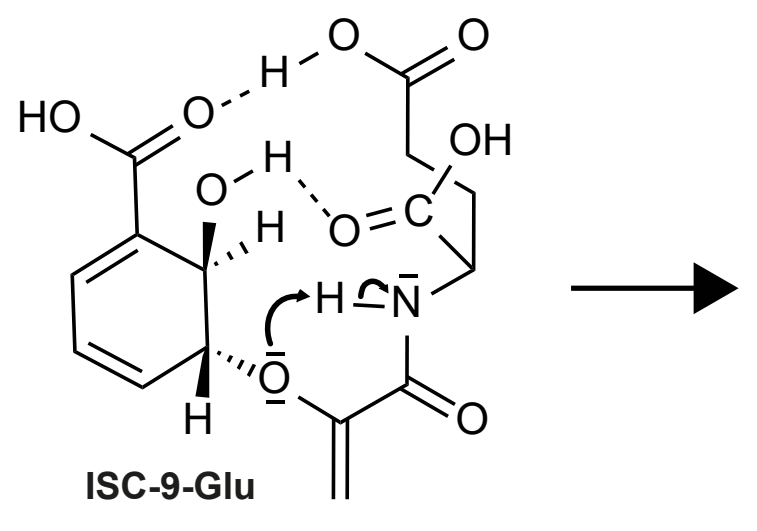

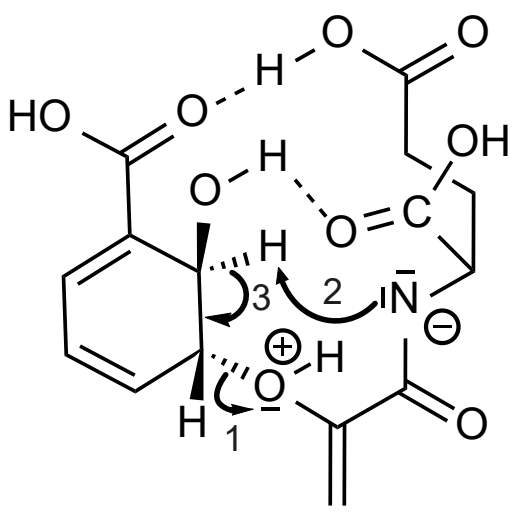<smiles>O=C(O)c1ccccc1O</smiles><smiles>C=C(O)C(=O)NC(CCC)(CCC(=O)O)C(=O)O</smiles>

Fig. S7. Molecular model of ISC-9-Glu and its decay to SA and 2HNG. (A) Molecular modeling was used to generate a 3D model of ISC-9-Glu. This figure was generated with Chem3D (version 17.1 PerkinElmer, USA) (B) The formation of the intramolecular hydrogen bonds facilitates the hydrogen transfer from the amide hydrogen onto the ether oxygen. Subsequently, the non-enzymatic decay of ISC-9-Glu probably follows an E1 elimination mechanism. In the first part of the reaction, a heterolytic C-O cleavage occurs, which is enhanced by the previous protonation of the linker-oxygen. In the second part, we propose a base initiated aromatization of the ring structure. The final products of this elimination are SA and $2 \mathrm{HNG}$. 


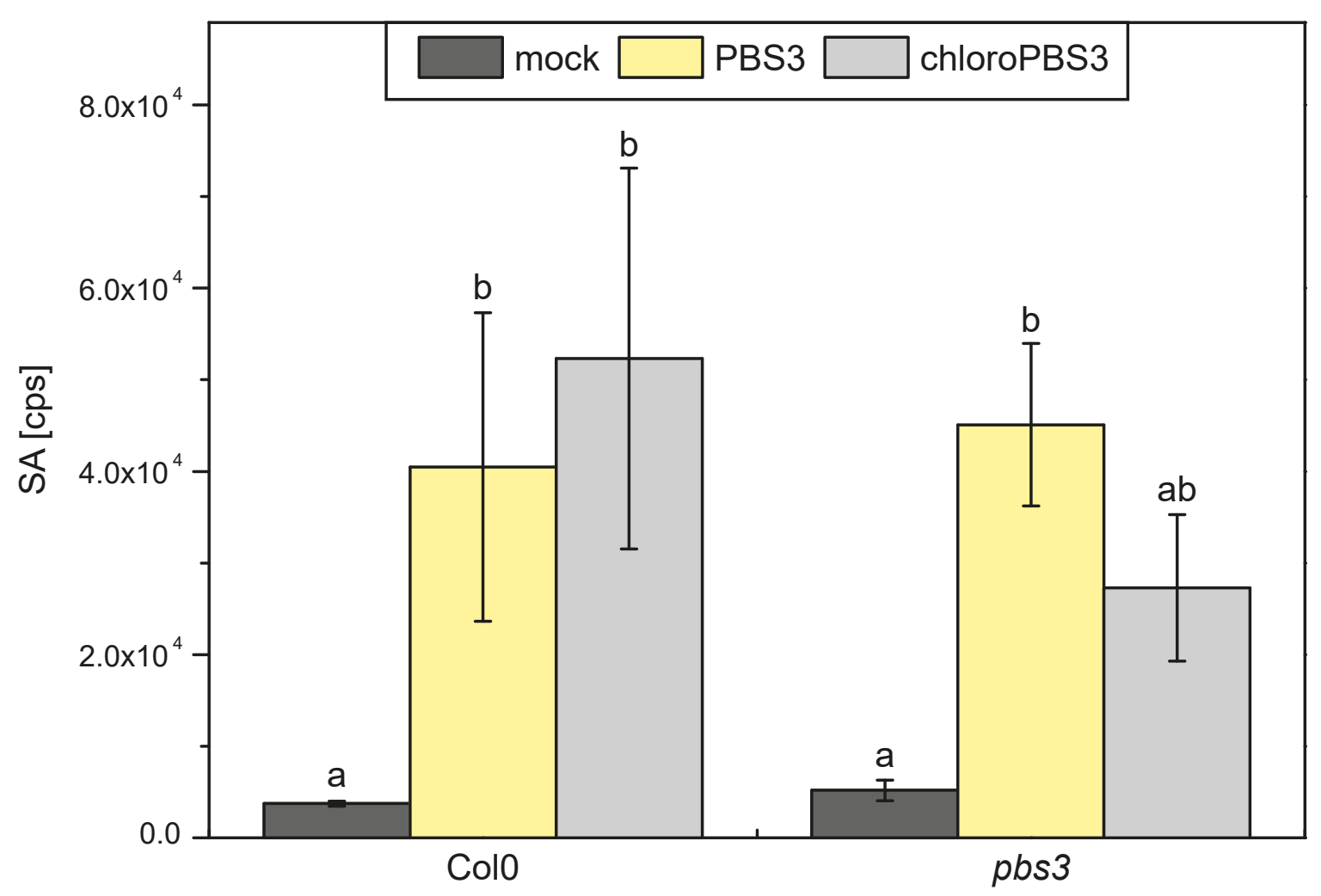

Fig. S8. Agrobacterium induced SA production in Col0 and pbs3-1. The transient expression of PBS3 or chloroPBS3 in Arabidopsis Co10 or pbs3-1 leaves restores the Agrobacterium tumefaciens induced SA accumulation. $24 \mathrm{~h}$ after infiltration, leaves were collected and metabolites were extracted as described for Metabolite fingerprint analysis. Infiltration medium was used as mock treatment. The SA content was analyzed by UHPLC-Q-TOF-MS. Bars represent the mean \pm STD of three biological replicates. Statistical differences among replicates are labeled with different letters $(\mathrm{P}<0.05$, one-way ANOVA and post hoc Tukey's Test; $\mathrm{n}=3$ ). 
Table S1. Fragmentation data for investigated metabolites.

\begin{tabular}{|l|c|l|c|c|c|}
\hline & Type of ion & Elemental composition & Exact mass [Da] & MS/MS fragmentation & CE [eV] \\
\hline CA-7-Glu & {$[\mathrm{M}-\mathrm{H}]-$} & $\mathrm{C} 15 \mathrm{H} 17 \mathrm{NO} 9$ & 355.090 & $336.072,222.077,163.04$ \\
\hline CA-9-Glu & {$[\mathrm{M}-\mathrm{H}]-$} & $\mathrm{C} 15 \mathrm{H} 17 \mathrm{NO} 9$ & 355.090 & $336.072,216.051,172.061$ \\
\hline ISC-7-Glu & {$[\mathrm{M}-\mathrm{H}]-$} & $\mathrm{C} 15 \mathrm{H} 17 \mathrm{NO} 9$ & 355.090 & $292.082 .274 .072,135.045$ & 8 \\
\hline ISC-9-Glu & {$[\mathrm{M}-\mathrm{H}]-$} & $\mathrm{C} 15 \mathrm{H} 17 \mathrm{NO}$ & 355.090 & $292.082,216.051,172.061,137.024$ \\
\hline SA & {$[\mathrm{M}-\mathrm{H}]-$} & $\mathrm{C} 7 \mathrm{H} 6 \mathrm{O} 3$ & 138.032 & 93.034 & 10 \\
\hline 2HNG & {$[\mathrm{M}-\mathrm{H}]-$} & $\mathrm{C} 8 \mathrm{H} 11 \mathrm{NO}$ & 217.059 & $172.061,136.040,86.024$ \\
\hline CA & {$[\mathrm{M}-\mathrm{H}]-$} & $\mathrm{C} 10 \mathrm{H} 10 \mathrm{O}$ & 226.048 & $137.026,121.029,93.034$ \\
\hline ISC & {$[\mathrm{M}-\mathrm{H}]-$} & $\mathrm{C} 10 \mathrm{H} 10 \mathrm{O}$ & 226.048 & $137.026,135.045,119.050,93.034$ \\
\hline
\end{tabular}


Table S2. Primers used in this study.

\begin{tabular}{|l|l|l|}
\hline Primer & 5'-3' sequence & Purpose \\
\hline ICS1-D47_EcoRI_fwd & ACGGAATTCATGAATGGTTGTGATGGAGA & cloning \\
\hline ICS1-D47_HindllI_rev & ACGAAGCTTTCAATTAATCGCCTGTAGAGA & cloning \\
\hline ICS1_W_Tag_Sall_fwd & ACGGTCGACATGGCTTCACTTCAATTTCTTCT & cloning \\
\hline ICS1-TAG-Sall_rev & ACGGTCGACGAAATCTCCATCACAACCATT & cloning \\
\hline ICS1_TOPO_fwd & CACCATGGCTTCACTTCAATTTCTTC & cloning \\
\hline ICS1_TOPO_rev & ATTAATCGCCTGTAGAGATGTTG & cloning \\
\hline PBS3_BamHIfwd & ACGGGATCCATGAAGCCAATCTTCGATATCAACG & cloning \\
\hline PBS3_HindIII_rev & ACGAAGCTTAATACTGAAGAATTTGGTACCACAC & cloning \\
\hline PBS3_TOPO_fwd & CACCATGAAGCCAATCTTCGATATC & cloning \\
\hline PBS3_TOPO_rev & AATACTGAAGAATTGGCTACCAC & cloning \\
\hline
\end{tabular}




\section{References}

1. P. Ding et al., Characterization of a pipecolic acid biosynthesis pathway required for systemic acquired resistance. Plant Cell 28, 2603-2615 (2016).

2. A. Kaever et al., MarVis-Pathway: integrative and exploratory pathway analysis of non-targeted metabolomics data. Metabolomics 11, 764-777 (2015).

3. Q. Chen, C. S. Westfall, L. M. Hicks, S. Wang, J. M. Jez, Kinetic basis for the conjugation of auxin by a GH3 family indole-acetic acid-amido synthetase. J. Biol. Chem. 285, 29780-29786 (2010).

4. B. J. Feys et al., Arabidopsis SENESCENCE-ASSOCIATED GENE101 stabilizes and signals within an ENHANCED DISEASE SUSCEPTIBILITY1 complex in plant innate immunity. Plant Cell 17, 2601-2613 (2005).

5. T. Rosas-Díaz et al., Arabidopsis NahG Plants as a Suitable and Efficient System for Transient Expression using Agrobacterium tumefaciens. Mol. Plant 10, 353-356 (2017).

6. C. Zipfel et al., Perception of the bacterial PAMP EF-Tu by the receptor EFR restricts Agrobacterium-mediated transformation. Cell 125, 749-760 (2006).

7. J. Schindelin et al., Fiji: an open-source platform for biological-image analysis. Nature Methods 9, 676 (2012).

8. C. S. Westfall et al., Structural basis for prereceptor modulation of plant hormones by GH3 proteins. Science 336, 1708-1711 (2012). 


\section{Article III. Is Enhanced Disease Susceptibility 5 the Achilles' Heel of Plant Immunity?}

The article is ready for submission. The supplementary materials are attached to the main part.

\section{Author contribution:}

Dmitrij Rekhter planned and performed the metabolite fingerprint analysis as well as the subsequent metabolite identification by high resolution UHPLC-Q-TOF-MS/MS analysis. He performed the UV-C as well as chemical treatment. He analyzed, processed, displayed, interpreted and discussed the results, and wrote the first draft of the manuscript. 


\section{Is Enhanced Disease Susceptibility 5 the Achilles' Heel of Plant Immunity?}

Dmitrij Rekhter ${ }^{1}$, Lennart Mohnike ${ }^{1}$, Kirstin Feussner ${ }^{1,2}$, Krzysztof Zienkiewicz ${ }^{1,2}$, Yuelin Zhang $^{3, *}$, Ivo Feussner ${ }^{1,2,4, *}$

${ }^{1}$ University of Goettingen, Albrecht-von-Haller-Institute for Plant Sciences, Department of Plant Biochemistry, D-37077 Goettingen, Germany.

${ }^{2}$ University of Goettingen, Goettingen Center for Molecular Biosciences (GZMB), Service Unit for Metabolomics and Lipidomics, D-37077 Goettingen, Germany.

${ }^{3}$ University of British Columbia, Department of Botany, Vancouver, BC V6T 1Z4, Canada.

${ }^{4}$ University of Goettingen, Goettingen Center for Molecular Biosciences (GZMB), Department of Plant Biochemistry, D-37077 Goettingen, Germany.

*Correspondence to Ivo Feussner: ifeussn@uni-goettingen.de or Yuelin Zhang: yuelin.zhang@ubc.ca.

Pipecolic acid (Pip) is essential for the establishment of systemic acquired resistance (SAR) in plants. This plastid derived non-proteinogenic amino acid is further processed in the cytosol to its active form $\mathbf{N}$-hydroxy pipecolic acid (NHP) and $\mathbf{N}$-hydroxy pipecolic acid glycoside (NHP-OGIc). Here we provide strong evidence that the MATE-like exporter Enhanced Disease Susceptibility 5 (EDS5) exports Pip out of the plastid into the cytosol. This finding raises the question why no bacterial effector, which would target EDS5 and thereby explore this weak point of plant immunity, was found so far. 
Upon pathogen challenge, plants produce a number of metabolites, which are critical for the activation of the innate immune system ${ }^{1}$. At the site of infection as well as in systemic tissues the accumulation of salicylic acid (SA) and pipecolic acid (Pip) is a key event for the successful defense against biotrophic pathogens ${ }^{2}$. The first step in the SA biosynthesis, the conversion of chorismic acid (CA) to isochorismic acid (ISC) by Isochorismate Synthase 1 (ICS1), occurs in plastids ${ }^{3}$. The precise mechanism for the subsequent formation of SA is not understood yet. However, the plastid localized MATE like transport Enhanced Disease Susceptibility 5 (EDS5) was shown to be essential for the biosynthesis of SA $\mathrm{A}^{4}$. An intact Gretchen Hagen enzyme 3.12, also known as avrPphB Susceptible 3 (PBS3), is required for pathogen induced SA biosynthesis ${ }^{5}$. Although its exact biochemical role is not known yet, it seems likely to act upstream of SA. A knockout in any of these three genes leads to a drastic reduction of pathogen induced SA production and consequently to a higher pathogen susceptibility. In case of Pip, both biosynthetic enzymes, AGD2-like Defense Response Protein 1 (ALD1) and SAR-Deficient 4 (SARD4), are located in plastids as well ${ }^{6,7}$. For a successful activation of Pip signaling, a functional Flavin-dependent Monooxygenase 1 (FMO1) is required. Recently, two independent groups showed that FMO1 is responsible for the $\mathrm{N}$-oxygenation of Pip to yield $\mathrm{N}$-hydroxy pipecolic acid (NHP) ${ }^{8,9}$. This newly discovered compound was proposed to be a critical regulator of systemic acquired resistance (SAR) ${ }^{8}$. Although, the exact localization of FMO1 is not determined yet, studies of other FMOs strongly suggest a localization on the cytoplasmic surface of the endoplasmic reticulum ${ }^{10}$. Both, SA and NHP, can be further glycosylated to SA- $\beta$-glucoside (SAG) and SA-glucoseester (SGE) ${ }^{11}$ respectively to $N$-hydroxy pipecolic acid glycoside (NHP-OGIC) ${ }^{9}$. Glycosylation of plant hormones are typically facilitated by UDPglycosyltransferases (UGTs), of which the vast majority was allocated to the cytosol ${ }^{12}$. This strongly suggests that there must be a transporter from the site of Pip biosynthesis to the location where it is further processed. Not only pathogenic infection, but also abiotic stresses like ozone or UV-C treatment can induce the biosynthesis of SA and $\mathrm{SAG}^{13}$. In order to have a fast and reproducible test system for Pip synthesis, we tested the possibility to induce the accumulation of Pip by UV-C stress.

Indeed, Pip accumulates over time in Arabidopsis thaliana leaves in a similar course of time (Fig. 1a), as it was described for infection with Pseudomonas syringae before ${ }^{8}$. Beside Pip, we could also 
measure the accumulation of NHP and NHP-OGIc (MS/MS fragmentation is depicted in Supplementary Fig. 1$)^{9}$, what suggests that also the Pip oxidation and glycosylation occur after UV-C treatment. In order to exclude that the observed synthesis of Pip and NHP-OGIC is activated by UV-C stress induced SA accumulation, we examined Pip and NHP-OGlc content in the SA deficient mutants eds 5 and pbs3 with and without UV-C treatment. As expected, SA and SAG accumulate only in wild type, but not in the mutant lines 24 hours after the treatment with UV-C (Fig. 1b and c). Strikingly, Pip and NHP-OGIc accumulate only in wild type and pbs3, but not in the eds5 plants (Fig. $1 \mathrm{~d}$ and e). From the results in the pbs3 mutant we deduce that Pip biosynthesis and processing do not depend on the accumulation of SA in UV-C treated plants, comparable to the situation in pathogen treated plants ${ }^{8}$. However, the absence of Pip and NHP-OGlc in the eds5 raised the question, if EDS5 is responsible not only for the export of SA but also for Pip. To address the question if Pip or NHP is exported from the plastid, we investigated the reaction of $f m o 1$ to UV-C stress. As it was shown previously in pathogen assays ${ }^{8}, f m o 1$ also accumulates large amounts of Pip after UV-C treatment but is impaired in the hydroxylation of Pip into NHP (Supplementary Fig. 2). If FMO1 is located in plastids, fmo1 knockout plants would not be able to accumulate Pip upon UV-C treatment as observed in eds5 plants. As the accumulation of Pip in eds5 and fmo1 differs, we conclude that both enzymes are involved in Pip biosynthesis and EDS5 acts upstream from FMO1. We assume that the metabolic flux prevents a harmful accumulation of signaling compounds in plastids ${ }^{14}$. It is conceivable that either Pip inhibits its own biosynthesis via a feedback loop or feeds into the lysine degradation pathway towards the Krebs cycle ${ }^{15}$. Interestingly, we observed an accumulation of the SA precursor ISC in the pbs3 mutant plants upon UV-C treatment (Supplementary Fig. 3). This might indicate that PBS3 regulate SA biosynthesis by modifying the homeostasis of ISC. As PBS3 was recently shown to be located in the cytosol ${ }^{16}$, it is conceivable that EDS5 is exporting ISC instead of SA, which is then modified in the cytosol by PBS3.

In order to exclude subsidiary effects of the UV-C treatment, we investigated the reaction of eds5 and pbs3 plants to SA treatment (Fig. 2a). As expected, SA induces the biosynthesis of Pip and NHP-OGIc in wild type plants ${ }^{17}$. In the pbs3 mutant, the biosynthesis of SA is impaired but SA treatment can still induce Pip accumulation. For eds 5 plants however, it was not possible to induce the production of Pip 
with SA - as with UV-C treatment. These results strongly support our hypothesis that EDS5 is the plastidial exporter of Pip. When we use Pip irrigation to induce in planta Pip biosynthesis, we detect a significant accumulation of the downstream product NHP-OGlc in wild type plants (Fig. 2b). In eds5 and the Pip biosynthesis mutant sard4, this accumulation is reduced by more than $60 \%$. Therefore, we conclude that in the mutants only the external Pip was processed into NHP-OGlc, whereas de novo Pip biosynthesis could not be restored. The ability of eds 5 plants to convert exogenous Pip into NHP-OGIc confirms our hypothesis that FMO1 is active outside of plastids and thus, its activity is not affected by a mutation of EDS5.

Taking together, we show that UV-C treatment is sufficient to induce the production of Pip and its metabolites. This process is independent from SA signaling and can thus be used to study the SA independent branch of plant immunity. Moreover, we identified here a previously unknown connection between Pip and EDS5, a gene that, so far, was only recognized for its involvement in SA biosynthesis ${ }^{13}$. Instead, EDS5 is also responsible for the export of Pip from plastids into the cytosol, where it is further processed. EDS5 appears hereby as the central hub in the biosynthesis of both major signaling compounds, SA and NHP (Fig. 2c). If it is ISC and not SA that is exported from the plastid by EDS5, the similarity between NHP and SA biosynthesis becomes obvious. The spatial separation of biosynthesis and location of action adds an additional layer of regulation. Therefore, it is conceivable that both biosynthetic pathways utilize the same plastidial exporter. Keeping this in mind, it is astonishing that no bacterial effector, which would target EDS5 and thereby explore this weak point of plant immunity, was found so far. 
a

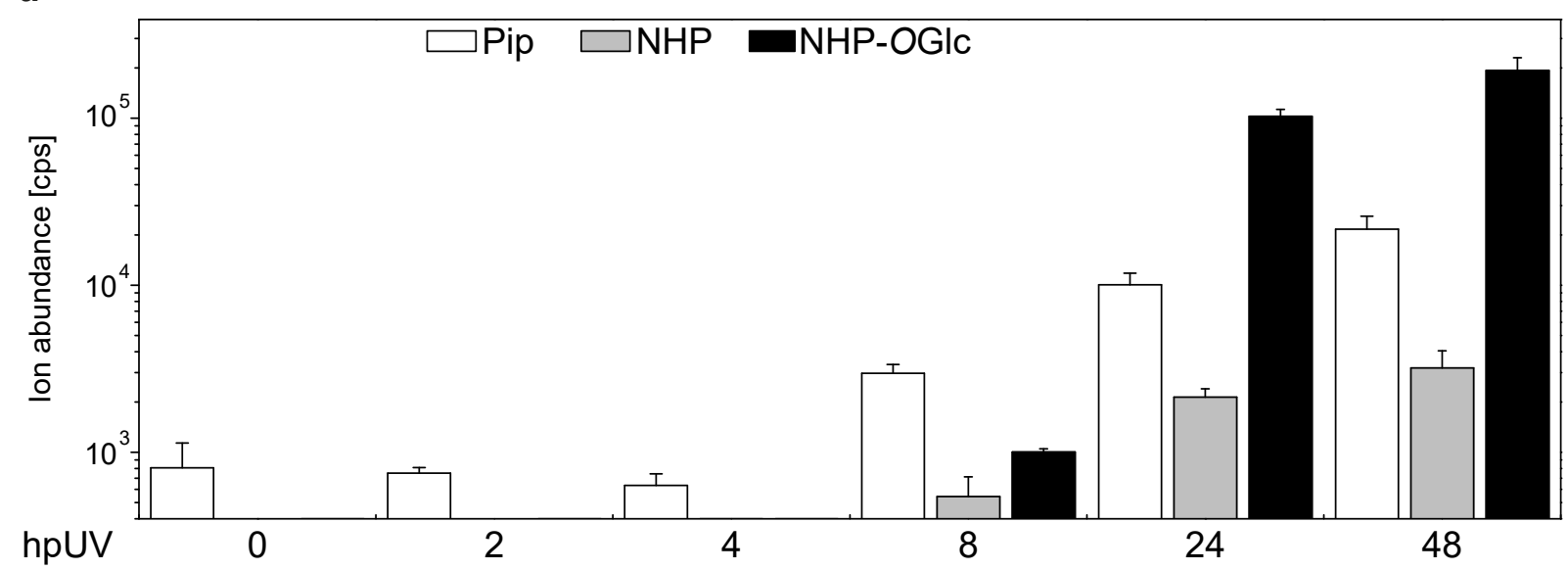

b

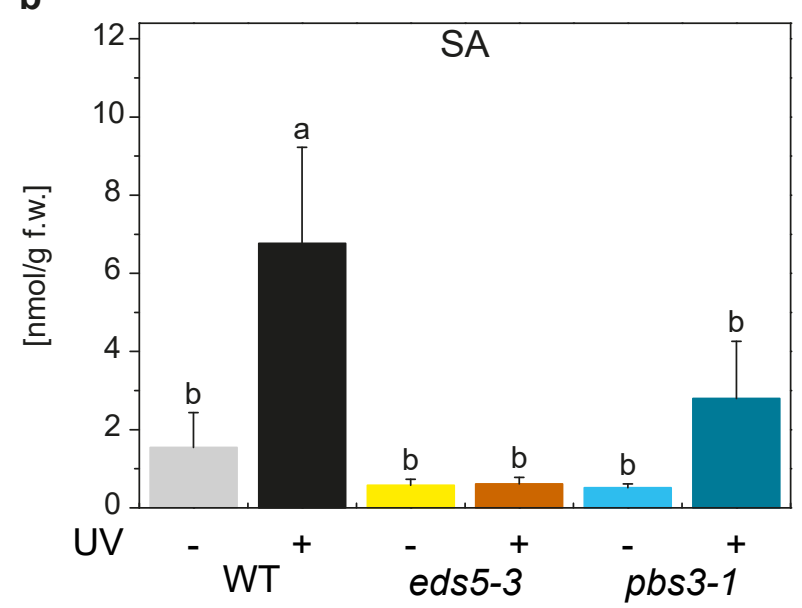

d

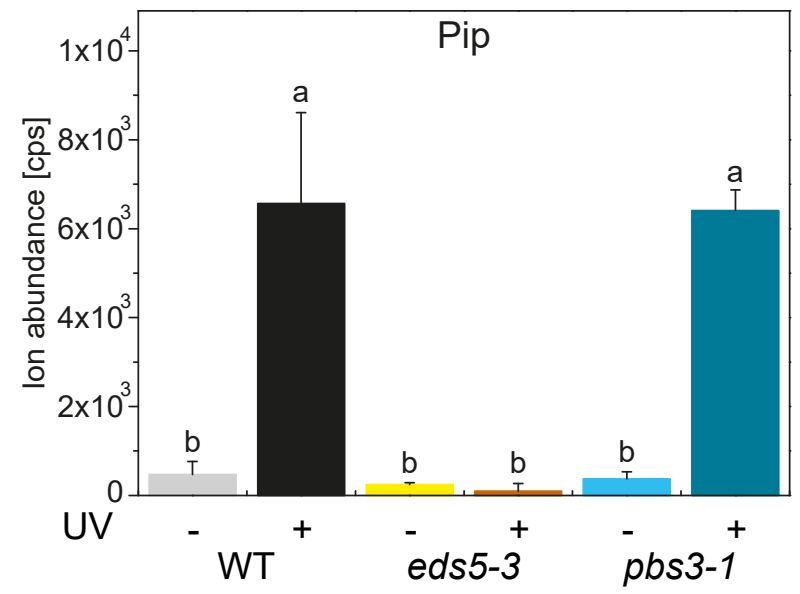

C

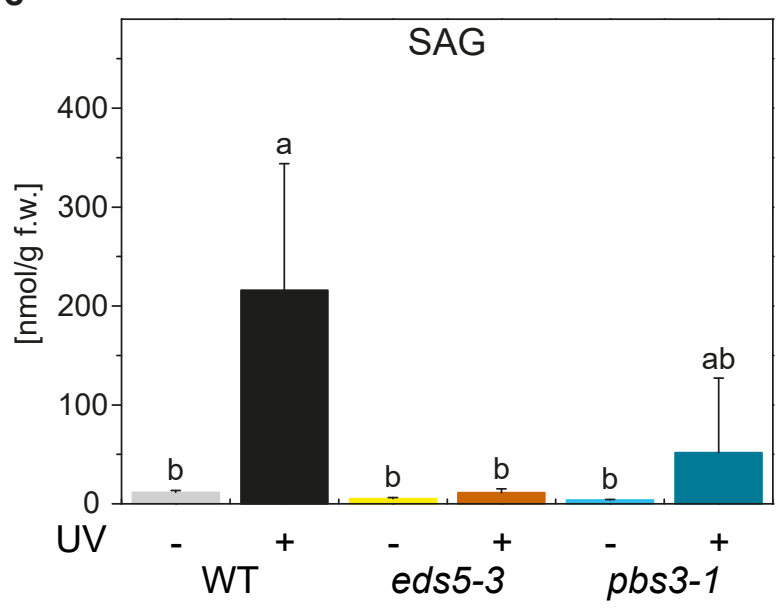

e

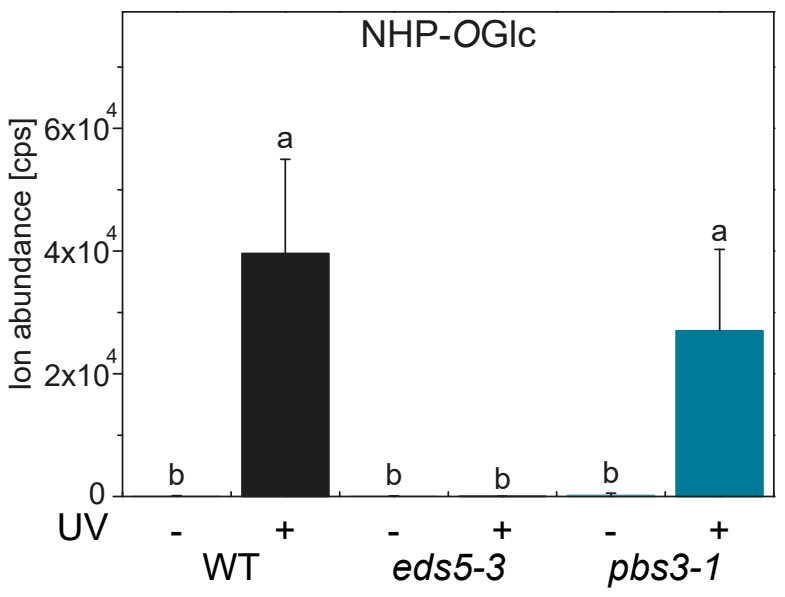

Figure 1 UV-C treatment of Arabidopsis thaliana leads to an accumulation of the signaling compounds SA and Pip and their corresponding glycosides. a, Levels of Pip (white bars) and its downstream products NHP (grey bars) and NHP-OGlc (black bars; counts per second [cps]) in Arabidopsis wild type leaves (Col-0) at different time points after UV-C light treatment. Levels of $\mathbf{b}$-c, SA and its glycoside SAG (nmol g-1 leaf fresh weight [f.w.]) and d-e, Pip and its glycoside NHP-OGlc (counts per second [cps]) in leaves of wild type (Col-0), respectively eds5-3 and pbs3-1 mutant plants 
24 hours after UV-C treatment in comparison to untreated plants. Bars represent the mean \pm STD of three biological replicates. Statistical differences among replicates are labeled with different letters (P $<0.05$, one-way ANOVA and post hoc Tukey's Test; $n=3$ ). The experiments were repeated twice with similar results. 
a

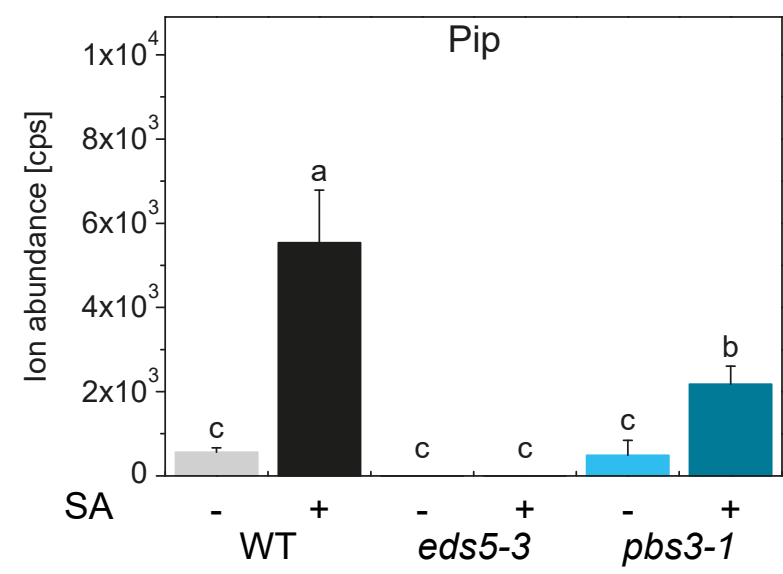

b

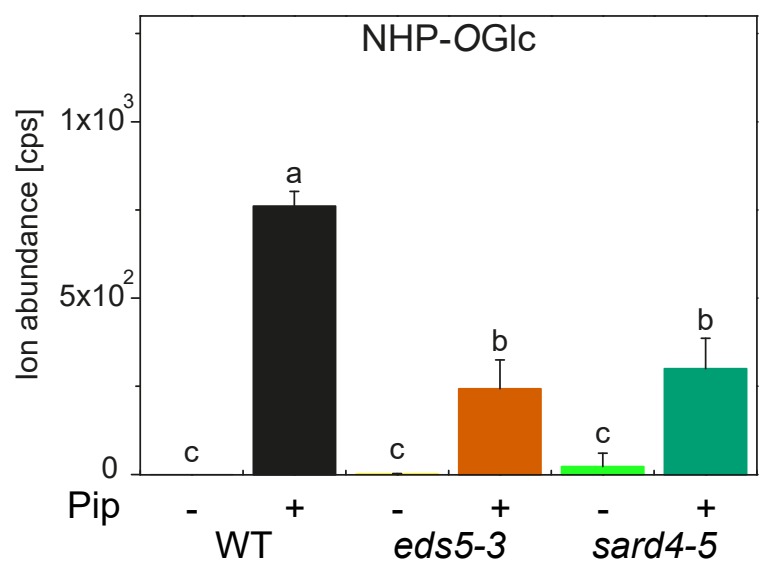

C

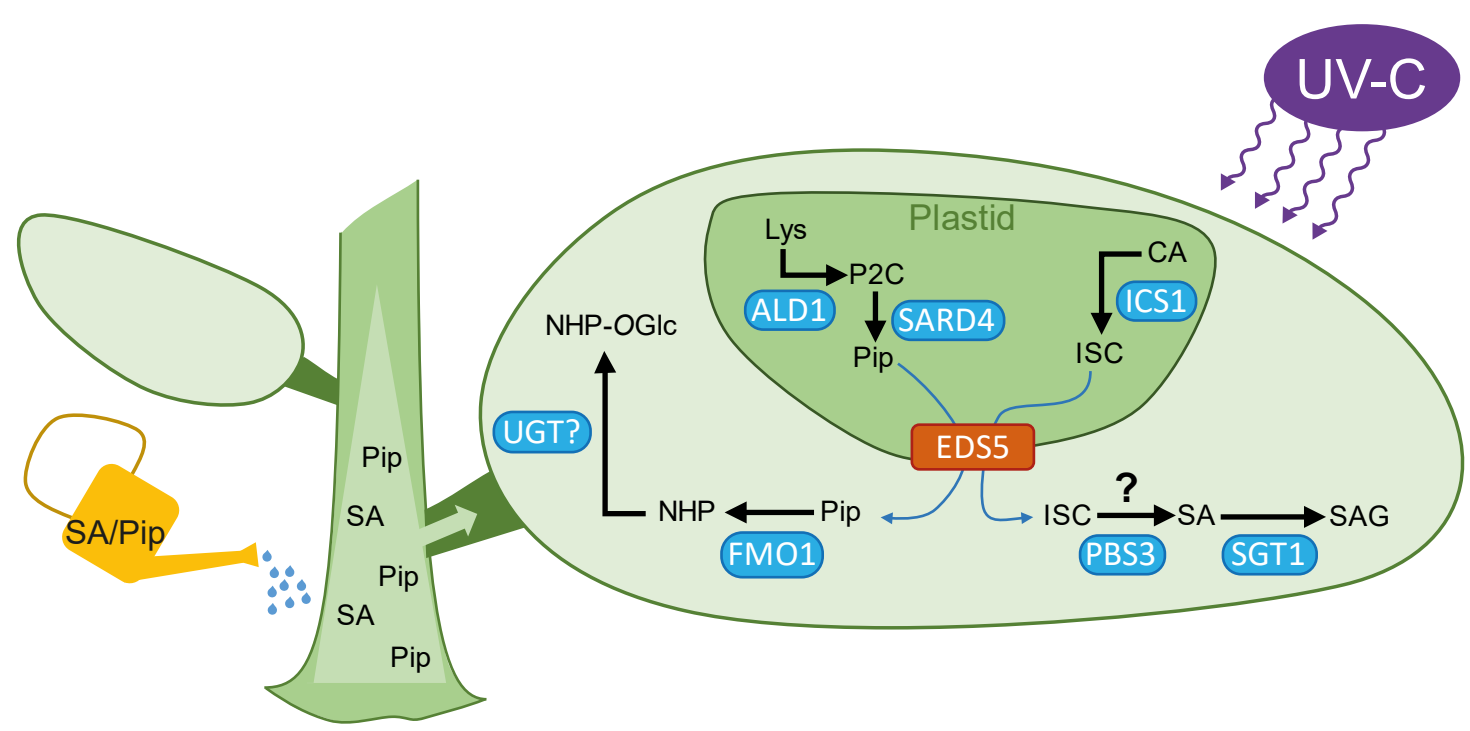

Figure 2 Root application of SA respectively Pip induces Pip biosynthesis and processing in leafs. a, Levels of Pip (counts per second [cps]) in leaves of wild type plants (Col-0), respectively in leafs of eds53 and pbs3-1 24 hours after soil drenching with water (-) or SA (+). b, Levels of the Pip downstream product NHP-OGlc (counts per second [cps]) in leafs of wild type plants (Col-0), respectively in leafs of eds5-3 and sard4-5 24 hours after soil drenching with water (-) or Pip (+). Bars represent the mean \pm STD of three biological replicates. Statistical differences among replicates are labeled with different letters ( $P<0.05$, one-way ANOVA and post hoc Tukey's Test; $n=3$ ). The experiment was repeated twice with similar results. c, A working model depicting EDS5 as the central hub in the biosynthesis of the signaling compounds SA and NHP. The induction of these production pathways was facilitated here by UV-C light treatment or soil drench with SA respectively Pip. 
a

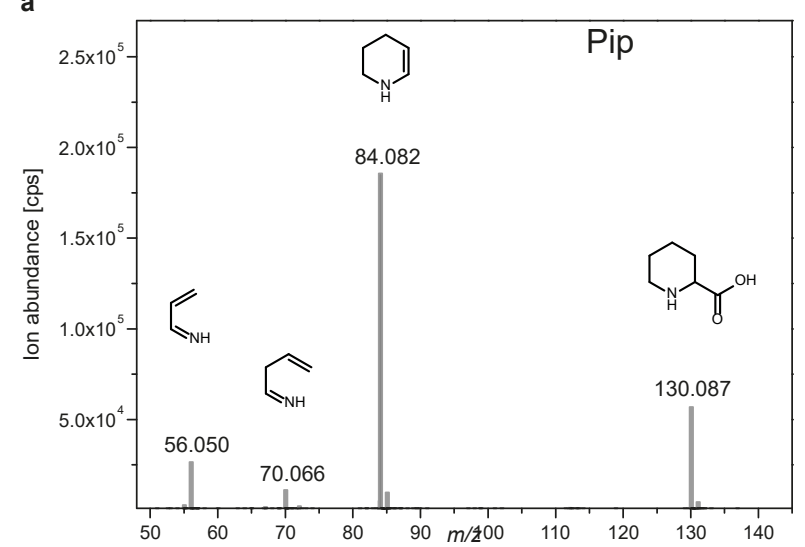

c

\begin{tabular}{|c|c|c|}
\hline $\begin{array}{c}\text { MS' } \text { fragment of } \\
\text { Pip [m/z] }\end{array}$ & $\begin{array}{c}\text { Elemental } \\
\text { composition }\end{array}$ & Identity \\
\hline 130.087 & $\mathrm{C}_{6} \mathrm{H}_{12} \mathrm{NO}_{2}$ & {$[\mathrm{M}+\mathrm{H}]^{+}$} \\
\hline 84.082 & $\mathrm{C}_{5} \mathrm{H}_{1} \mathrm{~N}$ & {$[\mathrm{M}-\mathrm{COOH}]^{+}$} \\
\hline 70.066 & $\mathrm{C}_{4} \mathrm{H}_{8} \mathrm{~N}$ & {$\left[\mathrm{M}-\mathrm{COOH}-\mathrm{CH}_{2}\right]^{+}$} \\
\hline 56.050 & $\mathrm{C}_{3} \mathrm{H}_{6} \mathrm{~N}$ & {$\left[\mathrm{M}-\mathrm{COOH}-2 \mathrm{CH}_{2}\right]^{+}$} \\
\hline
\end{tabular}

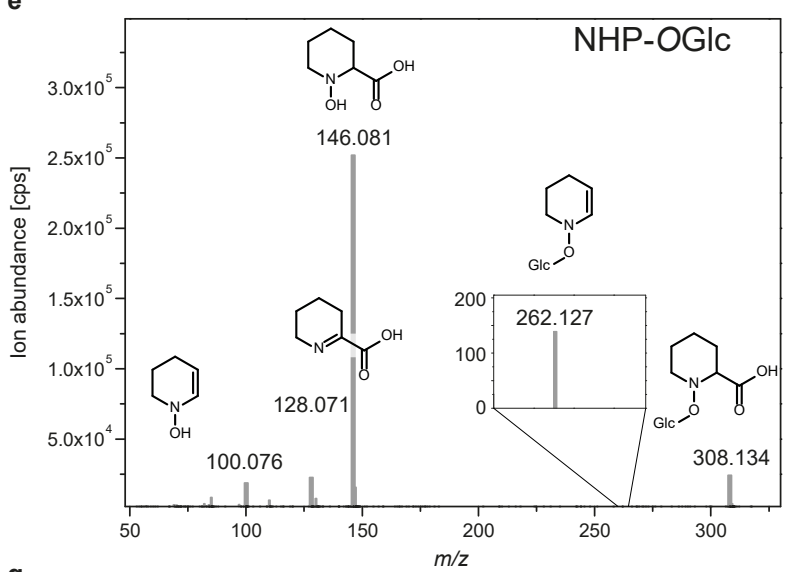

g

\begin{tabular}{|c|c|c|}
\hline $\begin{array}{c}\mathbf{M S}^{2} \text { fragment of } \\
\text { NHP-OGIc }[\mathbf{m} / \mathbf{z}]\end{array}$ & $\begin{array}{c}\text { Elemental } \\
\text { composition }\end{array}$ & Identity \\
\hline 308.134 & $\mathrm{C}_{12} \mathrm{H}_{22} \mathrm{NO}_{8}$ & {$[\mathrm{M}+\mathrm{H}]^{+}$} \\
\hline 262.127 & $\mathrm{C}_{11} \mathrm{H}_{20} \mathrm{NO}_{6}$ & {$[\mathrm{M}-\mathrm{COOH}]^{+}$} \\
\hline 146.081 & $\mathrm{C}_{6} \mathrm{H}_{12} \mathrm{NO}_{3}$ & {$\left[\mathrm{M}-\mathrm{Glc}+\mathrm{H}_{2} \mathrm{O}+\mathrm{H}^{+}\right.$} \\
\hline 128.071 & $\mathrm{C}_{6} \mathrm{H}_{10} \mathrm{NO}_{2}$ & {$[\mathrm{M}-\mathrm{Glc}+\mathrm{H}]^{+}$} \\
\hline 100.076 & $\mathrm{C}_{5} \mathrm{H}_{10} \mathrm{NO}$ & {$\left[\mathrm{M}-\mathrm{Glc}-\mathrm{COOH}+\mathrm{H}_{2} \mathrm{O}\right]^{+}$} \\
\hline
\end{tabular}

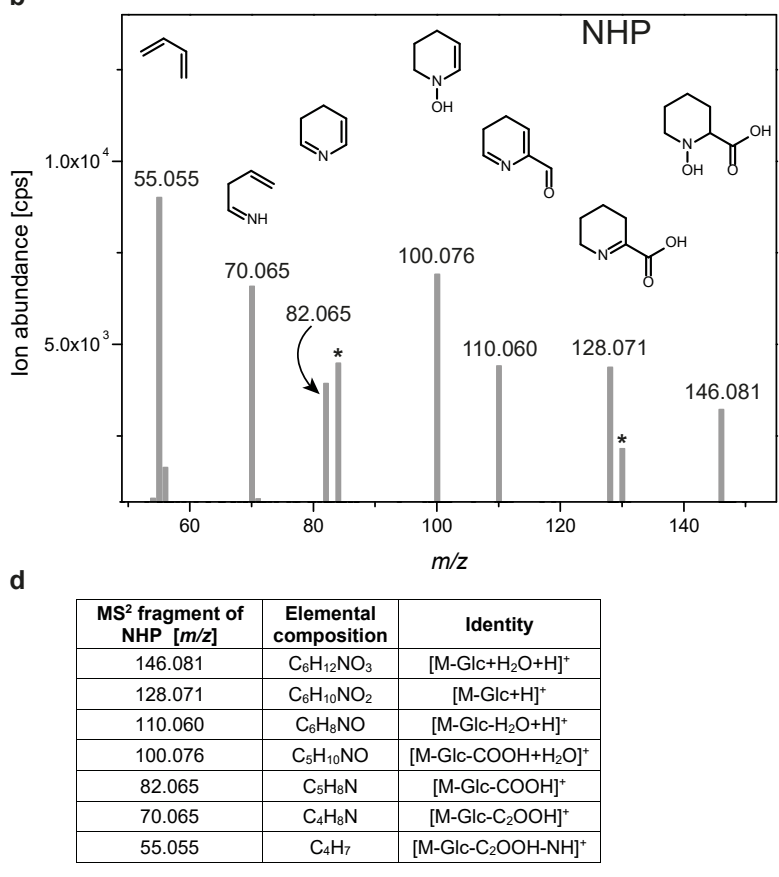

f

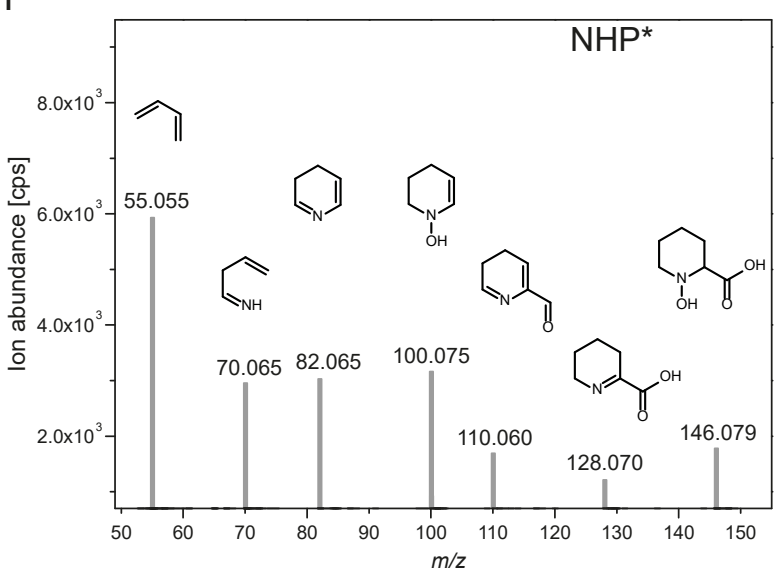

\begin{tabular}{|c|c|c|}
\hline $\begin{array}{c}\text { Pseudo MS }^{3} \\
\text { fragment of NHP- } \\
\text { OGlc }[\mathrm{m} / \mathrm{z}]\end{array}$ & $\begin{array}{l}\text { Elemental } \\
\text { composition }\end{array}$ & Identity \\
\hline 146.081 & $\mathrm{C}_{6} \mathrm{H}_{12} \mathrm{NO}_{3}$ & {$\left[\mathrm{M}-\mathrm{Glc}+\mathrm{H}_{2} \mathrm{O}+\mathrm{H}\right]^{+}$} \\
\hline 128.071 & $\mathrm{C}_{6} \mathrm{H}_{10} \mathrm{NO}_{2}$ & {$[\mathrm{M}-\mathrm{GlC}+\mathrm{H}]^{+}$} \\
\hline 110.060 & $\mathrm{C}_{6} \mathrm{H}_{8} \mathrm{NO}$ & {$\left[\mathrm{M}-\mathrm{Glc}-\mathrm{H}_{2} \mathrm{O}+\mathrm{H}\right]^{+}$} \\
\hline 100.076 & $\mathrm{C}_{5} \mathrm{H}_{10} \mathrm{NO}$ & {$\left[\mathrm{M}-\mathrm{Glc}-\mathrm{COOH}+\mathrm{H}_{2} \mathrm{O}\right]^{+}$} \\
\hline 82.065 & $\mathrm{C}_{5} \mathrm{H}_{8} \mathrm{~N}$ & {$[\mathrm{M}-\mathrm{Glc}-\mathrm{COOH}]^{+}$} \\
\hline 70.065 & $\mathrm{C}_{4} \mathrm{H}_{8} \mathrm{~N}$ & {$\left[\mathrm{M}-\mathrm{Glc}-\mathrm{C}_{2} \mathrm{OOH}\right]^{+}$} \\
\hline 55.055 & $\mathrm{C}_{4} \mathrm{H}_{7}$ & {$\left[\mathrm{M}-\mathrm{Glc}_{-} \mathrm{C}_{2} \mathrm{OOH}-\mathrm{NH}\right]^{+}$} \\
\hline
\end{tabular}

Supplementary Figure 1 Unambiguous identification of Pip, NHP and NHP-OGlc by high resolution

MS/MS fragmentation. Upon UV-C treatment, wild type plants produce substantial amounts of pipecolic acid (Pip), N-hydroxy pipecolic acid (NHP) and N-hydroxy pipecolic acid glycoside (NHP-OGlc). The same samples were investigate here as in Figure 1. MS/MS fragmentation spectra of $\mathbf{a}, \mathrm{Pip}$ and $\mathbf{b}$, NHP were verified by fragmentations of authentical standards. The fragments marked with a $*$ in the NHP fragmentation corresponds to sample background from insource fragment masses of glutamic 
acid, which elutes at a similar retention time. In e, the MS/MS fragmentation pattern of NHP-OGIc is depicted. NHP-OGlc fragmentizes already during the ionization process. In order to verify the identity of NHP-OGlc, the insource aglycon ion was selected for a subsequent MS/MS fragmentation. The pseudo $\mathrm{MS}^{3}$ fragmentation spectrum depicted in $\mathbf{f}$ unequivocally identifies the aglycon as NHP. The fragment annotation $(\mathbf{c}, \mathbf{d}, \mathbf{g}, \mathbf{h})$ is based on accurate mass analysis. 

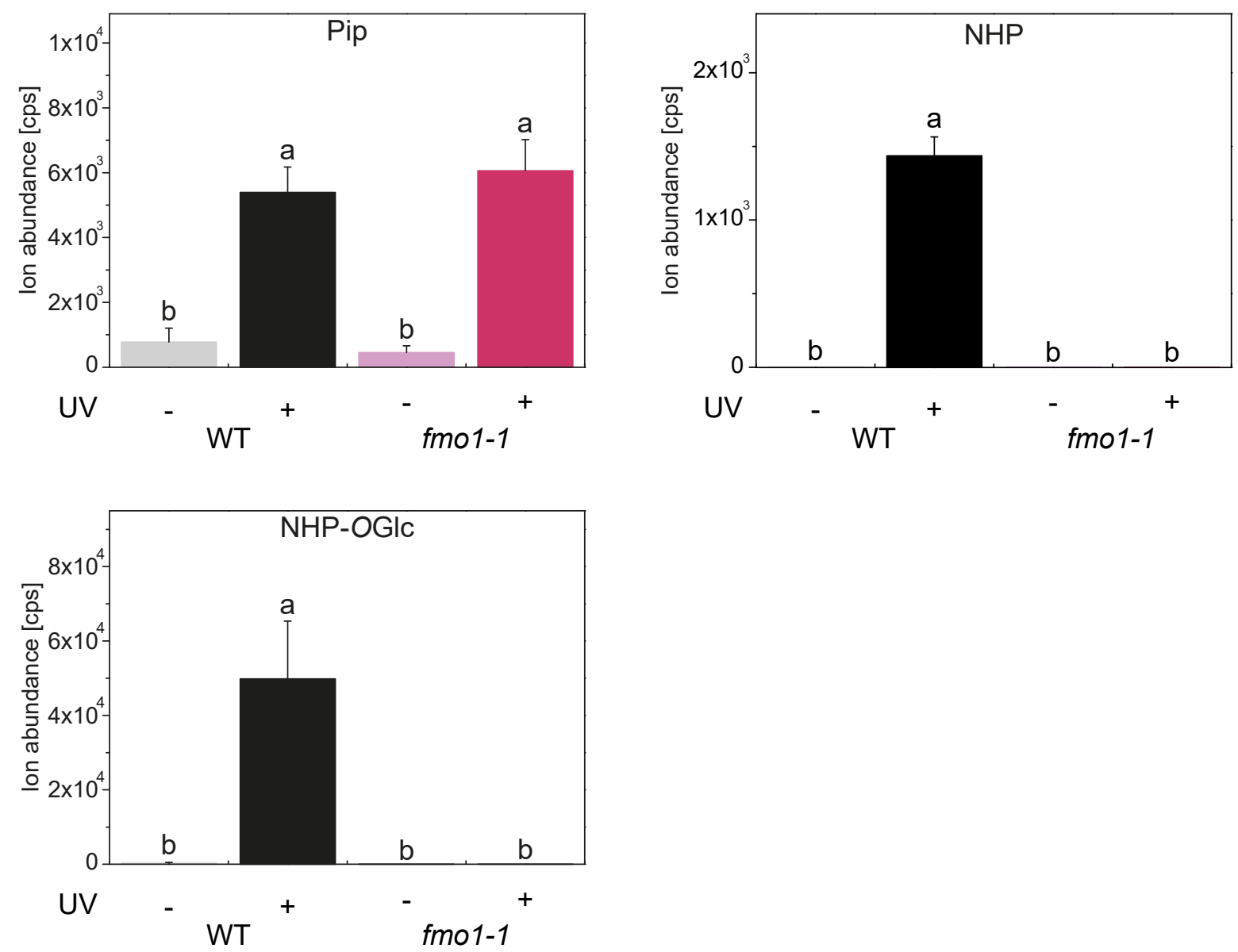

Supplementary Figure 2 UV-C induced NHP and NHP-OGlc biosynthesis is abolished in fmo1 mutant plants. Upon UV-C treatment, wild type plants produce substantial amounts of a, pipecolic acid (Pip), b, $\mathrm{N}$-hydroxy pipecolic acid (NHP) and c, N-hydroxy pipecolic acid glycoside (NHP-OGlc). As it was previously shown for pathogen induce Pip metabolism ${ }^{8}, f m o 1-1$ mutant plants are able to accumulate Pip in similar amounts as wild type plants, but are impaired in the biosynthesis of NHP and NHP-OGIc. Samples were collected as described in Figure 1. Bars represent the mean \pm STD of three biological replicates. Statistical differences among replicates are labeled with different letters $(P<0.05$, one-way ANOVA and post hoc Tukey's Test; $n=3$ ). The experiment was repeated twice with similar results. 


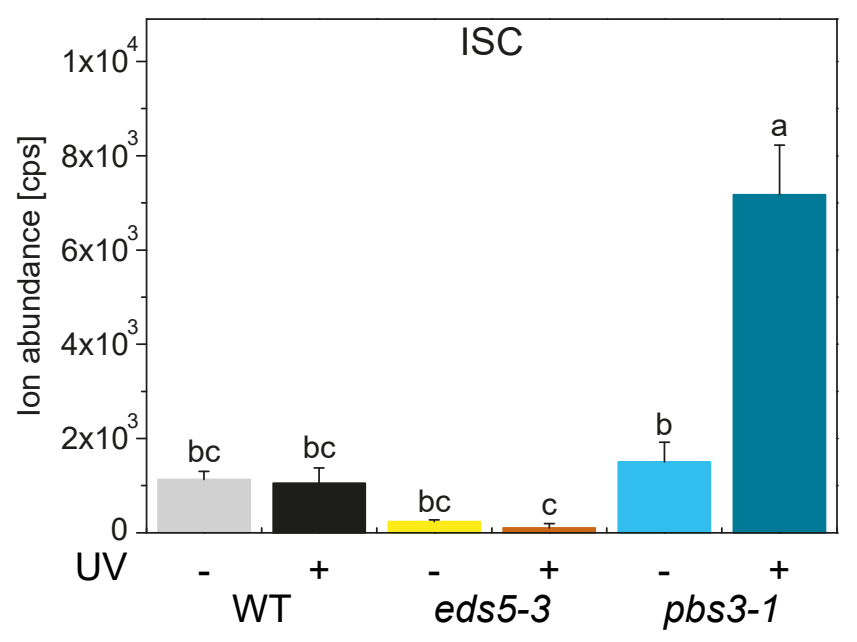

Supplementary Figure 3 UV-C treatment induce ISC accumulation in pbs3 mutant plants, related to

Figure 1. UV-C treatment is known to induce the formation of salicylic acid (SA) for a long time ${ }^{4}$. However, the accumulation of the SA precursor isochorismate (ISC) was not monitored so far. Here, we show that pbs3-1 mutant plants, but not wild type or eds5-3 mutant plants accumulate, accumulate substantial amounts of ISC. Samples were collected as described in Figure 1. Bars represent the mean \pm STD of three biological replicates. Statistical differences among replicates are labeled with different letters ( $P<0.05$, one-way ANOVA and post hoc Tukey's Test; $n=3)$. The experiment was repeated twice with similar results. 


\section{Methods}

Plant material. Arabidopsis plants were grown in a chamber at $22^{\circ} \mathrm{C}$ with a $16 \mathrm{~h}$ light period and $60 \%$ relative humidity for 4-5 weeks. For our experiments, we use Arabidopsis ecotype Col-0 and the following mutants in this background: eds $5-3^{4}, p b s 3-1^{18}$, sard4 $-5^{19}$, and fmo1- $1^{8}$, which were described previously.

UV-C and soil drench treatment. For the UV-C treatment, we followed previous protocol ${ }^{13}$. In short, 45 week old Arabidopsis plants were exposed to UV-C light $(254 \mathrm{~nm})$ for $20 \mathrm{~min}$ at $50 \mathrm{~cm}$ distance to the lamp (TUV T8 30W, Philips) for the induction of SA and Pip biosynthesis. For the treatment with SA respectively Pip, we followed previous protocols ${ }^{4,17}$. 4-5 week old plants, were soil drenched with either $10 \mathrm{~mL}$ water, or $10 \mathrm{~mL}$ of a $5 \mathrm{mM}$ Pip solution (P45850, Sigma) respectively $10 \mathrm{~mL}$ of a $5 \mathrm{mM} \mathrm{SA}$ solution (S5922, Sigma), equals to $50 \mu \mathrm{M}$ final concentration. Metabolites were extracted and analyzed, as described earlier ${ }^{19,20} 24$ hour after treatment. The NHP standard was chemical synthesized as described in Hartmann et al., 2018 . The MS/MS spectra of NHP and NHP-OGlc corresponds to the results from Chen et al., $2018^{9}$.

\section{Acknowledgments}

We are grateful to Ulf Diedrichsen and Brigitte Worbs for the synthesis of the NHP authentical standard. This work was supported by the DFG (IRTG 2172 "PRoTECT" program of the Göttingen Graduate Center of Neurosciences, Biophysics, and Molecular Biosciences.) to D.R., L.M. and I.F.; I.F. was additionally supported by DFG excellence initiative (ZUK 45/2010 and INST 186/822-1). Y.Z. was supported by Natural Sciences and Engineering Research Council of Canada, Canada Foundation for Innovation, and British Columbia Knowledge Development Fund. 


\section{Author contributions}

D.R., K.F. and I.F. conceived and designed the experiments. D.R., L. M., and K.Z. performed the experiments. D.R., K.F., Y. Z., and I.F. wrote the article. Competing interests: Authors declare no competing interests. Data and materials availability: All data is available in the main text or the supplementary materials. The authors responsible for distribution of materials integral to the findings presented in this article are: Ivo Feussner (ifeussn@uni-goettingen.de) and Yuelin Zhang (yuelin.zhang@ubc.ca).

\section{References}

1 Fu, Z. Q. \& Dong, X. Systemic acquired resistance: Turning local infection into global defense. Annu. Rev. Plant Biol. 64, 839-863, doi:10.1146/annurev-arplant-042811-105606 (2013).

2 Bernsdorff, F. et al. Pipecolic acid orchestrates plant systemic acquired resistance and defense priming via salicylic acid-dependent and -independent pathways. Plant Cell 28, 102129, doi:10.1105/tpc.15.00496 (2016).

3 Garcion, C. et al. Characterization and biological function of the ISOCHORISMATE SYNTHASE2 gene of Arabidopsis. Plant Physiol. 147, 1279-1287, doi:10.1104/pp.108.119420 (2008).

$4 \quad$ Nawrath, C. EDS5, an essential component of salicylic acid-dependent signaling for disease resistance in Arabidopsis, is a member of the MATE transporter family. Plant Cell 14, 275-286, doi:10.1105/tpc.010376 (2002).

5 Jagadeeswaran, G. et al. Arabidopsis GH3-LIKE DEFENSE GENE1 is required for accumulation of salicylic acid, activation of defense responses and resistance to Pseudomonas syringae. Plant J. 51, 234-246, doi:10.1111/j.1365-313X.2007.03130.x (2007).

6 Song, J. T., Lu, H. \& Greenberg, J. T. Divergent roles in Arabidopsis thaliana development and defense of two homologous genes, ABERRANT GROWTH AND DEATH2 and AGD2-LIKE DEFENSE RESPONSE PROTEIN1, encoding novel aminotransferases. Plant Cell 16, 353-366, doi:10.1105/tpc.019372 (2004).

7 Sharma, S., Shinde, S. \& Verslues, P. E. Functional characterization of an ornithine cyclodeaminase-like protein of Arabidopsis thaliana. BMC Plant Biol 13, 182, doi:10.1186/1471-2229-13-182 (2013).

8 Hartmann, M. et al. Flavin monooxygenase-generated N-hydroxypipecolic acid is a critical element of plant systemic immunity. Cell, doi:10.1016/j.cell.2018.02.049 (2018).

9 Chen, Y.-C. et al. $N$-hydroxy-pipecolic acid is a mobile metabolite that induces systemic disease resistance in Arabidopsis. Proc. Natl. Acad. Sci. USA 115, E4920-E4929, doi:10.1073/pnas.1805291115 (2018).

10 Zhang, M. \& Robertus, J. D. Molecular cloning and characterization of a full-length flavindependent monooxygenase from yeast. Arch. Biochem. Biophys. 403, 277-283, doi.org/10.1016/S0003-9861(02)00237-0 (2002).

11 Song, J. T. Induction of a salicylic acid glucosyltransferase, AtSGT1, is an early disease response in Arabidopsis thaliana. Mol. Cells 22, 233-238 (2006). 
12 Li, Y., Baldauf, S., Lim, E.-K. \& Bowles, D. J. Phylogenetic analysis of the UDP-glycosyltransferase multigene family of Arabidopsis thaliana. J. Biol. Chem. 276, 4338-4343, doi:10.1074/jbc.M007447200 (2001).

13 Serrano, M. et al. Export of salicylic acid from the chloroplast requires the multidrug and toxin extrusion-like transporter EDS5. Plant Physiol. 162, 1815-1821, doi:10.1104/pp.113.218156 (2013).

14 Widhalm, J. R. et al. Identification of a plastidial phenylalanine exporter that influences flux distribution through the phenylalanine biosynthetic network. Nat. Commun. 6, 8142, doi:10.1038/ncomms9142 (2015).

15 Kirma, M., Araújo, W. L., Fernie, A. R. \& Galili, G. The multifaceted role of aspartate-family amino acids in plant metabolism. J. Exp. Bot. 63, 4995-5001, doi:10.1093/jxb/ers119 (2012).

16 Chang, M. et al. PBS3 protects EDS1 from proteasome-mediated degradation in plant immunity. Mol. Plant, doi:10.1016/j.molp.2019.01.023 (2019).

17 Navarova, H., Bernsdorff, F., Döring, A.-C. \& Zeier, J. Pipecolic acid, an endogenous mediator of defense amplification and priming, is a critical regulator of inducible plant immunity. Plant Cell 24, 5123-5141, doi:10.1105/tpc.112.103564 (2012).

18 Nobuta, K. et al. The GH3 acyl adenylase family member PBS3 regulates salicylic aciddependent defense responses in Arabidopsis. Plant Physiol. 144, 1144-1156, doi:10.1104/pp.107.097691 (2007).

19 Ding, P. et al. Characterization of a pipecolic acid biosynthesis pathway required for systemic acquired resistance. Plant Cell 28, 2603-2615, doi:10.1105/tpc.16.00486 (2016).

20 Kusch, S. et al. Arabidopsis mlo3 mutant plants exhibit spontaneous callose deposition and signs of early leaf senescence. bioRxiv, 558122, doi:10.1101/558122 (2019). 


\section{Discussion}

Upon infections, plants undergo changes in their transcriptome and metabolome. Additionally, chromatin remodeling as well as histone modification allow a rapid response to the actual pathogen, but also prepare to set-up distal organs against subsequent threats. The expression of defense related genes, translation into the corresponding proteins and the subsequent production of signaling metabolites are the key events for a successful activation of the plant immunity. Thereby, the precious spatiotemporal accumulation of signaling compounds, like NHP and SA, in local and distal tissues is crucial to orchestrate all processes involved in the plant immune system. Thus, the biosynthesis and catabolism of signaling compounds must be regulated strictly. A mutation in genes that are involved in the homeostasis of signaling compounds often leads to enhanced pathogen susceptibility. This holds true for the four genes, which were studied in this thesis, namely ALD1, EDS5, PBS3 and SARD4. In order to understand their molecular role in the establishment of SAR, metabolite fingerprint analyses upon pathogen infection (Article I) or UV-C treatment (Article III) were performed. Beside external stresses, the utilization of autoimmunity mutants was instrumental to solve the old question of SA biosynthesis (Article II).

The basis for the results presented in this thesis was always a comprehensive untargeted metabolite fingerprint analysis. The assumption for this work was that upon a specific stress, the metabolic machinery responds by metabolic changes. A mutation in a gene encoding for an enzymatically active protein should lead to the accumulation of the substrate and the absence of the corresponding product. This hypothesis was instrumental to identify the function of SARD4 (Article I) and PBS3 (Article II) in the Pip respectively SA biosynthesis. The situation became more complicated, when the plastidial transporter EDS5 was investigated. In analogy to the other proteins studied in this thesis, it was expected that the cargo would accumulate upon stress treatment. However, only the absence of the downstream products could be detected, but no substrate accumulation.

The knowledge derived from the untargeted metabolomics studies was subsequently used to examine the activity of the corresponding enzymes in vitro. A particular challenge in these investigations was the lack of commercially available substrates for the activity assays of SARD4 and PBS3, namely P2C and ISC. Therefore, it became essential to heterologously express enzymes, which act upstream in these metabolic pathways, to 
produce substrates for SARD4 respectively PBS3 enzymatically. The results discussed in the upcoming sections could answer some of the longstanding questions in the research area of plant immunity.

\subsection{Pip biosynthesis in systemic leaves depends on SARD4}

Even though the presence of Pip in plants has been known for decades (Zacharius et al., 1952; Morrison, 1953), its function stayed elusive. Two major routes for the biosynthesis were proposed (see section 1.4), although the enzymes in these pathways were not characterized. In 2004, the Greenberg group published two reports that described the so far unknown aminotransferase ALD1 (Song et al., 2004a; Song et al., 2004b). They observed that ALD1 transcripts are upregulated upon Pseudomonas infection and that ald1 mutant plants show enhanced susceptibility for this pathogen. ALD1 was then heterologously expressed in E. coli, purified and its in vitro activity was subsequently examined. Song and colleagues observed a substrate specificity for lysine (Song et al., 2004a). This group could not identify the precise pathway, in which ALD1 is involved. They concluded however that there must be an amino acid derived signal, which on the one hand stimulates the biosynthesis of SA and on the other hand modulates the plant immunity independently from SA (Song et al., 2004b). Additionally, they not only could show that ald1 mutant plants are affected in their basal resistance, but also that they fail to establish SAR. When Arabidopsis plants were analyzed for alterations of their free amino acids upon infection, Navarova and colleagues found that Pip and $\alpha$-aminoadipate accumulated locally and systemically. When they infected mutant plants, which are known for their deficiencies in plant immunity, they found that ald1 plants were not able to synthesize Pip (Navarova et al., 2012). Moreover, external Pip could recover the resistance of ald1 plants against Pseudomonas infections and reestablish SAR as well. Therefore, it was concluded that the phenotype of ald1 plants is due to the inability to synthesize Pip and that ALD1 is responsible for the first step of Pip biosynthesis. On the other hand, the report shows that in local, but not in systemic, leaves of fmo1 plants, Pip accumulates in higher amounts than in wild type plants upon infection. External Pip could not recover the susceptibility phenotype of fmo1 plants. This suggests that FMO1 is required to modify Pip in order to fully activate Pip signaling, which is then crucial for basal immunity and SAR. 
ALD1 is a monofunctional aminotransferase and thus, there was a need for a reductase, which is able to catalyze the final step of Pip biosynthesis (Zeier, 2013). In mammals, this reaction is catalyzed by the ketimine reductase $\mu$-crystallin (CRYM), where it is involved in hormone biosynthesis, brain development and is present in retinae of different species (Hallen et al., 2015). The closest homology to the human CRYM can be found in the Arabidopsis gene At5g52810 (30.5\% amino acid identity, $51.8 \%$ amino acid similarity, (Zeier, 2013)). This gene was annotated as an ornithine cycloadeaminase for its similarity to the bacterial ornithine cycloadeaminase, which converts ornithine into proline. When Sharma and colleagues studied this gene, they found no significant alterations in proline levels in either the mutant or the overexpression plants. Additionally, in vitro assays did not show any activity towards ornithine (Sharma et al., 2013).

In order to identify new key genes involved in the establishment of SAR, the Zhang group developed the high throughput "brush and spray" method (Jing et al., 2011). Two of the mutants in this screen had a mutation in the previously mentioned At5g52810 gene. When these mutants were studied in more detail, the SAR deficiency phenotype could be clearly confirmed and thus this gene was named SAR Deficient 4 (SARD4). When sard4 was crossed with the hyper-resistant FM01 overexpression line (Koch et al., 2006), the enhanced resistance was reverted again. This was the first hint that SARD4 and FM01 might be active in the same pathway. In Article 1, the amount of Pip in sard4 mutant plants upon infection was assessed. It was found that in infected leaves Pip was significantly reduced, but still measureable, whereas distal tissues of sard4 mutant plants failed to synthesize detectable amounts of Pip.

In this work, we tested the hypothesis on the function of SARD4 by an untargeted metabolite fingerprint analysis with distal leaves of plants, which were previously infected with Pseudomonas. Exactly one signal was identified in the untargeted approach, which followed our general assumption for a tentative SARD4-substrate: to be enriched in sard4 - but not ald1 mutant plants. By data base search and MS/MS fragmentation experiments we could identify P2C as tentative Pip precursor. Pip itself was absent in the systemic leaves of both mutant lines. To confirm this finding from the metabolomics approach, the AtALD1 and AtSARD4 genes were amplified and expressed in E.coli. Cultures of $E$. coli harboring either of these genes individually did not produce detectable amounts of Pip. However, when both genes were expressed in one culture simultaneously, the formation of Pip could be observed. This means that the Arabidopsis Pip biosynthesis could be reconstructed completely in E. coli. To study the activity of SARD4 also 
biochemically in a cell free approach and therefore, to show a third way of evidence, we purified the E. coli produced SARD4 protein. The tentative substrate of SARD4, P2C, however, is commercially not available and thus, purified ALD1 was employed in order to produce P2C from lysine. Additionally, utilizing $\left[6-{ }^{13} \mathrm{C},-15 \mathrm{~N}\right]-\mathrm{L}-\mathrm{lysine}$ as starting material, $\left[6-{ }^{13} \mathrm{C},{ }^{15} \mathrm{~N}\right]$-Pip could be produced in a sequential reaction of ALD1 and SARD4. Thus, it was concluded that SARD4 is indeed the missing ketimine reductase in the pathogen triggered biosynthesis of Pip in Arabidopsis (Figure 4). Additionally, the application of labeled lysine allowed an unambiguous identification of $\mathrm{P} 2 \mathrm{C}$, and not $\mathrm{P} 6 \mathrm{C}$, as the intermediate of this pathway. Therefore, one of the two previously proposed pathogeninduced Pip biosynthesis pathways (Zeier, 2013) could be confirmed by our work (Article I). An independent group validated the biological role and function of SARD4 within the establishment of SAR (Hartmann et al., 2017). In this study, the researcher observed, utilizing a GC coupled to Fourier-transform infrared spectroscopy, that P2C tautomerizes in solution to its enamine form. However, the conclusion that the enamine is the final product of the ALD1 reaction over-interprets the data. The tautomerism between the enamine and ketimine form of P2C has been known for a long time and was shown to be strongly dependent on the $\mathrm{pH}$ value of the solution. The imine form was shown to be the major form at neutral $(\mathrm{pH}=7.4)$ and hence physiological conditions, while the enamine form occurs only in strong basic solutions (Lu and Lewin, 1998). Moreover, in infrared spectroscopy, tertiary amines cannot be monitored (Dr. Lucas Busta personal communication). As the characteristic $\mathrm{N}=\mathrm{C}$ double bond of the ketimine form cannot be found by this method, the tautomerization state cannot be identified unambiguously. In 2018, $\mathrm{Xu}$ and colleagues reported that they found $A L D 1$ and SARD4 homologs in the lycopod Huperzia serrata. Heterologously expressed and purified HsALD1and HsSARD4 were studied for their ability to catalyze the same reactions as shown with the Arabidopsis enzymes. Thereby, unequivocal data were presented showing that only the ketimine form of P2C is the substrate of HsSARD4 (Xu et al., 2018). Taking these results together, it is very likely that AtSARD4 is a ketimine reductase, which does not convert the enamine form into Pip.

An important aspect of Article I and the report from Hartmann et al., 2017 is that upon infection of sard4 plants, the accumulation of Pip in affected tissues is only reduced, but not abolished. In systemic leaves of sard4, however, no Pip can be detected at all. It seems conceivable that there might be a second reductase in Arabidopsis, which is only active in the infected but not in systemic leaves. Another, more speculative, explanation might be 
that the reduction of $\mathrm{P} 2 \mathrm{C}$ into Pip occurs spontaneously. As mentioned briefly in section 1.2, it is known that disulfide bridges of cytosolic NPR1 oligomers are spontaneously reduced upon infection due to redox changes (Mou et al., 2003). It might be possible that this redox change is already sufficient to reduce P2C into Pip nonenzymatically, as it has been shown before for other imines (Potter and Hinson, 1986).

The influence of SARD4 in basal resistance in general and a possible difference between sard4 and ald1 mutant plants in particular is an ongoing debate. We could show in our work (Article I) that no enhanced susceptibility of sard4 plants towards Pseudomonas infection was detectable. In the report of Hartmann and colleagues from 2017, the basal resistance of sard4 mutants was significantly attenuated. In sard4 plants, wild type like levels of SA accumulate in affected leaves upon infection (Article I, (Hartmann et al., 2017)), whereas SA levels are significantly reduced in infected ald1 leaves (Song et al., 2004a). Upon external application of SA, the basal resistance in ald1 plants is strongly enhanced (Bernsdorff et al., 2016). Therefore, it is conceivable that there is a difference in basal resistance between ald1 and sard4 plants and that this difference is due to disparate levels of SA accumulation.

\section{2. $\mathrm{PBS}_{3}$ is the yet missing link in the SA biosynthesis}

As outlined in section 1.3., two routes for the biosynthesis of SA were proposed. Despite extensive efforts, neither of the pathways could be completely uncovered so far (Klessig et al., 2018). In the ISC pathway, the major route of SA biosynthesis in Arabidopsis (Garcion et al., 2008), up to now only ICS1 was identified as part of the pathway. Moreover, no gene with sufficiently high homology to bacterial IPL, which would catalyze the elimination reaction of ISC into SA and pyruvate, could be found in the plant genome (Dempsey et al., 2011). Furthermore, a biosensor screen did not detect an IPL enzyme activity in the cDNA library of Arabidopsis (Zhou et al., 2018). Therefore, it is conceivable that plants use a different mechanism than bacteria in order to convert ISC into SA. In addition to ics 1 several other Arabidopsis mutant lines were previously identified, which are impaired in SA accumulation upon infection (e.g. eds5 (Nawrath, 2002), eps1 (Zheng et al., 2009)). For the pbs3 mutant plants slightly different results, in regard to SA respectively SAG accumulation, were reported. Nobuto and colleagues could still detect residual SA but no SAG accumulation upon infection in pbs3 plants, whereas in 
Jagadeeswaran et al., neither SA nor SAG were found (Jagadeeswaran et al., 2007; Nobuta et al., 2007). A possible explanation for this discrepancy comes from a very recent report, in which it was shown that $P B S 3$ is regulated differently in leaves of different age (Berens et al., 2019). Therefore, it is conceivable that analysis of leaves of different age is responsible for the divergent results. However, in both reports from 2007 exogenous application of SA could recover the enhanced pathogen susceptibility of pbs3 mutants. Thus, the interaction of PBS3 with the SA biosynthesis/signaling pathway was accepted, although not understood. PBS3 belongs to the widespread GH3 family of acyl acid amido synthetases (Yu et al., 2018). In plants, SA-aspartate is the only SA-amino acid conjugate known so far. However, the concentration of this compound is very low, and its function is debatable (Zhang et al., 2007). Therefore, it is not surprising that when the biochemical activity of PBS3 was investigated, SA was not a suitable substrate. Instead, SA acted in vitro as a potent inhibitor for the PBS3-catalyzed reaction of p-aminobenzoic acid with the amino acid glutamate (Okrent et al., 2009). Despite this comprehensive study from the Wildermuth laboratory, the connection between PBS3 and SA stayed elusive.

The crucial information about the tentative function of PBS3 came from a metabolite fingerprint analysis of several Arabidopsis mutants in the autoimmunity background termed suppressor of npr1-1, constitutive 2 (snc2, (Zhang et al., 2010a)). NPR1 is a master regulator of SA dependent plant immunity (Ding et al., 2018). As depicted in Article II, snc2npr1 plants accumulate high amounts of SA and SAG without the need of an external induction. This accumulation is abolished when PBS3 or EDS5 are mutated additionally in these plants. In the snc2npr1pbs3 but not in the snc2npr1eds5 plants, however, an accumulation of ISC could be observed. Following, once again, the general assumption of this work (precursor accumulation of the blocked enzymatic step due to a mutation in the corresponding gene), it was concluded that ISC might be the endogenous substrate of PBS3 with SA as the final product. To verify this hypothesis, PBS3 was cloned, heterologously expressed and subsequently purified to homogeneity. As ISC is commercially not available, ICS1 was also purified in order to catalyze the isomerization of CA into ISC. PBS3 was able to utilize both compounds, CA and ISC, as acyl substrate for the conjugation with Glu with very high conversion rates. However, ISC seems to be the preferred substrate of PBS3. CA and ISC possess two carboxy groups each (at C7 respectively (9), which both could be a target for the conjugation with Glu. Tandem mass spectrometry unambiguously revealed that ISC-9-Glu is the predominant product of the PBS3 catalyzed reaction. Beside ISC-9-Glu, two more compounds in the reaction became 
apparent in the mass spectrometry analysis, namely SA and 2-hydroxyacryloyl- $N$ glutamic acid (2HNG), which can be added up to the corresponding mass of ISC-9-Glu. To verify that the synthesis of SA depends on the enzymatic activity of PBS3, individual components of the full reaction assay (PBS3, Glu, ATP, ISC) were left out individually. The amount of SA formed within an hour was four times higher in the full reaction assay compared to when any of the individual components PBS3, Glu, or ATP were missing. This finding supports the idea that PBS3 is indeed directly responsible for the synthesis of SA. As no lyase function of a GH3 enzyme was reported so far, the stability of ISC-9-Glu and ISC and the occurrence of SA was monitored with mass spectrometry for 24 hours. It was observed that ISC-9-Glu decays over time without the requirement of an additional enzyme to give rise to SA. In comparison to the non-enzymatic decay of ISC, which was reported previously (DeClue et al., 2006), the decay of ISC-9-Glu was ten times faster. From these results we deduced that PBS3 derived ISC-9-Glu, which decays nonenzymatically in solution to SA and 2HNG, is the missing link between ISC and SA.

In order to confirm the preference of PBS3 towards ISC, enzyme kinetics were studied. In the comprehensive report from Okrent and colleagues, the highest catalytical efficiency of PBS3 was achieved, when 4HBA was conjugated with Glu (Okrent et al., 2009). We could show that the catalytic efficiency was 740 times higher, when ISC was used as a substrate instead of 4HBA (Article II). Our results strengthen the hypothesis that ISC is indeed the endogenous substrate of PBS3. The physiological impact of these results became apparent, when the data of the untargeted metabolomics approach from snc2npr1, snc2npr1pbs3 and snc2npr1eds5 plants were reanalyzed. Similar to SA and SAG, ISC-9-Glu and 2HNG were present in the plant material from snc2npr1 but not in snc2npr1pbs3 or snc2npr1eds5. As the MS/MS spectra of in vitro as well as in planta derived ISC-9-Glu were nearly identical, it was concluded that the reaction identified and characterized in vitro occurs likewise in plants.

Previously, it was shown that ICS1, which provides the substrate ISC for the identified PBS3 reaction, is located in chloroplasts (Strawn et al., 2007). As PBS3 does not possess a plastidial signal peptide, the spatial distribution of the proposed pathway required a thorough examination. Therefore, different fluorophores (such as yellow fluorescence protein (YFP) and cyano fluorescence protein (CFP)) were combined with ICS1, PBS3 and a PBS3 construct that carried the N-terminal signal peptide of ICS1. When these constructs were expressed transiently in Arabidopsis leaves of efr mutant plants, the PBS3-YFP construct could be detected in the cytosol, whereas ICS1-CFP and plastidial PBS3-YFP 
(chloroPBS3-YFP) co-localized in chloroplasts when expressed within the same leaf. The Arabidopsis efr mutants are suitable for Agrobacterium tumefaciens mediated transient expression in Arabidopsis, as this mutant lacks a receptor, which activates MTI in wildtype plants upon contact with A. tumefaciens (Zipfel et al., 2006). These data confirmed the previous reports that ICS1 is located in chloroplasts (Strawn et al., 2007) and the recent report of cytosolic localization for PBS3 (Chang et al., 2019). For the proposed pathway, this means that ICS1 catalyzes the conversion of CA into ISC in plastids, which is subsequently exported into the cytosol where PBS3 catalyzes the conjugation with Glu and the non-enzymatic decay to SA and 2HNG takes place. This model contradicts previous reports, which proposed that SA is exclusively produced in plastids (Fragniere et al., 2011). Three major reasons can be found in the literature for the assumption that SA is synthesized in plastids: 1. ICS1 and ICS2 are both present in plastids (Garcion et al., 2008); 2. When the plastidial MATE-like transporter EDS5 is knocked out, no SA accumulation can be measured upon infection in the eds5 mutant plants (Serrano et al., 2013); 3. When the bacterial SA hydroxylase gene NahG is expressed in chloroplasts of Arabidopsis leaves, no SA can be detected upon infection with Pseudomonas (Fragniere et al., 2011). Reason 1 corresponds to the model, which we provide in Article II. The same is true for reason 2, if EDS5 exports ISC instead of SA. In order to verify this assumption, chloroPBS3-YFP was transiently expressed alone and together with ICS1-CFP in eds5 mutant plants. Indeed, the co-expression of ICS1-CFP and chloroPBS-YFP in the eds5 plants enabled a significant accumulation of SA, whereas ICS1-CFP together with cytosolic PBS3-YFP did not lead to detectable amounts of SA. Therefore, it is conceivable that EDS5 is indeed responsible for the export of ISC, which needs to be transferred through the plastidial membrane in order to be accessible for PBS3. This result is in agreement with reason 2 from above. Moreover, it could explain, why others and we did not observe an SA accumulation in eds5 plants. CA is described as an important metabolic node in the biosynthesis of aromatic amino acids (Maeda and Dudareva, 2012). Therefore, it is conceivable that ISC, which cannot be exported out of the plastids in eds5 plants, is converted back into CA, as the ICS1 reaction operates nearly at CA/ISC equilibrium (Strawn et al., 2007), which subsequently feeds into the synthesis of other metabolites like aromatic amino acids. Consequently, no SA and especially no ISC accumulation can be observed in this mutant. This explanation is more convincing than the one presented in the report from Serrano and colleagues, where it was proposed that SA inhibits the ICS1 reaction (Serrano et al., 2013). This hypothesis was not supported by experimental data 
and contradicts the observation of the positive feedback from SA and ICS1 gene expression (Hunter et al., 2013).

The remaining argument that the expression of NahG in chloroplasts abolishes the SA biosynthesis (Fragniere et al., 2011), is contradictory only, if NahG exclusively accepts SA as its substrate. The Pseudomonas putida derived NahG was often used in plant science as a SA hydroxylase that converts SA into catechol in order to present the importance of SA for plant immunity (Gaffney et al., 1993). However, the substrate specificity of NahG was not questioned in these reports. Interestingly, when eds5 and sid2 plants were studied comprehensively a strongly reduced camalexin accumulation upon infection could be identified when NahG was expressed in these plants. Therefore, it was concluded that NahG might act on pathways other than just SA (Heck et al., 2003). Zhao and colleagues were able to heterologously express NahG in E. coli. Subsequent purification allowed to study this protein in vitro. Although only derivatives of SA were tested in this report, it becomes clear that NahG not only accepts substrates other than SA but shows also enhanced activity for the hydroxylation of sulfonated and methylated SA (Zhao et al., 2005). In the report from Fragnier et al., the authors are admitting that the cytosolic expression of NahG shows a stronger effect on SA depletion than expression in chloroplasts. The authors speculate that this difference is due to an additional, minor site for the synthesis of SA other than chloroplasts (Fragniere et al., 2011). Taking the results from Heck and Zhao into account, another explanation can be provided. It is conceivable that NahG, when expressed in chloroplasts, could hydroxylate the ICS1 derived ISC, which would consequently lead to a reduced accumulation of SA as observed by Fragnier and colleagues. This reduction is weaker when NahG is expressed in chloroplasts, as low amounts of ISC might still be exported by EDS5 from the chloroplasts into the cytosol. On the other hand, when NahG is overexpressed in the cytosol, it can hydroxylate the PBS3 derived SA and/or plastidial derived ISC. Consequently, the amount of SA is stronger reduced, when NahG is overexpressed in the cytosol than in the chloroplasts. Taken together, all three reasons for the assumption that SA biosynthesis is located in the plastids can be interpreted in a different way in light of the findings described in article II. Moreover, the underlying experimental results, which led to those postulations, could be incorporated into the working model without contradictions (Article II).

Which physiological advantage might a non-enzymatic decay have over an enzymatically regulated process, as known from bacteria? One important aspect of plant immunity is the tradeoff between defense and growth (Martinez-Medina et al., 2016). A rushed 
accumulation of SA might induce HR before all required metabolic allocations are completed (Bolton, 2009). The fast production of a depot-compound, which delays the activation of HR, would bypass this problem. This interim step in the biosynthesis of SA would also explain, why the expression of ICS1 (Hunter et al., 2013), EDS5 (Nawrath, 2002) and PBS3 (Nobuta et al., 2007) reaches its peak several hours before an increase in SA can be observed. Indeed, when the time courses of the accumulation of ISC-9-Glu and SAG upon UV-C treatment were compared, it was observed that ISC-9-Glu reaches its maximum 8 hours, whereas the maximum for SAG was observed 24 hours after UV-C stress (Supplementary Figure 1, (Nawrath, 2002)). The fast utilization of CA might be an important strategy as this compound seems to be a preferred target of several pathogenic effectors. The pathogenic fungus Ustilago maydis, responsible for the corn smut disease, secretes an effector called Cmu1 into corn, which has a proposed chorismate mutase activity (see Figure 3). When corn plants were infected with $U$. maydis lacking the CMU1 gene, they accumulated SA significantly higher than upon infection with the wild type fungus (Djamei et al., 2011). It was proposed that this effector converts CA into prephenate and thereby channels CA away from the SA biosynthesis. Interestingly, Cmu1 does not contain a plastidial signal peptide and consequently is present only in the cytosol but not in the plastids of the infected plants. Due to the known substrate promiscuity of CA utilizing enzymes (Luo et al., 2011) and considering the results presented in Article II, it is conceivable that Cmu1 targets ISC instead of CA in infected plants and thereby prevents the biosynthesis of SA. Two other effectors are known to target ISC in the cytosol of infected plants to prevent SA formation. Liu and colleagues showed that the oomycete Phytophthora sojae and the fungus Verticillium dahliae both contain effectors that possess an isochorismatase activity. Both enzymes converted ISC into 2,3-dihydro-2,3-dihydroxy benzoate (DDHB) and pyruvate. Again, these effectors were present only in the cytosol and could not be detected in plastids (Liu et al., 2014). Therefore, it appears to be crucial for plants to channel CA into SA rapidly, before pathogen derived effectors might interfere with these metabolites. A prerequisite for that rapid channeling might be the very high affinity of PBS3 towards ISC. In comparison with other GH3 enzymes, e.g. OsGH3.8 with $K_{\mathrm{m}}^{\mathrm{IAA}}=182 \mu \mathrm{M}$ and $\mathrm{V} / \mathrm{E}_{\mathrm{t}}^{\mathrm{IAA}}=30.1 \mathrm{~min}^{-1}$ (Chen et al., 2010), AtGH3.11 with $K_{\mathrm{m}}^{\mathrm{JA}}=351 \mu \mathrm{M}$ and $V / E_{\mathrm{t}}{ }^{\mathrm{A}}=24.8 \mathrm{~min}^{-1}$ (Westfall et al., 2012) or AtGH3.15 with $K_{\mathrm{m}}^{\mathrm{IBA}}=527 \mu \mathrm{M}$ and $\mathrm{V} / \mathrm{E}_{\mathrm{t}}^{\mathrm{IBA}}=9.9 \mathrm{~min}^{-1}$ (Sherp et al., 2018), PBS3 has the highest substrate specificity and the fastest turn-over number among the investigated GH3 enzymes with $K_{\mathrm{m}}{ }^{\mathrm{ISC}}=14.9 \mu \mathrm{M}$ and $\mathrm{V} / \mathrm{E}_{\mathrm{t}} \mathrm{ISC}=472.3 \mathrm{~min}^{-1}$. This strongly suggests that the reaction takes place rapidly upon the 
accumulation of ISC in the cytosol. Therefore, the spatial separation of ICS1 and PBS3 seems to be an important regulatory element. Another mechanism to prevent hyperaccumulation of SA seems to be the feedback inhibition towards PBS3 (Okrent et al., 2009). Thereby, ISC-9-Glu released SA can act as a competitive inhibitor, as depicted in Article II. In the previously published crystal structure of PBS3, co-crystalized with SA, it is apparent that SA can effectively bind at the active site of PBS3 (Westfall et al., 2012) and thereby prevent the binding of another acyl substrate. Utilizing the same crystal structure, a docking model in Article II could show that ISC fits into the active site of PBS3 and thereby, the C9-carboxy groups appears in the immediate proximity to AMP, which is required for the activation of the acyl substrates in GH3 enzymes.

Another advantage of a non-enzymatic decay mechanism for SA biosynthesis might be the previously mentioned substrate promiscuity of CA utilizing enzymes. The same enzyme might possess a lyase and mutase activity alike (Marti et al., 2009). Enzymatic reactions end up in an equilibrium between substrate and product, as observed for the ICS1 reaction (Strawn et al., 2007). This equilibrium might be exploited by pathogens, e.g. reduction of the substrate by a competing enzyme would enhance the reverse reaction. On the other hand, the non-enzymatic process has some advantages (Keller et al., 2015). The non-enzymatic decay is specific and one directional. Therefore, it is prone to site reactions and equilibrium interferences. Key enzymes are often targeted by pathogenderived modifications and metabolites in order to interfere with the immune response. A prominent example is the Pseudomonas derived Ja-Ile mimic coronatine. Virulent Pseudomonas strains use coronatine to activate JA-Ile signaling, which consequently suppresses SA-signaling (Katsir et al., 2008). Such an interference of a pathogen-derived metabolite with a non-enzymatic process is hardly conceivable.

Taken all together, it was shown by our work that there is an alternative strategy to the previously reported bacterial IPL enzymes for the biosynthesis of SA. As Article II is the first report to describe the SA biosynthesis as a chemical decay from a labile precursor, it seems probable that this process will be also found in other species. Given the widespread distribution of GH3 and ICS genes throughout the plant kingdom (Yokoo et al., 2018; Yu et al., 2018), it is conceivable that this pathway is conserved among a majority of plants. Additionally, the number of characterized bacterial IPLs respectively one-step SA synthases is limited. The discovery of a new possibility to produce SA in planta might enable the identification of similar pathways in bacteria. Indeed, not only ICS homologs are known from bacteria, but also bacterial genes that show significant similarities to GH3 
genes can be found in microbes (Johansen et al., 2018). Bacteria use SA as an intermediate in their siderophore biosynthesis, which in turn are important for their virulence (Lamb, 2015). Therefore, it is tempting to speculate that results presented in Article II might not only help to control phytopathogens but also to fight against human infections.

\section{3. $\quad$ EDS $_{5}$ - gatekeeper in SA and NHP biosynthesis alike}

Chloroplasts are mostly known for their key role in photosynthesis. On the other hand, the biosynthesis of different plant signaling compounds, such as JA-Ile (Wasternack and Strnad, 2019), SA (Dempsey et al., 2011), abscisic acid (ABA, (Finkelstein, 2013)) and the ethylene precursor methionine (Ravanel et al., 2004), occurs at least partially in chloroplasts. It is therefore not surprising that plastids are essential hubs for a successful defense against plant pathogens (Lu and Yao, 2018). For SA biosynthesis, the spatial distribution was discussed in section 5.2. Here, the spatial distribution of the enzymatic steps required for the biosynthesis of another signal molecule NHP, the FMO1 catalyzed follow-up product of Pip, should be reviewed. For ALD1 and SARD4, the two enzymes, which are responsible for the biosynthesis of Pip, it was shown previously that they are active in plastids (Song et al., 2004a; Sharma et al., 2013; Jung et al., 2016). The localization of FM01 however stayed elusive so far. A growing body of evidence points towards a localization at the endoplasmic reticulum (ER). Although neither a transmembrane nor a membrane anchor domain can is present in the FMO1 gene, studies on other FMOs showed a localization at the ER surface (Zhang and Robertus, 2002). In addition to the ER localization, some FMOs might be also present in the cytosol (Kriechbaumer et al., 2016). Interestingly, Li and colleagues observed that especially the overexpression of FMOs leads to their accumulation in the ER. When the authors added cycloheximide as translation inhibitor, the fluorescence signal in the ER vanished and in turn appeared in the cytosol (Li et al., 2010). Therefore, the precise allocation of FM01 might be difficult. However, results from the Zeier group strongly suggest that FMO1 is indeed localized at the ER (Bernsdorff, 2014). No matter whether FM01 is located in the cytosol or at the ER, in both cases Pip would need to be transported out of plastids first in order to be $N$-hydroxylated by FMO1 to yield NHP (Hartmann and Zeier, 2018).

In order to examine the role of a protein that is related to plant immunity, mutant plants, defective in the encoding gene, are often challenged by an appropriate pathogen. 
However, plant infection studies depend on the plant fitness as well as on the ability of the pathogens to successfully induce the plant immunity. Biotic and abiotic factors might influence the properties of plants and pathogens alike and consequently interfere with the outcome of an experiment. Without questioning the results or evaluating their validity, the expression data presented in the report from Bernsdorff and colleagues could be alleged here as an example. In order to demonstrate the potency of Pip as priming compounds, the expression of $A L D 1$ and PR1 was monitored upon infection of primed plants. As the authors present two independent experiments, it becomes apparent that the expression of both genes was twice as high in the wild type plants of one experiment than in the other. Even bigger fluctuations occurred, when Pseudomonas infection was used to investigate systemic immunity (Bernsdorff et al., 2016).

In order to study the NHP signaling without obstacles that infection studies might bring, we therefore decided to use UV-C treatment to induce the biosynthesis of Pip, respectively NHP (Article III). The idea originated from the previous reports showing that plants induce their SA biosynthesis upon treatment with ozone or UV-C light (Yalpani et al., 1994). Secondly, according to the expression database Arabidopsis eFP Browser (Winter et al., 2007), the expression of $A L D 1, S A R D 4$, and FMO1 is induced upon UV stress in Arabidopsis wild type plants. Consequently, when metabolic alterations were monitored by metabolite finger print analysis, the enhanced accumulation of Pip, NHP and NHP-OGlc could be detected already 8 hours after the UV-C treatment. This onset of accumulation corresponds to the time window in Arabidopsis leaves that were challenged by Pseudomonas infection (Hartmann et al., 2018). As it was shown that external SA could induce Pip biosynthesis (Navarova et al., 2012), it was necessary to exclude that UV-C triggered SA accumulation induces the NHP biosynthesis. Therefore, the formation of Pip and its metabolites was studied in the SA deficient mutants eds5 and pbs3. As previously shown in the SA biosynthesis mutant sid2, the formation of neither Pip nor NHP is affected by the absence of SA (Supplementary Figure2 (Hartmann et al., 2018)). Consequently, also the mutation in the PBS3 gene had no influence on the formation of Pip and its metabolites. On the other hand, however, it was not possible to detect any accumulation of these compounds in the eds5 mutant plants. Based on this observation, it was proposed that EDS5 could be responsible for the export of Pip out of the chloroplast. To tackle the question, whether Pip or NHP is transported by EDS5, the accumulation of Pip, NHP and NHP-OGlc in fmo1 plants upon UV-C stress was analyzed. As already seen in infection studies, only Pip accumulates in fmo1 plants, but neither NHP nor its glycoside (Chen et 
al., 2018). If EDS5 was an NHP exporter, Pip would accumulate upon UV-C treatment in eds5 as it was observed for fmo1 plants. Due to the absence of this Pip accumulation, it was concluded that EDS5 is most likely the exporter of Pip and not of NHP. The absence of Pip in the eds 5 mutant plants contradicts the general hypothesis of this thesis, as it was expected that the cargo of a transporter would accumulate in a similar fashion as the substrate of an enzyme upon mutation in the corresponding gene. However, the accumulation of metabolites in plastids seems to be tightly regulated. It is conceivable that the over-accumulation of a single compound might interfere with essential metabolic pathways within the plastids. Comparable, silencing of the phenylalanine exporter in Petunia hybrida cv Mitchell flowers led to a reduction of phenylalanine and its plastidial precursors arogenate and prephenate, instead of an accumulation of the cargo phenylalanine (Widhalm et al., 2015). In eds5, not only Pip is absent but also ISC, the precursor of ISC-9-Glu and consequently SA. Although it was demonstrated convincingly in Article II that EDS5 exports ISC in wild type plants, no accumulation of ISC could be detected in UV-C treated eds5 plants. In turn, an accumulation of ISC can be observed in the pbs3 mutant plants upon UV-C treatment, confirming the results from the autoimmunity mutant plants of Article II. Therefore, it seems likely that the expected accumulation of cargo upon mutation of the plastidial exporter EDS5 is channeled into other metabolic pathways and thus cannot be detected on a direct way.

To exclude unexpected pleiotropic effects of UV-C treatment, which could potentially cause the observed results, the accumulation of Pip and its metabolites was studied upon exogenous application of SA or Pip. To monitor the effects of external SA, metabolic alternations were examined in wild type, eds5 and pbs3 mutant plants 24 hours after SA soil drenching. In wild type plants, the SA treatment led, as expected (Navarova et al., 2012), to a production of Pip. A similar Pip accumulation trend, albeit less prominent, was observed in the pbs3 mutant. A comparable reduction was also previously reported (Navarova et al., 2012; Bernsdorff et al., 2016). It could be speculated that on the regulatory level, SA and Pip/NHP might function complementary. Therefore, a defect in the biosynthesis of one of these signaling compounds is sufficient to reduce the synthesis of the other. Consequently, the SA and Pip deficient double mutant sid2 ald1 shows a significantly higher susceptibility towards Pseudomonas infection (Bernsdorff et al., 2016). On the other hand, the eds5 mutant plants did not accumulate any Pip upon SA application, as already observed upon UV-C treatment. The inability to accumulate Pip in the eds5 mutants is therefore independent of SA, but most likely due to the direct 
involvement of EDS5 in the biosynthesis of NHP. Interestingly, neither NHP nor NHP-OGlc could be detected in none of the examined plants upon SA supplementation. One possible reason might be that the time point of the analysis was too early. SA, taken up after soil drench might need more time to reach a sufficient concentration in leaves to induce the biosynthesis of NHP. As depicted in the time course of Pip and its metabolites, Pip accumulation timely precedes the formation of NHP and its glycoside. Therefore, it seems conceivable that a later time point of analysis may enable the detection of NHP and NHPOGlc.

In order to monitor the general ability of eds5 mutant plants to synthesize NHP respectively NHP-OGlc, plants were soil drenched with Pip. 24 hours after soil application, eds5 plants accumulate only one third of NHP-OGlc in comparison to wild type plants. In the Pip biosynthesis mutant sard4, similar amounts of NHP-OGlc could be detected as in the eds 5 plants. As eds 5 and sard 4 mutant plants were in general able to synthesize NHPOGlc from external Pip, it was concluded that the reduced accumulation is due to the inability to synthesize Pip de novo in these mutants and therefore only Pip from the external source could be metabolized.

In summary, the results reported in Article III provide strong evidences for the involvement of EDS5 in the transport of Pip and therefore, in the biosynthesis of NHP. Possible other explanations for the observed results might be excluded by additional experiments. Surely, more direct evidences, like export assays with heterologous expressed EDS5, would consolidate the data presented so far. A challenge in this approach might be the establishment of a proper test system to examine the ability of EDS5 to transport Pip across a membrane. In their report from 2013, Serrano and colleagues tried to set up two of such test systems, one with protoplasts and a second with heterologous expression of EDS5 in yeast. Difficulties with rapid efflux of the loaded substances as well as endogenous yeast exporter(s) made it very difficult to gather convincing data (Serrano et al., 2013). Moreover, for those experiments ${ }^{14} \mathrm{C}$-labeled SA was utilized in order to distinguish endogenous and internal compounds. ${ }^{13} \mathrm{C}$ or ${ }^{14} \mathrm{C}$-labeled Pip could also be used to verify the conclusions from the soil drench experiment. Using such an approach, it could be monitored whether indeed only the external Pip is converted into NHP-OGlc in eds5 plants.

Interestingly, the biosynthesis of SA and NHP show a very similar pattern, if NHP is accepted as the active compound of Pip/NHP signaling. This assumption can be made based on the two independent reports from 2018. In Hartmann et al. 2018, NHP appears 
to be a more potent regulator of plant immunity than Pip (Hartmann et al., 2018). In the later report from Chen and colleagues, Pip itself only had a minimal influence on the immune response of Arabidopsis plants against an infection with a virulent strain of Pseudomonas. Application of NHP, on the other hand, led to an enhanced resistance against the same pathogen (Chen et al., 2018). Consequently, this strongly suggests that the precursors of the two main regulators of plant immunity, SA and NHP, are both synthesized in plastids. Prior to activation, ISC and Pip have to cross the plastidial membrane in order to be converted in the active substance. This spatial separation might be necessary for a proper regulation without the risk of premature activation of the plant immune system. Strikingly, both of these biosynthesis routes appear to use the same plastidial exporter, namely EDS5. This might explain why EDS5 expression is not affected in sid2 mutant and in NahG expressing plants (Nawrath, 2002). Keeping the importance of these signaling compounds for the plant immunity in mind, it appears reckless to channel both pathways through the same transporter. On the other hand, it seems even more surprising that this weak point of plant immunity is not exploited by pathogens or at least no effector was reported so far to have a direct interaction with EDS5. However, it has not been studied so far, whether those actions of HopI1 have any influence on EDS5 (Jelenska et al., 2007). HopI1 was shown to be active in the chloroplasts of infected plants. The expression of this effector leads to a reduced pathogen induced accumulation of SA. HopI1 was shown to interact with the heat shock protein Hsp70 and mediates a structural change in the thylakoids of affected cells. However, whether those actions of HopI 1 have any influence on EDS5 was not investigated so far. Taken together, the model presented in Article III strongly suggests that EDS5 is a major hub of the plant immunity and therefore it can be expected that further studies might decipher how plants can protect the integrity of EDS5 against pathogen-derived effectors.

In addition to the spatial distribution, also glycosylation seems to be a common feature of SA and NHP homeostasis. For SA, UGT74F2 was shown to be responsible for the pathogen induced formation of SAG (Song, 2006). Interestingly, a mutation in this gene enhances the resistance against Pseudomonas infections (Boachon et al., 2014), whereas the overexpression lines are more susceptible (Song et al., 2008). A similar pattern can be expected for an UGT, which can catalyze the conversion of NHP into NHP-OGlc. Since the knockout of this UGT would lead to an enhanced resistance, it is not surprising that previous mutant screens, which mostly focus on detecting mutants that are more 
susceptible (Jing et al., 2011), did not identify such an UGT yet. The findings elaborated in the sections above are integrated into the current model and depicted in Figure 7.

Several reports are proposing that there are two branches of plant immunity against biotrophic pathogens, of which one is SA dependent and the other SA independent (Bernsdorff et al., 2016). Recently, the Kachroo group showed that Pip acts upstream of

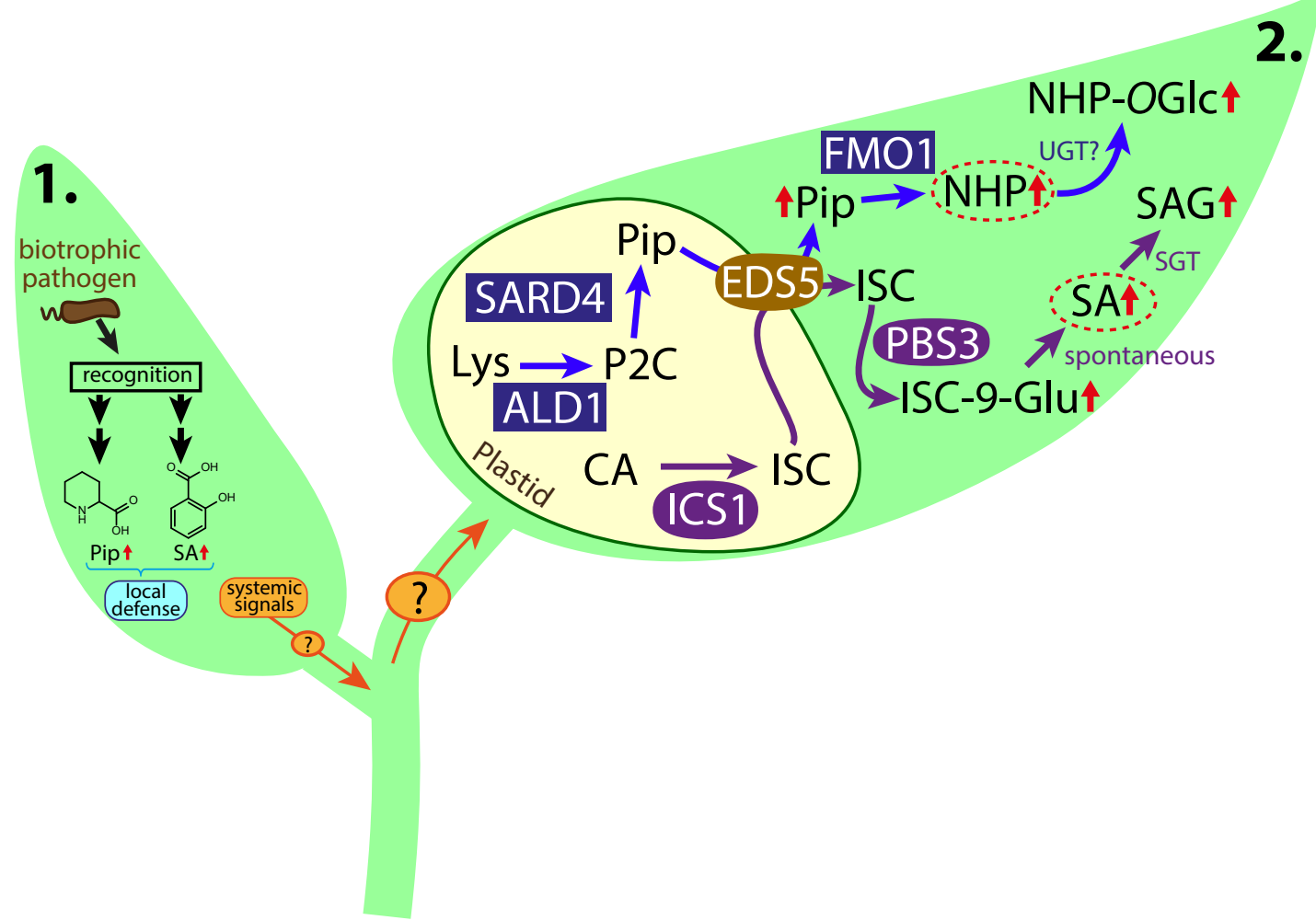

Figure 7. Current model for the induction of systemic acquired resistance (SAR) by biotrophic pathogens.

The recognition of biotrophic pathogens activates central regulators of the plant immunity. This stimulates, along other metabolic pathways, the biosynthesis of the signaling compounds SA and Pip/NHP. These signaling compounds orchestrate the local defense against the invader. In addition to the basal response, chemical signals are generated, which spread throughout the plant. In distal tissues, these signals induce changes in histone modifications, gene expression and metabolic alterations. Once again, the biosynthesis of Pip/NHP and SA is activated. Both biosynthesis pathways (blue for NHP, purple for SA) originate in plastids. The $\varepsilon$-amino transferase ALD1 catalyzes the conversion from lysine into P2C, which is subsequently reduced into Pip by SARD4. Pip is exported into the cytosol by EDS5, where it is N-hydroxylated by FMO1 to NHP. A yet unknown UGT glycosylates NHP into NHP-OGlc. For the SA biosynthesis, ICS1 catalyzes the isomerization of CA into ISC in plastids. ISC is subsequently transported into the cytosol, where it is conjugated with Glu to yield ISC-9-Glu by PBS3. ISC-9-Glu decays non-enzymatically and thereby releases SA. Finally, SA is glycosylated by SGT to SAG. Red arrow represents the metabolic accumulation. The signaling compounds SA and NHP are marked with a dashed red circle.

Abbreviations: chorismate (CA), isochorismate (ISC), isochorismate-9-glutamate (ISC-9-Glu), salicylic acid (SA), SA glycoside (SAG), lysine (Lys), $\Delta^{1}$-piperideine-2-carboxylic acid (P2C), pipecolic acid (Pip), $\mathrm{N}$-hydroxy pipecolic acid (NHP), NHP glycoside (NHP-OGlc), AGD2-like defense response protein 1 (ALD1), systemic acquired resistance-deficient 4 (SARD4), isochorismate synthase 1 (ICS1), enhanced disease susceptibility 5 (EDS5), flavin-dependent monooxygenase 1 (FMO1), avrPphB susceptible 3 (PBS3), SA glycosyltransferase (SGT), UDP-dependent glycosyltransferase (UGT). 
Aza respectively of G3P (Wang et al., 2018). The signaling pathway of Pip, NHP, Aza and G3P might indeed work independently of SA and therefore represent the previously proposed second branch. From the results presented in Article III it can be deduced that there are some intersections between the SA and the NHP pathways.

As it was shown that UV-C treatment could activate the NHP biosynthesis in a SAindependent manner, this method might be instrumental in the future to study this branch of plant immunity. It remains to be determined if the other proposed components of this signaling pathway (Aza and G3P) accumulate upon UV-C treatment in addition to NHP. For the SA-independent branch, all four metabolites Pip, NHP, Aza and G3P were proposed as mobile signals (Navarova et al., 2012; Chen et al., 2018; Wang et al., 2018). It is unlikely though that all of these compounds are transported to distal tissues in order to induce SAR (Wang et al., 2018). Precise dissection will be required to distinguish, which of these metabolites are necessary for the translocation and for which de novo synthesis in systemic organs might be sufficient to establish SAR.

\subsection{Concluding remarks}

In the last twenty years, the understanding of plant immunity has constantly improved. One major advance was the complete sequencing of the Arabidopsis genome (The Arabidopsis Genome Initiative, 2000). Despite all the progress made ever since, the understanding of such a complex interplay of gene regulations, signaling cascades, spatialtemporary distribution and metabolic pathways, as found in plant immunity, is still fragmented. The work presented in this thesis may add some so far missing parts to the overall picture.

Several conclusions can be drawn from the results presented herein. The continuous improvements achieved in the development of high-resolution mass spectrometry now allow to find new components in so far not fully understood pathways. Tandem fragmentation mass spectrometry and computational analysis of these data will improve and therefore enable the unambiguous identification of even additional metabolic markers. An example is the identification and the unequivocally structural characterization of ISC-9-Glu as a crucial metabolite in the SA-pathway. In the initial metabolite fingerprint analysis, this feature was detected, but due to the incomplete metabolome annotation and the limited number of MS/MS spectra in online data bases, this feature would have been dismissed without any additional information. The 
identification, however, enabled the reconstruction of a crucial metabolic pathway, which has been studied for several decades. It was possible to assign the role of PBS3 to this pathway. This gene was genetically connected to the SA biosynthesis pathway, but its precise function was unknown. The presence of an appropriate knockout mutant respectively a collection of autoimmunity mutants was essential for this discovery. The combination of biochemical analytic and genetics-driven mutant screens will presumably be crucial to further elucidate plant immunity. Therefore, it is important to challenge seemingly fixed concepts. For example, the results, which led to the conclusion that SA is synthesized completely in plastids, could be integrated into the current model, although they seemed contradictory at first sight. For EDS5 the exclusive connection to SA was rarely questioned, although its gene expression could be observed in SA deficient mutants (Nawrath, 2002). The probable involvement of EDS5 in the NHP biosynthesis shows, however, an important connection between both biosynthesis pathways. Supportive evidences, like export assays with heterologously expressed EDS5, would consolidate the data presented so far. The next potential question to tackle, however, should be the identification of a cytosolic receptor for NHP. Due to the structural similarities between SA and NHP (Hartmann and Zeier, 2018), it might be reasonable to test the capacity of NPR1/3/4 to bind NHP. Interestingly, npr1 mutant plants seem to accumulate more NHP upon infection than wild type plants (Hartmann et al., 2018), which is reminiscent of the enhanced SA accumulation in the same mutant plants (Delaney et al., 1995).

SA and NHP seem to be key regulators of plant immunity within leaves. However, the mobile signal that spreads from infected leaves towards distal tissues is still elusive. As the number of tentative signaling compounds grows continuously, the time may have come to change the assumption of a single metabolite that induces SAR. Another concept could be that the phloem content is monitored constantly. Upon infection, infested organs reorganize their metabolism (Bolton, 2009). Thereby, a relatively large number of metabolites is exudated and consequently changes the phloem composition. Upon reaching particular thresholds, distal tissues could recognize that this change is due to a pathogenic attack and therefore, initiate the reprogramming, which consequently leads to the establishment of SAR. This concept would explain why the number of identified signals is growing continuously, and why the external addition of individual compounds not always leads to an induction of SAR (Shah and Zeier, 2013). Additionally, this model would incorporate the immunity related activity of primary metabolites such as citrate (Finkemeier et al., 2013), fumarate (Balmer et al., 2018), malate (Zhao et al., 2018) and 
different sugars (Bolouri Moghaddam and Van den Ende, 2012). Several mutant lines, including sard4, are reported to have a robust local defense against Pseudomonas infections, but fail to establish SAR. A study of the metabolites present in their petiole exudates might show high similarities to the exudates of infected wild type plants. This comparison could help to identify the exudate metabolome.

In summary, great progress was achieved in the understanding of plant immunity during the last decade. However, due to the continuously improving detection techniques, the number of open questions grows equally with every new finding. Consequently, the work presented here could solve some longstanding unanswered questions and at the same time raised new issues for subsequent research. 


\section{References}

Adam, A.L., Nagy, Z.A., Katay, G., Mergenthaler, E., and Viczian, 0. (2018). Signals of systemic immunity in plants: progress and open questions. International Journal of Molecular Sciences 19, 1146.

Attaran, E., Zeier, T.E., Griebel, T., and Zeier, J. (2009). Methyl salicylate production and jasmonate signaling are not essential for systemic acquired resistance in Arabidopsis. The Plant Cell 21, 954-971.

Bacete, L., Melida, H., Miedes, E., and Molina, A. (2018). Plant cell wall-mediated immunity: cell wall changes trigger disease resistance responses. The Plant Journal 93, 614-636.

Balmer, A., Pastor, V., Glauser, G., and Mauch-Mani, B. (2018). Tricarboxylates induce defense priming against bacteria in Arabidopsis thaliana. Frontiers in Plant Science 9, 1221.

Balmer, A., Pastor, V., Gamir, J., Flors, V., and Mauch-Mani, B. (2015). The 'prime-ome': towards a holistic approach to priming. Trends in Plant Science 20, 443-452.

Beckers, G.J., Jaskiewicz, M., Liu, Y., Underwood, W.R., He, S.Y., Zhang, S., and Conrath, U. (2009). Mitogen-activated protein kinases 3 and 6 are required for full priming of stress responses in Arabidopsis thaliana. The Plant Cell 21, 944-953.

Berens, M.L., Wolinska, K.W., Spaepen, S., Ziegler, J., Nobori, T., Nair, A., Kruler, V., Winkelmuller, T.M., Wang, Y., Mine, A., Becker, D., Garrido-Oter, R., SchulzeLefert, P., and Tsuda, K. (2019). Balancing trade-offs between biotic and abiotic stress responses through leaf age-dependent variation in stress hormone crosstalk. Proceedings of the National Academy of Sciences of the United States of America 116, 2364-2373.

Bernsdorff, F. (2014). "On the role of amino acids in plant disease resistance: Interplay between pipecolic acid and salicylic acid in plant systemic acquired resistance", thesis. Heinrich Heine University Duesseldorf.

Bernsdorff, F., Doring, A.C., Gruner, K., Schuck, S., Brautigam, A., and Zeier, J. (2016). Pipecolic acid orchestrates plant systemic acquired resistance and defense priming via salicylic acid-dependent and -independent pathways. The Plant Cell 28, 102-129.

Betsuyaku, S., Katou, S., Takebayashi, Y., Sakakibara, H., Nomura, N., and Fukuda, H. (2018). Salicylic acid and jasmonic acid pathways are activated in spatially different domains around the infection site during effector-triggered immunity in Arabidopsis thaliana. Plant and Cell Physiology 59, 8-16.

Bigeard, J., Colcombet, J., and Hirt, H. (2015). Signaling mechanisms in patterntriggered immunity (PTI). Molecular Plant 8, 521-539.

Boachon, B., Gamir, J., Pastor, V., Erb, M., Dean, J.V., Flors, V., and Mauch-Mani, B. (2014). Role of two UDP-glycosyltransferases from the L group of arabidopsis in resistance against pseudomonas syringae. European Journal of Plant Pathology 139, 707-720.

Boiteau, R., Hoyt, D., Nicora, C., Kinmonth-Schultz, H., Ward, J., and Bingol, K. (2018). Structure elucidation of unknown metabolites in metabolomics by combined NMR and MS/MS prediction. Metabolites 8, 8 . 
Bolouri Moghaddam, M.R., and Van den Ende, W. (2012). Sugars and plant innate immunity. Journal of Experimental Botany 63, 3989-3998.

Bolton, M.D. (2009). Primary metabolism and plant defense - fuel for the fire. Molecular Plant-Microbe Interactions 22, 487-497.

Boutrot, F., and Zipfel, C. (2017). Function, discovery, and exploitation of plant pattern recognition receptors for broad-spectrum disease resistance. Annual Review of Phytopathology 55, 257-286.

Buchner, A. (1828). Ueber das Rigatellische Fiebermittel und über eine in der Weidenrinde entdeckte alkaloidische Substanz. Repertorium für die Pharmacie 29, 405-420.

Caspi, R., Altman, T., Dreher, K., Fulcher, C.A., Subhraveti, P., Keseler, I.M., Kothari, A., Krummenacker, M., Latendresse, M., Mueller, L.A., Ong, Q., Paley, S., Pujar, A., Shearer, A.G., Travers, M., Weerasinghe, D., Zhang, P., and Karp, P.D. (2012). The MetaCyc database of metabolic pathways and enzymes and the BioCyc collection of pathway/genome databases. Nucleic Acids Research 40, D742-753.

Chanda, B., Xia, Y., Mandal, M.K., Yu, K., Sekine, K.T., Gao, Q.M., Selote, D., Hu, Y., Stromberg, A., Navarre, D., Kachroo, A., and Kachroo, P. (2011). Glycerol-3phosphate is a critical mobile inducer of systemic immunity in plants. Nature Genetics 43, 421-427.

Chang, M., Zhao, J., Chen, H., Li, G., Chen, J., Li, M., Palmer, I.A., Song, J., Alfano, J.R., Liu, F., and Fu, Z.Q. (2019). PBS3 protects EDS1 from proteasome-mediated degradation in plant immunity. Molecular Plant, doi:10.1016/.molp.2019.01.023.

Chaturvedi, R., Venables, B., Petros, R.A., Nalam, V., Li, M., Wang, X., Takemoto, L.J., and Shah, J. (2012). An abietane diterpenoid is a potent activator of systemic acquired resistance. The Plant Journal 71, 161-172.

Chen, Q., Westfall, C.S., Hicks, L.M., Wang, S., and Jez, J.M. (2010). Kinetic basis for the conjugation of auxin by a GH3 family indole-acetic acid-amido synthetase. Journal of Biological Chemistry 285, 29780-29786.

Chen, Y.C., Holmes, E.C., Rajniak, J., Kim, J.G., Tang, S., Fischer, C.R., Mudgett, M.B., and Sattely, E.S. (2018). $N$-hydroxy-pipecolic acid is a mobile metabolite that induces systemic disease resistance in Arabidopsis. Proceedings of the National Academy of Sciences of the United States of America 115, E4920-E4929.

Chester, K.S. (1933). The problem of acquired physiological immunity in plants. The Quarterly Review of Biology 8, 275-324.

Coll, N.S., Epple, P., and Dangl, J.L. (2011). Programmed cell death in the plant immune system. Cell Death \& Differentiation 18, 1247-1256.

Collier, S.M., and Moffett, P. (2009). NB-LRRs work a "bait and switch" on pathogens. Trends in Plant Science 14, 521-529.

Cook, D.E., Mesarich, C.H., and Thomma, B.P. (2015). Understanding plant immunity as a surveillance system to detect invasion. Annual Review of Phytopathology 53, 541-563.

Coquoz, J.-L., Buchala, A., and Métraux, J.-P. (1998). The biosynthesis of salicylic acid in potato plants. Plant Physiology 117, 1095-1101.

Creydt, M., and Fischer, M. (2017). Plant metabolomics: maximizing metabolome coverage by optimizing mobile phase additives for nontargeted mass spectrometry in positive and negative electrospray ionization mode. Analytical Chemistry 89, 10474-10486.

da Silva, R.R., Dorrestein, P.C., and Quinn, R.A. (2015). Illuminating the dark matter in metabolomics. Proceedings of the National Academy of Sciences of the United States of America 112, 12549-12550. 
Dean, J.V., Mohammed, L.A., and Fitzpatrick, T. (2005). The formation, vacuolar localization, and tonoplast transport of salicylic acid glucose conjugates in tobacco cell suspension cultures. Planta 221, 287-296.

DeClue, M.S., Baldridge, K.K., Kast, P., and Hilvert, D. (2006). Experimental and computational investigation of the uncatalyzed rearrangement and elimination reactions of isochorismate. Journal of the American Chemical Society 128, 20432051.

Delaney, T., Friedrich, L., and Ryals, J. (1995). Arabidopsis signal transduction mutant defective in chemically and biologically induced disease resistance. Proceedings of the National Academy of Sciences of the United States of America 92, 6602-6606.

Dempsey, D.A., Vlot, A.C., Wildermuth, M.C., and Klessig, D.F. (2011). Salicylic acid biosynthesis and metabolism. Arabidopsis Book 9, e0156.

Ding, Y., Sun, T., Ao, K., Peng, Y., Zhang, Y., Li, X., and Zhang, Y. (2018). Opposite roles of salicylic acid receptors NPR1 and NPR3/NPR4 in transcriptional regulation of plant immunity. Cell 173, 1454-1467 e1415.

Djamei, A., Schipper, K., Rabe, F., Ghosh, A., Vincon, V., Kahnt, J., Osorio, S., Tohge, T., Fernie, A.R., Feussner, I., Feussner, K., Meinicke, P., Stierhof, Y.D., Schwarz, H., Macek, B., Mann, M., and Kahmann, R. (2011). Metabolic priming by a secreted fungal effector. Nature 478, 395-398.

El-Basyouni, S.Z., Chen, D., Ibrahim, R., Neish, A., and Towers, G. (1964). The biosynthesis of hydroxybenzoic acids in higher plants. Phytochemistry 3, 485-492.

Emwas, A.H. (2015). The strengths and weaknesses of NMR spectroscopy and mass spectrometry with particular focus on metabolomics research. Methods in Molecular Biology 1277, 161-193.

Etalo, D.W., De Vos, R.C., Joosten, M.H., and Hall, R.D. (2015). Spatially resolved plant metabolomics: some potentials and limitations of laser-ablation electrospray ionization mass spectrometry metabolite imaging. Plant Physiology 169, 14241435.

Feussner, I., and Polle, A. (2015). What the transcriptome does not tell - proteomics and metabolomics are closer to the plants' patho-phenotype. Current Opinion in Plant Biology 26, 26-31.

Finkelstein, R. (2013). Abscisic acid synthesis and response. The Arabidopsis book/American Society of Plant Biologists 11.

Finkemeier, I., Konig, A.C., Heard, W., Nunes-Nesi, A., Pham, P.A., Leister, D., Fernie, A.R., and Sweetlove, L.J. (2013). Transcriptomic analysis of the role of carboxylic acids in metabolite signaling in Arabidopsis leaves. Plant Physiology 162, 239-253.

Finnegan, T., Steenkamp, P.A., Piater, L.A., and Dubery, I.A. (2016). The lipopolysaccharide-induced metabolome signature in Arabidopsis thaliana reveals dynamic reprogramming of phytoalexin and phytoanticipin pathways. PLoS One 11, e0163572.

Flor, H.H. (1971). Current status of the gene-for-gene concept. Annual Review of Phytopathology 9, 275-296.

Fragniere, C., Serrano, M., Abou-Mansour, E., Metraux, J.P., and L'Haridon, F. (2011). Salicylic acid and its location in response to biotic and abiotic stress. FEBS Letters 585, 1847-1852.

Franceschini, S., Fedkenheuer, M., Vogelaar, N.J., Robinson, H.H., Sobrado, P., and Mattevi, A. (2012). Structural insight into the mechanism of oxygen activation and substrate selectivity of flavin-dependent N-hydroxylating monooxygenases. Biochemistry 51, 7043-7045. 
Fu, Z.Q., and Dong, X. (2013). Systemic acquired resistance: turning local infection into global defense. Annual Review of Plant Biology 64, 839-863.

Gaffney, T., Friedrich, L., Vernooij, B., Negrotto, D., Nye, G., Uknes, S., Ward, E., Kessmann, H., and Ryals, J. (1993). Requirement of salicylic acid for the induction of systemic acquired resistance. Science 261, 754-756.

Galeano Garcia, P., Neves Dos Santos, F., Zanotta, S., Eberlin, M.N., and Carazzone, C. (2018). Metabolomics of solanum lycopersicum infected with Phytophthora infestans leads to early detection of late blight in asymptomatic plants. Molecules 23, 3330.

Galili, G., Tang, G., Zhu, X., and Gakiere, B. (2001). Lysine catabolism: a stress and development super-regulated metabolic pathway. Current Opinion in Plant Biology 4, 261-266.

Garcion, C., Lohmann, A., Lamodiere, E., Catinot, J., Buchala, A., Doermann, P., and Metraux, J.P. (2008). Characterization and biological function of the ISOCHORISMATE SYNTHASE 2 gene of Arabidopsis. Plant Physiology 147, 12791287.

Gilroy, S., Bialasek, M., Suzuki, N., Gorecka, M., Devireddy, A.R., Karpinski, S., and Mittler, R. (2016). ROS, calcium, and electric signals: key mediators of rapid systemic signaling in plants. Plant Physiology 171, 1606-1615.

Glazebrook, J., Rogers, E.E., and Ausubel, F.M. (1996). Isolation of Arabidopsis mutants with enhanced disease susceptibility by direct screening. Genetics 143, 973-982.

Gonçalves-Butruille, M., Szajner, P., Torigoi, E., Leite, A., and Arruda, P. (1996). Purification and characterization of the bifunctional enzyme lysine-ketoglutarate reductase-saccharopine dehydrogenase from maize. Plant Physiology 110, 765771.

Goyer, A., Johnson, T.L., Olsen, L.J., Collakova, E., Shachar-Hill, Y., Rhodes, D., and Hanson, A.D. (2004). Characterization and metabolic function of a peroxisomal sarcosine and pipecolate oxidase from Arabidopsis. Journal of Biological Chemistry 279, 16947-16953.

Gross, J., Cho, W.K., Lezhneva, L., Falk, J., Krupinska, K., Shinozaki, K., Seki, M., Herrmann, R.G., and Meurer, J. (2006). A plant locus essential for phylloquinone (vitamin K1) biosynthesis originated from a fusion of four eubacterial genes. Journal of Biological Chemistry 281, 17189-17196.

Gruner, K., Griebel, T., Navarova, H., Attaran, E., and Zeier, J. (2013). Reprogramming of plants during systemic acquired resistance. Frontiers in Plant Science 4, 252.

Guijas, C., Montenegro-Burke, J.R., Domingo-Almenara, X., Palermo, A., Warth, B., Hermann, G., Koellensperger, G., Huan, T., Uritboonthai, W., Aisporna, A.E., Wolan, D.W., Spilker, M.E., Benton, H.P., and Siuzdak, G. (2018). METLIN: a technology platform for identifying knowns and unknowns. Analytical Chemistry 90, 3156-3164.

Gupta, R.N., and Spenser, I.D. (1969). Biosynthesis of the piperidine nucleus: THE MODE OF INCORPORATION OF LYSINE INTO PIPECOLIC ACID AND INTO PIPERIDINE ALKALOIDS. Journal of Biological Chemistry 244, 88-94.

Hagen, G., Kleinschmidt, A., and Guilfoyle, T. (1984). Auxin-regulated gene expression in intact soybean hypocotyl and excised hypocotyl sections. Planta 162, 147-153.

Hallen, A., Cooper, A.J., Jamie, J.F., and Karuso, P. (2015). Insights into enzyme catalysis and thyroid hormone regulation of cerebral ketimine reductase/ $\mu$-crystallin under physiological conditions. Neurochemical Research 40,1252-1266.

Hamberg, M., and Gardner, H.W. (1992). Oxylipin pathway to jasmonates: biochemistry and biological significance. Biochimica et Biophysica Acta 1165, 1-18. 
Hansen, B.G., Kliebenstein, D.J., and Halkier, B.A. (2007). Identification of a flavinmonooxygenase as the S-oxygenating enzyme in aliphatic glucosinolate biosynthesis in Arabidopsis. The Plant Journal 50, 902-910.

Haroth, S. (2018). "Metabolic fate of jasmonates", thesis. University of Goettingen.

Hartmann, M., and Zeier, J. (2018). L-lysine metabolism to $N$-hydroxypipecolic acid: an integral immune-activating pathway in plants. The Plant Journal 96, 5-21.

Hartmann, M., Kim, D., Bernsdorff, F., Ajami-Rashidi, Z., Scholten, N., Schreiber, S., Zeier, T., Schuck, S., Reichel-Deland, V., and Zeier, J. (2017). Biochemical principles and functional aspects of pipecolic acid biosynthesis in plant immunity. Plant Physiology 174, 124-153.

Hartmann, M., Zeier, T., Bernsdorff, F., Reichel-Deland, V., Kim, D., Hohmann, M., Scholten, N., Schuck, S., Brautigam, A., Holzel, T., Ganter, C., and Zeier, J. (2018). Flavin monooxygenase-generated N-hydroxypipecolic acid is a critical element of plant systemic immunity. Cell 173, 456-469 e416.

Hayat, S., Hayat, Q., Alyemeni, M.N., Wani, A.S., Pichtel, J., and Ahmad, A. (2012). Role of proline under changing environments: a review. Plant Signaling \& Behavior 7, 1456-1466.

Heck, S., Grau, T., Buchala, A., Métraux, J.P., and Nawrath, C. (2003). Genetic evidence that expression of $N a h G$ modifies defence pathways independent of salicylic acid biosynthesis in the Arabidopsis-Pseudomonas syringae pv. tomato interaction. The Plant Journal 36, 342-352.

Higashino, K., Fujioka, M., and Yamamura, Y. (1971). The conversion of L-lysine to saccharopine and $\alpha$-aminoadipate in mouse. Archives of Biochemistry and Biophysics 142, 606-614.

Holmes, E., Chen, Y.C., Sattely, E., and Mudgett, M.B. (2019). Conservation of $N$ hydroxy-pipecolic acid-mediated systemic acquired resistance in crop plants. bioRxiv, 537597.

Horai, H., Arita, M., Kanaya, S., Nihei, Y., Ikeda, T., Suwa, K., Ojima, Y., Tanaka, K., Tanaka, S., Aoshima, K., Oda, Y., Kakazu, Y., Kusano, M., Tohge, T., Matsuda, F., Sawada, Y., Hirai, M.Y., Nakanishi, H., Ikeda, K., Akimoto, N., Maoka, T., Takahashi, H., Ara, T., Sakurai, N., Suzuki, H., Shibata, D., Neumann, S., Iida, T., Tanaka, K., Funatsu, K., Matsuura, F., Soga, T., Taguchi, R., Saito, K., and Nishioka, T. (2010). MassBank: a public repository for sharing mass spectral data for life sciences. Journal of Mass Spectrometry 45, 703-714.

Huang, J., Gu, M., Lai, Z., Fan, B., Shi, K., Zhou, Y.H., Yu, J.Q., and Chen, Z. (2010). Functional analysis of the Arabidopsis PAL gene family in plant growth, development, and response to environmental stress. Plant Physiology 153, 15261538.

Hunter, L.J., Westwood, J.H., Heath, G., Macaulay, K., Smith, A.G., Macfarlane, S.A., Palukaitis, P., and Carr, J.P. (2013). Regulation of RNA-dependent RNA polymerase 1 and isochorismate synthase gene expression in Arabidopsis. PLoS One 8, e66530.

Jagadeeswaran, G., Raina, S., Acharya, B.R., Maqbool, S.B., Mosher, S.L., Appel, H.M., Schultz, J.C., Klessig, D.F., and Raina, R. (2007). Arabidopsis GH3-LIKE DEFENSE GENE 1 is required for accumulation of salicylic acid, activation of defense responses and resistance to Pseudomonas syringae. The Plant Journal 51, 234-246.

Jelenska, J., Yao, N., Vinatzer, B.A., Wright, C.M., Brodsky, J.L., and Greenberg, J.T. (2007). A J domain virulence effector of Pseudomonas syringae remodels host chloroplasts and suppresses defenses. Current Biology 17, 499-508. 
Jenns, A., and Kuc, J. (1979). Graft transmission of systemic resistance of cucumber to anthracnose induced by Colletotrichum lagenarium and tobacco necrosis virus. Phytopathology 69, 753-756..

Jing, B., Xu, S., Xu, M., Li, Y., Li, S., Ding, J., and Zhang, Y. (2011). Brush and spray: a highthroughput systemic acquired resistance assay suitable for large-scale genetic screening. Plant Physiology 157, 973-980.

Johansen, V.E., Catón, L., Hamidjaja, R., Oosterink, E., Wilts, B.D., Rasmussen, T.S., Sherlock, M.M., Ingham, C.J., and Vignolini, S. (2018). Genetic manipulation of structural color in bacterial colonies. Proceedings of the National Academy of Sciences of the United States of America 115, 2652-2657.

Jones, E.E., and Broquist, H.P. (1966). Saccharopine, an intermediate of the aminoadipic acid pathway of lysine biosynthesis III. AMINOADIPIC SEMIALDEHYDEGLUTAMATE REDUCTASE. Journal of Biological Chemistry 241, 3430-3434.

Jones, J.D., and Dangl, J.L. (2006). The plant immune system. Nature 444, 323-329.

Jung, G.Y., Park, J.Y., Choi, H.J., Yoo, S.J., Park, J.K., and Jung, H.W. (2016). A rice gene homologous to Arabidopsis AGD2-LIKE DEFENSE 1 participates in disease resistance response against infection with Magnaporthe oryzae. The Plant Pathology Journal 32, 357-362.

Jung, H.W., Tschaplinski, T.J., Wang, L., Glazebrook, J., and Greenberg, J.T. (2009). Priming in systemic plant immunity. Science 324, 89-91.

Kachroo, A., and Robin, G.P. (2013). Systemic signaling during plant defense. Current Opinion in Plant Biology 16, 527-533.

Kaever, A., Landesfeind, M., Possienke, M., Feussner, K., Feussner, I., and Meinicke, P. (2012). MarVis-Filter: ranking, filtering, adduct and isotope correction of mass spectrometry data. Journal of Biomedicine and Biotechnology 2012, 263910.

Kaever, A., Landesfeind, M., Feussner, K., Mosblech, A., Heilmann, I., Morgenstern, B., Feussner, I., and Meinicke, P. (2015). MarVis-Pathway: integrative and exploratory pathway analysis of non-targeted metabolomics data. Metabolomics 11, 764-777.

Kanehisa, M., Goto, S., Sato, Y., Furumichi, M., and Tanabe, M. (2012). KEGG for integration and interpretation of large-scale molecular data sets. Nucleic Acids Research 40, D109-114.

Katsir, L., Schilmiller, A.L., Staswick, P.E., He, S.Y., and Howe, G.A. (2008). COI1 is a critical component of a receptor for jasmonate and the bacterial virulence factor coronatine. Proceedings of the National Academy of Sciences of the United States of America 105, 7100-7105.

Keller, M.A., Piedrafita, G., and Ralser, M. (2015). The widespread role of nonenzymatic reactions in cellular metabolism. Current Opinion in Biotechnology 34, 153-161.

Khan, M., Subramaniam, R., and Desveaux, D. (2016). Of guards, decoys, baits and traps: pathogen perception in plants by type III effector sensors. Current Opinion in Microbiology 29, 49-55.

Klämbt, H. (1962). Conversion in plants of benzoic acid to salicylic acid and its $\beta \mathrm{d}-$ glucoside. Nature 196, 491.

Klessig, D.F., Choi, H.W., and Dempsey, D.A. (2018). Systemic acquired resistance and salicylic acid: past, present, and future. Molecular Plant-Microbe Interactions 31, 871-888.

Koch, M., Vorwerk, S., Masur, C., Sharifi-Sirchi, G., Olivieri, N., and Schlaich, N.L. (2006). A role for a flavin-containing mono-oxygenase in resistance against microbial pathogens in Arabidopsis. The Plant Journal 47, 629-639. 
Kolbe, H. (1860). Ueber Synthese der Salicylsäure. Justus Liebigs Annalen der Chemie 113, 125-127.

König, S., Feussner, K., Kaever, A., Landesfeind, M., Thurow, C., Karlovsky, P., Gatz, C., Polle, A., and Feussner, I. (2014). Soluble phenylpropanoids are involved in the defense response of Arabidopsis against Verticillium longisporum. New Phytologist 202, 823-837.

Kriechbaumer, V., Botchway, S.W., and Hawes, C. (2016). Localization and interactions between Arabidopsis auxin biosynthetic enzymes in the TAA/YUC-dependent pathway. Journal of Experimental Botany 68, 4195-4207.

Lamb, A.L. (2015). Breaking a pathogen's iron will: Inhibiting siderophore production as an antimicrobial strategy. Biochimica et Biophysica Acta 1854, 1054-1070.

Lassowskat, I., Bottcher, C., Eschen-Lippold, L., Scheel, D., and Lee, J. (2014). Sustained mitogen-activated protein kinase activation reprograms defense metabolism and phosphoprotein profile in Arabidopsis thaliana. Frontiers in Plant Science 5, 554.

Li, J., Kristiansen, K.A., Hansen, B.G., and Halkier, B.A. (2010). Cellular and subcellular localization of flavin-monooxygenases involved in glucosinolate biosynthesis. Journal of Experimental Botany 62, 1337-1346.

Li, Y., Baldauf, S., Lim, E.K., and Bowles, D.J. (2001). Phylogenetic analysis of the UDPglycosyltransferase multigene family of Arabidopsis thaliana. Journal of Biological Chemistry 276, 4338-4343.

Lim, E.K., Doucet, C.J., Li, Y., Elias, L., Worrall, D., Spencer, S.P., Ross, J., and Bowles, D.J. (2002). The activity of Arabidopsis glycosyltransferases toward salicylic acid, 4-hydroxybenzoic acid, and other benzoates. Journal of Biological Chemistry 277, 586-592.

Liu, L., Sonbol, F.-M., Huot, B., Gu, Y., Withers, J., Mwimba, M., Yao, J., He, S.Y., and Dong, X. (2016). Salicylic acid receptors activate jasmonic acid signalling through a non-canonical pathway to promote effector-triggered immunity. Nature Communications 7, 13099.

Liu, P.P., von Dahl, C.C., and Klessig, D.F. (2011). The extent to which methyl salicylate is required for signaling systemic acquired resistance is dependent on exposure to light after infection. Plant Physiology 157, 2216-2226.

Liu, T., Song, T., Zhang, X., Yuan, H., Su, L., Li, W., Xu, J., Liu, S., Chen, L., Chen, T., Zhang, M., Gu, L., Zhang, B., and Dou, D. (2014). Unconventionally secreted effectors of two filamentous pathogens target plant salicylate biosynthesis. Nature Communications 5, 4686.

Lu, S.-P., and Lewin, A.H. (1998). Enamine/imine tautomerism in $\alpha, \beta$-unsaturated- $\alpha$ amino acids. Tetrahedron 54, 15097-15104.

Lu, Y., and Yao, J. (2018). Chloroplasts at the crossroad of photosynthesis, pathogen infection and plant defense. International Journal of Molecular Sciences 19, 3900.

Ludwig, M., Duhrkop, K., and Bocker, S. (2018). Bayesian networks for mass spectrometric metabolite identification via molecular fingerprints. Bioinformatics 34, i333-i340.

Luna, E., Bruce, T.J., Roberts, M.R., Flors, V., and Ton, J. (2012). Next-generation systemic acquired resistance. Plant Physiology 158, 844-853.

Luo, Q., Meneely, K.M., and Lamb, A.L. (2011). Entropic and enthalpic components of catalysis in the mutase and lyase activities of Pseudomonas aeruginosa PchB. Journal of the American Chemical Society 133, 7229-7233.

Maeda, H., and Dudareva, N. (2012). The shikimate pathway and aromatic amino acid biosynthesis in plants. Annual Review of Plant Biology 63, 73-105. 
Maeda, H., Yoo, H., and Dudareva, N. (2011). Prephenate aminotransferase directs plant phenylalanine biosynthesis via arogenate. Nature Chemical Biology 7, 19-21.

Malamy, J., Carr, J.P., Klessig, D.F., and Raskin, I. (1990). Salicylic acid: a likely endogenous signal in the resistance response of tobacco to viral infection. Science 250, 1002-1004.

Mann, P. (1955). Purification and properties of the amine oxidase of pea seedlings. Biochemical Journal 59, 609.

Marti, S., Andrés, J., Moliner, V., Silla, E., Tunon, I., and Bertrán, J. (2009). Mechanism and plasticity of isochorismate pyruvate lyase: a computational study. Journal of the American Chemical Society 131, 16156-16161.

Martinez-Medina, A., Flors, V., Heil, M., Mauch-Mani, B., Pieterse, C.M.J., Pozo, M.J., Ton, J., van Dam, N.M., and Conrath, U. (2016). Recognizing plant defense priming. Trends in Plant Science 21, 818-822.

Mauch-Mani, B., and Slusarenko, A.J. (1996). Production of salicylic acid precursors is a major function of phenylalanine ammonia-lyase in the resistance of Arabidopsis to Peronospora parasitica. The Plant Cell 8, 203-212.

Meeuse, B.J., and Raskin, I. (1988). Sexual reproduction in the arum lily family, with emphasis on thermogenicity. Sexual Plant Reproduction 1, 3-15.

Meister, A. (1954). The $\alpha$-keto analogues of arginine, ornithine, and lysine. Journal of Biological Chemistry 206, 577-585.

Meller, B., Kuznicki, D., Arasimowicz-Jelonek, M., Deckert, J., and FloryszakWieczorek, J. (2018). BABA-primed histone modifications in potato for intergenerational resistance to Phytophthora infestans. Frontiers in Plant Science 9, 1228.

Métraux, J., Signer, H., Ryals, J., Ward, E., Wyss-Benz, M., Gaudin, J., Raschdorf, K., Schmid, E., Blum, W., and Inverardi, B. (1990). Increase in salicylic acid the onset of systemic acquired resistance in cucumber. Science 250, 1004-1006.

Mishina, T.E., and Zeier, J. (2006). The Arabidopsis flavin-dependent monooxygenase FMO1 is an essential component of biologically induced systemic acquired resistance. Plant Physiology 141, 1666-1675.

Misra, B.B., and Mohapatra, S. (2019). Tools and resources for metabolomics research community: A 2017-2018 update. Electrophoresis 40, 227-246.

Møller, B.L. (1976). Lysine catabolism in barley (Hordeum vulgare L.). Plant Physiology 57, 687-692.

Morrison, R. (1953). The isolation of L-pipecolinic acid from Trifolium repens. Biochemical Journal 53, 474.

Mou, Z., Fan, W., and Dong, X. (2003). Inducers of plant systemic acquired resistance regulate NPR1 function through redox changes. Cell 113, 935-944.

Mur, L.A., Kenton, P., Atzorn, R., Miersch, O., and Wasternack, C. (2006). The outcomes of concentration-specific interactions between salicylate and jasmonate signaling include synergy, antagonism, and oxidative stress leading to cell death. Plant Physiology 140, 249-262.

Mustafa, N.R., Kim, H.K., Choi, Y.H., Erkelens, C., Lefeber, A.W., Spijksma, G., van der Heijden, R., and Verpoorte, R. (2009). Biosynthesis of salicylic acid in fungus elicited Catharanthus roseus cells. Phytochemistry 70, 532-539.

Navarova, H., Bernsdorff, F., Doring, A.C., and Zeier, J. (2012). Pipecolic acid, an endogenous mediator of defense amplification and priming, is a critical regulator of inducible plant immunity. The Plant Cell 24, 5123-5141. 
Nawrath, C. (2002). EDS5, an essential component of salicylic acid-dependent signaling for disease resistance in arabidopsis, is a member of the MATE transporter family. The Plant Cell 14, 275-286.

Nawrath, C., and Métraux, J.-P. (1999). Salicylic acid induction-deficient mutants of Arabidopsis express PR- 2 and PR-5 and accumulate high levels of camalexin after pathogen inoculation. The Plant Cell 11, 1393-1404.

Nobuta, K., Okrent, R.A., Stoutemyer, M., Rodibaugh, N., Kempema, L., Wildermuth, M.C., and Innes, R.W. (2007). The GH3 acyl adenylase family member PBS3 regulates salicylic acid-dependent defense responses in Arabidopsis. Plant Physiology 144, 1144-1156.

Okrent, R.A., Brooks, M.D., and Wildermuth, M.C. (2009). Arabidopsis GH3.12 (PBS3) conjugates amino acids to 4-substituted benzoates and is inhibited by salicylate. Journal of Biological Chemistry 284, 9742-9754.

Park, S.-W., Kaimoyo, E., Kumar, D., Mosher, S., and Klessig, D.F. (2007). Methyl salicylate is a critical mobile signal for plant systemic acquired resistance. Science 318, 113-116.

Patti, G.J., Yanes, O., and Siuzdak, G. (2012). Innovation: Metabolomics: the apogee of the omics trilogy. Nature Reviews Molecular Cell Biology 13, 263-269.

Pelludat, C., Brem, D., and Heesemann, J. (2003). Irp9, encoded by the highpathogenicity island of Yersinia enterocolitica, is able to convert chorismate into salicylate, the precursor of the siderophore Yersiniabactin. Journal of Bacteriology 185, 5648-5653.

Piasecka, A., Jedrzejczak-Rey, N., and Bednarek, P. (2015). Secondary metabolites in plant innate immunity: conserved function of divergent chemicals. New Phytologist 206, 948-964.

Potter, D.W., and Hinson, J.A. (1986). Reactions of $N$-acetyl- $p$-benzoquinone imine with reduced glutathione, acetaminophen, and NADPH. Molecular Pharmacology 30, 33-41.

Ramirez-Carrasco, G., Martinez-Aguilar, K., and Alvarez-Venegas, R. (2017). Transgenerational defense priming for crop protection against plant pathogens: a hypothesis. Frontiers in Plant Science 8, 696.

Raskin, I., Ehmann, A., Melander, W.R., and Meeuse, B.J. (1987). Salicylic acid: a natural inducer of heat production in Arum lilies. Science 237, 1601-1602.

Ravanel, S., Block, M.A., Rippert, P., Jabrin, S., Curien, G., Rébeillé, F., and Douce, R. (2004). Methionine Metabolism in Plants: CHLOROPLASTS ARE AUTONOMOUS FOR DE NOVO METHIONINE SYNTHESIS AND CAN IMPORT S-ADENOSYLMETHIONINE FROM THE CYTOSOL. Journal of Biological Chemistry 279, 22548-22557.

Reimer-Michalski, E.M., and Conrath, U. (2016). Innate immune memory in plants. Seminars in Immunology 28, 319-327.

Riedlmeier, M., Ghirardo, A., Wenig, M., Knappe, C., Koch, K., Georgii, E., Dey, S., Parker, J.E., Schnitzler, J.P., and Vlot, A.C. (2017). Monoterpenes support systemic acquired resistance within and between Plants. The Plant Cell 29, 14401459.

Roberts, L.D., Souza, A.L., Gerszten, R.E., and Clish, C.B. (2012). Targeted metabolomics. Current Protocols in Molecular Biology Chapter 30, Unit 3032 31-24.

Ross, A.F. (1961). Systemic acquired resistance induced by localized virus infections in plants. Virology 14, 340-358.

Rothstein, M., and Miller, L.L. (1954). The conversion of lysine to pipcolic acid in the rat. Journal of Biological Chemistry 211, 851-858. 
Schütte, H., and Seelig, G. (1967). Zur Biosynthese der Pipecolinsäure in Phaseolus vulgaris. Zeitschrift für Naturforschung B 22, 824-826.

Schymanski, E.L., Ruttkies, C., Krauss, M., Brouard, C., Kind, T., Duhrkop, K., Allen, F., Vaniya, A., Verdegem, D., Bocker, S., Rousu, J., Shen, H., Tsugawa, H., Sajed, T., Fiehn, O., Ghesquiere, B., and Neumann, S. (2017). Critical assessment of small molecule identification 2016: automated methods. Journal of Cheminformatics 9, 22.

Serino, L., Reimmann, C., Baur, H., Beyeler, M., Visca, P., and Haas, D. (1995). Structural genes for salicylate biosynthesis from chorismate in Pseudomonas aeruginosa. Molecular and General Genetics 249, 217-228.

Serrano, M., Wang, B., Aryal, B., Garcion, C., Abou-Mansour, E., Heck, S., Geisler, M., Mauch, F., Nawrath, C., and Metraux, J.P. (2013). Export of salicylic acid from the chloroplast requires the multidrug and toxin extrusion-like transporter EDS5. Plant Physiology 162, 1815-1821.

Seybold, H., Trempel, F., Ranf, S., Scheel, D., Romeis, T., and Lee, J. (2014). Ca ${ }^{2+}$ signalling in plant immune response: from pattern recognition receptors to $\mathrm{Ca}^{2+}$ decoding mechanisms. New Phytologist 204, 782-790.

Seyfferth, C., and Tsuda, K. (2014). Salicylic acid signal transduction: the initiation of biosynthesis, perception and transcriptional reprogramming. Frontiers in Plant Science 5, 697.

Shah, J., and Zeier, J. (2013). Long-distance communication and signal amplification in systemic acquired resistance. Frontiers in Plant Science 4, 30.

Sharma, S., Shinde, S., and Verslues, P.E. (2013). Functional characterization of an ornithine cyclodeaminase-like protein of Arabidopsis thaliana. BMC Plant Biology 13, 182.

Sherp, A.M., Westfall, C.S., Alvarez, S., and Jez, J.M. (2018). Arabidopsis thaliana GH3.15 acyl acid amido synthetase has a highly specific substrate preference for the auxin precursor indole-3-butyric acid. Journal of Biological Chemistry 293, 4277-4288.

Shulaev, V., Leon, J., and Raskin, I. (1995). Is salicylic acid a translocated signal of systemic acquired resistance in tobacco? The Plant Cell 7, 1691-1701.

Singh, A., Lim, G.H., and Kachroo, P. (2017). Transport of chemical signals in systemic acquired resistance. Journal of Integrative Plant Biology 59, 336-344.

Smith, C.A., O'Maille, G., Want, E.J., Qin, C., Trauger, S.A., Brandon, T.R., Custodio, D.E., Abagyan, R., and Siuzdak, G. (2005). METLIN: a metabolite mass spectral database. Therapeutic Drug Monitoring 27, 747-751.

Smith, R., Mathis, A.D., Ventura, D., and Prince, J.T. (2014). Proteomics, lipidomics, metabolomics: a mass spectrometry tutorial from a computer scientist's point of view. BMC Bioinformatics 15, S9.

Song, J.T. (2006). Induction of a salicylic acid glucosyltransferase, AtSGT1, is an early disease response in Arabidopsis thaliana. Molecules \& Cells 22.

Song, J.T., Lu, H., and Greenberg, J.T. (2004a). Divergent roles in Arabidopsis thaliana development and defense of two homologous genes, aberrant growth and death 2 and AGD2-like defense response protein 1, encoding novel aminotransferases. The Plant Cell 16, 353-366.

Song, J.T., Lu, H., McDowell, J.M., and Greenberg, J.T. (2004b). A key role for ALD1 in activation of local and systemic defenses in Arabidopsis. The Plant Journal 40, 200212.

Song, J.T., Koo, Y.J., Seo, H.S., Kim, M.C., Choi, Y.D., and Kim, J.H. (2008). Overexpression of AtSGT1, an Arabidopsis salicylic acid glucosyltransferase, leads to increased susceptibility to Pseudomonas syringae. Phytochemistry 69, 1128-1134. 
Spoel, S.H. (2003). NPR1 modulates cross-talk between salicylate- and jasmonatedependent defense pathways through a novel function in the cytosol. The Plant Cell 15, 760-770.

Staswick, P.E. (2002). Jasmonate response locus JAR1 and several related Arabidopsis genes encode enzymes of the firefly luciferase superfamily that show activity on jasmonic, salicylic, and indole-3-acetic acids in an assay for adenylation. The Plant Cell 14, 1405-1415.

Staswick, P.E., and Tiryaki, I. (2004). The oxylipin signal jasmonic acid is activated by an enzyme that conjugates it to isoleucine in Arabidopsis. The Plant Cell 16, 21172127.

Staswick, P.E., Serban, B., Rowe, M., Tiryaki, I., Maldonado, M.T., Maldonado, M.C., and Suza, W. (2005). Characterization of an Arabidopsis enzyme family that conjugates amino acids to indole-3-acetic acid. The Plant Cell 17, 616-627.

Strawn, M.A., Marr, S.K., Inoue, K., Inada, N., Zubieta, C., and Wildermuth, M.C. (2007). Arabidopsis isochorismate synthase functional in pathogen-induced salicylate biosynthesis exhibits properties consistent with a role in diverse stress responses. Journal of Biological Chemistry 282, 5919-5933.

Stringlis, I.A., Yu, K., Feussner, K., de Jonge, R., Van Bentum, S., Van Verk, M.C., Berendsen, R.L., Bakker, P., Feussner, I., and Pieterse, C.M.J. (2018). MYB72dependent coumarin exudation shapes root microbiome assembly to promote plant health. Proceedings of the National Academy of Sciences of the United States of America 115, E5213-E5222.

Tada, Y., Spoel, S.H., Pajerowska-Mukhtar, K., Mou, Z., Song, J., Wang, C., Zuo, J., and Dong, $\mathbf{X}$. (2008). Plant immunity requires conformational charges of NPR1 via Snitrosylation and thioredoxins. Science 321, 952-956.

Tateda, C., Zhang, Z., Shrestha, J., Jelenska, J., Chinchilla, D., and Greenberg, J.T. (2014). Salicylic acid regulates Arabidopsis microbial pattern receptor kinase levels and signaling. The Plant Cell 26, 4171-4187.

The Arabidopsis Genome Initiative. (2000). Analysis of the genome sequence of the flowering plant Arabidopsis thaliana. Nature 408, 796.

Tohge, T., Perez de Souza, L., and Fernie, A.R. (2018). On the natural diversity of phenylacylated-flavonoid and their in planta function under conditions of stress. Phytochemistry Reviews 17, 279-290.

Trennheuser, F., Burkhard, G., and Becker, H. (1994). Anthocerodiazonin an alkaloid from Anthoceros agrestis. Phytochemistry 37, 899-903.

Tripathi, D., Jiang, Y.-L., and Kumar, D. (2010). SABP2, a methyl salicylate esterase is required for the systemic acquired resistance induced by acibenzolar-S-methyl in plants. FEBS Letters 584, 3458-3463.

Truman, W., Bennett, M.H., Kubigsteltig, I., Turnbull, C., and Grant, M. (2007). Arabidopsis systemic immunity uses conserved defense signaling pathways and is mediated by jasmonates. Proceedings of the National Academy of Sciences of the United States of America 104, 1075-1080.

Tsuda, K., and Katagiri, F. (2010). Comparing signaling mechanisms engaged in patterntriggered and effector-triggered immunity. Current Opinion in Plant Biology 13, 459-465.

Tugizimana, F., Mhlongo, M.I., Piater, L.A., and Dubery, I.A. (2018). Metabolomics in plant priming research: the way forward? International Journal of Molecular Sciences 19.

van Loon, L.C. (2016). The intelligent behavior of plants. Trends in Plant Science 21, 286294. 
Vernooij, B., Friedrich, L., Morse, A., Reist, R., Kolditz-Jawhar, R., Ward, E., Uknes, S., Kessmann, H., and Ryals, J. (1994). Salicylic acid is not the translocated signal responsible for inducing systemic acquired resistance but is required in signal transduction. The Plant Cell 6, 959-965.

Vogt, T., and Jones, P. (2000). Glycosyltransferases in plant natural product synthesis: characterization of a supergene family. Trends in Plant Science 5, 380-386.

Walsh, C.T., Liu, J., Rusnak, F., and Sakaitani, M. (1990). Molecular studies on enzymes in chorismate metabolism and the enterobactin biosynthetic pathway. Chemical Reviews 90, 1105-1129.

Wang, C., El-Shetehy, M., Shine, M.B., Yu, K., Navarre, D., Wendehenne, D., Kachroo, A., and Kachroo, P. (2014). Free radicals mediate systemic acquired resistance. Cell Reports 7, 348-355.

Wang, C., Liu, R., Lim, G.-H., de Lorenzo, L., Yu, K., Zhang, K., Hunt, A.G., Kachroo, A., and Kachroo, P. (2018). Pipecolic acid confers systemic immunity by regulating free radicals. Science Advances 4, eaar4509.

Warren, R.F., Merritt, P.M., Holub, E., and Innes, R.W. (1999). Identification of three putative signal transduction genes involved in $R$ gene-specified disease resistance in Arabidopsis. Genetics 152, 401-412.

Wasternack, C., and Strnad, M. (2019). Jasmonates are signals in the biosynthesis of secondary metabolites - Pathways, transcription factors and applied aspects - A brief review. New Biotechnology 48, 1-11.

Wendehenne, D., Gao, Q.M., Kachroo, A., and Kachroo, P. (2014). Free radicalmediated systemic immunity in plants. Current Opinion in Plant Biology 20, 127134.

Weng, J.K. (2014). The evolutionary paths towards complexity: a metabolic perspective. New Phytologist 201, 1141-1149.

Westfall, C.S., Zubieta, C., Herrmann, J., Kapp, U., Nanao, M.H., and Jez, J.M. (2012). Structural basis for prereceptor modulation of plant hormones by GH3 proteins. Science 336, 1708-1711.

Weyrich, L.S., Duchene, S., Soubrier, J., Arriola, L., Llamas, B., Breen, J., Morris, A.G., Alt, K.W., Caramelli, D., Dresely, V., Farrell, M., Farrer, A.G., Francken, M., Gully, N., Haak, W., Hardy, K., Harvati, K., Held, P., Holmes, E.C., Kaidonis, J., Lalueza-Fox, C., de la Rasilla, M., Rosas, A., Semal, P., Soltysiak, A., Townsend, G., Usai, D., Wahl, J., Huson, D.H., Dobney, K., and Cooper, A. (2017). Neanderthal behaviour, diet, and disease inferred from ancient DNA in dental calculus. Nature 544, 357-361.

Widhalm, J.R., Gutensohn, M., Yoo, H., Adebesin, F., Qian, Y., Guo, L., Jaini, R., Lynch, J.H., McCoy, R.M., Shreve, J.T., Thimmapuram, J., Rhodes, D., Morgan, J.A., and Dudareva, N. (2015). Identification of a plastidial phenylalanine exporter that influences flux distribution through the phenylalanine biosynthetic network. Nature Communications 6, 8142.

Wiermer, M., Feys, B.J., and Parker, J.E. (2005). Plant immunity: the EDS1 regulatory node. Current Opinion in Plant Biology 8, 383-389.

Wildermuth, M.C. (2006). Variations on a theme: synthesis and modification of plant benzoic acids. Current Opinion in Plant Biology 9, 288-296.

Wildermuth, M.C., Dewdney, J., Wu, G., and Ausubel, F.M. (2001). Isochorismate synthase is required to synthesize salicylic acid for plant defence. Nature 414, 562 .

Winter, D., Vinegar, B., Nahal, H., Ammar, R., Wilson, G.V., and Provart, N.J. (2007). An "Electronic Fluorescent Pictograph" browser for exploring and analyzing largescale biological data sets. PloS One 2, e718. 
Wu, Y., Zhang, D., Chu, J.Y., Boyle, P., Wang, Y., Brindle, I.D., De Luca, V., and Despres, C. (2012). The Arabidopsis NPR1 protein is a receptor for the plant defense hormone salicylic acid. Cell Reports 1, 639-647.

Xu, B., Fan, Z., Lei, Y., Ping, Y., Jaisi, A., and Xiao, Y. (2018). Insights into pipecolic acid biosynthesis in Huperzia serrata. Organic Letters 20, 2195-2198.

Yalpani, N., Enyedi, A.J., León, J., and Raskin, I. (1994). Ultraviolet light and ozone stimulate accumulation of salicylic acid, pathogenesis-related proteins and virus resistance in tobacco. Planta 193, 372-376.

Yan, C., and Xie, D. (2015). Jasmonate in plant defence: sentinel or double agent? Plant Biotechnology Journal 13, 1233-1240.

Yi, H.S., Heil, M., Adame-Alvarez, R.M., Ballhorn, D.J., and Ryu, C.M. (2009). Airborne induction and priming of plant defenses against a bacterial pathogen. Plant Physiology 151, 2152-2161.

Yokoo, S., Inoue, S., Suzuki, N., Amakawa, N., Matsui, H., Nakagami, H., Takahashi, A., Arai, R., and Katou, S. (2018). Comparative analysis of plant isochorismate synthases reveals structural mechanisms underlying their distinct biochemical properties. Bioscience Reports 38.

Yu, D., Qanmber, G., Lu, L., Wang, L., Li, J., Yang, Z., Liu, Z., Li, Y., Chen, Q., Mendu, V., Li, F., and Yang, Z. (2018). Genome-wide analysis of cotton GH3 subfamily II reveals functional divergence in fiber development, hormone response and plant architecture. BMC Plant Biology 18, 350.

Yu, K., Soares, J.M., Mandal, M.K., Wang, C., Chanda, B., Gifford, A.N., Fowler, J.S., Navarre, D., Kachroo, A., and Kachroo, P. (2013). A feedback regulatory loop between G3P and lipid transfer proteins DIR1 and AZI1 mediates azelaic-acidinduced systemic immunity. Cell Reports 3, 1266-1278.

Zacharius, R., Thompson, J., and Steward, F. (1952). The detection, isolation and identification of (-)-pipecolic acid as a constituent of plants. Journal of the American Chemical Society 74, 2949-2949.

Zeier, J. (2013). New insights into the regulation of plant immunity by amino acid metabolic pathways. Plant, Cell \& Environment 36, 2085-2103.

Zhang, M., and Robertus, J.D. (2002). Molecular cloning and characterization of a fulllength flavin-dependent monooxygenase from yeast. Archives of Biochemistry and Biophysics 403, 277-283.

Zhang, Y., Yang, Y., Fang, B., Gannon, P., Ding, P., Li, X., and Zhang, Y. (2010a). Arabidopsis snc2-1D activates receptor-like protein-mediated immunity transduced through WRKY70. The Plant Cell 22, 3153-3163.

Zhang, Y., Xu, S., Ding, P., Wang, D., Cheng, Y.T., He, J., Gao, M., Xu, F., Li, Y., and Zhu, Z. (2010b). Control of salicylic acid synthesis and systemic acquired resistance by two members of a plant-specific family of transcription factors. Proceedings of the National Academy of Sciences of the United States of America 107, 18220-18225.

Zhang, Z., Li, Q., Li, Z., Staswick, P.E., Wang, M., Zhu, Y., and He, Z. (2007). Dual regulation role of GH3.5 in salicylic acid and auxin signaling during ArabidopsisPseudomonas syringae interaction. Plant Physiology 145, 450-464.

Zhao, H., Chen, D., Li, Y., and Cai, B. (2005). Overexpression, purification and characterization of a new salicylate hydroxylase from naphthalene-degrading Pseudomonas sp. strain ND6. Microbiological Research 160, 307-313.

Zhao, Y., Luo, L., Xu, J., Xin, P., Guo, H., Wu, J., Bai, L., Wang, G., Chu, J., Zuo, J., Yu, H., Huang, $\mathbf{X}$., and Li, J. (2018). Malate transported from chloroplast to mitochondrion triggers production of ROS and PCD in Arabidopsis thaliana. Cell Research 28, 448-461. 
Zheng, Z., Qualley, A., Fan, B., Dudareva, N., and Chen, Z. (2009). An important role of a BAHD acyl transferase-like protein in plant innate immunity. The Plant Journal 57, 1040-1053.

Zhou, Y., Memelink, J., and Linthorst, H.J.M. (2018). An E. coli biosensor for screening of cDNA libraries for isochorismate pyruvate lyase-encoding cDNAs. Molecular Genetics and Genomics 293, 1181-1190.

Zipfel, C., Kunze, G., Chinchilla, D., Caniard, A., Jones, J.D., Boller, T., and Felix, G. (2006). Perception of the bacterial PAMP EF-Tu by the receptor EFR restricts Agrobacterium-mediated transformation. Cell 125, 749-760. 


\section{Supplementary materials}

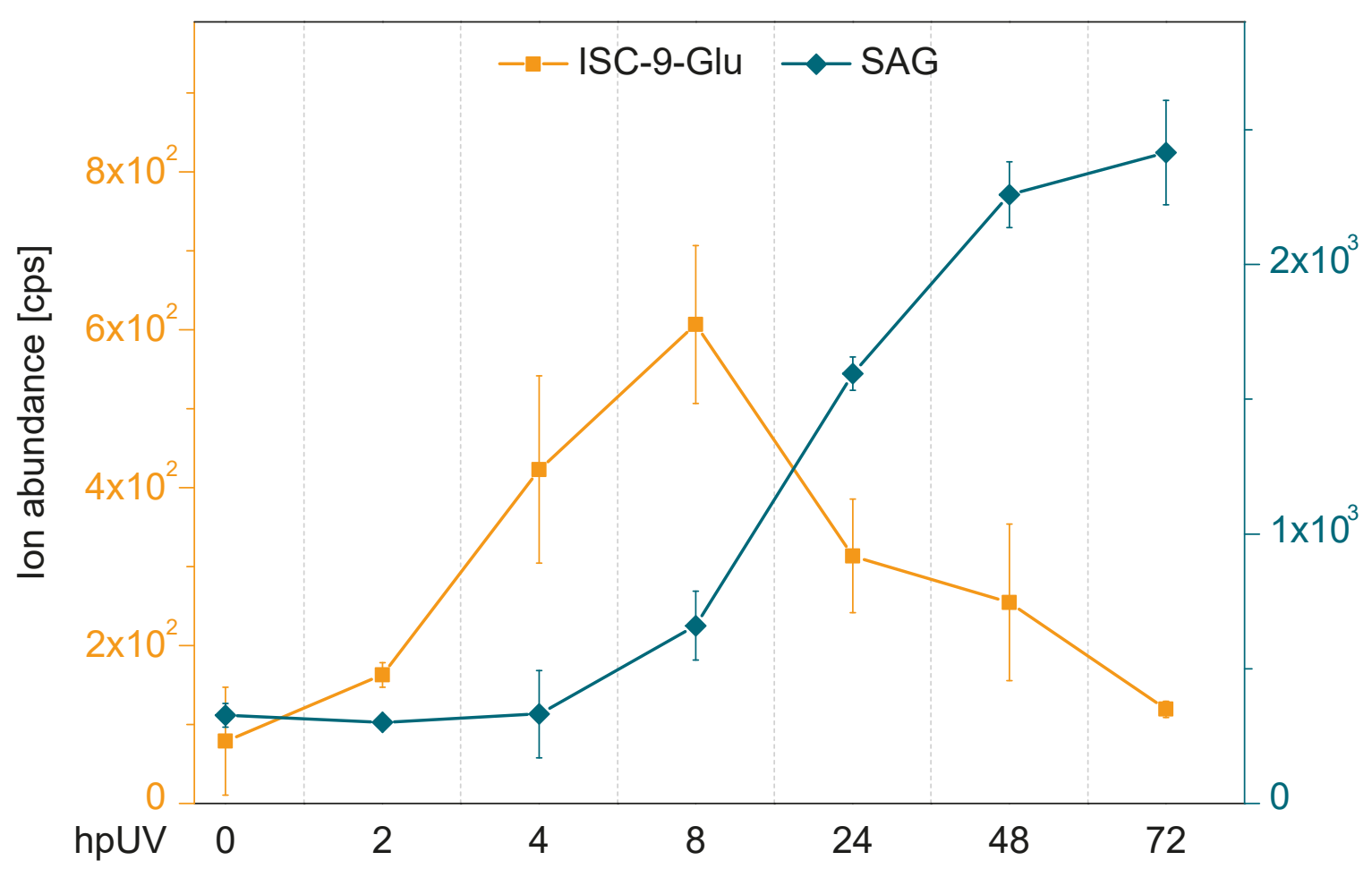

Supplementary Figure 1. UV-C treatment of Arabidopsis thaliana leads to an accumulation of the SA precursor isochorismate-9-glutamate and SA glycoside.

Levels of isochorismate-9-glutamate (ISC-9-Glu, orange graph) and SA glycoside (SAG, blue graph). The ion abundance of ISC-9-Glu and SAG in Arabidopsis wild type leaves (Col-0) at different time points after UV-C light treatment is depicted in counts per second [cps]. Data points represent the mean \pm STD of three biological replicates. Statistical differences among replicates are labeled with different letters $(\mathrm{P}<0.05$, one-way ANOVA and post hoc Tukey's Test; $\mathrm{n}=3$ ). 

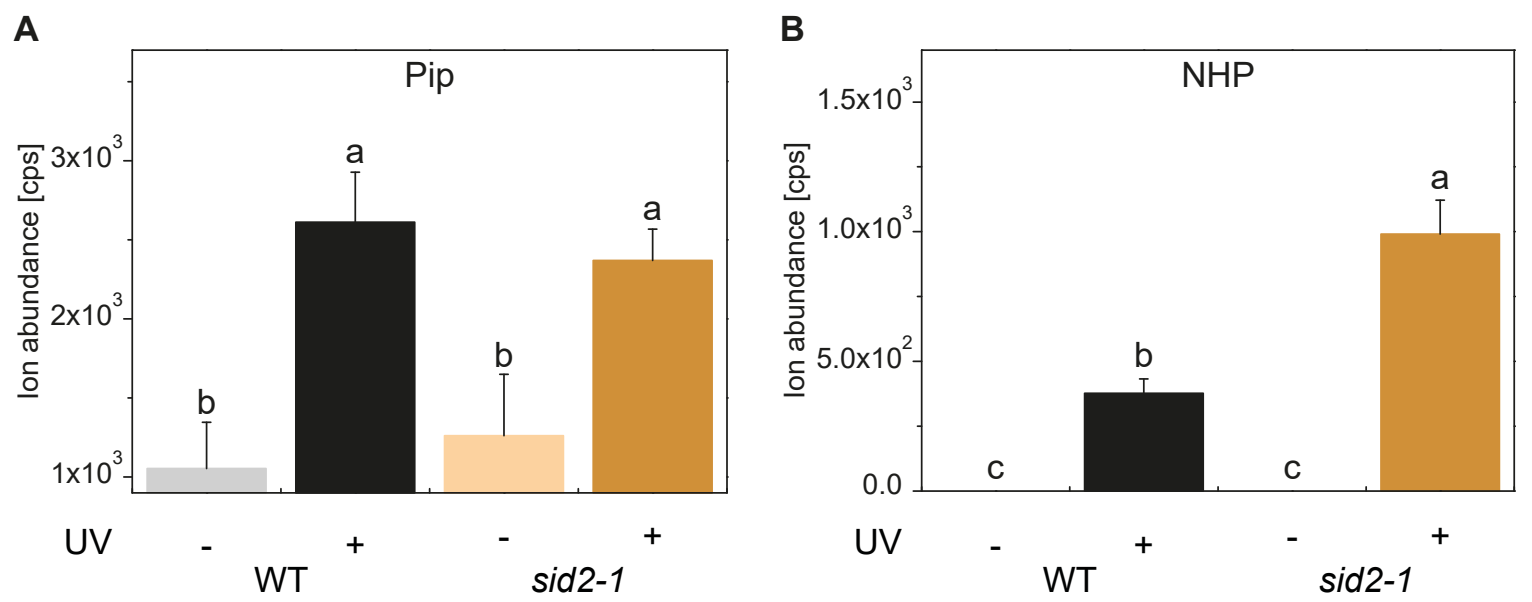

Supplementary Figure 2. UV-C induced NHP biosynthesis is not disturbed in sid 2 mutant plants. Upon UV-C treatment, wild type as well as SA deficient sid2 mutant plants produce substantial amounts of (A) pipecolic acid (Pip) and (B) N-hydroxy pipecolic acid (NHP). As reported previously, sid2 mutants are not affected in their Pip/NHP biosynthesis (Hartmann et al., 2018). On the contrary, significantly more NHP can be measured in the sid2 mutant than in the wild type plants. Bars represent the mean \pm STD of three biological replicates. Statistical differences among replicates are labeled with different letters $(\mathrm{P}<0.05$, one-way ANOVA and post hoc Tukey's Test; $\mathrm{n}=3$ ) 


\section{Acknowledgment}

First of all, I would like to thank Prof. Ivo Feussner, for convincing me to take over this great project, for motivating and challenging me throughout the time I was working on it, and for the organization of PRoTECT. Many thanks to Prof. Christiane Gatz and Dr. Corinna Thurow for all the fruitful discussions and the great feedback I got from you not only during the TCM meetings. Your doors were always open for me. Thank you so much Prof. Yuelin Zhang for all the sincere hospitality during my time in Vancouver. Your open mind and great support encouraged large parts of the results I could achieve.

I cannot stress enough how grateful I am to Dr. Kirstin Feussner and Dr. Sven Haroth. You have been so patient with me and my ideas, you taught me so many things. It is easy to say for me that without your support, I would never have been able to do what I have done and to become the researcher that I am. Thank you so much.

I would like to acknowledge all members of the IRTG PRoTECT in Goettingen and Vancouver. Especially Dr. Yuli Ding who become a close friend during the time in Canada. Thanks to my wonderful flat mates in Vancouver Milena and Sina, who made the time there very entertaining. Many thanks to all the IRTG members for the great time we had, for the scientific meetings, hiking trips, kayak tours etc. I very much enjoyed to spend the time with you.

Thanks a lot to all the wonderful people in the Plant Biochemistry Department. All the little things, from small talk in the queue for coffee, over sharing soil and media, to our Christmas movie nights, I very much enjoyed to be part of this team. It was always great to have supportive people around that are in a similar situation.

I would like to thank my rugby mates, who made the time in Goettingen just awesome. It was such a pleasure to leave the work behind and join a training session or a game. I could get my head free and meet some very special people there.

Next, I want to thank my parents. I do not think that there is anybody else in the world, who was more interested in the research I did than you. You were always curious about the plants, bugs, genes and so on I was currently occupied with. Thanks so much for all your patient and advices. The most important person at the end, thank you so much Ann-Kathrin. No one is as open minded, supportive and warm-hearted as you. To have you by my side helped me to go through the most challenging situations. 


\section{CURRICULUM VITAE}

\section{PERSONAL Information}

Dmitrij Rekhter

Date of birth: $\quad 24.05 .1990$

Place of birth: $\quad$ Charkow, Ukraine

Nationality: German

\section{Personal Information}

since $04 / 2016$

Göttingen Graduate Center of Neurosciences, Biophysics, and Molecular Biosciences (GGNB), University of Goettingen

DFG Fellow in the Department of Plant Biochemistry, within the International Research Training Group 2172 "Plant Responses To Eliminate Critical Threats"

Thesis title: Metabolic Signals in Systemic Acquired Resistance

10/2013-03/2016 Master of Science Program Microbiology and Biochemistry, University of Goettingen

Department of Plant Biochemistry, University of Goettingen Thesis title: Metabolic Signals in Systemic Acquired Resistance

10/2010-09/2013 Bachelor of Science Program Human and Molecular Biology, Saarland University

Department of Biophysics, Saarland University

Thesis title: Characterization of hydroxylated Coenzyme Q10:

Redox properties in cell system

08/1997-06/2010 General qualification for university entrance

Kaiser-Wilhelm- und Ratsgymnasium, Hannover, Germany 


\section{Publications:}

Ding, P. *, Rekhter, D. *, Ding, Y. *, Feussner, K., Busta, L., Haroth, S., Xu, S., Li, X., Jetter, R., Feussner, I. and Zhang, Y. (2016). Characterization of a pipecolic acid biosynthesis pathway required for systemic acquired resistance. The Plant Cell, 28, 2603-2615. doi: 10.1105/tpc.16.00486.

Eng, F., Haroth, S., Feussner, K., Meldau, D., Rekhter, D., Ischebeck, T., Brodhun, F. and Feussner, I. (2016). Optimized jasmonic acid production by Lasiodiplodia theobromae reveals formation of valuable plant secondary metabolites. PLoS ONE, 11, e0167627. doi: 10.1371/journal.pone.0167627.

Pinter, N., Hach, C.A., Hampel, M., Rekhter, D., Zienkiewicz, K., Feussner, I., Poehlein, A., Daniel, R., Finkernagel, F., and Heimel, K. (2019). Signal peptide peptidase activity connects the unfolded protein response to plant defense suppression by Ustilago maydis. PLoS Pathogens 15, e1007734. doi: 10.1371/journal.ppat.1007734.

*Contributed equally to this study and should be considered as co-first authors. 